\title{
Functional characterization and molecular identification of neuroprotective receptors for erythropoietin-like ligands
}

\author{
Dissertation \\ for the award of the degree \\ "Doctor rerum naturalium" \\ of the Georg-August-University Goettingen \\ within the doctoral program Biology \\ of the Georg-August University School of Science (GAUSS) \\ submitted by \\ Nina Hahn \\ from Goettingen
}

Goettingen, 2019 


\section{Thesis committee}

Prof. Dr. Ralf Heinrich

Department of Cellular Neurobiology

Georg-August-University Goettingen

Prof. Dr. Gregor Bucher

Department of Developmental Biology

Georg-August-University Goettingen

\section{Further members of the examination board}

PD Dr. Sven Bradler

Department of Animal Evolution and Biodiversity

Georg-August-University Göttingen

Dr. Nico Posnien

Department of Developmental Biology

Georg-August-University Goettingen

Prof. Dr. Manuela Schmidt

Somatosensory Signaling Group

Max-Planck-Institute for Experimental Medicine Goettingen

PD Dr. Hauke Werner

Department of Neurogenetics

Max-Planck-Institute for Experimental Medicine Goettingen

Date of the oral examination: $13^{\text {th }}$ December, 2019 


\section{Declaration}

I hereby declare that the doctoral thesis entitled "Functional characterization and molecular identification of neuroprotective receptors for erythropoietin-like ligands" has been written independently and with no other sources and aids than quoted.

Date

Signature

Nina Hahn 
"Two roads diverged in a wood, and II took the one less traveled by, And that has made all the difference."

- Robert Frost 


\section{SUMMARY}

The cytokine erythropoietin (Epo) regulates erythropoiesis but also protects mammalian neurons from apoptosis. However, receptors and transduction pathways involved in neuroprotection are only partially characterized. Although insects lack Epo and classical Epo receptor genes, human recombinant Epo induces protection and promotes neuroregeneration of insect neurons. Thus, insect neurons are a suitable model system to study neuroprotective signaling pathways independent from erythropoietic pathways. My studies on insect primary brain neurons demonstrated that recombinant human Epo protects neurons against hypoxia-induced apoptosis by activating orthologs of cytokine receptor like factor 3 (CRLF3), a predicted cytokine receptor with unknown ligand and function in mammals. To this end, I established soaking RNA interference to knock down orthologs of CRLF3 in primary brain cell cultures of the beetle Tribolium castaneum and the locust Locusta migratoria.

Furthermore, I tested various Epo-like molecules which have been described in vertebrates to be neuroprotective but not erythropoietic. All tested Epo-like molecules were also neuroprotective in locust primary brain neurons. These shared neuroprotective functions propose that the ligandbinding structures of neuroprotective receptors in insects and vertebrates are more similar than those of the neuroprotective and erythropoietic receptors within vertebrates. Moreover, I provided evidence that neuroprotective effects of the human splice variant EV-3 and an Epo-mimetic peptide are mediated via CRLF3.

Phylogenetic analysis of 293 CRLF3 sequences revealed that CRLF3 emerged with the origin of the eumetazoan nervous system. This coincidence suggests that CRLF3 might have originally evolved for a function within the nervous system. However, I have demonstrated CRLF3 expression also in other tissues apart from the nervous system in human cell lines, Tribolium castaneum and Locusta migratoria. In both human neuroblastoma (SH-SY5Y) cells and Tribolium tissue CRLF3 expression is regulated by hypoxic stress. Moreover, I showed that CRLF3 expression is present at all developmental stages of Tribolium but to a variable extent.

In this study, I tested the hypothesis that CRLF3 might be a neuroprotective Epo receptor. The results presented herein show that CRLF3 is crucial to mediate the neuroprotective effect of human Epo and Epo-like cell protective molecules in insect primary brain cells. Whether this function of CRLF3 is also present in human brain cells has to be proven in future experiments for which I have already established pivotal tools. This study introducing CRLF3 as a neuroprotective Epo receptor might support the development of safe, neuroprotective therapies that selectively stimulate CRLF3. 


\section{TABLE OF CONTENTS}

1

2

3

4

4.1 AIM AND STRUCTURE OF THE THESIS.

RESULTS

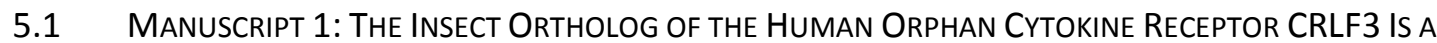
NeUROPRotective ERYTHROPOIETIN RECEPTOR

5.2 Manuscript 2: The ORPhan Cytokine ReCEPTOR CRLF3 EMERged With THe ORIGIN OF THE Nervous System AND IS A NeURoprotective ERYthropoietin RECEPTOR IN LOCUSTS

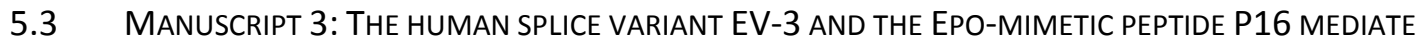
NEUROPROTECTION VIA THE ORPHAN CRLF3 RECEPTOR IN LOCUSTS

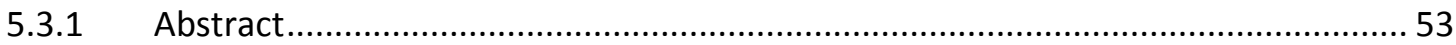

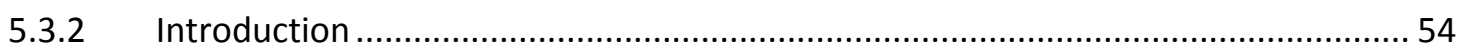

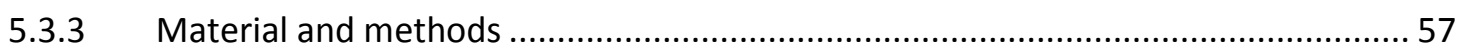

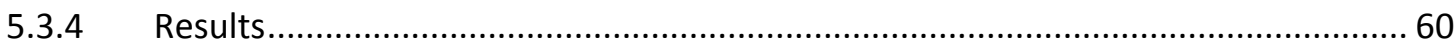

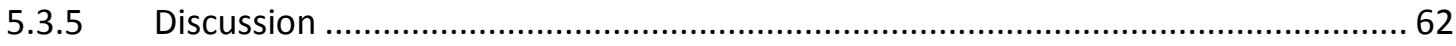

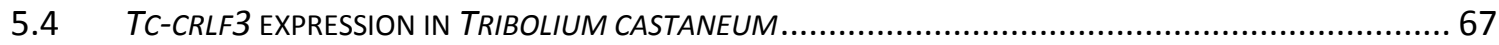

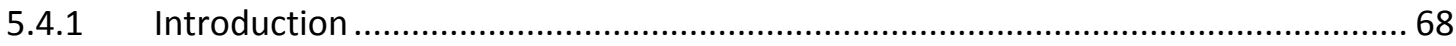

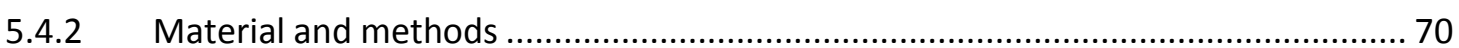

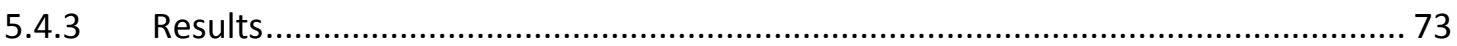

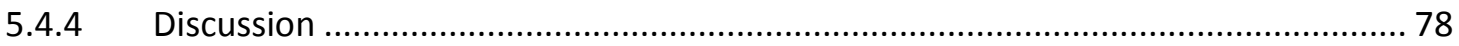

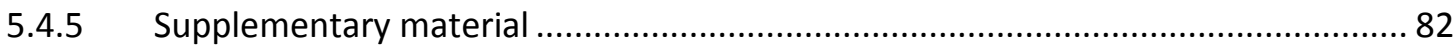

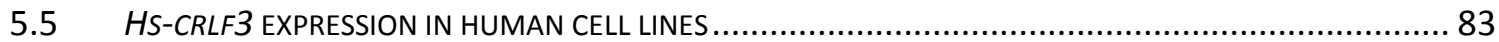

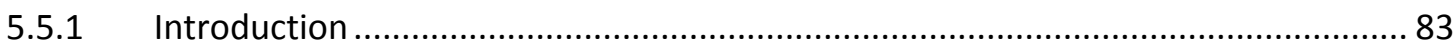

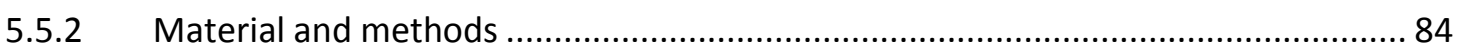

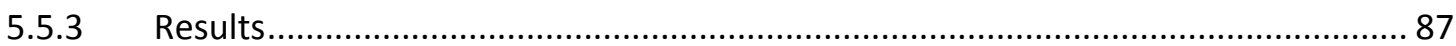




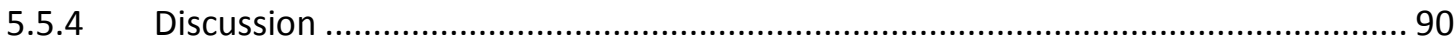

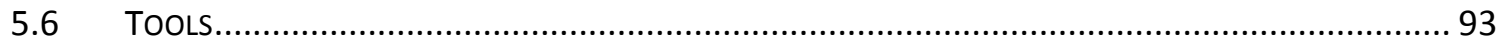

5.6.1 Cell viability assay with HEK293 cells....................................................... 94

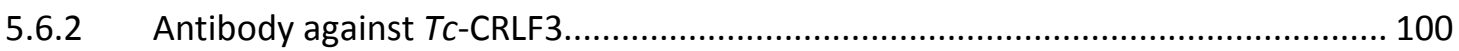

5.6.3 Drosophila S2 cells heterologously expressing Tc-CRLF3 ............................... 103

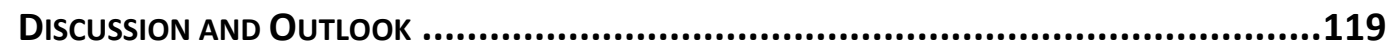

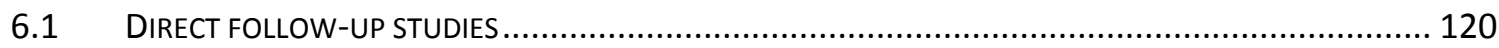

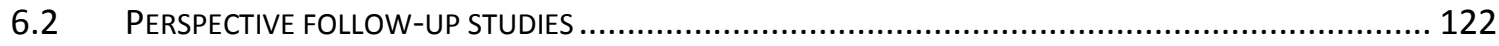

6.2.1 Investigating the downstream signaling pathways of Tc-CRLF3 using RNA

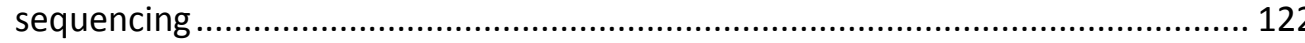

6.2.2 Determination of TC-CRLF3 signaling and binding motifs............................... 124

6.2.3 Potential approaches to find the endogenous ligand of CRLF3....................... 125

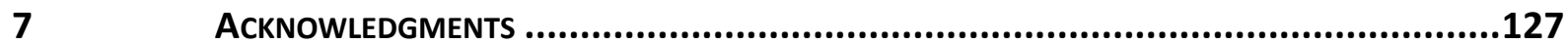

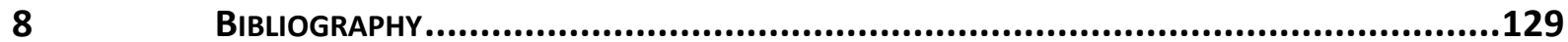

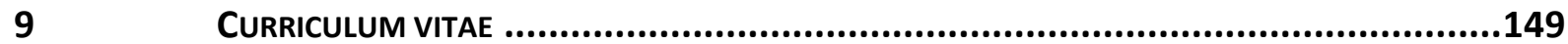




\section{LIST OF ABBREVIATIONS}

A

aa

BLAST $^{\circledast} \quad$ Basic Local Alignment Search Tool

bp base pairs

C Cytosine

CHBP Cyclized Helix B Peptide

CRLF3 Cytokine Receptor-Like Factor 3

$\mathbf{C}_{\mathrm{T}} \quad$ Cycle Threshold

DABCO 1,4-diazobicyclo[2.2.2]octane

DAPI 4',6-diamidino-2-phenylindole, dihydrochloride

DEG Differentially expressed gene

DIG Digoxigenin

DMSO Dimethyl Sulfoxide

DNA Deoxyribonucleic Acid

EDTA Ethylenediaminetetraacetic Acid

eGFP enhanced Green Fluorescent Protein

EphB4 Ephrin B4

Epo Erythropoietin

EpoR Erythropoietin Receptor

EpoR/BcR EpoR/beta-common heteroreceptor

EpoR $_{\mathbf{2}}$ homodimeric Erythropoietin Receptor

eYFP enhanced Yellow Fluorescent Protein 
Fetal Bovine Serum

$\mathbf{G}$

Guanine

GhR Growth hormone Receptor

GO

Gene Ontology

HEK293 Human Embryonic Kidney 293

ISH In Situ Hybridization

JAK Janus Kinase

kb Kilobase (1000 bp)

kD Kilodalton

KEGG Kyoto Encyclopedia of Genes and Genomes

PBS Phosphate Buffered Saline

PBT Phosphate Buffered saline + Tween $_{20}$

PCR Polymerase Chain Reaction

PI Propidium lodide

PI3K/AKT Phosphoinositide-3-Kinase /AKT

PrIR Prolactin Receptor

pRNAi parental RNA interference

qRT-PCR quantitative Real-Time Polymerase Chain Reaction

RA Retinoic Acid

Ras/MAPK Ras/Mitogen-Activated Protein Kinase

RNA seq Ribonucleic Acid sequencing

RNA Ribonucleic Acid

RNAi RNA interference

RT-PCR Reverse Transcription Polymerase Chain Reaction 
S

Synthesis

SDS-PAGE Sodium Dodecyl Sulfate Polyacrylamide Gel Electrophoresis

shRNA short hairpin RNA

SNP Single Nucleotide Polymorphism

STAT Signal Transducers and Activators of Transcription

T Thymine

TpoR Thrombopoietin Receptor

TSA Tyramide Signal Amplification

$\alpha$

anti

$\mathbf{Y}_{\mathrm{c}} \quad$ common gamma chain 


\section{LIST OF FIGURES}

Figure 4-1: Comparison of Epo signaling in erythropoiesis and neuroprotection.

Figure 4-2: Presence of Epo's neuroprotective effect, Epo, EpoR and CRLF3 amongst mammals and insects.

Figure 5-1: Graphic representation of the Epo sequence and corresponding Epo-like molecules....... 56

Figure 5-2: DAPI nuclear staining for live/dead cell evaluation. ....................................................... 58

Figure 5-3: Impact of Epo and Epo-mimetic peptides on locust neuronal survival in hypoxia............. 60

Figure 5-4: Effects of EV-3 and P16 on neuronal survival after Lm-CRLF3 knock-down..................... 61

Figure 5-5: Evaluation of Tc-crlf3 expression after parental RNAi injecting pupae. ............................ 73

Figure 5-6: $24-48 \mathrm{~h}$ old whole-body embryos stained by a NBT/BCIP color reaction of the TCcrlf3 and eyfp (detecting egfp) probes for $19 \mathrm{~h}$ at $4^{\circ} \mathrm{C}$.

Figure 5-7: Fluorescent in situ staining (FISH) in Tribolium whole mount larval brains........................ 75

Figure 5-8: Expression levels of $T c-c r l f 3$ in different developmental stages. .75

Figure 5-9: Tc-crlf3 expression levels after $36 \mathrm{~h}$ hypoxia exposure in different developmental stages.

Figure 5-10: Tc-crlf3 expression levels in the brain after $36 \mathrm{~h}$ exposure to hypoxia.

Figure 5-11: Hs-crlf3 expression in various human cell lines.

Figure 5-12: Hs-crlf3 expression in differentiated (RA) and non-differentiated (wt)

neuroblastoma SH-SY5Y cells upon $24 \mathrm{~h}$ hypoxia exposure.

Figure 5-13: Hs-crlf3 expression in HEK293 cells treated with camptothecin. 89

Figure 5-14: Hs-crlf3 expression in HEK293 cells treated with Epo and etoposide. 89

Figure 5-15: Morphological changes of HEK293 cells after induction of apoptosis using different concentrations of etoposide $(10 \mu \mathrm{M}$ to $30 \mu \mathrm{M})$. 96

Figure 5-16: Cell viability assessment of HEK293 cells using TUNEL assay and cell cycle analysis. 98

Figure 5-17: Design of the immunogenic peptide of the custom-made antibody targeting TcCRLF3 (425 aa). 100

Figure 5-18: Western blot testing the specificity of the custom-made antibody against Tc-CRLF3 from rabbit No. 2.

Figure 5-19: Western blot analysis to evaluate the transfection efficiency in Drosophila S2 cells. ... 105

Figure 5-20: Mass spectrometric analysis of the fusion protein Tc-CRLF3-eGFP heterologously expressed in Drosophila S2 cells.

Figure 5-21: Localization of eGFP in transfected Drosophila S2 cells. 108

Figure 5-22: Flow cytometric evaluation of Drosophila S2 cells heterologously expressing TcCRLF3-eGFP and cell viability. 
Figure 5-23: Cell cycle analysis of staurosporine- and starvation-challenged S2 cells. 110 


\section{LIST OF TABLES}

Table 5-1: Sequences of Epo-mimetic peptides and control peptides. 57

Table 5-2: Summary of previously investigated Epo-like molecules and their functions. 63

Table 5-3: Summary of primers for qRT-PCR analysis and cloning of Tribolium genes... 70

Table 5-4: Summary of primers that were used for qRT-PCR analysis of human cell lines. 84

Table 5-5: Culture media for human cell lines. .85

Table 6-1: Draft of experimental conditions for RNA seq including all potential control groups....... 123 


\section{INTRODUCTION}

The prevalence of neurodegenerative diseases is rising worldwide. Given that the risk of being affected increases with age, extending life expectancies will further contribute to growing numbers of affected people. Alzheimer's disease and Parkinson's disease are the most common neurodegenerative diseases but stroke is the second leading cause of mortality all over the world (Murray and Lopez, 1997; WHO, 2006). Stroke is defined by sudden interruption of blood flow in the brain and can be subdivided into hemorrhagic and ischemic strokes. Hemorrhagic strokes are caused by the rupture of a blood vessel while ischemic strokes are caused by clogged blood vessels. In most countries, more than $80 \%$ of strokes are ischemic (WHO, 2006). The interruption of the blood supply leads to a local lack of oxygen in the brain immediately damaging neurons and other brain cells. Hence, a rapid diagnosis and therapy is inevitable to prevent loss of neurons and functional impairments (Heiss and Rosner, 1983; Marler et al., 2000). As a rule of thumb, the three-hourwindow predicts that treatment within the first three hours after stroke onset significantly increases the recovery rate (Hacke et al., 2004; Powers et al., 2018). Even though major advances in the diagnosis of stroke (e.g. perfusion computer tomography (CT), CT angiography, magnetic resonance angiography) have been made, safe therapies are still missing or in their early beginnings (WHO, 2006). The most common therapy is the administration of aspirin to restore blood circulation and to resupply the ischemic area with oxygen (Powers et al., 2018). However, loss of neurons cannot be fully prevented with this therapy. To conclude, neurodegenerative diseases including Alzheimer's as well as Parkinson's diseases and stroke are amongst the most frequent global burdens to human health but safe therapies are still pending. Hence, there is an urgent need to investigate and develop new neuroprotective therapies.

The cytokine erythropoietin (Epo) protects neurons from apoptosis. It has been successfully used to protect neurons damaged by stroke, Parkinson's disease or Alzheimer's disease (Ehrenreich et al., 2002; Kadota et al., 2009; Lee et al., 2012; Pedroso et al., 2018). Epo was initially discovered as a positive regulator of erythropoiesis in vertebrates produced by the kidney or liver (reviewed in (Jelkmann, 1992)). Besides this hormonal function, paracrine Epo signaling has been detected in various tissues including the nervous system suggesting a more general cell protective effect (Arcasoy, 2010; Chateauvieux et al., 2011; Ogunshola and Bogdanova, 2013; Zhang et al., 2014b). While its signaling pathway in erythropoiesis is well studied, the cell protective anti-apoptotic signaling pathways are diverse and only partially characterized (Figure 4-1). In the erythropoietic system, physiological stress (e.g. hypoxia) initiates Epo binding to the classical homodimeric Epo receptor (EpoR) on erythroid precursor cells preventing their apoptosis (Klingmüller et al., 1996; 
Matthews et al., 1996). This results in an increase of red blood cells enhancing the capability to bind atmospheric oxygen.

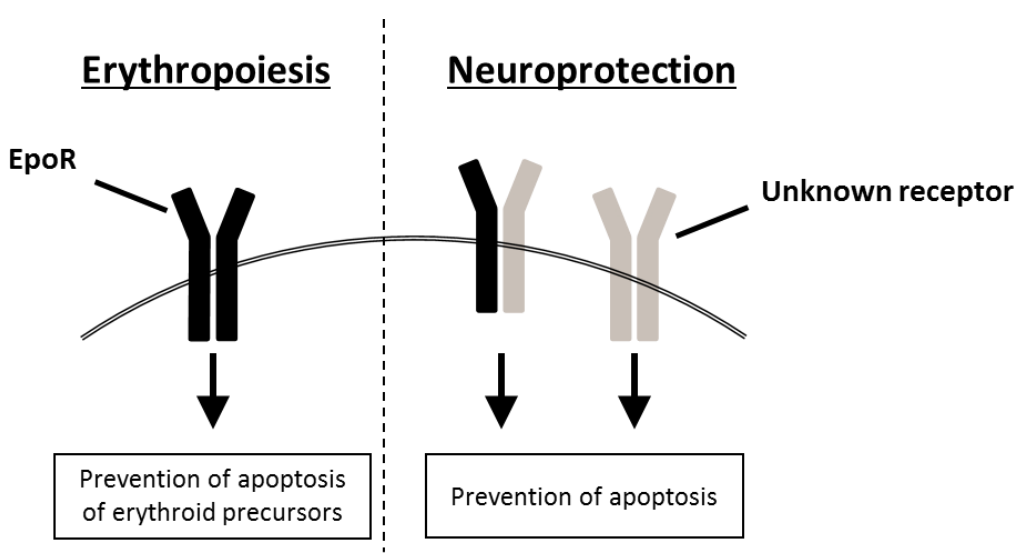

Figure 4-1: Comparison of Epo signaling in erythropoiesis and neuroprotection. To induce erythropoiesis (left), Epo binds to the classical homodimeric EpoR $\mathrm{R}_{2}$ receptor on erythroid precursor cells preventing apoptosis. In turn, this results in an increase of red blood cells. Epo-mediated neuroprotection signaling (right) is still enigmatic. Epo may bind to homodimeric or heterodimeric receptors with or without EpoR inducing intracellular anti-apoptotic pathways.

In general cell protective systems, Epo presumably binds to homo- or heterodimeric receptors with or without contribution of the classical EpoR inducing intracellular anti-apoptotic pathways. Epo has already been tested in a phase II/III clinical trial focusing on stroke treatment. However, the outcome was negative due to adverse side effects that may arose from interference with the erythropoietic pathway (Ehrenreich et al., 2009; Souvenir et al., 2015). The discovery of Epo variants and Epo-like molecules being neuroprotective but not erythropoietic suggested the presence of neuroprotective Epo receptors different from the classical EpoR (Bonnas et al., 2017; Brines et al., 2008; Leist et al., 2004; Livnah et al., 1996). Potential alternative neuroprotective Epo receptors are the heterodimeric EpoR/B-common receptor complex and ephrin B4 receptor (EphB4) in vertebrates (Brines et al., 2004; Ostrowski and Heinrich, 2018; Pradeep et al., 2015).

Here, I introduce the cytokine receptor-like factor 3 (CRLF3) as another potential neuroprotective Epo-receptor. Like the classical EpoR, CRLF3 is a member of prototypic class I group 1 cytokine receptors (Liongue and Ward, 2007). Since its ligand is still unknown, it is termed as an orphan cytokine receptor. I have initially selected this receptor since its presence matched the presence of Epo's neuroprotective effect in different species (Figure 4-2). Previous studies have demonstrated that recombinant human Epo protects locust neurons against hypoxia-induced neuronal apoptosis and promotes neuroregeneration. These findings were particularly intriguing, since insects lack epo and epoR genes (Gocht et al., 2009; Miljus et al., 2014; Ostrowski et al., 2011). They suggest that an Epo-like cell protective signaling pathway might have been present in the urbilaterian ancestor of 
insects and mammals (Ostrowski et al., 2011) (Ostrowski 2011). However, Epo-induced neuroprotective effects were absent in Drosophila (Heinrich et al., 2017).

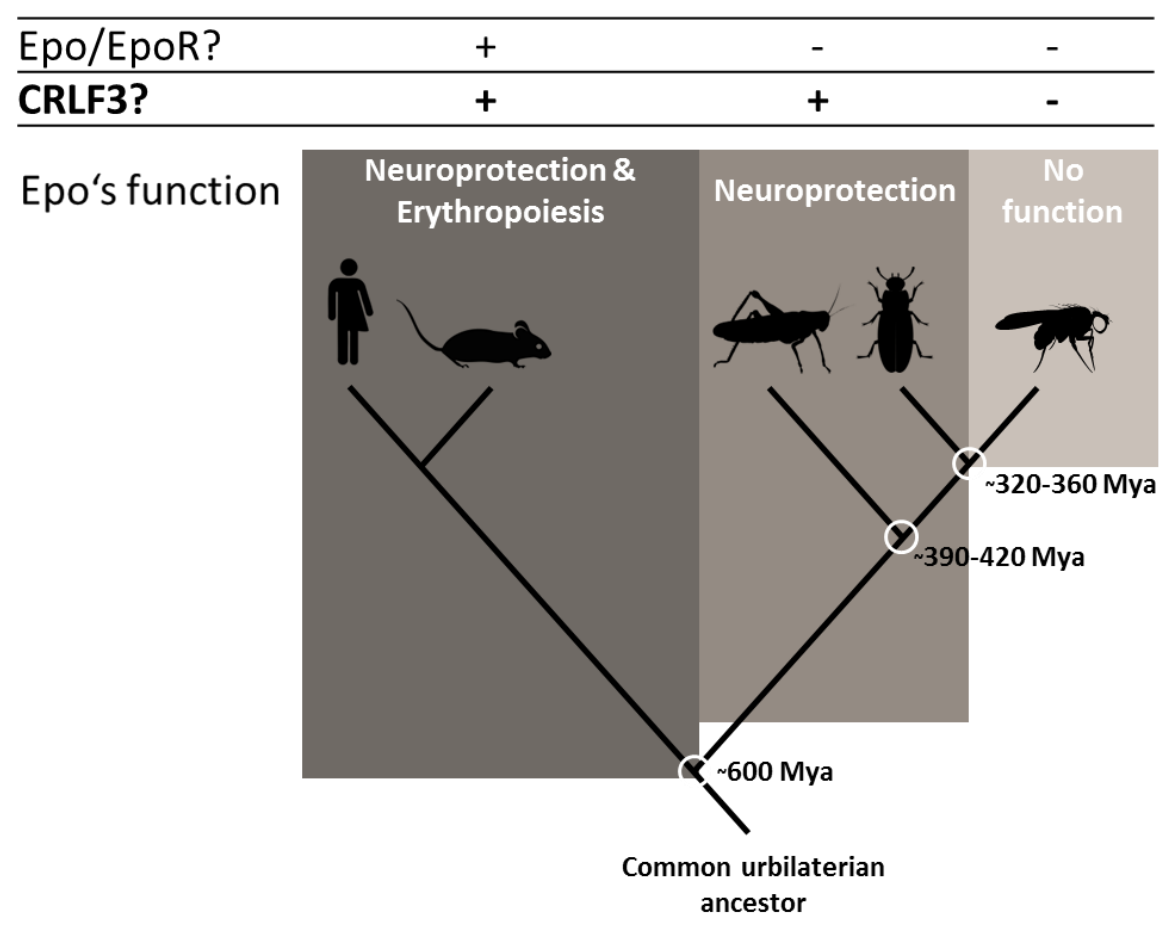

Figure 4-2: Presence of Epo's neuroprotective effect, Epo, EpoR and CRLF3 amongst mammals and insects. Epo induces both, neuroprotection and erythropoiesis, in mammals. In addition, Epo induces neuroprotection in the locust Locusta migratoria and the beetle Tribolium castaneum although insects lack orthologs of Epo and EpoR. In contrast, there is no effect on Drosophila cells in which CRLF3 orthologs are, in comparison the other species, missing. Modified after Heinrich et al., 2017.

In addition, it was known that Tribolium expresses CRLF3 but not Drosophila (Wyder et al., 2007). Hence, I tested whether Tribolium brain cells are sensitive to Epo. I could show that Tribolium brain cells are indeed Epo sensitive and that the presence of the CRLF3 ortholog was critical for Epoinduced neuroprotection (Hahn et al., 2017). Later, I identified a crlf3 ortholog in locusts which further encouraged me to study CRLF3 as a putative neuroprotective Epo receptor (Hahn et al., 2019). Using insect models to study this research question is advantageous in two ways. The beetle genome is well-annotated and many genetic tools are readily available but more importantly it allows studying the neuroprotective signaling pathways in the absence of erythropoietic signaling. 


\subsection{Aim and structure of the thesis}

My thesis aims to characterize the orphan cytokine receptor CRLF3 and to test its potential involvement in Epo-mediated neuroprotection. I started to investigate insect orthologs of CRLF3 and later broadened the scope to human Hs-CRLF3. The results chapter comprises three first author manuscripts and three further subchapters that present additional results and newly established tools for follow-up studies. The structure is as follows:

- Manuscript 1, Hahn et al. 2017, introduces the beetle ortholog TC-CRLF3 as a neuroprotective Epo receptor. Furthermore, a convenient method for loss-of-function studies in Tribolium primary brain cells, termed soaking RNA interference, was established.

- Manuscript 2, Hahn et al. 2019, focuses on the locust ortholog Lm-CRLF3. Using RACE PCR, I identified $L m$-crlf3 and demonstrated that $L m$-CRLF3 is crucial for Epo-induced neuroprotection. I detected CRLF3 expression in other tissues besides the nervous system and presented that soaking RNA interference is also applicable to locust primary brain cells. Furthermore, I provided a phylogenetic analysis of CRLF3 sequences revealing the simultaneous emergence of CRLF3 and the eumetazoan nervous system.

- Manuscript 3 is in preparation and investigates neuroprotective properties of Epo-mimetic peptides in locust primary brain cells, which are selectively neuroprotective in humans without stimulating erythropoiesis. All tested Epo-mimetic peptides induced neuroprotection in locust brain cells suggesting a large structural similarity of neuroprotective Epo receptors in insect and mammalian brains. The effects of P16 and the human splice variant EV-3 are mediated by $L m$-CRLF3.

- A subchapter investigating Tc-CRLF3 expression in Tribolium using in situ hybridization to localize $c r l f 3$ transcripts and quantitative real-time PCR to determine $T c$-crlf3 expression levels. I detected Tc-crlf3 expression in Tribolium embryos and in the larval brain. Moreover, quantitative analysis revealed that expression levels vary among developmental stages and following exposure to hypoxic stress.

- A subchapter dealing with Hs-crlf3 expression in human cell lines. Hs-crlf3 was detected in various cell lines including human embryonal kidney (HEK293) cells and neuroblastoma (SHSY5Y) cells. While differentiation of neuroblastoma SH-SY5Y cells increased $\mathrm{Hs}$-crlf3 expression, it is reduced by $24 \mathrm{~h}$ hypoxia exposure. Furthermore, I examined the response of $H s$-crlf3 expression to Epo and the chemical stressor etoposide.

- A subchapter presenting tools that I have established and that are ready to use for follow-up studies. I generated a custom-made antibody targeting TC-CRLF3 to study protein localization as well as protein levels. Furthermore, I introduced two different methods to determine the cell viability of HEK293 cells as well as two methods for the evaluation of S2 cell viability using a flow cytometer. Finally, I describe the implementation of a new assay comprising Drosophila S2 cells that heterologously express the beetle receptor Tc-CRLF3. 


\section{RESULTS}

\subsection{Manuscript 1: The Insect Ortholog of the Human Orphan Cytokine Receptor CRLF3 Is a Neuroprotective Erythropoietin Receptor}

The manuscript "The Insect Ortholog of the Human Orphan Cytokine Receptor CRLF3 Is a Neuroprotective Erythropoietin Receptor" was published in "Frontiers in Molecular Neuroscience" on $14^{\text {th }}$ July 2017.

Authors: Nina Hahn, Debbra Y. Knorr, Johannes Liebig, Liane Wüstefeld, Karsten Peters, Marita Büscher, Gregor Bucher, Hannelore Ehrenreich and Ralf Heinrich.

This manuscript presents the first insect orthologs of the human orphan cytokine receptor CRLF3. It characterized the beetle ortholog Tc-CRLF3 as a neuroprotective Epo receptor in Tribolium primary brain neurons providing the first evidence that CRLF3 might be of interest for Epo-mediated neuroprotective therapies. Firstly, we established primary brain cell cultures of Tribolium castaneum. We then showed that Tribolium primary brain neurons are protected against hypoxia-induced apoptosis by human recombinant Epo although insects do not endogenously express Epo. However, knock-down of Tc-CRLF3 expression abolished Epo's neuroprotective effect. For these loss of function studies, we established a new RNA interference (RNAi) method for Tribolium brain cells in vitro, called soaking RNAi. Soaking RNAi is the supplementation of culture medium with $10 \mathrm{ng} / \mu \mathrm{l}$ double-stranded RNA for a couple of days. It is a very convenient approach to knock down a protein of interest because cells spontaneously take up double-stranded RNA without requiring transfection procedures.

My contribution to this paper was substantial. I collected data together with DYK, JL and KP, analyzed data and prepared figures. Furthermore, I supervised JL. The study was designed together with GB, $\mathrm{MB}, \mathrm{LW}, \mathrm{HE}$ and $\mathrm{RH}$. RH and I wrote the manuscript and I accompanied the review process. 


\section{OPEN ACCESS}

Edited by:

Karl Tsim,

Hong Kong University of Science and Technology, Hong Kong

Reviewed by:

Michele Papa,

Università degli Studi della Campania

"Luigi Vanvitelli" Caserta, Italy

Laura Maria Frago,

Universidad Autonoma de Madrid,

Spain

*Correspondence: Ralf Heinrich rheinri1@gwdg.de

Received: 20 December 2016 Accepted: 28 June 2017 Published: 14 July 2017

Citation: Hahn N, Knorr DY, Liebig J, Wüstefeld L, Peters K, Büscher M,

Bucher G, Ehrenreich $\mathrm{H}$ and Heinrich R (2017) The Insect Ortholog of the Human Orphan Cytokine Receptor CRLF3 Is a Neuroprotective Erythropoietin Receptor

Front. Mol. Neurosci. 10:223. doi: 10.3389/fnmol.2017.00223

\section{The Insect Ortholog of the Human Orphan Cytokine Receptor CRLF3 Is a Neuroprotective Erythropoietin Receptor}

\author{
Nina Hahn ${ }^{1}$, Debbra Y. Knorr ${ }^{1}$, Johannes Liebig ${ }^{1}$, Liane Wüstefeld ${ }^{2,3}$, Karsten Peters ${ }^{1}$, \\ Marita Büscher ${ }^{4}$, Gregor Bucher ${ }^{4}$, Hannelore Ehrenreich ${ }^{2,3}$ and Ralf Heinrich ${ }^{1 *}$ \\ ${ }^{1}$ Department of Cellular Neurobiology, Institute for Zoology, Georg-August-University Goettingen, Goettingen, Germany, \\ ${ }^{2}$ Clinical Neuroscience, Max Planck Institute of Experimental Medicine, Goettingen, Germany, ${ }^{3}$ DFG Center for Nanoscale \\ Microscopy and Molecular Physiology of the Brain (CNMPB), Goettingen, Germany, ${ }^{4}$ Department of Evolutionary \\ Developmental Biology, Institute for Zoology, Georg-August-University Goettingen, Goettingen, Germany
}

The cytokine erythropoietin (Epo) mediates various cell homeostatic responses to environmental challenges and pathological insults. While stimulation of vertebrate erythrocyte production is mediated by homodimeric "classical" Epo receptors, alternative receptors are involved in neuroprotection. However, their identity remains enigmatic due to complex cytokine ligand and receptor interactions and conflicting experimental results. Besides the classical Epo receptor, the family of type I cytokine receptors also includes the poorly characterized orphan cytokine receptor-like factor 3 (CRLF3) present in vertebrates including human and various insect species. By making use of the more simple genetic makeup of insect model systems, we studied whether CRLF3 is a neuroprotective Epo receptor in animals. We identified a single ortholog of CRLF3 in the beetle Tribolium castaneum, and established protocols for primary neuronal cell cultures from Tribolium brains and efficient in vitro RNA interference. Recombinant human Epo as well as the non-erythropoietic Epo splice variant EV-3 increased the survival of serum-deprived brain neurons, confirming the previously described neuroprotective effect of Epo in insects. Moreover, Epo completely prevented hypoxia-induced apoptotic cell death of primary neuronal cultures. Knockdown of CRLF3 expression by RNA interference with two different double stranded RNA (dsRNA) fragments abolished the neuroprotective effect of Epo, indicating that CRLF3 is a crucial component of the insect Epo-responsive receptor. This suggests that a common urbilaterian ancestor of the orphan human and insect cytokine receptor CRLF3 served as a neuroprotective receptor for an Epo-like cytokine. Our work also suggests that vertebrate CRLF3, like its insect ortholog, might represent a tissue protection-mediating receptor.

Keywords: erythropoietin, non-erythropoietic splice variant, neuroprotection, cytokine receptor, primary neuronal cell culture, RNA interference, Tribolium castaneum

\section{INTRODUCTION}

Erythropoietin (Epo) is a positive regulator of vertebrate erythropoiesis. Circulating hormonal Epo, predominantly released by the kidney in adult mammals, stimulates homodimeric Epo receptors ("classical EpoR") on Epo-responsive erythroid progenitor cells in the bone marrow that initiate anti-apoptotic mechanisms leading to increased numbers of red blood cells (Jelkmann, 1992). 
Epo is also synthesized and released as a para- or autocrine signal in various tissues that initiates adaptive cellular responses to injury and physiological challenges in order to support cell survival, maintenance of functions and regeneration (Noguchi et al., 2008; Sargin et al., 2010; Chateauvieux et al., 2011). In the nervous system, Epo is produced by glia, neurons and endothelial cells. Epo plays important roles during mammalian brain development (Alnaeeli et al., 2012), acts as a protectant that secures neuronal survival and functionality under ischemic, cytotoxic and otherwise deleterious conditions (Brines and Cerami, 2005; Arcasoy, 2008; Sargin et al., 2010), supports regeneration (Kretz et al., 2005; King et al., 2007) and enhances cognitive performance in healthy and diseased brains (Sargin et al., 2011; Kästner et al., 2012). Although expression of classical EpoR in the brain has been demonstrated by numerous studies, Epo receptors mediating neuroprotection seem to be different from the classical homodimeric EpoR involved in erythropoiesis (Leist et al., 2004; Brines and Cerami, 2005). Several Epo-like molecules have been demonstrated to lack erythropoietic functions while retaining full neuroprotective activity compared to normal Epo. Such tissuespecific agonists include the derivatives carbamylated Epo and asialoerythropoietin (Erbayraktar et al., 2003; Leist et al., 2004), mutant Epo with serine to leucine mutation at position 104 (Gan et al., 2012) and the human Epo splice variant EV-3 with deleted third exon (Bonnas, 2012). The nature of the neuroprotective Epo receptor is currently debated. As non-erythropoietic candidate receptors, common $\beta$ chain receptor/classical EpoR heteromers expressed in the nervous system (Brines and Cerami, 2005; Collino et al., 2015) and bone tissue (Duedal Rölfing et al., 2014) and leukemia inhibitory factor LIFR/DC130 expressed in neuronal and other cell lines (Bonnas, 2012) are discussed. However, even within a particular tissue such as the nervous system, the expression of Epo receptors may depend on cell type, developmental stage, physiological condition and actual as well as previous exposure to challenging stimuli (Sinor and Greenberg, 2000; Brines and Cerami, 2005; Shein et al., 2005; Um et al., 2007; Sanchez et al., 2009). Cells may flexibly respond to different physiological challenges by expressing different Epo receptors that activate particular adaptive cellular responses.

Genes for Epo and classical EpoR are present in all major vertebrate groups including mammals, amphibia and fish (Ostrowski et al., 2011), suggesting that Epo/EpoR signaling already evolved when the vertebrate lineage emerged. Based on protein sequence similarity, which is already quite low between mammals and fish, no orthologs of Epo and EpoR have so far been identified in invertebrate species. Nevertheless, Epo-mediated neuroprotection and neuroregeneration have been described in grasshoppers and locusts (Ostrowski et al., 2011; Miljus et al., 2014). In these studies recombinant human Epo rescued primary cultured brain neurons from hypoxiainduced apoptotic cell death by activating Janus kinase/signal transducers and activators of transcription (JAK/STAT) transduction pathways (Miljus et al., 2014). In addition, locust brain neurons were also protected by the non-erythropoietic human splice variant EV-3 (Miljus et al., 2017; characterization of EV-3 by Bonnas et al., 2017), suggesting that insect and mammalian neuroprotective Epo receptors share common structures that allow activation by both Epo and EV-3. These findings support the hypothesis of a pre-vertebrate evolution of Epo-like signaling in neuroprotection (Brines and Cerami, 2005).

Epo belongs to the group of type I helical cytokines that, in spite of low primary sequence similarity, share spatial similarity emerging from a characteristic four-helix bundle structure (Boulay et al., 2003). Their corresponding receptors (type I cytokine receptors) contain a conserved 200 amino acid "cytokine receptor homology domain" required for ligand binding and some conserved intracellular motifs that allow the activation of JAK/STAT transduction pathways (Boulay et al., 2003; Liongue and Ward, 2007). Cytokine type I receptors typically associate to homo- or heteromeric receptor complexes that may bind several cytokine ligands.

Classical EpoR belongs to group 1 of cytokine type I receptors, which also includes thrombopoietin receptor, prolactin receptor, growth hormone receptor and the orphan cytokine receptor-like factor 3 (CRLF3) of unknown function. CRLF3 (synonyms: CYTOR4, CREME9, p48.2, p48.6 (sequences may deviate by few amino acids at the $5^{\prime}$ end) has been shown to activate STAT3, cause cell cycle arrest in a human embryonic kidney cell line (Yang et al., 2009) and may regulate neuronal morphology and synaptic vesicle formation (Hashimoto et al., 2012). Crlf3 is located on human chromosome 17 and is expressed in most normal human tissues including the nervous system. Variations of $c r l f 3$ sequences have been associated with amyotrophic lateral sclerosis (ALS; Cirulli et al., 2015) and increased CRLF3 content has been detected in various tumor cell lines and freshly isolated tumors (Dang et al., 2006; Yang et al., 2009). Crlf3 is well conserved which allowed the identification of orthologs in various mammals, fish, primitive chordates and insects, such as the beetle Tribolium castaneum and the cricket Gryllus bimaculatus but not in the fly Drosophila melanogaster (Wyder et al., 2007). Interestingly, we were able to show a neuroprotective effect of Epo on T. castaneum (this study) and G. bimaculatus neural cells but failed to do so in D. melanogaster (unpublished results) which led us to hypothesize that CRLF3 might be mediating this effect.

The red flour beetle $T$. castaneum has a fully sequenced genome and is amenable to double stranded RNA (dsRNA)mediated systemic RNAi (Bucher et al., 2002). In order to explore the possibility that beetle CRLF3 ( $T c$-CRLF3) plays a role in Epo-mediated neuroprotection, we established primary neuronal cell cultures from $T$. castaneum brains and effective soaking RNA interference by dsRNA added to the culture medium. We demonstrate that knockdown of Tc-CRLF3 expression abolishes the protective effect of Epo on hypoxia-exposed T. castaneum brain neurons.

\section{MATERIALS AND METHODS}

\section{Plasmids}

A $427 \mathrm{bp}$ long fragment of the red fluorescent protein (dsRed) of the mushroom anemona Discosoma spec. was cloned into pCRII 
vector by the Bucher Lab. Additionally, $300 \mathrm{bp}$ of Rpt 3 were cloned into pJet 1.2 (Thermo Fisher Scientific, Waltham, MA, USA; Ulrich et al., 2015). For RNAi experiments targeting Tc-CRLF3, primer pairs for two different fragments targeting au3.g2971.t1 (Tc000209) were designed (Table 1). The fragment 1 covers $638 \mathrm{bp}$ whereas the fragment 2 is smaller and consists of 527 bp (sequences shown in Supplementary Figure S1). The fragments were cloned into the pCRII vector using the TA Cloning ${ }^{\circledR}$ Kit (Invitrogen, Life Technologies, Darmstadt, Germany). Subsequently, the plasmids were transformed into DH5 $\alpha$ cells and finally purified with the NucleoSpin ${ }^{\circledR}$ Plasmid Kit (Macherey-Nagel, Düren, Germany).

\section{Preparation of dsRNA}

Template DNA was prepared by PCR using M13F and M13R primers with a T7 RNA polymerase promoter sequence attached to the latter (Table 1). In vitro transcription of dsRNA was performed with the MEGAscript ${ }^{\circledR}$ T7 Transcription Kit following the user manual (Life Technologies, Darmstadt, Germany). The resulting RNA pellet was resuspended in injection buffer $\left(1.4 \mathrm{mM} \mathrm{NaCl}, 0.07 \mathrm{mM} \mathrm{Na}_{2} \mathrm{HPO}_{4}, 0.03 \mathrm{mM}\right.$ $\mathrm{KH}_{2} \mathrm{PO}_{4}, 4 \mathrm{mM} \mathrm{KCl}$ ). In order to anneal both strands of the RNA, the mix was incubated at $94^{\circ} \mathrm{C}$ for $5 \mathrm{~min}$ and cooled down slowly in a heatblock. Before use in cell culture experiments it was sterile filtered (Millex ${ }^{\circledR}$. HV, $0.45 \mu \mathrm{m}$, hydrophilic durapore, Millipore, Darmstadt, Germany).

\section{Sequence Comparison}

Information about Tribolium gene sequence and structure was gained from http://bioinf.uni-greifswald.de/gb2/gbrowse/ tribolium4/ working with the augustus3 prediction. Sequences were identified with Basic Local Alignment Search Tool (BLAST; Altschul et al., 1990) using the matrix BLOSUM62 with standard settings and the mode tblastn. Sequence information of other species was retrieved with BLAST at NCBI data bases (Altschul et al., 1990).

Geneious ${ }^{\circledR}$ (Biomatter Ltd., Auckland, New Zealand) was utilized for alignments using the algorithm ClustalW with standard settings. To identify orthologs of Tribolium castaneum CRLF3, a phylogenetic tree was built with the bootstrap resembling method and standard settings as follows: The initial protein sequence alignment was created with the first three hits of Tribolium castaneum and Drosophila melanogaster and the first four hits of Mus musculus. Domains with high quality of alignments were extracted and concatenated for tree building.

Phylogenetic analysis of the CRLF3 protein was also performed with Geneious ${ }^{\circledR}$. Orthologous amino acid sequences were aligned with ClustalW algorithm with standard settings. The tree was calculated using the Geneious ${ }^{\circledR}$ tree builder with Neighbor-Joining method and bootstrap resampling with default settings. The analysis includes CRLF3 orthologs of the human CRLF3 protein identified by NCBI BLAST. They belong to Homo sapiens (GenBank accession number NP_057070.3), Mus musculus (NP_061246.1), Danio rerio (NP_001017817.2), Xenopus laevis (NP_001080600.1), Branchiostoma floridae
(XP_002607148.1), Ciona intestinalis (NP_001107600.1) and Tribolium castaneum (XP_008190449.1).

\section{Animals}

Studies were performed with red flour beetles Tribolium castaneum (wild-type strain San Bernadino). The beetles were bred in plastic boxes filled with a mixture of full grain flour (Wheat flour type 405; Rosenmühle GmbH, Landshut, Germany) and yeast at $27^{\circ} \mathrm{C}$ and $40 \%$ air humidity. The study was conducted exclusively with insects. Studies on insects do not require special permit. All experiments comply with the German laws for animal welfare ("Deutsches Tierschutzgesetz").

\section{Primary Cultures of T. Castaneum Brain Cells}

Primary cultures were established from brains of late pupae. Brains were extracted from head capsules and collected in $2 \mathrm{ml}$ sterile growth medium (Leibovitz's L-15 (Gibco, Karlsruhe, Germany) supplemented with $0.5 \%$ gentamicin (BioReagent, Munich, Germany)). All brains used in one experiment (20 brains per treatment group) were pooled at this stage. After three exchanges of the medium, the brains were exposed to a mixture of collagenase and dispase (each $2 \mathrm{mg} / \mathrm{ml}$; SigmaAldrich, Munich, Germany) for $45 \mathrm{~min}$ at $27^{\circ} \mathrm{C}$ to digest extracellular matrix proteins. Enzymatic activity was terminated by several washes in Hanks' Balanced Salt Solution (Gibco, Karlsruhe, Germany). Brain tissue was passed several times through the tip of a $100 \mu \mathrm{l}$ pipette to dissociate the brain cells. The suspension was centrifuged with $3000 \mathrm{~g}$ for $1 \mathrm{~min}$ and the pellet containing the cells was resuspended in L-15 medium with $0.5 \%$ gentamicin ( $100 \mu \mathrm{l}$ per treatment group). The dissociated cells were equally allocated onto concanavalin A-coated (Sigma-Aldrich, Munich, Germany) round cover slips (Type DKR0; Hartenstein; Würzburg, Germany; $\varnothing 10 \mathrm{~mm}$; $100 \mu \mathrm{l}$ per cover slip) that were placed in sterile plastic culture dishes (Corning Inc., New York, NY, USA; $\varnothing 35 \mathrm{~mm}$ ). After $2 \mathrm{~h}$ the culture dishes were filled to a volume of $2 \mathrm{ml}$ with $\mathrm{L}$ $15 / 0.5 \%$ gentamicin \pm supplements as specified below. Cultures were maintained in an incubator $\left(27^{\circ} \mathrm{C}\right.$; humidified atmosphere) and medium was replaced every 2 days.

\section{Immunocytochemistry}

Primary cell cultures were fixed with $4 \%$ paraformaldehyde dissolved in $0.1 \mathrm{M}$ phosphate buffer for $15 \mathrm{~min}$ at room temperature. Cells were washed with phosphate buffered saline (PBS) and PBS with 0.1\% Triton-X-100 (SigmaAldrich, Munich, Germany; PBST), each three times for $5 \mathrm{~min}$. Cells were incubated for $1 \mathrm{~h}$ with blocking solution containing 2\% normal goat serum (GE Healthcare, Munich, Germany) and $0.25 \%$ bovine serum albumin (MP Biochemicals, Heidelberg, Germany) dissolved in PBST. Primary antisera were incubated over night at $4^{\circ} \mathrm{C}$. To label neuronal membranes anti-horseradish peroxidase (anti-HRP from rabbit, Sigma Aldrich) was applied in 1:500 dilution in blocking buffer. To label the activated form of caspase-3, anti-cleaved caspase-3 serum from rabbit (Calbiochem, Merck, UK) was applied in 1:300 dilution in blocking buffer. After several washes in PBS, 
cells were incubated with 1:200 diluted Alexa 488-coupled anti-rabbit secondary antibody (developed in goat, Molecular Probes, Thermo Fisher Scientific, Waltham, MA, USA) for $2 \mathrm{~h}$ at room temperature. In some experiments, DAPI (Sigma-Aldrich, Munich, Germany; 1:1000) was applied with the secondary antibody. After washing away excess antibody, cover slips with labeled cells were mounted with 1,4-diazobicyclo[2.2.2] octane (DABCO, Roth, Karlsruhe, Germany) on microscopic slides. Edges of cover slips were sealed with transparent nail polish and stainings were analyzed by fluorescence microscopy.

\section{Protective Effect of rhEpo and EV-3 on T. Castaneum Brain Cells In Vitro}

Four-day-old primary cultures were maintained in L-15 medium supplemented with $0.5 \%$ gentamicin. Particular cultures were incubated with different concentrations $(0.32 \mathrm{ng} / \mathrm{ml}, 0.8 \mathrm{ng} / \mathrm{ml}$, $3.2 \mathrm{ng} / \mathrm{ml}, 8 \mathrm{ng} / \mathrm{ml}, 32 \mathrm{ng} / \mathrm{ml}$ ) of recombinant human Epo (rhEpo, NeoRecormon, Roche, Welwyn Garden City, UK) or $(0.42 \mathrm{ng} / \mathrm{ml}, 0.84 \mathrm{ng} / \mathrm{ml})$ of the human non-erythropoietic Epo splice variant EV-3 (IBA GmbH, Göttingen, Germany) for another 3 days. Cell cultures were fixed in paraformaldehyde (4\%, $30 \mathrm{~min}$ ), labeled with DAPI (Sigma-Aldrich, Munich, Germany; 1:1000) and mounted with DABCO (Roth, Karlsruhe, Germany) on microscopic slides to quantify cellular survival (see below).

\section{Soaking RNA Interference}

Primary cultures of $T$. castaneum brains were maintained in L-15 medium supplemented with $0.5 \%$ gentamicin for 4 days. Each experiment, using the cultured cells from the same population of 80 brains, contained four different treatment groups, one control, incubated without any supplement throughout the entire period and three cultures that were supplemented with $10 \mathrm{ng} / \mu \mathrm{l}$ dsRNA to suppress the expression of dsRed (a protein not naturally expressed by the cells), Tc-CRLF3 (T. castaneum ortholog of CRLF3) and (Regulatory particle triple-A ATPase $3(\mathrm{Rpt} 3)$, a protein of the proteasome that is essential for cellular survival), respectively (for dsRNA sequences see Supplementary Figure S1). After 4 days in vitro, cell cultures were fixed and labeled with DAPI as described above.

\section{Survival in Hypoxia}

Primary cultures of $T$. castaneum brains were maintained in L-15 medium supplemented with $0.5 \%$ gentamicin for 5 days. Each experiment, using the cultured cells from the same population of 100 brains, contained five different treatment groups: (1) untreated control maintained in normoxic condition; (2) untreated control exposed to hypoxia; (3) rhEpotreated culture exposed to hypoxia; (4) rhEpo-treated culture exposed to hypoxia after RNAi-mediated suppression of TcCRLF3 expression; and (5) hypoxia-exposed culture after RNAi mediated suppression of Tc-CRLF3 expression. dsRNA (final concentration $10 \mathrm{ng} / \mu \mathrm{l}$ ) was present in the respective cultures throughout the entire experimental period. To minimize the risk for off-target effects, two dsRNA constructs (see Supplementary
Figure S1) that target non-overlapping sequences were used to interfere with Tc-CRLF3 expression. $\operatorname{rhEpo}(0.8 \mathrm{ng} / \mathrm{ml})$ was added to the respective cultures $12 \mathrm{~h}$ before the hypoxic period. Hypoxia was generated by floating an airproof chamber $(22.5 \times 4.5 \times 4 \mathrm{~cm}$, workshop of our institute) with nitrogen $\left(\mathrm{N}_{2}\right)$. Hypoxia $\left(\mathrm{O}_{2}\right.$ level $\left.\leq 2 \%\right)$ was monitored with an oxygen analyzer (Greisinger GOX 100, Conrad Electronics, Hirschau, Germany) and maintained for $36 \mathrm{~h}$. Afterwards, cultures were reoxygenated, maintained for $12 \mathrm{~h}$ in normoxia, and subsequently fixed and labeled as described above.

\section{Data Analysis}

Assessment of cellular survival was based on chromatin structure revealed by fluorescent labeling with DAPI (Gocht et al., 2009). Using a fluorescence microscope (Zeiss Axioskop, Oberkochen, Germany) with $63 \times$ oil objective, equipped with a CCD camera (Spot RT3, Visitron, Puchheim, Germany), two continuous rows of non-overlapping photographs were taken (typically $\sim 120$ per culture), that extended over the entire cover slip to the right and the left of the center. Numbers of nuclei from alive and dead cells (at the time of fixation) were manually counted with the freeware software Fiji and its cell counter plugin (ImageJ by NIH). Relative numbers of alive cells were calculated for each culture. Within each experiment (that included cultured neurons from the brains of the same individuals) the values were normalized to survival in the untreated control culture ( $\hat{=} 100 \%)$. Differences in data population distributions were probed with the non-parametric Kruskal-Wallis test, and the Mann-Whitney $U$ test for unmatched samples was used to determine the differences between two groups (statistics performed with OriginPro 8.5, OriginLab Corporation). To adjust the false discovery rate, $P$ values were corrected with the BenjaminiHochberg procedure (Benjamini and Hochberg, 1995; Groppe et al., 2011).

\section{RESULTS}

\section{Sequence Analysis}

The T. castaneum gene prediction au3.g2971.t1 (Tc000209, Tc-crlf3) is an ortholog of the human crlf3. It is located on chromosome linkage group 2 (ChLG2) at position 14179075-14181074. The size of the mRNA is $1803 \mathrm{bp}$ and the coding sequence, distributed over five exons, comprises 1272 bp. Conserved domains of the 424 amino acid containing Tc-CRLF3 protein include a fibronectin type III domain, a cytokine receptor motif and interdomain contacts (Supplementary Figure S2).

A phylogenetic tree revealed clear orthology of human, mouse and Tribolium CRLF3 proteins (Figure 1A). Importantly, no further paralogs were detected in Tribolium which could act redundantly and possibly rescue RNAi-induced phenotypes (Figure 1A).

CRLF3 orthologs are present in mammals, amphibia, fish, cephalochordates, tunicates and insects. Among insects, crlf3 

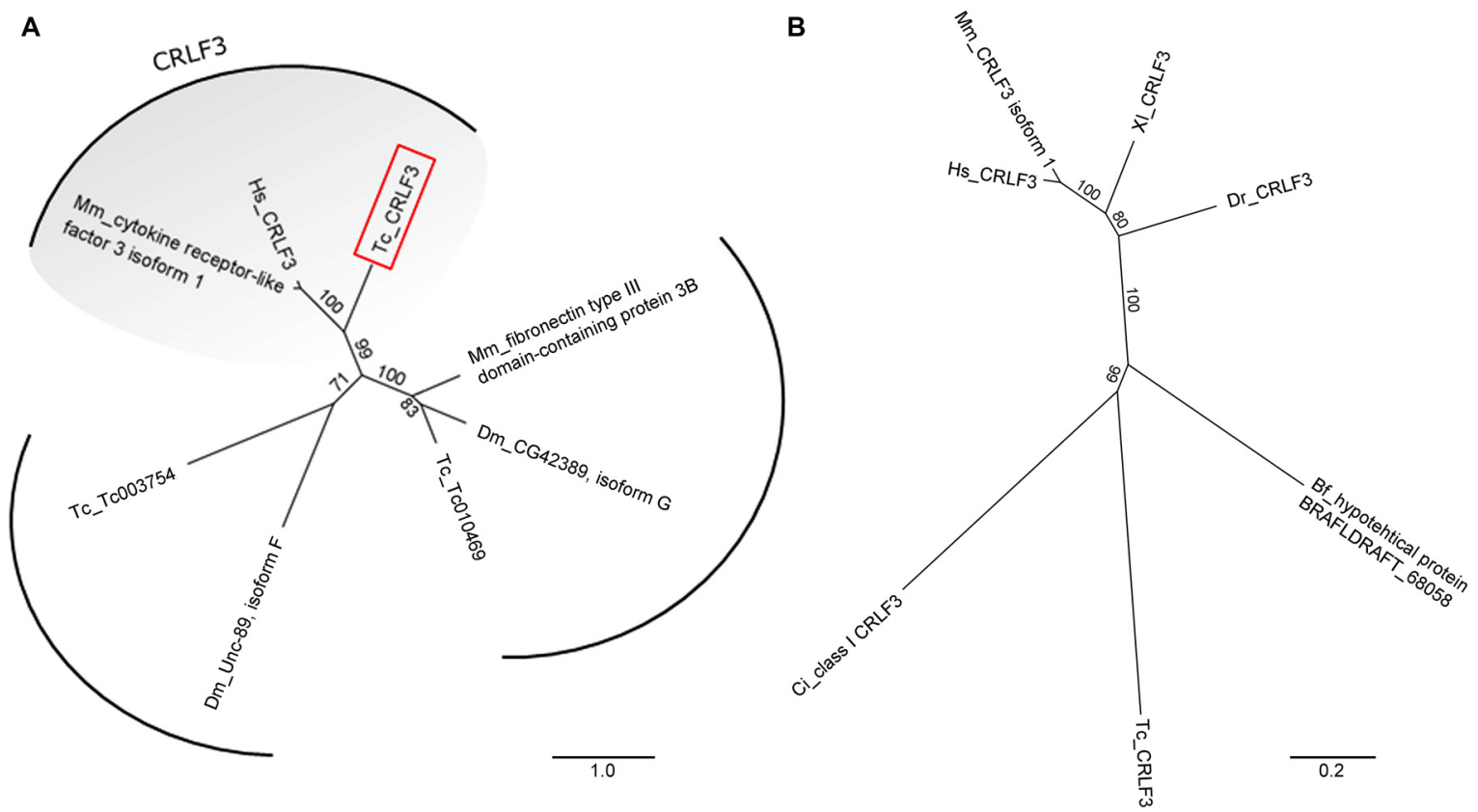

FIGURE 1 | Unrooted phylogenetic trees showing the relationship of cytokine receptor-like factor 3 (CRLF3) proteins. (A) Phylogenetic tree originating from Basic Local Alignment Search Tool (BLAST) with the human CRLF3 protein sequence (Hs_CRLF3). Protein sequences from Tribolium castaneum (Tc), Drosophila melanogaster (Dm) and Mus musculus (Mm) were considered for the alignment. Tribolium Tc-CRLF3 (red box) is an ortholog of the human CRLF3 since it belongs to the CRLF3 group. Paralogs are not present in Tribolium. (B) CRLF3 orthologs of Homo sapiens (Hs), Mus musculus (Mm), Danio rerio (Dr), Xenopus laevis (XI), Branchiostoma floridae (Bf), Ciona intestinalis (Ci) and Tribolium castaneum (Tc) were compared with respect to phylogenetic relationship. Orthologs of mammalian as well as of vertebrate CRLF3 separate in well supported branches. Table 2 summarizes the identity of the respective proteins.

TABLE 1 | Sequences of primers.

\section{Primer}

crlf3 fragment 1 forward

crlf3 fragment 1 reverse

crlf3 fragment 2 forward

crlf3 fragment 2 reverse

$\mathrm{M} 13 \mathrm{~F}(-20)$

T7-M13R (-17)

T7-pJet1.2 forward

T7-pJet1.2 reverse

\section{Sequence}

5' GGGTGATAGGAACGAAGTGGTGGA 3'

5' GACGTCATATCTGAGAAACACTTAG 3'

5' CGATTGTTATGTGGGCGCAGAGAC 3'

5' GAGTCAGTATTGATACGTGTAACA 3'

5' GTAAAACGACGGCCAGT 3'

5' TAATACGACTCATAGGCAGGAAACAGCTATGAC 3'

5' TAATACGACTCACTATAGGCGACTCACTATAGGGAGAGC 3'

5' TAATACGACTCACTATAGGAAGAACATCGATIITCCATGGCAG 3' genes were identified in the beetle T. castaneum and the cricket Gryllus bimaculatus ( ${ }^{1}$ Gene: GB-isotig00932) but not in fruit flies, mosquitoes and honey bees (Wyder et al., 2007). On the protein level, Tc-CRLF3 shares $28 \%$ sequence similarity with its human and mouse orthologs, $29 \%$ with $X$. laevis and $26 \%$ with zebrafish (Table 2). Respective proteins of the lancelet B. floridae and the tunicate C. intestinales share $27 \%$ and $24 \%$ similarity with Tc-CRLF3. Analysis of phylogenetic relationships among CRLF3 proteins of these species reflects the evolutionary lineages (Figure 1B). Orthologs of mammalian (mouse and human) as well as of vertebrate (mammals together with frog and fish) CRLF3 form branches that are well-separated from the other species. The non-vertebrate species differ significantly from each other, with CRLF3 of Tribolium showing

\footnotetext{
${ }^{1}$ http://asgard.rc.fas.harvard.edu/blast.html
}

stronger similarity with Ciona than with the other chordate Branchiostoma.

\section{Primary Cell Culture from Brains of $T$. Castaneum}

Freshly dissociated primary cell cultures contained round shaped cells that were typically devoid of processes (Figure 2A). Neurons and eventually also glia lose their axons, dendrites and other processes during the dissociation procedure. It is assumed that a large portion of cells is irreversibly damaged during this process preventing their attachment to the concanavalin A-coated cover slips and subsequent survival in vitro. After 1 day, cultures in full medium with 5\% FBS contained approximately $65 \%$ alive cells while survival in serum-free cultures was reduced to approximately $40 \%$ of adherent cells. Primary cultured neurons regenerated neurites that became visible after 3 days 
TABLE 2 | Across species similarities of amino acid sequences of cytokine receptor-like factor 3 (CRLF3) orthologs.

\begin{tabular}{|c|c|c|c|c|c|c|c|}
\hline & $\begin{array}{l}\text { H. s. } \\
\text { CRLF3 }\end{array}$ & $\begin{array}{l}\text { M. } m \text {. CRLF3 } \\
\text { isoform } 1\end{array}$ & $\begin{array}{c}\text { D. } r . \\
\text { CRLF3 }\end{array}$ & $\begin{array}{c}\text { X. I. } \\
\text { CRLF3 }\end{array}$ & $\begin{array}{c}\text { B. } f \text {. hyp. protein } \\
\text { BRAFLDRAFT_68058 }\end{array}$ & $\begin{array}{l}\text { C. i. class I } \\
\text { CRLF3 }\end{array}$ & $\begin{array}{c}\text { T. c. } \\
\text { CRLF3 }\end{array}$ \\
\hline H. sapiens & 442 aa & $93 \%$ & $66 \%$ & $70 \%$ & $34 \%$ & $28 \%$ & $28 \%$ \\
\hline M. musculus & $93 \%$ & 442 aа & $65 \%$ & $70 \%$ & $33 \%$ & $28 \%$ & $28 \%$ \\
\hline D. rerio & $66 \%$ & $65 \%$ & 444 aа & $59 \%$ & $36 \%$ & $28 \%$ & $26 \%$ \\
\hline$X$. laevis & $70 \%$ & $70 \%$ & $59 \%$ & 436 aа & $35 \%$ & $28 \%$ & $29 \%$ \\
\hline B. floridae & $34 \%$ & $33 \%$ & $36 \%$ & $35 \%$ & 441 aа & $30 \%$ & $28 \%$ \\
\hline C. intestinalis & $28 \%$ & $28 \%$ & $28 \%$ & $28 \%$ & $30 \%$ & 463 aа & $24 \%$ \\
\hline T. castaneum & $28 \%$ & $28 \%$ & $26 \%$ & $29 \%$ & $27 \%$ & $24 \%$ & 424 aa \\
\hline
\end{tabular}

Shaded cells contain total numbers of amino acids of respective proteins. GenBank accession numbers of sequences: H. sapiens (human) NP_057070.3; M. musculus (mouse) NP_061246.1; D. rerio (zebrafish) NP_001017817.2; X. laevis (frog) NP_001080600.1; B. floridae (lancelet) X P_002607148.1; C. intestinalis (tunicate) NP_001107600.1; T. castaneum (beetle) XP_008190449.1.
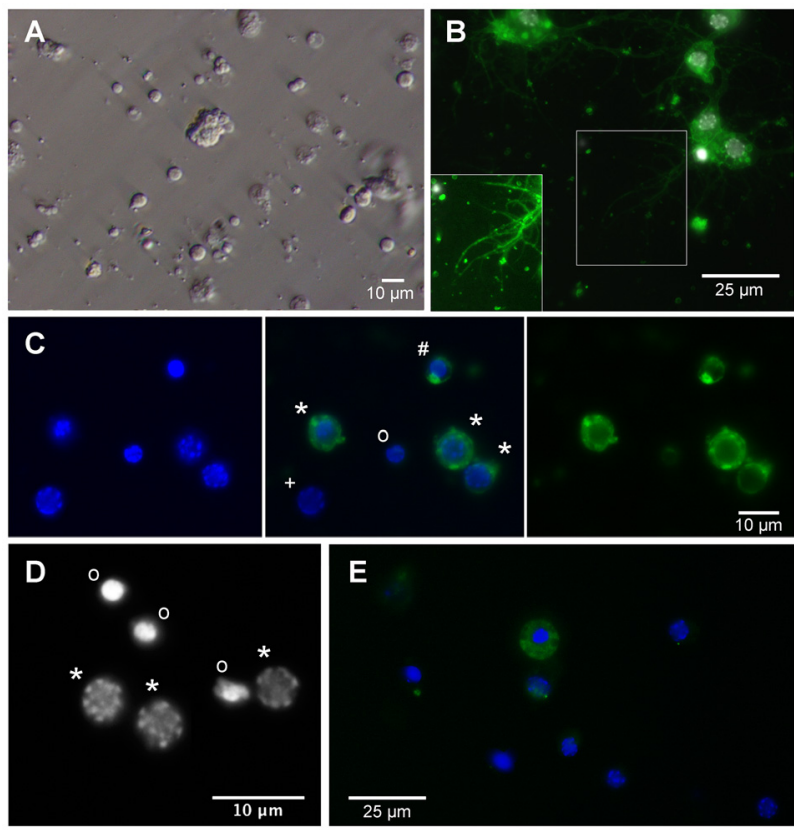

FIGURE 2 | Primary cell culture from T. castaneum brains. (A) Cultured brain ells $3 \mathrm{~h}$ after dissociation. (B) Cultured brain neurons labeled with anti-horseradish peroxidase (anti-HRP) (green) and DAPI (white) after 6 days in vitro. Inset shows regenerated neurites with optimized brightness and contrast. (C) Cultured brain cells after 1 day in vitro. Left and middle: DAPI labeling. Right and middle: anti-HRP immunofluorescence. *Intact neurons, \# dying neuron, ${ }^{\circ}$ nucleus of dead or dying cell, + intact glial cell. (D) DAPI labels heterochromatin DNA structures in nuclei of intact cells $\left(^{*}\right)$ and condensed nuclei with largely fragmented DNA of dead or dying cells (O). (E) Anti-cleaved caspase-3 immunofluorescence (green) in the cytoplasm of apoptotic cells. Dead cells (condensed DAPI-labeled nuclei) lose their cleaved caspase-3 associated fluorescence after disintegration of cellular membranes. Activated caspase- 3 is absent from the cytoplasm of intact cells with discontinuously DAPI-labeled nuclei.

in vitro and subsequently increased in length and complexity of arborizations (Figure 2B). Whether anatomical contacts of processes developed into functional synapses was not studied. Neurons and glia were distinguished by the presence or absence of anti-HRP immunoreactivity (Figure 2C). Anti-HRP has been demonstrated to label neuron-specific membrane proteins in various arthropods and anti-HRP immunocytochemistry is a simple, reliable and widely used method to identify insect neurons (Jan and Jan, 1982; Sun and Salvaterra, 1995; Gocht et al., 2009). However, since cytoplasmic membranes disintegrate in dying or dead cells, anti-HRP immunocytochemistry cannot distinguish between dead neurons and glia (e.g., the nucleus marked with "o" in the center of Figure 2C). As it was previously described for primary cultures from locust brains (Vanhems and Delbos, 1987; Gocht et al., 2009), fresh cultures from $T$. castaneum brains contained low numbers of glia and no glial cells were detected after 4 days in vitro. Viability of primary cultured cells was reflected in the pattern of the DNA-binding fluorescent dye DAPI (Figure 2D). Nuclei of intact cells (both neurons and glia, Figure 2C) display a patchy staining pattern that corresponds to intact chromatin structures. DNA fragmentation, that goes along with apoptotic cell death, leads to condensed and uniformly DAPI-labeled nuclei. Apoptotic cell death in cultured insect neurons can be induced by serumdeprivation, hypoxia and other physiological stressors (Jenkins et al., 2013; Miljus et al., 2014). Caspase activation involved in apoptosis can be detected in T. castaneum brain cell cultures by anti-cleaved caspase-3 immunocytochemistry (Figure 2E).

\section{Epo and EV-3 Increase the Survival of T. Castaneum Brain Neurons}

Cellular survival in untreated control cultures and cultures exposed to different concentrations of rhEpo or EV-3 was assessed after 7 days in vitro. Pharmacological treatment started after 4 days in vitro, when cultures were devoid of glia and exclusively contained neurons that could be labeled with anti-HRP immunocytochemistry (Figures 2B,C). rhEpo concentrations of 0.8 and $3.2 \mathrm{ng} / \mathrm{ml}$ significantly increased neuronal survival in cultures deriving from identical pools of brain cells (both with $p<0.001$ compared to controls) while $32 \mathrm{ng} / \mathrm{ml}$ rhEpo reduced viability ( $p<0.05$ compared to control; Figure 3A). The human non-erythropoietic Epo splice variant EV-3 also significantly $(p<0.05$ for $0.42 \mathrm{ng} / \mathrm{ml} \mathrm{EV-3}$ and $p<0.01$ for $0.84 \mathrm{ng} / \mathrm{ml} \mathrm{EV}-3$ compared to control) increased the survival of T. castaneum brain neurons in vitro (Figure 3B).

\section{RNAi in Cultured Brain Neurons}

RNAi by application of dsRNA to the extracellular space is frequently described as soaking RNAi. In order to test, whether 


\section{A}
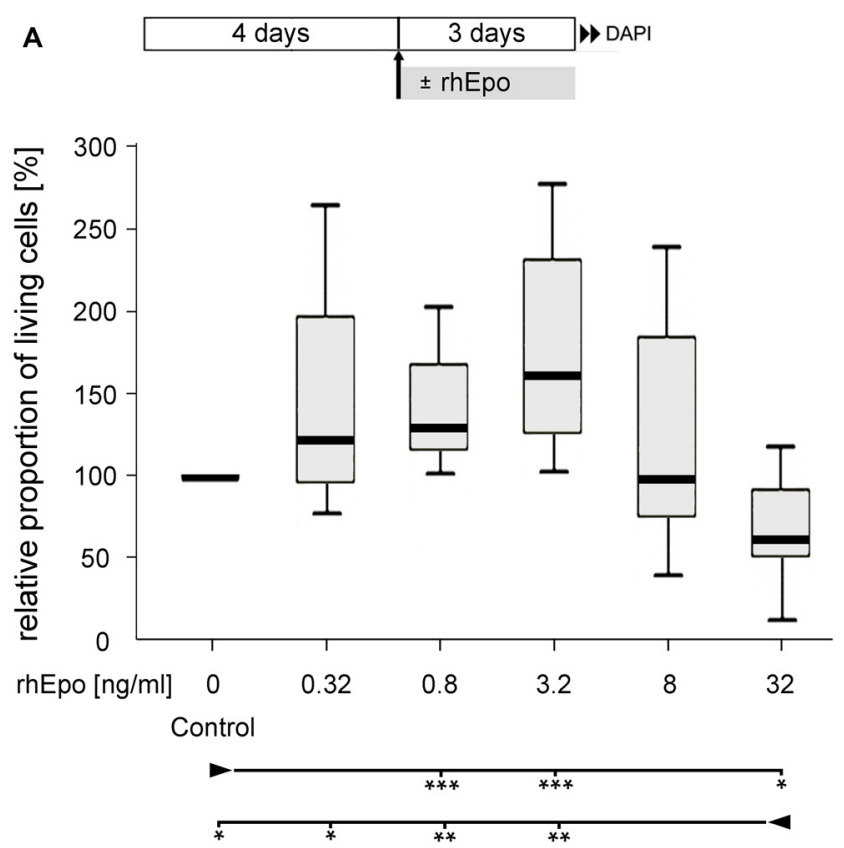

B
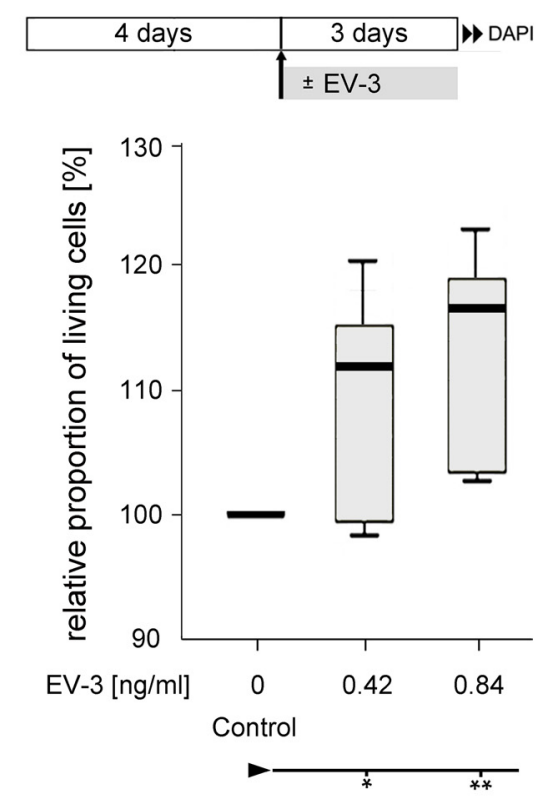

FIGURE 3 | Effect of recombinant human Epo (rhEpo) and EV-3 on cellular survival of T. castaneum primary brain cell cultures after 7 days in vitro. (A) In comparison to untreated control cultures 0.8 and $3.2 \mathrm{ng} / \mathrm{ml} \mathrm{rhEpo}$ significantly increase cellular survival ( $p=0.00088$ for both). In contrast, $32 \mathrm{ng} / \mathrm{ml}$ rhEpo decreases cellular survival compared with untreated control cultures $(p=0.01202)$ and cultures exposed to lower concentrations of rhEpo $(0.32 \mathrm{ng} / \mathrm{ml}: p=0.02603,0.8 \mathrm{ng} / \mathrm{ml}$ : $p=0.00233,3.2 \mathrm{ng} / \mathrm{ml}: p=0.00233) . N=8,64,518$ cells evaluated. (B) EV-3 increases cellular survival $(0.42 \mathrm{ng} / \mathrm{ml}: p=0.04762 ; 0.84 \mathrm{ng} / \mathrm{ml}: p=0.00794)$. $N=7$, 42,355 cells evaluated Statistics: non-parametric Kruskal-Wallis test and Mann-Whitney $U$ test with Benjamini-Hochberg correction. ${ }^{* * *} p<0.001,{ }^{* *} p<0.01$, ${ }^{*} p<0.05$. Significance is shown with respect to the treatment group marked by the arrowhead. Schematics in the upper part describe experimental procedure with initial 4 days of culturing in full medium and 3 days of rhEpo or EV-3 exposure.

the mechanisms underlying dsRNA uptake and RNAi are retained in cultured brain cells, dsRNA fragments targeting the expression of three different proteins (one that does not exist in T. castaneum, one that is essential for cellular survival and two fragments targeting Tc-CRLF3) were applied to the culture medium for 4 days. DsRNA targeting the expression of Rpt3 dramatically reduced cellular survival in comparison to untreated control cultures that derived from the same pools of brains (Figures 4A,B), indicating effective dsRNA uptake and knockdown of expression of this essential proteasomal protein. In contrast, cell survival was not affected by dsRNA targeting dsRed, a protein that is not naturally expressed in insect cells (Figure 4A). Thus, uptake of dsRNA per se did not compromise the viability of cultured brain cells. Similarly, dsRNA targeting the expression of Tc-CRLF3 (fragment 2: Figure 4A and fragment 1: Figure 4B) had no impact on cellular survival, suggesting that the reduction of Tc-CRLF3 expression is not critical for cell survival under normal culture conditions.

\section{Knockdown of TC-CRLF3 Expression Abolishes the Neuroprotective Effect of rhEpo}

In order to evaluate the role of Tc-CRLF3 in Epo-mediated neuroprotection, we performed two independent series of experiments (experimental procedure shown in Figure 5A) in which Tc-CRLF3 expression was suppressed by interference with two different dsRNAs, targeting different, non-overlapping fragments of the transcript (fragment 1 and fragment 2). Exposure to hypoxia for $36 \mathrm{~h}$ significantly reduced cellular survival in comparison to cultures that were maintained under normoxic conditions (Figure 5B, $p<0.001$; Figure 5C, $p<0.001)$. Incubation with $0.8 \mathrm{ng} / \mathrm{ml} \mathrm{rhEpo}$ completely prevented hypoxia-induced cell death (Figure 5B, $p<0.001$; Figure 5C, $p<0.001$ ). Since all cultures were maintained in serum-free medium, which acts as a mild stressor for cellular survival, survival in rhEpo-treated/hypoxia-exposed cultures was even higher than in normoxic controls. RNAi-mediated knockdown of Tc-CRLF3 expression completely abolished the neuroprotective effect of rhEpo in hypoxia-exposed neuronal cultures. RNAi targeting both fragment 1 (Figure 5B) and fragment 2 (Figure 5C) significantly decreased cellular survival compared to rhEpo-treated/hypoxia-exposed cultures (both with $p<0.001$ ) and normoxic controls (both with $p<0.001$ ). Tc-CRLF3-directed RNAi had no additional deleterious effect on hypoxia-exposed neuronal cultures (Figures 5B,C) which is in line with our control experiment where Tc-crlf3 RNAi did not change survival in normal cell cultures (Figure 4). Thus, knockdown of Tc-CRLF3 expression eliminates Epo-mediated neuroprotection of $T$. castaneum brain neurons, without compromising cellular survival under neither normoxic (Figures 4A,B) nor hypoxic (Figures 5B,C) conditions. This 

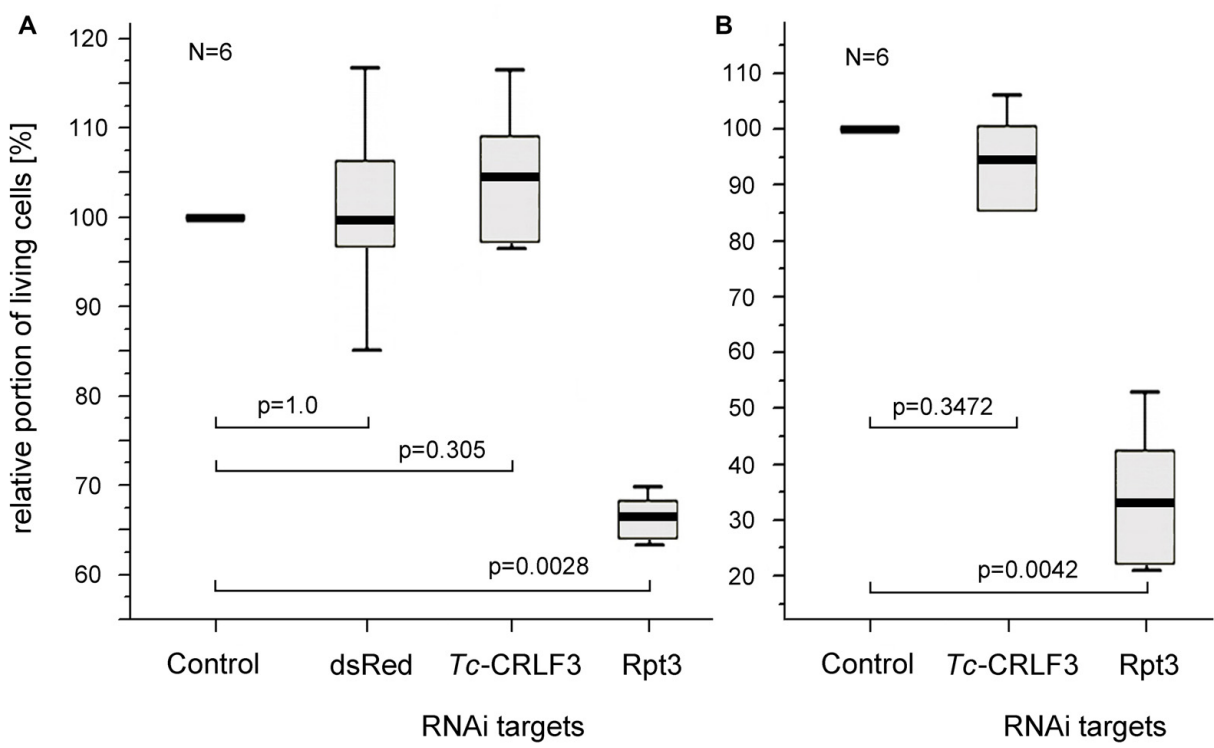

FIGURE 4 | Soaking RNAi in primary cultures of T. castaneum brain cells. (A) Cellular survival after 4 days in culture medium without serum (control) and supplemented with $10 \mathrm{ng} / \mu$ l double stranded RNA (dsRNA) to suppress expression of the anemonal protein dsRed that is not naturally expressed in insects, TC-CRLF3 (fragment 2) and the proteasomal protein Rpt3. $N=6,60,498$ cells evaluated. (B) Cellular survival after 4 days in culture medium without serum (control) and supplemented with $10 \mathrm{ng} / \mu \mathrm{l}$ dsRNA to suppress expression of TC-CRLF3 (fragment 1) and the proteasomal protein Rpt3. $N=6,66,505$ cells evaluated. Proportions of surviving cells were normalized to control cultures from the same pool of brain cells without dsRNA supplement (=100\%). Statistics: non-parametric Kruskal-Wallis test and Mann-Whitney $U$ test with Benjamini-Hochberg correction.

confirms the assumption that Tc-CRLF3 is the receptor mediating Epo neuroprotective effects in T. castaneum.

\section{DISCUSSION}

Cytokines and their receptors coordinate many physiological processes that adapt organisms to changing environments and maintain cellular functionality under unfavorable conditions. Cytokines include a heterogeneous collection of glycoproteins that typically activate multiple types of receptors. Cytokine receptors, typically hetero- or homo-multimeric complexes, can be activated by multiple different cytokine ligands (review: Dinarello, 2007). Overlapping and complementary functions of cytokines together with low primary sequence similarities of cytokines and many types of cytokine receptors complicate across-species comparison and studies on their evolutionary origins (Beschin et al., 2001; Liongue and Ward, 2007). As an exception, CRLF3 is phylogenetically well-conserved and orthologs were detected in mammals, frog, fish, non-vertebrate chordates and some, but not all insects (this study; Wyder et al., 2007). CRLF3 has been classified as a type I cytokine receptor of group 1 ("prototypic type I cytokine receptors") that also includes thrombopoietin receptor, prolactin receptor, growth hormone receptor and classical EpoR (Boulay et al., 2003). Cytokine receptors of this group are single pass transmembrane receptors that associate to mono- or heteromeric complexes and transduce their activation into the cell through interaction with JAKs.
We established adherent primary cell cultures from brains of $T$. castaneum. Culture conditions supported the survival of neurons and eliminated glial cells within 4 days in vitro. Similar observations were previously made with primary brain cell cultures form L. migratoria (Gocht et al., 2009). Successful RNAi-mediated downregulation of protein expression has previously been demonstrated in various developmental stages of T. castaneum (Bucher et al., 2002; Ulrich et al., 2015). In this study we extended the application of RNAi to T. castaneum-derived neuronal cell cultures, by showing that expression of particular proteins (such as rpt3 and Tc-CRLF3) can be suppressed by soaking dsRNAi.

Epo promoted the survival of primary neuron cultures from T. castaneum brains and effectively suppressed apoptotic cell death induced by hypoxia and serum deprivation. This confirmed the results from previous studies with primary neuronal cultures from locust brains (Ostrowski et al., 2011; Miljus et al., 2014) and suggested that Epo-mediated neuroprotection might be a common mechanism in those insects that contain orthologs of CRLF3 (e.g., T. castaneum, L. migratoria, G. bimaculatus but not D. melanogaster). RNAi-mediated knockdown of Tc-CRLF3 expression completely abolished the neuroprotective effect of Epo, indicating that this cytokine receptor is essential for initiating anti-apoptotic mechanisms in $T$. castaneum brain neurons. In order to minimize the risk that suppressed expression of another protein besides Tc-CRLF3 interferes with Epo-mediated neuroprotection, Tc-CRLF3 expression was knocked down with two different dsRNA constructs that targeted different, 


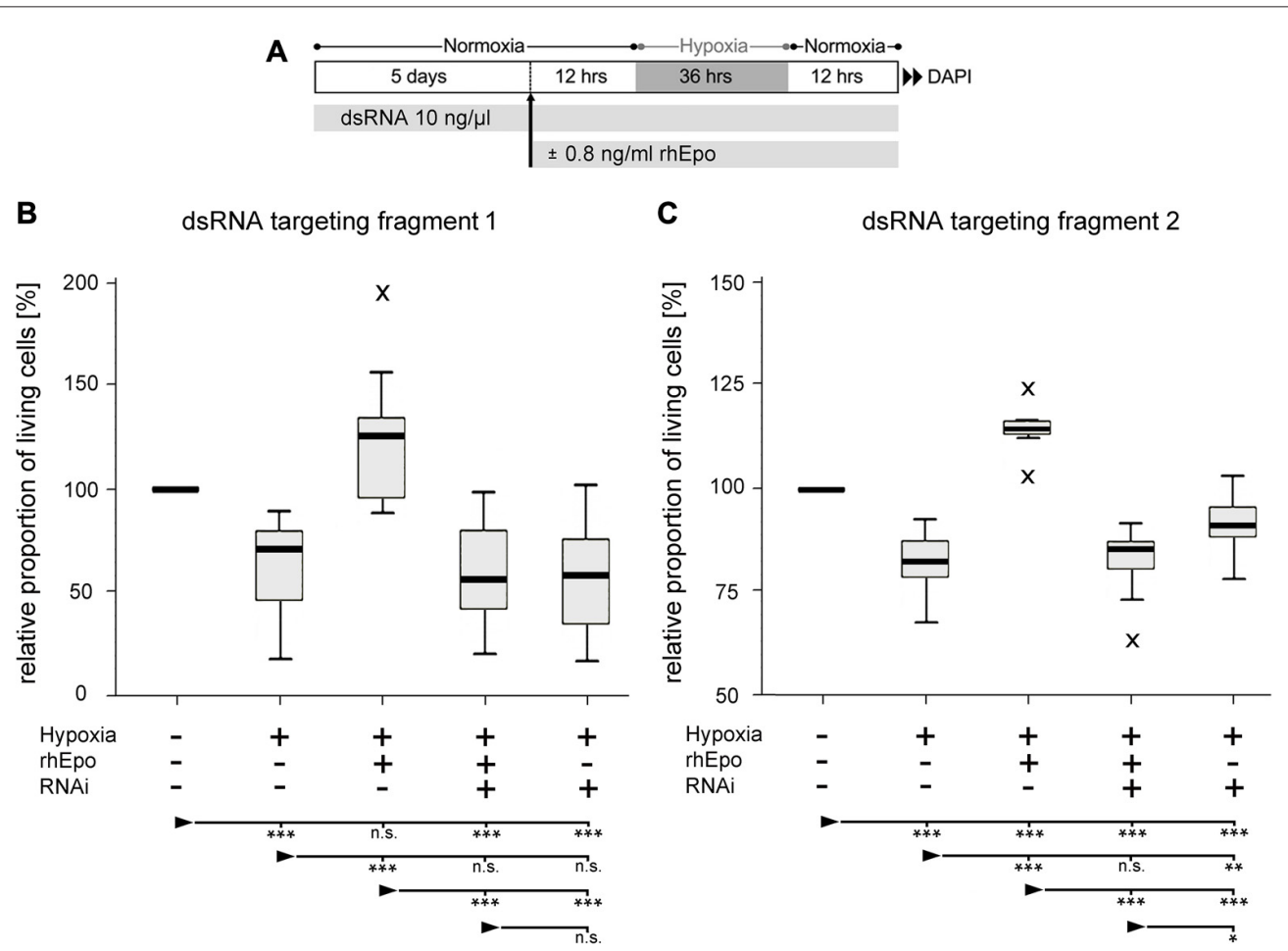

FIGURE 5 | Downregulation of CRLF3 expression abolishes rhEpo-mediated neuroprotection of hypoxia-exposed neurons. (A) Schematic representation of experimental procedure. (B) RNAi with dsRNA fragment 1 of the TC-CRLF3 transcript. $N=16,127,808$ cells evaluated. (C) RNAi with dsRNA targeting fragment 2 of the TC-CRLF3 transcript. $N=14,134,200$ cells evaluated. Statistics: non-parametric Kruskal-Wallis test and Mann-Whitney $U$ test for unmatched samples. $P$ values were corrected with the Benjamini-Hochberg procedure. ${ }^{* * *} p<0.001,{ }^{* *} p<0.01,{ }^{*} p<0.05$, n.s. not significant. Significance is shown with respect to the treatment group marked by the arrowhead.

entirely non-overlapping sequences of the Tc-crlf3 gene transcript. Both dsRNA constructs abolished Epo-mediated protection of hypoxia-exposed beetle neurons. Complete loss of Epo-mediated neuroprotection was previously achieved by co-incubating hypoxia-exposed locust brain neurons with JAK and STAT inhibitors (Miljus et al., 2014), suggesting that Tc-CRLF3 is an upstream component of this neuroprotective transduction pathway. This is in line with the presence of predicted JAK docking sites in $T$. castaneum and human CRLF3 (this study; Boulay et al., 2003) and the observation that human CRLF3 activates STAT3 (Yang et al., 2009). However, Tc-CRLF3-directed RNAi did not compromise neuronal survival in normoxic and hypoxia-exposed neuronal cultures, suggesting that it is not essential for cellular survival, though being indispensable for Epo-mediated neuroprotection. Similarly, studies on PC12 and HEK cells have not reported any obvious decrease in viability following CRLF3 knockdown (Yang et al., 2009; Hashimoto et al., 2012).

Epo-mediated neuroprotection shares a number of common mechanisms between mammals (mostly studied in rodents) and insects (studied in locusts and beetles). In both groups of animals neuroprotection is mediated via JAK and STAT activation, while mammalian neurons may additionally employ transduction pathways involving $\mathrm{MAPK}, \mathrm{PI} 3 \mathrm{~K}$ and $\mathrm{NF}_{\mathrm{K}} \mathrm{B}$ (Digicaylioglu and Lipton, 2001; Sirén et al., 2001; Miljus et al., 2014).
Various studies demonstrated optimum-type dose-response curves for different concentrations of experimentally applied Epo (this study; Sirén et al., 2001; Weishaupt et al., 2004; Ostrowski et al., 2011; Miller et al., 2015) and Epo-induced receptor endocytosis has been described for locust neurons (Miljus et al., 2017) and mammalian erythroid progenitors (Bulut et al., 2011). Importantly, the non-erythropoietic splice variant EV-3 increased the survival of serum-deprived primary cultured $T$. castaneum brain neurons (this study) and protected both hypoxia-exposed locust brain neurons (Miljus et al., 2017) and rat cortex neurons challenged with glucose and oxygen deprivation (Bonnas, 2012; Bonnas et al., 2017) from apoptotic cell death. This suggested a greater similarity of the ligand binding regions of locust and mammalian neuroprotective Epo receptors than between mammalian neuroprotective and classical erythropoietic EpoR.

CRLF3 has been implicated with typical cytokine-regulated processes such as cell cycling and cellular morphogenesis (Yang et al., 2009; Hashimoto et al., 2012) and alterations of its expression have been detected in various types of tumors (Dang et al., 2006; Yang et al., 2009) and patients affected by ALS (Cirulli et al., 2015). However, the detailed cellular function of CRLF3 has not been resolved and descriptions of its functional domains and its subcellular 
localization are inconsistent. The present study deorphanizes CRLF3 by identifying Epo-like signaling molecules as its ligand. Preliminary own studies detected CRLF3 expression in mouse brain, liver and kidney, suggesting that it mediates functions in large parts of mammalian organisms. Whether it functions as a neuroprotective or, more general, as a cytoprotective Epo receptor in mammals including humans needs to be studied by appropriate assays. Insects, such as T. castaneum, but not Drosophila melanogaster that lack both a CRLF3 ortholog and Epo-mediated neuroprotection, may be used to search for additional tissue-protective Epo receptors with conserved orthologs. Since insects lack erythrocytes and "classical" EpoR, studies do not have to consider interference of these mechanisms. Moreover, as insects do not contain epo genes that can be identified by sequence similarity, an "alternative" ligand awaits to be identified that activates insect neuroprotective receptors such as CRLF3 and may have mammalian orthologs with similar functions.

\section{AUTHOR CONTRIBUTIONS}

$\mathrm{NH}, \mathrm{DYK}, \mathrm{JL}, \mathrm{KP}, \mathrm{MB}$ and $\mathrm{RH}$ performed experiments and analyzed data. NH, LW, MB, GB, HE and RH designed the study and wrote the manuscript.

\section{REFERENCES}

Alnaeeli, M., Wang, L., Piknova, B., Rogers, H., Li, X., and Noguchi, C. T. (2012). Erythropoietin in brain development and beyond. Anat. Res. Int. 2012:953264. doi: 10.1155/2012/953264

Altschul, S. F., Gish, W., Miller, W., Myers, E. W., and Lipman, D. W. (1990). Basic local alignment search tool. J. Mol. Biol. 215, 403-410. doi: 10.1006/jmbi.19 90.9999

Arcasoy, M. O. (2008). The non-haematopoietic biological effects of erythropoietin. Br. J. Haematol. 141, 14-31. doi: 10.1111/j.1365-2141.2008. 07014.x

Benjamini, Y., and Hochberg, Y. (1995). Controlling the false discovery rate: a practical and powerful approach to multiple testing. J. Royal Statist. Soc. B 57, 289-300.

Beschin, A., Bilej, M., Torreele, E., and De Baetselier, P. (2001). On the existence of cytokines in invertebrates. Cell. Mol. Life Sci. 58, 801-814. doi: $10.1007 / \mathrm{pl} 00000901$

Bonnas, C. B. (2012). Identification of Erythropoietin Isoforms and Evaluation of Their Biological Importance. Berlin: PhD thesis, Medical Faculty Charité. Available online at: http://www.diss.fu-berlin.de/diss/receive/FUDISS_thesis_ 000000009488

Bonnas, C., Wüstefeld, N., Winkler, D., Kronstein-Wiedemann, R., Dere, E., Specht, K., et al. (2017). EV-3, an endogenous human erythropoietin isoform with distinct functional relevance. Sci. Rep. 7:3684. doi: 10.1038/s41598-01703167-0

Boulay, J. L., O'Shea, J. J., and Paul, W. E. (2003). Molecular phylogeny within type I cytokines and their cognate receptors. Immunity 19, 159-163. doi: 10.1016/s1074-7613(03)00211-5

Brines, M., and Cerami, A. (2005). Emerging biological roles for erythropoietin in the nervous system. Nat. Rev. Neurosci. 6, 484-494. doi: 10.1038/ nrn1739

Bucher, G., Scholten, J., and Klingler, M. (2002). Parental RNAi in Tribolium (Coleoptera). Curr. Biol. 12, R85-R86. doi: 10.1016/s0960-9822(02)00666-8

Bulut, G. B., Sulahian, R., Ma, Y., Chi, N., and Huang, L. N. (2011). Ubiquitination regulates the internalization, endosomal sorting, and signaling

\section{ACKNOWLEDGMENTS}

We thank Elke Küster for maintenance of beetle cultures and Claudia Hinners and Julia Ulrich for the production of dsRNA. We also thank Margret Winkler, Silvia Gubert, Nicola Schwedhelm-Domeyer and Stephanie Pauls for technical assistance. We acknowledge the generous support by the Open Access Publication Funds of the Göttingen University.

\section{SUPPLEMENTARY MATERIAL}

The Supplementary Material for this article can be found online at: http://journal.frontiersin.org/article/10.3389/fnmol. 2017.00223/full\#supplementary-material

FIGURE S1 | 5' to 3' strand of double stranded RNA (dsRNA) fragments used for RNAi in primary brain cell cultures from $T$. castaneum.

FIGURE S2 | Aligned amino acid sequences of human cytokine receptor-like factor 3 (CRLF3; NCBI GenBank NP_057070.3) and orthologous au3.g2971.t1-derived protein Tc-CRLF3 from T.castaneum. Predicted domains are indicated by bars on top of the sequence. Domain information related to human CRLF3 derived from $\mathrm{NCBI}$ (http://www.ncbi.nlm.nih.gov/protein/Q8IUI8.2) and (24).

of the erythropoietin receptor. J. Biol. Chem. 8, 6449-6457. doi: 10.1074/jbc. M110.186890

Chateauvieux, S., Grigorakaki, C., Morceau, F., Dicato, M., and Diederich, M. (2011). Erythropoietin, erythropoiesis and beyond. Biochem. Pharmacol. 82, 1291-1303. doi: 10.1016/j.bcp.2011.06.045

Cirulli, E. T., Lasseigne, B. N., Petrovski, S., Sapp, P. C., Dion, P. A., Leblond, C. S., et al. (2015). Exome sequencing in amyotrophic lateral sclerosis identifies risk genes and pathways. Science 347, 1436-1441. doi: 10.1126/science. aaa3650

Collino, M., Thiemermann, C., Cerami, A., and Brines, M. (2015). Flipping the molecular switch for innate protection and repair of tissues: long-lasting effects of a non-erythropoietic small peptide engineered from erythropoietin. Pharmacol. Ther. 151, 32-40. doi: 10.1016/j.pharmthera. 2015.02.005

Dang, C., Gottschling, M., Manning, K., O’Currain, E., Schneider, S., Sterry, W., et al. (2006). Identification of dysregulated genes in cutaneous squamos cell carcinoma. Oncol. Rep. 16, 513-519. doi: 10.3892/or.16.3.513

Digicaylioglu, M., and Lipton, S. A. (2001). Erythropoietin-mediated neuroprotection involves cross-talk between Jak2 and NF-кB signalling cascades. Nature 412, 641-647. doi: 10.1038/35088074

Dinarello, C. A. (2007). Historical insights into cytokines. Eur. J. Immunol 37, S34-S45. doi: 10.1002/eji.200737772

Duedal Rölfing, J. H., Baatrup, A., Stiehler, M., Jensen, J., Lysdahl, H., and Bünger, C. (2014). The osteogenic effect of erythropoietin on human mesenchymal stromal cells is dose-dependent and involves non-hematopoietic receptors and multiple intracellular signalling pathways. Stem Cell Rev. 10, 69-78. doi: 10.1007/s12015-013-9476-x

Erbayraktar, S., Grasso, G., Sfacteria, A., Xie, Q. W., Coleman, T., Kreilgaard, M., et al. (2003). Asialoerythropoietin is a nonerythropoietic cytokine with broad neuroprotective activity in vivo. Proc. Natl. Acad. Sci. US A 100, 6741-6746. doi: 10.1073/pnas.1031753100

Gan, Y., Xing, J., Jing, Z., Stetler, R. A., Zhang, F., Luo, Y., et al. (2012). Mutant erythropoietin without erythropoietic activity is neuroprotective against ischemic brain injury. Stroke 43, 3071-3077. doi: 10.1161/STROKEAHA.112. 663120 
Gocht, D., Wagner, S., and Heinrich, R. (2009). Recognition, presence and survival of locust central nervous glia in situ and in vitro. Microsc. Res. Tech. 72, 385-397. doi: 10.1002/jemt.20683

Groppe, D. M., Urbach, T. P., and Kutas, M. (2011). Mass univariate analysis of event-related brain potentials/fileds I: a critical tutorial review. Psychophysiology 48, 1711-1725. doi: 10.1111/j.1469-8986.2011. 01273.x

Hashimoto, Y., Muramatsu, K., Kunii, M., Yoshimura, S., Yamada, M., and Sato, T. (2012). Uncovering genes required for neuronal morphology by morphologybased gene trap screening with a revertible retrovirus vector. FASEB J. 26, 4662-4674. doi: 10.1096/fj.12-207530

Jan, L. Y., and Jan, Y. N. (1982). Antibodies to horseradish peroxidase as specific neuronal markers in Drosophila and in grasshopper embryos. Proc. Natl. Acad. Sci. U S A 79, 2700-2704. doi: 10.1073/pnas.79.8.2700

Jelkmann, W. (1992). Erythropoietin: structure, control of production, and function. Physiol. Rev. 72, 449-489.

Jenkins, V. K., Timmons, A. K., and McCall, K. (2013). Diversity of cell death pathways: insight from the fly ovary. Trends Cell Biol. 23, 567-574. doi: 10.1016/j.tcb.2013.07.005

Kästner, A., Grube, S., El-Kordi, A., Stepniak, B., Friedrichs, H., Sargin, D., et al. (2012). Common variants of the genes encoding erythropoietin and its receptor modulate cognitive performance in schizophrenia. Mol. Med. 18, 1029-1040. doi: 10.2119/molmed.2012.00190

King, C. E., Rodger, J., Bartlett, C., Esmaili, T., Dunlop, S. A., and Beazley, L. D. (2007). Erythropoietin is both neuroprotective and neuroregenerative following optic nerve transection. Exp. Neurol. 205, 48-55. doi: 10.1016/ j.expneurol.2007.01.017

Kretz, A., Happold, C. J., Marticke, J. K., and Isenmann, S. (2005). Erythropoietin promotes regeneration of adult CNS neurons via Jak2/Stat3 and PI3K/AKT pathway activation. Mol. Cell. Neurosci. 29, 569-579. doi: 10.1016/j.mcn.2005. 04.009

Leist, M., Ghezzi, P., Grasso, G., Bianchi, R., Villa, P., Fratelli, M., et al. (2004). Derivatives of erythropoietin that are tissue protective but not erythropoietic. Science 305, 239-242. doi: 10.1126/science.1098313

Liongue, C., and Ward, A. (2007). Evolution of class I cytokine receptors. BMC Evol. Biol. 7:120. doi: 10.1186/1471-2148-7-120

Miljus, N., Heibeck, S., Jarrar, M., Micke, M., Ostrowski, D., Ehrenreich, H., et al. (2014). Erythropoietin-mediated protection of insect brain neurons involves JAK and STAT but not PI3K transduction pathways. Neuroscience 258, 218-227. doi: 10.1016/j.neuroscience.2013.11.020

Miljus, N., Massih, B., Weis, M. A., Rison, J. V., Bonnas, C. B., Sillaber, I., et al. (2017). Neuroprotection and endocytosis: erythropoietin receptors in insect nervous systems. J. Neurochem. 141, 63-74. doi: 10.1111/jnc. 13967

Miller, J. L., Church, T. J., Leonoudakis, D., Lariosa-Willingham, D., Frigon, N. L., Tettenborn, C. S., et al. (2015). Discovery and characterization of nonpeptidyl agonists of the tissue-protective erythropoietin receptor. Mol. Pharmacol. 88, 357-367. doi: 10.1124/mol.115.098400

Noguchi, C. T., Wang, L., Rogers, H. M., Teng, R., and Jia, Y. (2008). Survival and proliferative roles of erythropoietin beyond the erythroid lineage. Expert Rev. Mol. Med. 10:e36. doi: 10.1017/s14623994080 00860

Ostrowski, D., Ehrenreich, H., and Heinrich, R. (2011). Erythropoietin promotes survival and regeneration of insect neurons in vivo and in vitro. Neuroscience 188, 95-108. doi: 10.1016/j.neuroscience.2011.05.018

Sanchez, P. E., Fares, R. P., Risso, J. J., Bonnet, C., Bouvard, S., Le-Cavorsin, M., et al. (2009). Optimal neuroprotection by erythropoietin requires elevated expression of its receptor in neurons. Proc. Natl. Acad. Sci. U S A 106, 9848-9853. doi: 10.1073/pnas.0901840106
Sargin, D., El-Kordi, A., Agarwal, A., Müller, M., Wojci, K. S. M., Hassouna, I., et al. (2011). Expression of constitutively active erythropoietin receptor in pyramidal neurons of cortex and hippocampus boosts higher cognitive functions in mice. BMC Biol. 9:27. doi: 10.1186/1741-7007-9-27

Sargin, D., Friedrichs, H., El-Kordi, A., and Ehrenreich, H. (2010). Erythropoietin as neuroprotective and neuroregenerative treatment strategy: comprehensive overview of 12 years of preclinical research. Best Pract. Res. Clin. Anaesthesiol. 24, 573-594. doi: 10.1016/j.bpa.2010.10.005

Shein, N. A., Horowitz, M., Alexandrovich, A. G., Tsenter, J., and Shohami, E. (2005). Heat acclimation increases hypoxia-inducible factor 1alpha and erythropoietin receptor expression: implication for neuroprotection after closed head injury in mice. J. Cereb. Blood Flow Metab. 25, 1456-1465. doi: $10.1038 /$ sj.jcbfm. 9600142

Sinor, A. D., and Greenberg, D. A. (2000). Erythropoietin protects cultured cortical neurons, but not astroglia, from hypoxia and AMPA toxicity. Neurosci. Lett. 290, 213-215. doi: 10.1016/s0304-3940(00)01361-6

Sirén, A. L., Fratelli, M., Brines, M., Goemans, C., Casagrande, S., Lewczuk, P., et al. (2001). Erythropoietin prevents neuronal apoptosis after cerebral ischemia and metabolic stress. Proc. Natl. Acad. Sci. U S A 98, 4044-4049. doi: 10.1073/pnas. 051606598

Sun, B., and Salvaterra, P. M. (1995). Characterization of nervana, a Drosophila melanogaster neuron-specific glycoprotein antigen recognized by anti-horseradish peroxidase antibodies. J. Neurochem. 65, 434-443. doi: 10.1046/j.1471-4159.1995.65010434.x

Ulrich, J., Dao, V. A., Majumdar, U., Schmitt-Engel, C., Schwirz, J., Schultheis, D., et al. (2015). Large scale RNAi screen in Tribolium reveals novel target genes for pest control and the proteasome as prime target. BMC Genomics 16:674. doi: 10.1186/s12864-015-1880-y

Um, M., Gross, A. W., and Lodish, H. F. (2007). A "classical" homodimeric erythropoietin receptor is essential for the antiapoptotic effects of erythropoietin on differentiated neuroblastoma SH-SY5Y and pheochromocytoma PC-12 cells. Cell. Signal. 19, 634-645. doi: 10.1016/ j.cellsig.2006.08.014

Vanhems, E., and Delbos, M. (1987). Differentiation of glial cells and neurite outgrowth obtained from embryonic locust central nervous system explants. Brain Res. 411, 129-138. doi: 10.1016/0006-8993(87)90689-5

Weishaupt, J. H., Rohde, G., Pölking, E., Siren, A. L., Ehrenreich, H., and Bähr, M. (2004). Effect of erythropoietin on axotomy-induced apoptosis in rat retinal ganglion cells. Invest. Ophthalmol. Vis. Sci. 45, 1514-1522. doi: 10.1167/iovs. 03-1039

Wyder, S., Kriventseva, E. V., Schröder, R., Kadowaki, T., and Zdobnov, E. M. (2007). Quantification of ortholog losses in insects and vertebrates. Genome Biol. 8:R242. doi: 10.1186/gb-2007-8-11-r242

Yang, F., Xu, Y.-P., Li, J., Duan, S.-S., Fu, Y.-J., Zhang, Y., et al. (2009). Cloning and characterization of a novel intracellular protein $\mathrm{p} 48.2$ that negatively regulates cell cycle progression. Int. J. Biochem. Cell Biol. 41, 2240-2250. doi: 10.1016/ j.biocel.2009.04.022

Conflict of Interest Statement: The authors declare that the research was conducted in the absence of any commercial or financial relationships that could be construed as a potential conflict of interest.

Copyright (C) 2017 Hahn, Knorr, Liebig, Wüstefeld, Peters, Büscher, Bucher, Ehrenreich and Heinrich. This is an open-access article distributed under the terms of the Creative Commons Attribution License (CC BY). The use, distribution or reproduction in other forums is permitted, provided the original author(s) or licensor are credited and that the original publication in this journal is cited, in accordance with accepted academic practice. No use, distribution or reproduction is permitted which does not comply with these terms. 
Tc-crlf3 orthologue fragment 1 (638bp):

GGGTGATAGGAACGAAGTGGTGGAAACATTAAAAGCGGCAAAATCGTACCTAAATCACTTGG AAAATTTAAACAAGCAGCTGGAAAATGCGGAAAACCAAATTTGCGAAACTTCCAGCGAAACAG AGAGGAACATTGAAGTCACTTTTAGCAATCTTTTGACCAATCTGACGGAGATTTTATTAAATCG TAAGGATATTCTAATTAAAAAAGTACAAAAAACGAGGAAGCAAAGTTTGGCTCCTTTAGACGCA AGCAGATCTGATATTTTTAGTAACATTGAAAAGACCAAGAAACTGATAAAAGAGGGCGAATATC TGTTAAGTGGTAGTAGGGAAAACTTGGCTGAGTTTAGCCAAAATCAAGCTTCCTTGGAAGTC TTCCTGCAATTCCAGAGCTCAAAGAAGTGCCTTATATTTCATTCCACTGTGAACCAAATTTAGA GTCGGAATTGCTCGACATTTGCTCCCAGTTTGGCGAAGTTTCCAGAATTGCTCCAGTCCAAAT AAGCCAAACTGTTGAAAAACCTGGCGCAATTCTGGTCGAGTGGCAAAGTGTAGACGATGAGC GCTGTGTCGATATTCAAGAATTTCGTCTCCAGAGAGCATTTGGGGACGTCATATCTGAGAAAC ACTTAG

\section{Tc-crlf3 orthologue fragment 2 (527bp):}

CGATTGTTATGTGGGCGCAGAGACGCAGTTTTTAGTCAAGGATTTACGCCCCAACCAGTTGTA TTCTTTCAGAGTTTGTTGTAAATTTGAAGGCTCGAGTGACTGGAGTCCATGGTCTTTGTCCCAA GTCACTAGGACAAGCATCAAGCCATTTTCTTGGGCCAGTAATAGAGATTTTGTTTTTGCTGATG ACAATAAAATTGCCACACCTGTGAAAGATGCGCCGAGTTTATTATTGTCTGATGGACCACAATT TGCCGTTGGATATTCTGTTGAGTTTTCGTATTTGGAAATTGATAATAAATGTTCTACGATTGCAT TAATTGCGGTCAAGGAAGCTGCTGGGATTAAGGAAATAAGGTTAGGCGATCACAGCTATTTTG CTCTTGATTCGACTGGGGAAATTTACGTTGATGGTGTAAAGAAGGCTACCAAGTTACCTGAAG CTGCCAAAGGATTCAAAATTTGCTTTATGTGTGAATTAATCCAAGATGACAAAGTACGAGTCAG TATTGATACGTGTAACA

\section{Fragment dsRed (427bp):}

CATGGTGCGCTCCTCCAAGAACGTCATCAAGGAGTTCATGCGCTTCAAGGTGCGCATGGAGG GCACCGTGAACGGCCACGAGTTCGAGATCGAGGGCGAGGGCGAGGGCCGCCCCTACGAGG GCCACAACACCGTGAAGCTGAAGGTGACCAAGGGCGGCCCCCTGCCCTTCGCCTGGGACAT CCTGTCCCCCCAGTTCCAGTACGGCTCCAAGGTGTACGTGAAGCACCCCGCCGACATCCCCG ACTACAAGAAGCTGTCCTTCCCCGAGGGCTTCAAGTGGGAGCGCGTGATGAACTTCGAGGAC GGCGGCGTGGTGACCGTGACCCAGGACTCCTCCCTGCAGGACGGCTGCTTCATCTACAAGGT GAAGTTCATCGGCGTGAACTTCCCCTCCGACGGCCCCGTAATGCAGAAGAAGACCATG

\section{Fragment Rpt3 (300bp):}

GACGAGCAACGCAACTTGAAGAAGGAGTATCTCCACGCCCAGGAGGAGGTGAAGCGGATCCA GTCGGTGCCGCTTGTCATCGGCCAGTTTCTGGAAGCCGTTGACCAAAATACTGGGATTGTGGG GTCAACCACCGGGTCGAATTATTACGTCCGGATTTTGTCAACGATTGACCGGGAATTGCTCAAA CCTTCGGCCAGTGTGGCTTTGCATAAGCACAGTAACGCCCTTGTGGACGTCCTCCCGCCTGAA GCTGATTCCTCGATCAGCATGCTCCAGGCTGATGAGAAGCCTGACGTC

Supplementary Figure S1: 5' to 3' strand of double stranded RNA (dsRNA) fragments used for RNAi in primary brain cell cultures from $T$. castaneum. 


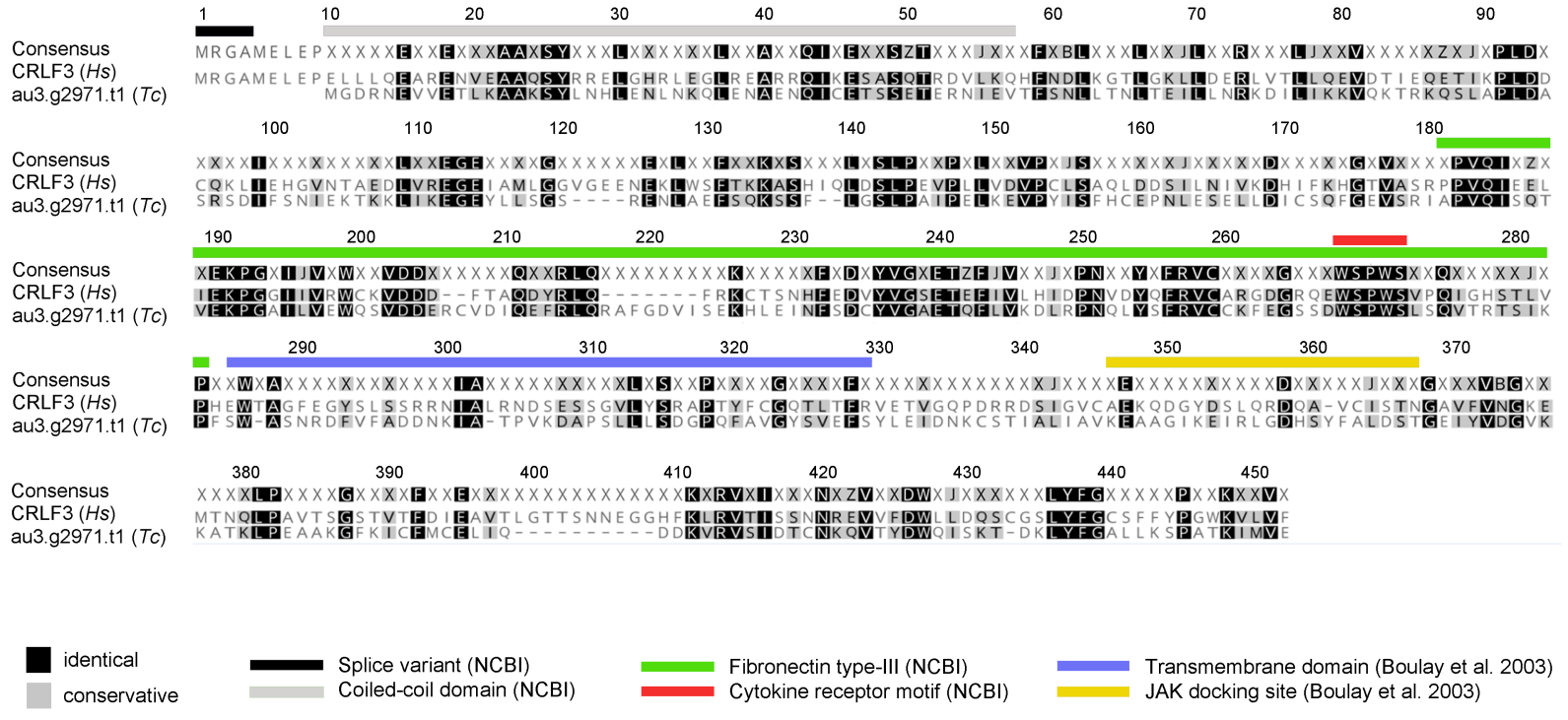

Supplementary Figure S2: Aligned amino acid sequences of human cytokine receptor-like factor 3 (CRLF3; NCBI GenBank NP_057070.3) and orthologous au3.g2971.t1-derived protein Tc-CRLF3 from T. castaneum. Predicted domains are indicated by bars on top of the sequence. Domain information related to human CRLF3 derived from NCBI (http:// www.ncbi.nlm.nih.gov/protein/Q8IUI8.2) and (24). 


\subsection{Manuscript 2: The Orphan Cytokine Receptor CRLF3 Emerged with the Origin of the Nervous System and Is a Neuroprotective Erythropoietin Receptor in Locusts}

The manuscript "The Orphan Cytokine Receptor CRLF3 Emerged with the Origin of the Nervous System and Is a Neuroprotective Erythropoietin Receptor in Locusts" was published in "Frontiers in Molecular Neuroscience" on $11^{\text {th }}$ October 2019.

Authors: Nina Hahn, Luca Büschgens, Nicola Schwedhelm-Domeyer, Sarah Bank, Bart R.H. Geurten, Pia Neugebauer, Bita Massih, Martin C. Göpfert and Ralf Heinrich.

This manuscript expands the knowledge about CRLF3 orthologs presented in manuscript 1 and provides an in-depth phylogenetic analysis. Overall, 293 CRLF3 highly conserved sequences were identified in metazoan species which emerged with the origin of the eumetazoan nervous system. In the insect Locusta migratoria, we detected the ortholog Lm-crlf3 and demonstrated its expression not only in the nervous system but also in hemolymph and to lower extend in skeletal muscle. This is in line with reports of $c r l f 3$ expression in various vertebrate tissues. It suggests that CRLF3 was originally involved in a protective function within the eumetazoan nervous system and has been later coopted for functions in other tissues. This manuscript furthermore shows that the insect ortholog Lm-CRLF3 is crucial for Epo-mediated neuroprotection in locust primary brain neurons as previously described for Tribolium. Moreover, soaking RNAi is also applicable to locust primary brain cell cultures. While Tribolium is a holometabolous insect, Locusta is hemimetabolous. The results demonstrate high conservation of CRLF3 and substantiate the hypothesis that CRLF3 might be a neuroprotective Epo-inducible receptor in vertebrates being an interesting target for neuroprotective therapies.

I substantially contributed to this manuscript. I collected experimental data together with LB, NSD, $\mathrm{BM}, \mathrm{PN}$ and $\mathrm{RH}$. In addition, I performed data analysis and interpretation. I prepared all figures and initiated the collaboration with SB for the phylogenetic analysis of CRLF3 sequences. Together with $\mathrm{RH}, \mathrm{I}$ designed and supervised the study. I drafted the manuscript and managed the submission as well as the revision as a corresponding author. 


\section{OPEN ACCESS}

Edited by:

Vsevolod V. Gurevich, Vanderbilt University, United States

Reviewed by:

Dietmar Benke,

University of Zurich, Switzerland

Philip Forsyth Copenhaver, Oregon Health \& Science University,

United States

*Correspondence:

Nina Hahn

nina.hahn@uni-goettingen.de

Ralf Heinrich

rheinri1@gwdg.de

Received: 13 August 2019 Accepted: 27 September 2019

Published: 11 October 2019

Citation:

Hahn N, Büschgens L,

Schwedhelm-Domeyer N, Bank S,

Geurten BRH, Neugebauer $P$, Massih B, Göpfert MC and Heinrich $R$ (2019) The Orphan Cytokine Receptor

CRLF3 Emerged With the Origin

of the Nervous System and Is

a Neuroprotective Erythropoietin

Receptor in Locusts.

Front. Mol. Neurosci. 12:251. doi: 10.3389/fnmol.2019.00251
The Orphan Cytokine Receptor CRLF3 Emerged With the Origin of the Nervous System and Is a Neuroprotective Erythropoietin Receptor in Locusts

\author{
Nina Hahn ${ }^{1 *}$, Luca Büschgens ${ }^{1}$, Nicola Schwedhelm-Domeyer ${ }^{1}$, Sarah Bank ${ }^{2}$, \\ Bart R. H. Geurten ${ }^{1}$, Pia Neugebauer ${ }^{1}$, Bita Massih ${ }^{1}$, Martin C. Göpfert ${ }^{1}$ and \\ Ralf Heinrich ${ }^{1 *}$

\begin{abstract}
1 Department of Cellular Neurobiology, Institute for Zoology and Anthropology, Georg-August University of Göttingen, Göttingen, Germany, ${ }^{2}$ Department of Animal Evolution and Biodiversity, Institute for Zoology \& Anthropology, Georg-August University of Göttingen, Göttingen, Germany
\end{abstract}

The orphan cytokine receptor-like factor 3 (CRLF3) was identified as a neuroprotective erythropoietin receptor in locust neurons and emerged with the evolution of the eumetazoan nervous system. Human CRLF3 belongs to class I helical cytokine receptors that mediate pleiotropic cellular reactions to injury and diverse physiological challenges. It is expressed in various tissues including the central nervous system but its ligand remains unidentified. A CRLF3 ortholog in the holometabolous beetle Tribolium castaneum was recently shown to induce anti-apoptotic mechanisms upon stimulation with human recombinant erythropoietin. To test the hypothesis that CRLF3 represents an ancient cell-protective receptor for erythropoietin-like cytokines, we investigated its presence across metazoan species. Furthermore, we examined CRLF3 expression and function in the hemimetabolous insect Locusta migratoria. Phylogenetic analysis of CRLF3 sequences indicated that CRLF3 is absent in Porifera, Placozoa and Ctenophora, all lacking the traditional nervous system. However, it is present in all major eumetazoan groups ranging from cnidarians over protostomians to mammals. The CRLF3 sequence is highly conserved and abundant amongst vertebrates. In contrast, relatively few invertebrates express CRLF3 and these sequences show greater variability, suggesting frequent loss due to low functional importance. In L. migratoria, we identified the transcript $L m$-crlf3 by RACE-PCR and detected its expression in locust brain, skeletal muscle and hemocytes. These findings correspond to the ubiquitous expression of crlf3 in mammalian tissues. We demonstrate that the sole addition of doublestranded RNA to the culture medium (called soaking RNA interference) specifically interferes with protein expression in locust primary brain cell cultures. This technique was used to knock down $L m$-crlf3 expression and to abolish its physiological function. We confirmed that recombinant human erythropoietin rescues locust brain neurons from hypoxia-induced apoptosis and showed that this neuroprotective effect is absent after knocking down $L m$-crlf3. Our results affirm the erythropoietin-induced neuroprotective 
function of CRLF3 in a second insect species from a different taxonomic group. They suggest that the phylogenetically conserved CRLF3 receptor may function as a cell protective receptor for erythropoietin or a structurally related cytokine also in other animals including vertebrate and mammalian species.

Keywords: neuroprotection, erythropoietin, cytokine receptor, Locusta migratoria, soaking RNA interference, nervous system, ancient receptor, orphan receptor

\section{INTRODUCTION}

The cytokine receptor-like factor 3 (CRLF3) is a largely uncharacterized orphan cytokine receptor with unknown function and endogenous ligand. The human crlf3 gene (NCBI Accession No. NM_015986.4, synonyms Creme9, Cytor4, p48.2, p48.6) is located on chromosome 17 and spans 2873 base pairs. The human CRLF3 spans 442 amino acids comprising the conserved cytokine receptor motif WSXWS, a single transmembrane segment and an intracellular Janus kinase (JAK) docking site. These characteristics identify CRLF3 as a member of the group 1 in the prototypic class I cytokine receptors that typically bind class 1 helical cytokines (Boulay et al., 2003; Liongue and Ward, 2007). Group 1 also contains the classical erythropoietin receptor (EpoR), the thrombopoietin receptor, the prolactin receptor and the growth hormone receptor. They typically function as homo- or hetero-dimers or associate to multimeric receptor complexes (Boulay et al., 2003). CRLF3 is expressed in various human tissues, including pancreas, kidney, and brain amongst others (Yang et al., 2009). In addition, freshly isolated tumor tissues and some tumor cell lines show elevated CRLF3 expression (Dang et al., 2006; Yang et al., 2009). CRLF3 has been associated with signal transducer and activator of transcription 3 (STAT3) activation, cell cycle regulation, neuronal morphology, and amyotrophic lateral sclerosis (Yang et al., 2009; Hashimoto et al., 2012; Cirulli et al., 2015). However, its physiological function is yet to be determined. Given its structural similarities to EpoR, we have investigated the potential involvement of CRLF3 in erythropoietin (Epo)-mediated neuroprotection.

Even though epo and $e p o R$ are not present in invertebrate genomes, previous in vitro and in vivo studies demonstrated neuroprotective and neuroregenerative effects of recombinant human erythropoietin (rhEpo) in the insects Locusta migratoria and Tribolium castaneum (Ostrowski et al., 2011; Miljus et al., 2014; Hahn et al., 2017). We found several parallels between Epomediated neuroprotection in mammals and insects including activation of JAK/STAT intracellular signaling, induction of antiapoptotic proteins, initiation of receptor endocytosis after Epobinding, and sensitivity to the non-erythropoietic human Epo splice variant EV-3 (Miljus et al., 2014, 2017; Hahn et al., 2017; Heinrich et al., 2017). These findings supported the hypothesis (Brines and Cerami, 2005; Ostrowski et al., 2011; Ghezzi and Conklin, 2013) that Epo signaling originally functioned as an adaptation mechanism to challenging physiological conditions (e.g., infections, metabolic stress, injury, hypoxia) and only later evolved to regulate vertebrate red blood cell production (summarized by Jelkmann, 2011). Since cytokines commonly activate different receptors and cytokine receptors often respond to several cytokine ligands, we explored the hypothesis that CRLF3 serves as the neuroprotective receptor stimulated by rhEpo in insects.

We previously demonstrated that CRLF3 is crucial for Epomediated neuroprotection in hypoxia-exposed neurons from the beetle T. castaneum (Hahn et al., 2017). In contrast, Epo showed no cell protective effects in in vitro studies using macrophagelike Schneider (S2) cells and neuron-like BG2-c2 cells derived from the fruit fly Drosophila melanogaster (unpublished data). Drosophila lacks a crlf3 gene (Wyder et al., 2007; Hahn et al., 2017, this study) supporting the hypothesis that CRLF3 may function as a neuroprotective receptor for Epo. Investigating CRLF3 as a neuroprotective Epo receptor contributes to the understanding of Epo as a neuroprotective agent and may support the development of alternative, safe treatments for neurological and neurodegenerative diseases that, unlike Epo itself, do not stimulate adverse side effects (Leist et al., 2004; Unger et al., 2010).

To the present, studies on CRLF3 involvement in Epomediated cell protection have only been performed in the beetle T. castaneum (Coleoptera). Here, we study locust primary brain cells (L. migratoria, Orthoptera) to confirm the hypothesis that CRLF3 represents an evolutionary ancient cell protective receptor. In vivo cellular functions can best be modeled in vitro by primary cell cultures, since their cellular development took place in natural environment. In vitro loss of function studies with mammalian cells require electroporation, lipid-mediated or viral-mediated transfection in order to induce gene targeted RNA interference (RNAi) and are prone to low efficiency. Locust primary brain neurons have the advantage that they spontaneously take up double-stranded RNA from the medium. This initiates the RNAi machinery, specifically suppressing the production of a protein of interest (called soaking RNAi). We applied soaking RNAi for a loss of function study in order to investigate the function of Lm-CRLF3 in primary locust brain cells.

The present study provides further evidence for the importance of CRLF3 in Epo-mediated neuroprotection using locust neurons. Moreover, we show Lm-crlf3 expression in a variety of locust tissues, arguing for a general cell protective function of CRLF3. Phylogenetic analysis resulted in 293 eumetazoan species expressing CRLF3 with the earliest appearance in the last common ancestor of Cnidaria and Bilateria. This indicates that its original function might have been related to the eumetazoan nervous system. Later in evolution, CRLF3 was coopted for functions also in other tissues leading to frequent expression and high sequence conservation amongst 
vertebrate species. We furthermore validate soaking RNAi in locust neurons as an appropriate technique for robust loss of function studies in vitro.

\section{MATERIALS AND METHODS}

\section{Primers}

TABLE 1 | Summary of oligonucleotides.

\begin{tabular}{|c|c|}
\hline Name & DNA sequence $\left(5^{\prime}->3^{\prime}\right)$ \\
\hline UPM long T3 & $\begin{array}{l}\text { 5' ATTAACCCTCACTAAAGGGAAA } \\
\text { GCAGTGGTATCAACGCAGAGT 3' }\end{array}$ \\
\hline UPM short T3 & 5' ATTAACCCTCACTAAAGGGA 3' \\
\hline RACE_Lm-crlf3_for & 5' GGTTCATGCTGTTGAGAGGGTTGGCAG 3' \\
\hline RACE_Lm-crlf3_rev & 5' CTGCCAACCCTCTCAACAGCATGAACC 3' \\
\hline Lm-crlf3 F1 Fw & 5' GTGTGATAGGTTGCCAGCAGTC 3’ \\
\hline Lm-crlf3 F1 Rv & 5' CGTATAAGGTGGTGACATTCAGGTC 3' \\
\hline Lm-crlf3 F2 Fw & 5' GGAACCAGTCACTCTGCGAG 3' \\
\hline Lm-crlf3 F2 Rv & 5' CGAATATTACCCCAGGCTGGAG 3' \\
\hline Lm-rpt3 full Fw & 5' TTGGGGATCGGTGCGTCAG 3' \\
\hline Lm-rpt3 full rV & 5' TTATTTATAGAATTCATGCTCTGATTCATCC 3' \\
\hline Lm-rpt3 Fw & 5' GATGAGCAGCGCAATITGAAAA 3' \\
\hline Lm-rpt3 Rv & 5' CACATCTGGCTIITCATCTGC 3' \\
\hline $\mathrm{qLm}$-gapdh Fw & 5' GTCTGATGACAACAGTGCAT 3' \\
\hline $\mathrm{q} L m$-gapdh $\mathrm{Rv}$ & 5' GTCCATCACGCCACAACTाTC 3' \\
\hline $\mathrm{q} L m-C R L F 3 \mathrm{FW}$ & 5' GTCTGGCTCTTGCCGATCACC 3' \\
\hline $\mathrm{q} L m-C R L F 3 \mathrm{Rv}$ & 5' GTAGTCTTCCCTTGCCATCCACAAACACAC 3' \\
\hline M13F & 5' GTAAAACGACGGCCAGT 3' \\
\hline M13R-T7 & 5' taatacgactcactataggCAGGAAACAGCTATGAC 3’ \\
\hline
\end{tabular}

\section{Plasmids}

The plasmid pDsRed (GB0100) was a gift from Diego Orzaez (Addgene plasmid \# 68202 ${ }^{1}$; RRID:Addgene_68202) (Sarrion-Perdigones et al., 2013). Lm-crlf3 fragment 1 and fragment 2 were designed as two non-overlapping fragments. They were inserted into the pCRII vector (TA Cloning ${ }^{\circledR}$ Kit Dual Promoter with $\mathrm{pCR}^{\mathrm{TM}} \mathrm{II}$ vector, Thermo Fisher Scientific, Germany) by TA-cloning, respectively. Then, pCRII_Lm-crlf3_F1 and pCRII_Lm-crlf3_F2 plasmids were transformed into XL1-Blue competent cells (\#200249, Agilent, United States) and purified with the NucleoBond ${ }^{\circledR}$ Xtra Midi kit (Macherey-Nagel, Germany) according to the user manual. The plasmid DNA was eluted in $500 \mu \mathrm{l} \mathrm{H}_{2} \mathrm{O}$. Lm-rpt3 was identified using BLAST and the LocustBase official gene set (OGS CDS V2.4.1) ${ }^{2}$ (Altschul et al., 1990). The sequence LOCMI02241 was determined as Lm-rpt3: the full-length CDS (submitted to GenBank with Accession No. MN245517) and a fragment of Lm-rpt3 were cloned into the pCRII vector as described above. All sequences are summarized in Supplementary File S1.

\footnotetext{
${ }^{1}$ http://n2t.net/addgene:68202

${ }^{2}$ http://www.locustmine.org/viroblast/viroblast.php
}

\section{Animals}

Locusts (L. migratoria) were purchased from Feeders \& more (Au i.d. Hallertau, Germany) and HW-Terra (Herzogenaurach, Germany). They were kept in groups at $24^{\circ} \mathrm{C}, 55 \%$ air humidity and $12 \mathrm{~h} / 12 \mathrm{~h}$ dark/light cycle for up to 1 week. Food was composed of organic lettuce leafs and reed ad libitum. Since this study was conducted exclusively with insects, it does not require a special permission. All experiments comply with the German laws for animal welfare ("Deutsches Tierschutzgesetz").

\section{Phylogenetic Analysis}

We searched for CRLF3 sequences with the blastp algorithm and default settings using the Geneious ${ }^{\circledR}$ 11.1.5 (Biomatters, Ltd.) BLAST tool and the human CRLF3 sequence (Q8IUI8.2) as query sequence. The NCBI accession numbers of the resulting hits are listed in Supplementary File S2. The CRLF3 sequence of L. migratoria was obtained by RACE PCR (rapid amplification of cDNA-ends with polymerase chain reaction) and submitted to GenBank (Accession No. MN245516). The CRLF3 sequence of Gryllus bimaculatus was obtained by using the tblastn search on the ASGARD data base ${ }^{3}$. The resulting hit GB-isotig00932 was translated into all possible reading frames. We used the translated sequence of reverse frame 3 since it comprises the CRLF3 characteristic motif WSXWS and an appropriate stop codon. All amino acid sequences were aligned using ClustalW version 2.1 implemented in Geneious ${ }^{\circledR}$ with default settings. Subsequently, we removed all columns that consisted of more than $50 \%$ missing data from the alignment resulting in a length of 438 amino acids. The phylogenetic tree was inferred with IQ-TREE version 1.6.8 (Nguyen et al., 2015) using the suggested substitution model JTT + R6 (Wong et al., 2017). Support values were computed using the implemented ultrafast bootstrap approximation and 1000 replicates (Minh et al., 2013; Hoang et al., 2018). The tree was rooted with the Cnidaria cluster.

\section{First Strand cDNA Synthesis and RACE PCR}

First-strand cDNA was synthesized from $1 \mu \mathrm{g}$ total RNA of brain tissue using SMARTer ${ }^{\circledR}$ RACE $5^{\prime} / 3^{\prime}$ Kit (Clontech, Takara, France) according to the user manual. Subsequently $5^{\prime}$ and $3^{\prime}$-rapid amplification of cDNA ends ( $5^{\prime}$ and $3^{\prime}$ RACE) was performed. The respective primers are summarized in Table 1. Gene-specific primers were designed on the partial sequences available at LocustBase ${ }^{2}$ and $i 5 \mathrm{k}^{4}$. The RACE PCR was performed with the following touchdown program and Advantage $^{\circledast} 2$ Polymerase Mix (Takara, France): initial step at $94^{\circ} \mathrm{C}$ for $2 \mathrm{~min}, 5$ cycles at $94^{\circ} \mathrm{C}$ for $30 \mathrm{~s}$ and $72^{\circ} \mathrm{C}$ for $5 \mathrm{~min}, 10$ cycles at $94^{\circ} \mathrm{C}$ for $30 \mathrm{~s}, 70^{\circ} \mathrm{C}$ for $30 \mathrm{~s}$ and $72^{\circ} \mathrm{C}$ for $5 \mathrm{~min}, 25$ cycles at $94^{\circ} \mathrm{C}$ for $30 \mathrm{~s}, 68^{\circ} \mathrm{C}$ for $30 \mathrm{~s}$ and $72^{\circ} \mathrm{C}$ for $5 \mathrm{~min}$, and a final step at $72^{\circ} \mathrm{C}$ for $5 \mathrm{~min}$. PCR products were analyzed by $1 \%$ agarose gel electrophoresis and purified with the NucleoSpin ${ }^{\circledR}$ Gel and PCR Clean-up kit (\#740609.50, Macherey-Nagel, Germany). Afterwards, they were cloned into

\footnotetext{
${ }^{3}$ http://asgard.rc.fas.harvard.edu/blast.html

${ }^{4}$ https://i5k.nal.usda.gov/locusta-migratoria
} 
the pCRII vector (\#K207040, TA Cloning ${ }^{\circledR}$ Kit Dual Promoter with $\mathrm{pCR}^{\mathrm{TM}} \mathrm{II}$ vector, Thermo Fisher Scientific, Germany), transformed into XL1-Blue competent cells (\#200249, Agilent, United States), purified and sequenced with M13 primers. The obtained full length mRNA sequence of $L m$-crlf3 was submitted to GenBank (Accession No. MN245516) and used for gene specific primer design.

\section{Dissection of Locust Tissue and RNA Isolation}

2-4 adult or 4 juvenile locusts were used for total RNA extraction. Brain, muscle and hemocytes RNA was isolated using the ZR Tissue \& Insect RNA MicroPrep ${ }^{\text {TM }}$ Kit (\#R2030, Zymo Research). Hemolymph (final amount $1.5 \mathrm{ml}$ ) was collected by injecting $500 \mu \mathrm{l}$ anticoagulant solution (98 $\mathrm{mM} \mathrm{NaOH}$, $186 \mathrm{mM} \mathrm{NaCl}, 17 \mathrm{mM} \mathrm{Na} 2$ EDTA, $41 \mathrm{mM}$ citric acid, pH 4.5) into the abdomen of a locust. After $1 \mathrm{~min}$, the hemolymph was collected with a pipette through an abdominal incision and stored on ice until further usage. Hemocytes were spun down, resuspended in $800 \mu \mathrm{l}$ RNA lysis buffer and transferred to a ZR BashingBead ${ }^{\mathrm{TM}}$ Lysis Tube. Brains were dissected as described in Miljus et al. (2014). Skeletal muscle originated from 4 to 6 large wing muscle strands. Tissue from either brain or muscle was directly collected in $800 \mu \mathrm{l}$ RNA lysis buffer in a ZR BashingBead ${ }^{\mathrm{TM}}$ Lysis Tube on ice. The following steps were performed according to the user manual including the on-column DNAse I treatment.

Total RNA from primary brain cell cultures was purified using the Monarch ${ }^{\circledR}$ Total RNA Miniprep Kit (\#T2010S, New England BioLabs ${ }^{\circledR} \mathrm{GmbH}$, Germany) according to the user manual including the recommended on-column DNAse I treatment. Cells were mechanically detached from the coverslips and directly transferred into $300 \mu \mathrm{l}$ lysis buffer. Finally, RNA was eluted twice with $20 \mu \mathrm{l}$ nuclease free water and stored at $-80^{\circ} \mathrm{C}$.

\section{cDNA Synthesis, Reverse Transcription PCR (RT-PCR), and Quantitative Real-Time PCR (qRT-PCR)}

cDNA was synthesized from $1 \mu \mathrm{g}$ total RNA using QuantiTect Reverse Transcription Kit (\#205311, Qiagen, Germany) according to the user manual. RT-PCR was performed with $100 \mathrm{ng}$ cDNA template, $0.4 \mu \mathrm{M}$ forward and reverse primers targeting Lm-crlf3 F1 and GoTaq ${ }^{\circledR}$ Green Master Mix (Promega, Germany) in a $25 \mu \mathrm{l}$ reaction volume. The PCR program consisted of an initial denaturing step at $95^{\circ} \mathrm{C}$ for $3 \mathrm{~min}, 30$ cycles of $95^{\circ} \mathrm{C}$ for $30 \mathrm{~s}, 58^{\circ} \mathrm{C}$ for $30 \mathrm{~s}$ and $72^{\circ} \mathrm{C}$ for $45 \mathrm{~s}$ and a final step at $72^{\circ} \mathrm{C}$ for $3 \mathrm{~min}$. Amplicons were analyzed by $1 \%$ agarose gel electrophoresis. qRT-PCR was conducted with the MyiQ ${ }^{\mathrm{TM}}$ Single-ColorReal-Time PCRDetection System (\#170-9740, BioRad, Germany) in 96-well plates (\#HSS9665, Bio-Rad, Germany) covered with a seal (\#MSB1001, Bio-Rad, Germany). The final reaction volume was $10 \mu \mathrm{l}$ containing $5 \mu \mathrm{l}$ of iTaq $^{\mathrm{TM}}$ Universal SYBR $^{\circledR}$ Green Supermix (\#1725121, Bio-Rad, Germany), $0.1 \mu \mathrm{M}$ primers and $10 \mathrm{ng}$ cDNA. Primers were tested for efficiency and stability. Lm-gapdh was used as a reference gene (Van Hiel et al., 2009). Amplification was performed with this program: $95^{\circ} \mathrm{C}$ for
$3 \mathrm{~min}$ followed by 40 cycles of $95^{\circ} \mathrm{C}$ for $10 \mathrm{~s}, 60^{\circ} \mathrm{C}$ for $30 \mathrm{~s}$ and $72^{\circ} \mathrm{C}$ for $30 \mathrm{~s}$, and a final step at $95^{\circ} \mathrm{C}$ for $1 \mathrm{~min}$. Afterwards, melting curve analysis was performed starting at $55^{\circ} \mathrm{C}$ for $1 \mathrm{~min}$ and increasing the temperature in 81 cycles for $0.5^{\circ} \mathrm{C}$ every $10 \mathrm{~s}$ up to $95^{\circ} \mathrm{C}$. Data were analyzed by the comparative $\mathrm{C}_{\mathrm{T}}$ method (Livak and Schmittgen, 2001).

\section{Synthesis of Double-Stranded RNA (dsRNA)}

Template DNA was amplified by PCR using M13F and M13RT7 primers (M13R attached with an additional $\mathrm{T} 7$ promotor) using the following program: denaturation at $98^{\circ} \mathrm{C}$ for $3 \mathrm{~min}$, 30 cycles of $98^{\circ} \mathrm{C}$ for $30 \mathrm{~s}, 60^{\circ} \mathrm{C}$ for $30 \mathrm{~s}$ and $72^{\circ} \mathrm{C}$ for $30 \mathrm{~s}$, and a final step of $72^{\circ} \mathrm{C}$ for $5 \mathrm{~min}$. In vitro transcription of dsRNA was performed using a T7 transcription kit (MEGAscript ${ }^{\mathrm{TM}} \mathrm{T} 7$ Transcription Kit, Thermo Fisher Scientific, Germany) and 400600 ng template DNA. RNA was purified by lithium chloride precipitation and resuspended in injection buffer $(1.4 \mathrm{mM} \mathrm{NaCl}$, $\left.0.07 \mathrm{mM} \mathrm{Na} \mathrm{HPO}_{4}, 0.03 \mathrm{mM}, \mathrm{KH}_{2} \mathrm{PO}_{4}, 4 \mathrm{mM} \mathrm{KCl}\right)$. RNA strands were annealed using a thermocycler and the following program: $60^{\circ} \mathrm{C}$ for $20 \mathrm{~min}, 95^{\circ} \mathrm{C}$ for $5 \mathrm{~min}$, decrease to $20^{\circ} \mathrm{C}$ in steps of $0.1^{\circ} \mathrm{C} / \mathrm{s}$. Size and quality of the dsRNA was checked with $1 \%$ agarose gel electrophoresis. Prior to usage, dsRNA was filtered through a sterile filter by centrifugation (Millex ${ }^{\circledR}-\mathrm{HV}$ Syringe Filter Unit, $0.45 \mu \mathrm{m}$, \#SLHV004SL, Millipore, Germany).

\section{Locust Primary Brain Cell Cultures}

Locust primary brain cell cultures were established from $4^{\text {th }}$ stage juvenile locusts as previously described (Ostrowski et al., 2011; Miljus et al., 2014). Complete growth medium consisted of L15 (Leibovitz's L-15 Medium, \#11415049, Thermo Fisher Scientific, Germany), 5\% FBSG (Fetal Bovine Serum Gold, PAA Laboratories GmbH, Austria), 1x Penicillin-Streptomycin (Penicillin-Streptomycin, 10,000 units penicillin and $10 \mathrm{mg}$ streptomycin/ml, \#P4333, Sigma-Aldrich ${ }^{\circledR}$, Germany) and 1\% Amphotericin B (Gibco ${ }^{\mathrm{TM}}$ Amphotericin B, $250 \mu \mathrm{g} / \mathrm{ml}$, \#15290018, Thermo Fisher Scientific, Germany). Dissected brains were pooled (see below), enzymatically digested with $2 \mathrm{mg} / \mathrm{ml}$ Collagenase/Dispase solution for $30-45 \mathrm{~min}$ at $27^{\circ} \mathrm{C}$ and mechanically dissociated by trituration with a $100 \mu \mathrm{l}$ tip of an Eppendorf pipette. The primary brain cells were cultured on ConcanavalinA-coated (Sigma-Aldrich ${ }^{\circledR}$, Germany) round glass cover slips $\left(\varnothing 10 \mathrm{~mm}\right.$, Corning, Inc., Sigma-Aldrich ${ }^{\circledR}$, Germany) in 4-well NUNC plates (\#176740, NuncTM Delta Surface, Thermo Fisher Scientific, Germany) filled with $500 \mu \mathrm{l}$ of complete growth medium at $27^{\circ} \mathrm{C}$ in a humidified atmosphere. The medium was changed every 2 days. Based on previous studies (Gocht et al., 2009), locust brain cultures are estimated to contain approximately 3\% glia and 97\% neurons after 7 days in vitro under normoxic conditions.

\section{Soaking RNAi}

Soaking RNAi describes the supplementation of standard growth medium with dsRNA to initiate a target-specific degradation of the respective transcripts. In order to investigate the applicability of soaking RNAi in locust primary brain cell cultures, we exposed 
cultures derived from the same pool of brain cells to dsRNA (final concentration $10 \mathrm{ng} / \mu \mathrm{l}$ ) targeting various transcripts. Fresh dsRNA was added with every medium change. Cells were fixed on day 5 and stained in order to assess the effect on cell viability as described below. dsRNA targeting Lm-rpt3 and $d s R e d$ were tested in this study. $L m$-RPT3 is a proteosomal regulatory protein that is essential for cellular survival and served as a positive control for RNAi efficacy. dsRNA targeting $d s R e d$ served as a negative control as dsRed is not naturally expressed in L. migratoria. Additionally, dsRNA targeting Lm-crlf3 fragment 1 and Lm-crlf3 fragment 2 was applied to otherwise untreated cultures during neuroprotection assays (see below) in order to exclude effects on cellular survival of the CRLF3 knock-down itself.

\section{Neuroprotection Assay and Pharmacological Treatment}

Neuroprotection assays compared cellular survival in cultures exposed to normoxia, hypoxia and rhEpo with or without previous knock-down of $\mathrm{Lm}$-crlf3 expression. Each experiment compared differently treated cultures that derived from the same pool of locust brain cells (two brains per culture/treatment). One experiment consisted of one culture at normoxic conditions (control), a hypoxia-treated culture (challenged, reduction to $<90 \%$ cell survival), a hypoxia- and Epo-treated culture (positive control for neuroprotective effect) and a hypoxiaand Epo-treated culture that was previously subjected to RNAi-induced Lm-CRLF3 knock-down (experimental group) (Figure 5). In some experiments, potential effects of dsRNA targeting $L m$-crlf3 were also determined in normoxic conditions. Control cultures were always incubated with the same medium and the same temperature as experimental cultures. In order to knock down $L m$-CRLF3, growth medium was supplemented with $10 \mathrm{ng} / \mu \mathrm{l}$ dsRNA ( $L m$-crlf3 fragment 1 or fragment 2 ) from day 0 to day 7. After 4 days, complete growth medium was replaced by growth medium without serum. On day 5 , in vitro cultures were treated with $32 \mathrm{ng} / \mathrm{ml}(\stackrel{\wedge}{=} \mathrm{U} / \mathrm{ml})$ rhEpo (NeoRecormon, Roche, Welwyn Garden City, United Kingdom) $12 \mathrm{~h}$ prior to $36 \mathrm{~h}$ hypoxia exposure.

Hypoxia $\left(\mathrm{O}_{2}\right.$ level $\left.<0.5 \%\right)$ was maintained in a hypoxic chamber (Hypoxia Incubator Chamber, \#27310, STEMCELL, ${ }^{\text {TM }}$ Germany) flooded with nitrogen. After hypoxic treatment, the cells were fixed for $30 \mathrm{~min}$ with $4 \%$ paraformaldehyde and stained with DAPI (1:1000) without agitation as described elsewhere to assess cell viability (Miljus et al., 2014; Hahn et al., 2017). The evaluation of cell viability (at the time of fixation) was performed on the basis of the DAPI-labeled nuclear morphology (Gocht et al., 2009). Photographs were taken with a Spot CCD camera (Invisitron, Germany) mounted on an epifluorescence microscope (Zeiss Axioskop; 40x objective, Germany). Numbers of alive and dead nuclei were evaluated using Fiji (Version 1.52.i) as described elsewhere (Schindelin et al., 2012; Hahn et al., 2017). The portion of living cells was determined for each culture and normalized to the portion of living cells in the normoxic control culture (set to 1). The experimenter was blinded with respect to the identity of the cultures while cell viability evaluation.

\section{Statistical Analysis}

Data analysis and statistics were performed with $\mathrm{R}$ (version 3.6.0) using R Studio (version 1.2.1335) (RStudio Team, 2018; $\mathrm{R}$ Core Team, 2019). Boxplots depict the median, the upper and lower quartile, and whiskers represent 1.5 times the interquartile range and outliers. Black circles represent the data of individual experiments. Statistics were calculated using the pairwise permutation test included within the packages "coin" and "rcompanion" (Hothorn et al., 2006, 2008; Mangiafico, 2019). The false discovery rate was controlled using the BenjaminiHochberg procedure (Benjamini and Hochberg, 1995).

\section{RESULTS}

\section{Identification of $\mathbf{L m}$-crlf3}

The sequence of the full length $L m$-crlf3 transcript was obtained by RACE PCR from locust brain tissue. It comprises 2522 bp and includes a 253 bp $5^{\prime}$ UTR, a 1320 bp coding sequence (CDS) and 949 bp $3^{\prime}$ UTR (Figure 1). The CDS determined in Geneious ${ }^{\circledR}$ refers to the translation of frame 2 and codes for 439 amino acids (see Supplementary File S1). The Lm-crlf3 sequence was used to transcribe double-stranded RNA targeting two non-overlapping fragments for RNAi experiments, for qRT-PCR to detect crlf3 expression in locust tissues and for phylogenetic analysis.

\section{Lm-crlf3 Expression}

In mammals, crlf3 is expressed in various tissues including the nervous system, reproductive organs, bone marrow and immune system. For comparison, crlf3 expression was determined in brain tissue, skeletal muscle and hemolymph. As determined by RTPCR amplifying Lm-crlf3 fragment 1 (displayed in Figure 1), all three tissues expressed $\mathrm{Lm}$-crlf3 in detectable amounts in both adult and juvenile locusts (Figure 2A). In both developmental stages, hemolymph seemed to contain the most and muscle the least amount of crlf3 transcripts. These semi-quantitative crlf3 expression levels were confirmed by qRT-PCR analysis of adult locust tissues using gapdh as reference. With respect to brain crlf3 expression (normalized to 1) hemolymph contained 11.16 $( \pm 1.99$ STDV) fold crlf3 transcripts while muscle contained only 0.09 ( \pm 0.06 STDV) fold (Figure 2B).

\section{Gene Tree of CRLF3}

BLAST searches with the human CRLF3 query detected CRLF3 sequences with reliable e-values ranging from 0 to $6.43 \mathrm{E}$ 07. Coverage of the human query varied between 16\% ( $P a n$ troglodytes) and $100 \%$, with a median of $97.29 \%$. The minimum sequence length was 86 amino acids (aa) (Pan troglodytes, Mammalia), the maximum 625 aa (Daphnia magna, Crustacea), whereas the median sequence length was 438 aa.

CRLF3 was shown to be present in 293 eumetazoan species ranging from Cnidaria to Mammalia. No hits were found in the basal metazoan taxa Porifera, Placozoa, and Ctenophora. A collapsed version of the CRLF3-based gene tree is shown in Figure 3, while the detailed version depicting all included species is presented as Supplementary Figure S1 and 


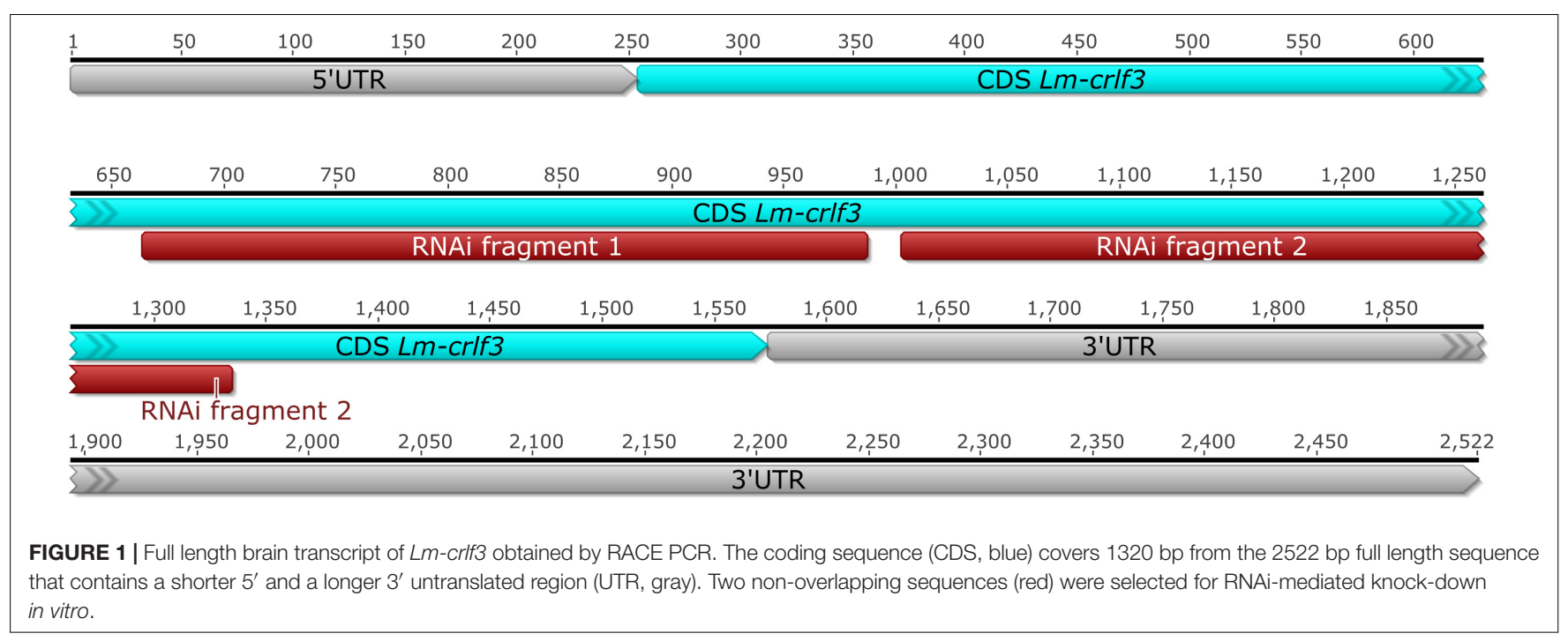
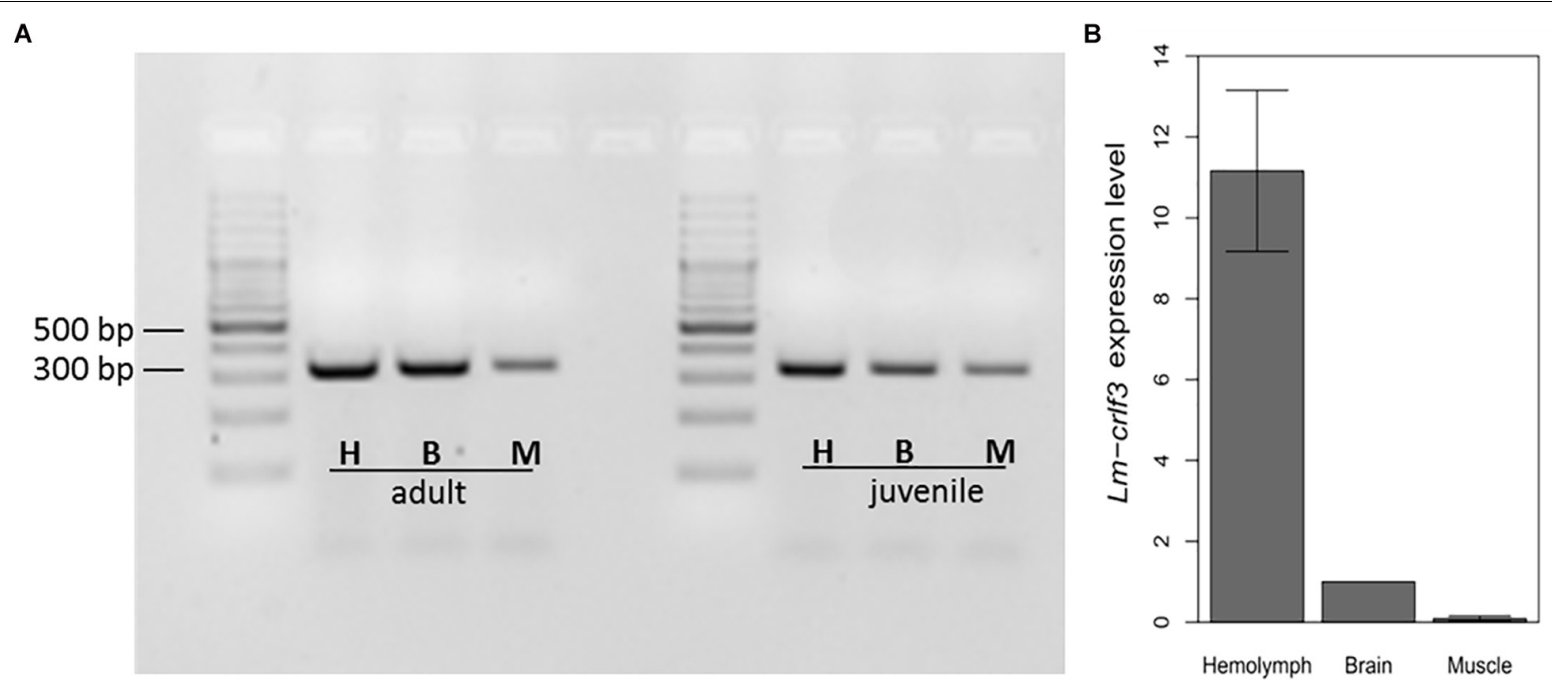

FIGURE 2 | Lm-crlf3 expression in hemocytes, brain and skeletal muscle. (A) $1 \%$ Agarose gel of RT-PCR products (using primers for $L m$-crlf3 fragment 1 and $100 \mathrm{ng}$ cDNA template) from hemocytes (H), brain (B), and skeletal muscle (M) extracted from adult and juvenile locusts. (B) qRT-PCR analysis of crlf3 expression in hemolymph, brain, and skeletal muscle of adult locusts. $L m$-gapdh was used as a reference gene and relative expression of $L m$-crlf3 was normalized to the level of expression in the brain (set to 1). Bars display average relative expression level \pm standard deviation. $N=3$.

Supplementary File S3. CRLF3 is present in only 34 invertebrate species while 259 hits were detected among vertebrate species. Branches are considerably longer in invertebrates than in vertebrates. Speciation events for major taxa are very wellsupported (>90\%).

\section{Soaking RNAi in Locust Primary Brain Cells}

We initially conducted control experiments to verify that cultured locust brain neurons take up dsRNA from the medium and initiate an RNAi response. We furthermore tested that soaking RNAi as such has no negative impact on cell survival. For these means, two dsRNA constructs (applied at $10 \mathrm{ng} / \mu \mathrm{l}$ concentration for 5 days) were evaluated in respect to their effect on neuronal cell survival. The first one targeted dsRed, a protein that is not naturally expressed in L. migratoria. As shown in Figure 4, dsRNA targeting dsRed had no significant effect on cell survival. In contrast, dsRNA targeting the expression of the proteasomal protein rpt3 caused a significant reduction of cellular survival $(p=0.0008$; median survival $74.8 \%$ compared to untreated controls from the same pool of cells). In addition, dsRNA targeting CRLF3 had no significant effect on cellular survival compared to untreated control cultures in both unchallenged and challenged cell cultures (Figure 5 and Supplementary Figure S3). These results indicate the functionality of soaking RNAi to suppress the translation of target proteins and serve as a control for the negligible impact of unspecific dsRNA and its solvent on cell viability. 


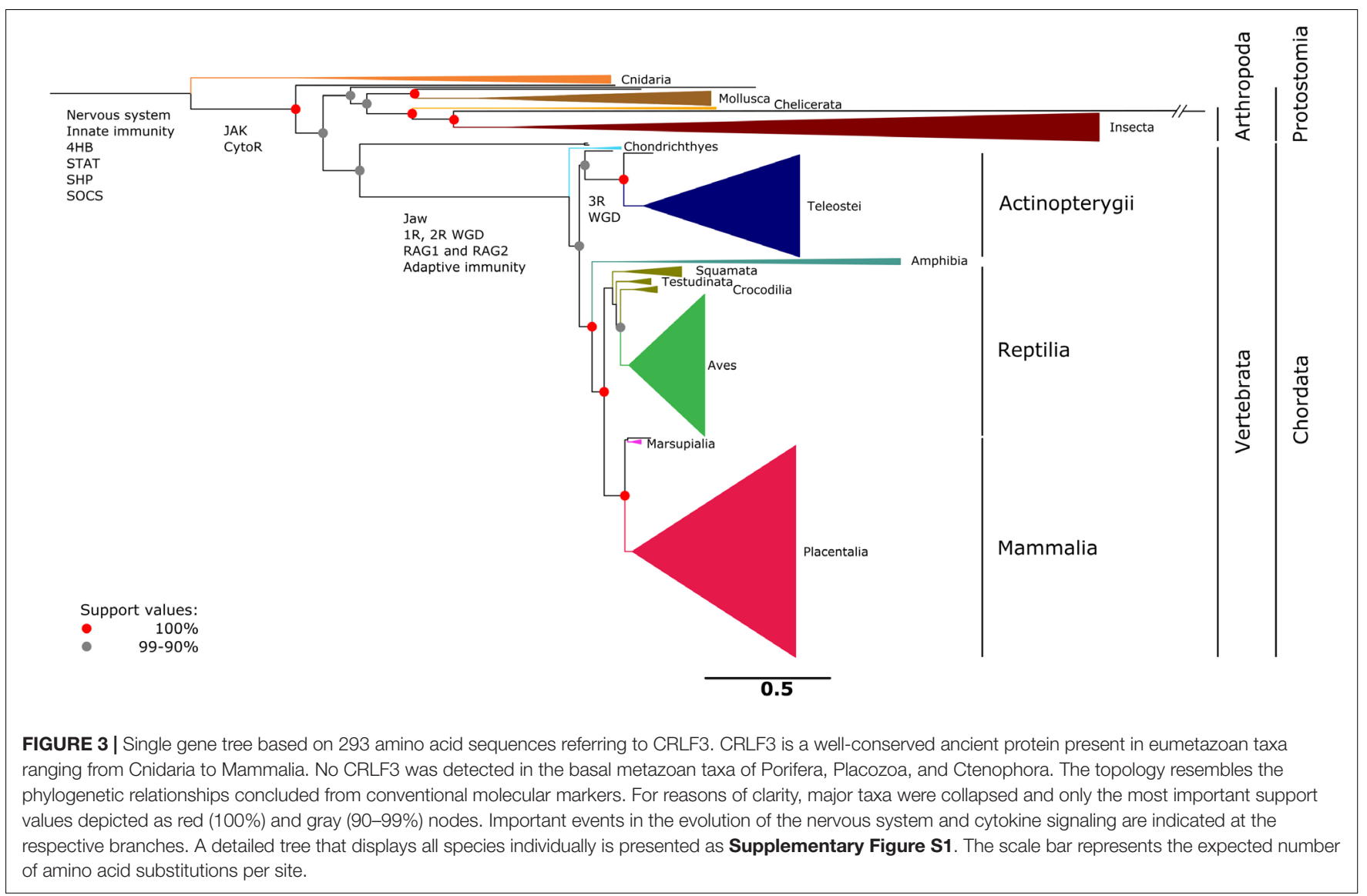

\section{Involvement of $L m$-CRLF3 in Epo-Mediated Neuroprotection}

After demonstrating a robust knock-down of $L m$-rpt3 following 5 days of exposure to dsRNA, we knocked down Lm-crlf3 expression to investigate its importance for Epo-mediated neuroprotection. In these experiments, we applied dsRNA with the same protocol (exposure to $10 \mathrm{ng} / \mu \mathrm{l}$ dsRNA for 5 days before experiments started) targeting two non-overlapping fragments of Lm-crlf3 to exclude off-target effects. qRT-PCR analysis proved that after 5 days of soaking RNAi the expression levels dropped by half compared to controls (Supplementary Figure S2). The involvement of CRLF3 in Epo-mediated neuroprotection of locust neurons was tested in a neuroprotection assay. Following identical protocols, two series of experiments were conducted, in which $L m$-CRLF3 expression was suppressed by two different dsRNA constructs (fragment 1 and fragment 2). The results of experiments with fragment 1 are shown in Figure 5 whereas the results of experiments with fragment 2 are displayed in Supplementary Figure S3. Cells were stressed by hypoxia $\left(<0.5 \% \mathrm{O}_{2}\right)$ and a normoxic group was used as control (cell viability was set to 1 ). Cell viability was significantly decreased by hypoxia, however, pre-treatment with $32 \mathrm{ng} / \mu \mathrm{l} \mathrm{rhEpo}$ protected cells from hypoxia-induced apoptosis. Knock-down of CRLF3 abolished Epo's neuroprotective effect and cell viability was not significantly different to sole hypoxia-treated cells. The phenotypes observed by soaking RNAi using dsRNA constructs targeting $L m$-crlf3 fragment 1 or fragment 2 are similar (compare Figure 5 and Supplementary Figure S3). Hence, unspecific offtarget effects resulting from interference with the expression of another protein are unlikely.

\section{DISCUSSION}

Lm-CRLF3 consists of 439 amino acids (aa) which is similar to the size of other insect CRLF3 sequences ranging from 391 to 504 aa. The size of all CRLF3 sequences included into the analysis varies between 86 and 625 aa. CRLF3 found in Gryllus bimaculatus, another orthopteran species, is the closest to LmCRLF3 (51\% identity). Similar to Lm-CRLF3, the T. castaneum CRLF3 receptor ( Tc-CRLF3) has previously been described as an Epo-responsive receptor involved in neuroprotection (Hahn et al., 2017). Both receptors share $35 \%$ similarity of their sequences while the similarity between the locust and the human sequence is $29 \%$.

\section{Phylogenetic Analysis of CRLF3 and Potential Functions}

CRLF3 sequences were identified in 293 species and included in the phylogenetic analysis. The single gene tree mirrors the phylogenetic relationships of major metazoan taxa concluded from studies using conventional molecular markers or 


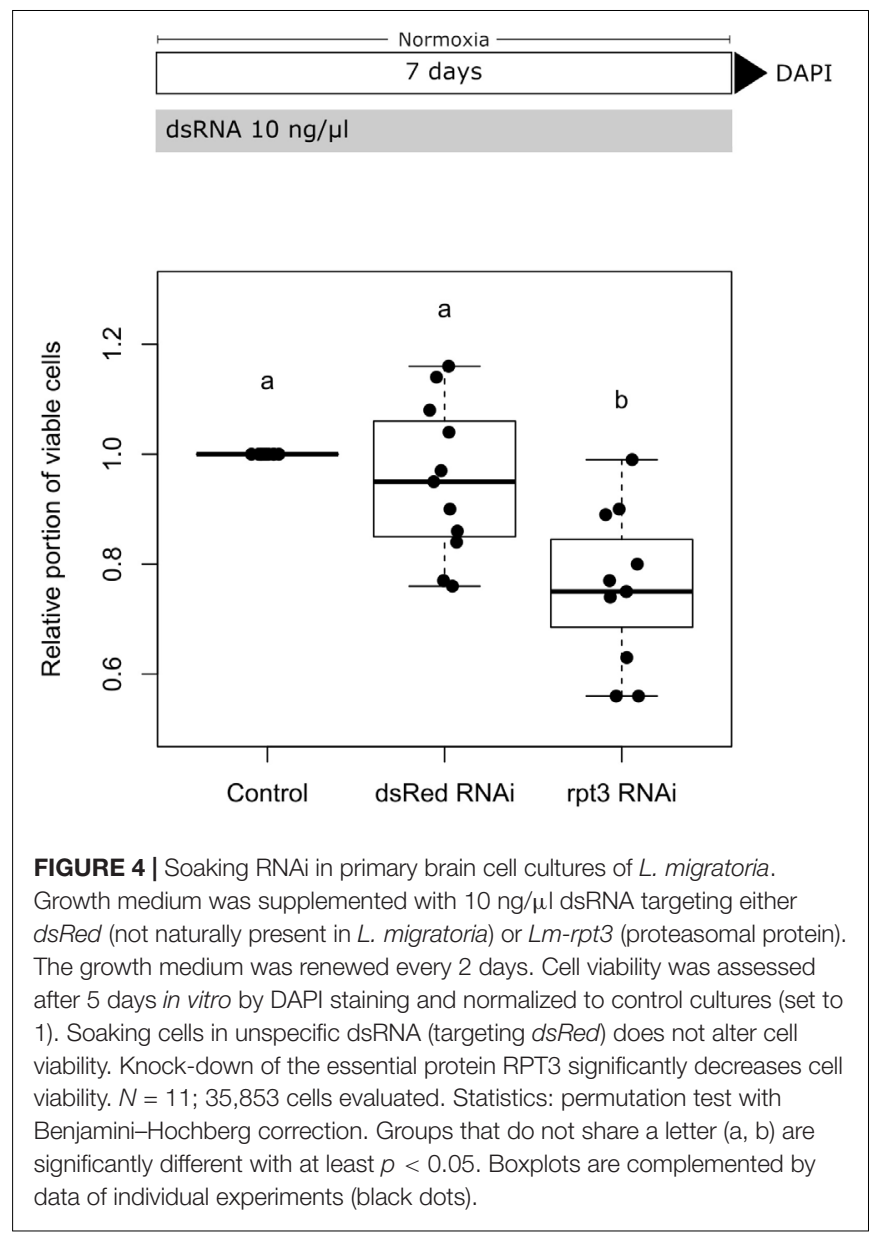

transcriptomes with remarkable detail (Dunn et al., 2014; Misof et al., 2014; Irie et al., 2018; Laumer et al., 2019). However, minor exceptions are present. The chordate Ciona intestinalis is represented as a sister group to all bilaterians but has been shown to be the sister group to vertebrates (Berná et al., 2009). The misplacement of Ciona might be explained by its particularly fast evolving genome (Berná et al., 2009). Furthermore, Latimeria chalumnae appears as sister to ray-finned fish (Actinopterygii). For decades, the phylogenetic position of Latimeria was subject to debate, but recent studies provide evidence for a closer relationship of Latimeria to tetrapods (Takezaki and Nishihara, 2016; Yoshida et al., 2019).

The high conservation of CRLF3 in vertebrates, reflected by short branches, suggests an important role for the organisms. Since the phylogenetic tree of CRLF3 resembles the molecular metazoan tree of life, CRLF3 seems to be subjected to rather high selective pressure leading to a fairly low evolutionary rate. The fact that no matches were found in Porifera, Placozoa and Ctenophora but in Cnidaria, suggests that CRLF3 might have evolved for some function in eumetazoan nervous systems (Grimmelikhuijzen et al., 1996; Westfall, 1996; Bosch et al., 2017). Porifera and Placozoa lack nervous systems. Nervous systems of Ctenophora are fundamentally different from eumetazoan counterparts (with respect to the presence of typical transmitters, gap-junctional proteins, expression of elav, large number of specific neuropeptides and others) (Moroz and Kohn, 2016). Thus, an independent evolutionary origin of ctenophoran and eumetazoan nervous systems is intensely debated (Jékely et al., 2015; Moroz and Kohn, 2016). Cnidaria possess extended neuronal networks that are regarded as homologous to the bilaterian nervous system. The presence of CRLF3 in Eumetazoa but not in Ctenophora matches the hypothesis that the nervous systems of both taxa have evolved convergently (Jékely et al., 2015; Moroz and Kohn, 2016). Main components of the CRLF3initiated signaling cascades were already present in ancestors of Cnidarians, including the four-helix-bundle (characteristic of class-I cytokines), SHP, STAT, and SOCS. These might have been complemented by JAK and CytoR in Bilateria forming the JAK/STAT signaling pathway (Babonis and Martindale, 2016; Liongue et al., 2016).

Although many invertebrate genomes (626 in NCBI) are available, CRLF3 is only sparsely present. Furthermore, the branch lengths amongst invertebrate taxa are comparably long indicating a lower conservation and higher diversification of this gene (Supplementary Figure S1). In order to exclude that the sparse representation of CRLF3 in insects is due to sequence unavailability and poor quality, we performed an additional analysis restricted to insects (data not shown). We analyzed the transcriptomes published by Misof and colleagues, who provided a robust phylogenetic tree based on high quality transcriptomes covering all extant insect orders and some other arthropods (144 in total) (Misof et al., 2014). Evaluation of this data set confirmed the low abundance of CRLF3 in insects (5 species out of 128). Hence, we hypothesize that CRLF3 has been lost in many invertebrates but was strictly maintained in vertebrates. Potential involvement in further physiological processes outside the nervous system, for instance within adaptive immunity, might have increased the selective pressure. In contrast to the innate immune system, which has already been present in early eukaryotes, the adaptive immune system, relying on $\mathrm{V}(\mathrm{D}) \mathrm{J}$ recombination, emerged in jawed vertebrates 450 million years ago (Rast et al., 1997; Dzik, 2010). It has been suggested that this adaptive immune system is an offshoot of the nervous system or that both derived from an ancestral neuro-immune cell (Bayne, 2003; Kioussis and Pachnis, 2009).

Both, the nervous and adaptive immune system share a variety of signaling molecules including neurotrophic factors, cytokines, chemokines (Habibi et al., 2009; Kerschensteiner et al., 2009; Ransohoff, 2009) and their receptors (Atwal et al., 2008; Levite, 2008; Ben Baruch-Morgenstern et al., 2014). Some molecules have initially been assigned as neurospecific and were later found to be involved in immune functions. For instance, the neuropeptide $\mathrm{Y}$ (orthologs are already present in insects) was first discovered as one of the most abundant neuropeptides in the central nervous system but has additional effects on immune cells (Brown et al., 1999; Wheway et al., 2007). In addition, the proteoglycan agrin was known to be required for the formation of the neuromuscular junction but is present on lymphocytes, too (Gautam et al., 1996; Khan et al., 2001; Zhang et al., 2006). In contrast, interleukin 2 was introduced as an immunoregulatory cytokine but more recent 


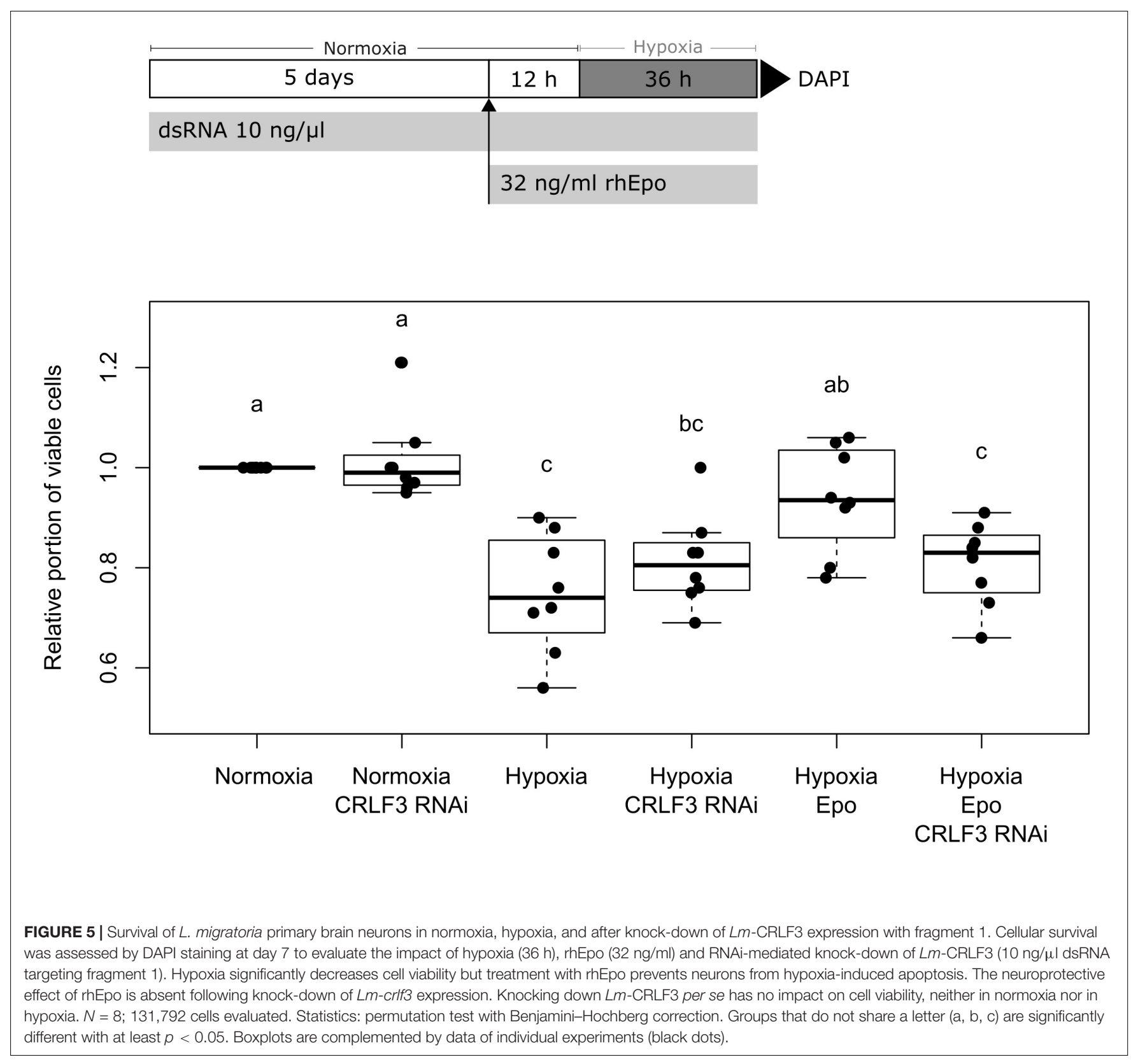

studies detected interleukin 2 production by neurons (Muraguchi et al., 1985; Meola et al., 2013). These shared chemical molecules involved in cell-to-cell communication support the hypothesis of an evolutionary common origin of the nervous and adaptive immune system.

Besides functional and physiological similarities, they also share morphological similarities. Both systems function via intimate associations, called synapses, at interfaces between homologous and heterologous cells. The term immunological synapse refers to the similarity to neural synapses and describes the contact between a $\mathrm{T}$ cell and an antigen presenting cell. The neural synapse and the immunological synapse share structural (e.g., adhesion molecules, cytokine secretion, receptor clustering) and functional (e.g., memory storage, exchange of information) commonalities (Donnadieu et al., 2001; Habibi et al., 2009). For instance, the proteoglycan agrin plays an important role in the construction and regulation of synapse formation in both synapse types (Gautam et al., 1996; Khan et al., 2001).

A major trigger for the development of this offshoot could have been two rounds of whole genome duplications (WGDs) that occurred after the split of invertebrates and the evolution toward jawed vertebrates (Gnathostomata) (Figure 3 and Supplementary Figure S1). According to the 2R hypothesis, the first WGD took place within early chordates and the second in the last common ancestor of gnathosthomes (Kasahara, 2007). WGDs facilitate the adaptation of genes and molecules to new functions and cooption into new tasks. After WGD, one copy becomes redundant lowering the selection pressure on the 
maintenance of its original function. This copy might either accumulate adverse mutations or gain beneficial modifications that enable the acquisition of new functions.

Furthermore, a horizontal gene transfer of a bacterial transposon after the second WGD might have led to the incorporation of the recombination activating genes (RAGs) RAG1 and RAG2 providing the basis for somatic $\mathrm{V}(\mathrm{D}) \mathrm{J}$ recombination within the adaptive immune system (Oettinger et al., 1990; Agrawal et al., 1998; Schatz, 2004; Kapitonov and Koonin, 2015). The V(D)J recombination occurs only in lymphocyte development generating the diverse repertoire of antigen receptors that are required for adaptive immunity (Oettinger et al., 1990). In this context, the orphan cytokine receptor might have been adapted to new functions of the adaptive immune system of jawed vertebrates. This hypothesis is supported by data indicating that WGDs diversified the functions of Tyrosine receptor kinases (Brunet et al., 2016). Furthermore, other class I cytokine receptors besides CRLF3 are also involved in the immune system (Holdsworth and Gan, 2015). The lack of this adaptive immunity in invertebrates might have altered the selective pressure on CRLF3. This hypothesis would explain the sparse occurrence as well as the high diversity seen in these species.

\section{Lm-crlf3 Is Expressed in Various Tissues}

In mammals, CRLF3 is expressed in a variety of tissues including the kidney, pancreas, and brain (Yang et al., 2009). Cell protective functions of Epo have also been reported in various tissues including the hematopoietic system, kidney, skeletal and heart muscle, pancreas, and others all of which also express CRLF3 (Brines and Cerami, 2006; Noguchi et al., 2008; Yang et al., 2009; Ogunshola and Bogdanova, 2013). This supports the hypothesis that CRLF3 might be a cell protective Epo receptor in mammals. In addition, similar expression patterns have been detected in various locust tissues including the nervous system, hemolymph, and skeletal muscle. Since hemocytes display adhesive properties, it cannot be excluded that low levels of crlf3 transcripts detected in wing muscle may result from contamination with adherent hemocytes that circulate throughout the hemocoel. This data suggests that CRLF3 is an ancient receptor that in the beginning had a function in general cell protective mechanisms and has been adapted to various tissues during evolution.

\section{Soaking RNAi for Loss of Function Studies in Locust Neurons}

We supplemented cell culture medium with dsRNA and let locust primary brain cells spontaneously take up dsRNA for 5 days. Medium and dsRNA were refreshed every 2 days to maintain a constant supply of dsRNA and nutrients. In order to assess the applicability of soaking RNAi in locust neuron cultures, we targeted the proteasomal protein RPT3 that is essential for cellular survival. The reduction of cellular survival to $75 \%$, compared to untreated control cultures, indicates that the neurons take up dsRNA and process it to small interfering RNA (siRNA) initiating mRNA degradation. dsRNA targeting dsRed, a protein that is not expressed in locusts, showed no significant reduction in cellular survival. By this we show that cell death was caused by the absence of RPT3 and not due to effects that were associated with dsRNA uptake. Moreover, dsRNA-mediated knock-down of Lm-crlf3 expression had no significant impact on neuronal survival, neither in normoxic nor hypoxic conditions. These results indicate that changes in cellular survival depended on the absence of specifically downregulated proteins [RPT3 and CRLF3 during hypoxia/Epo treatment (see below)] rather than on unspecific effects of dsRNA exposure.

RNAi in insects has been reported by various studies (reviewed in Vogel et al., 2019). Many of them focused on RNAi as a tool for pest control (Mamta and Rajam, 2017; Niu et al., 2018). However, the efficiency and success of RNAi varies tremendously between species and targeted tissues. Presumably, this depends on differences in dsRNA uptake and the ability to process dsRNA to siRNA (Ren et al., 2014; Wang et al., 2016; Singh et al., 2017). In vivo, dsRNA is typically delivered by feeding or injection into the hemocoel. It has been shown that dsRNAses are more abundant in the digestive system than in hemolymph. This is in line with observations showing that feeding dsRNA is often less effective than injecting dsRNA (Wang et al., 2016; Singh et al., 2017; Song et al., 2019). In locusts, feeding of dsRNA is not successful whereas injecting dsRNA leads to a robust systemic RNAi response (Luo et al., 2013; Song et al., 2019; Xie et al., 2019). The efficacy of RNAi differs between tissues. Injection of dsRNA into the hemocoel induces RNAi in the brain but not in ovaries (Ma et al., 2011; Ren et al., 2014). We herein introduce a new and convenient RNAi application method for loss of function studies in locust primary brain cell cultures. We termed it soaking RNAi since it requires no additional manipulations (e.g., lipofection, electroporation, viral delivery) to suppress specific protein expression as in mammalian cells. Soaking RNAi has been successfully applied in primary brain cell cultures from the beetle T. castaneum (Hahn et al., 2017). It has now been adapted to locust primary brain neurons, offering a new tool for in vitro loss of function studies in L. migratoria. The RNAi effect observed in L. migratoria is slightly lower than in the beetle T. castaneum (Hahn et al., 2017). Four days exposure of $T$. castaneum brain cells to $10 \mathrm{ng} / \mu \mathrm{l}$ dsRNA targeting rpt3 expression reduced cell survival to approximately $34-67 \%$ while 5 days exposure reduced $L$. migratoria median brain cell survival to approximately $75 \%$. In line with these observations, coleoptera, including $T$. castaneum, exhibit a generally higher RNAi susceptibility in comparison to L. migratoria (Wang et al., 2016; Singh et al., 2017). However, the RNAi response of L. migratoria is sufficiently robust and useful for loss of function studies.

\section{Lm-CRLF3 Is Crucial for Epo-Mediated Neuroprotection}

Selective neuroprotective activity (without stimulation of erythropoiesis) by EV-3 and other Epo-like ligands (e.g., carbamylated Epo, asialo-Epo, helix B surface peptide, Epo mimetic peptide 1) provided clear evidence for alternative cell protective Epo-receptors other than (EpoR) $)_{2}$ (Erbayraktar et al., 2003; Brines et al., 2004, 2008; Leist et al., 2004; Bonnas et al., 2017). Several receptors and receptor complexes have been 
associated with Epo-induced neuroprotection in mammals, including homodimeric $(\mathrm{EpoR})_{2}$, heteromeric EpoR/ $\beta$-common receptor and Ephrin B4 receptor (Brines et al., 2004; Um et al., 2007; Pradeep et al., 2016). However, Epo-mediated neuroprotection remains only partially understood. Hence, we investigated a potential neuroprotective involvement of CRLF3 in locust primary brain neurons.

Our experiments indicate that $L m$-CRLF3 represents an Epo-binding receptor, or alternatively constitutes an essential component of an Epo-binding receptor complex, whose activation can fully prevent hypoxia-induced apoptosis in locust primary brain cell cultures. RNAi against Lm-crlf3 does not generally affect the cell viability of locust primary brain neurons, neither in unchallenged nor in challenged conditions. This implies that Lm-CRLF3 is not involved in physiological maintenance of differentiated neurons, but specifically induces protective mechanisms upon Epo stimulation. However, LmCRLF3 is crucial for Epo-induced neuroprotection in vitro since a knock-down by RNAi abolished the neuroprotective effect of Epo in locust primary brain cell cultures. Our previous study focused on the holometabolous beetle $T$. castaneum and saw similar results concerning CRLF3 involvement. Given that locusts are hemimetabolous, these findings lead to the assumption that the last common ancestor of hemi- and holometabolous insects already employed CRLF3 as a neuroprotective receptor. However, its endogenous ligand is yet unknown. Since insects do not possess epo genes, the endogenous ligand has to be different from Epo but might share structural features with Epo and other class-I helical cytokines. The artificial activation of insect CRLF3 receptors by rhEpo is not surprising, because CRLF3 and EpoR both belong to group 1 of class I cytokine receptors and EpoR has already been shown to crossreact with thrombopoetin, the typical ligand of another receptor of that group (Rouleau et al., 2004).

In contrast to vertebrates, only few cytokines or cytokinelike peptides have been identified in insects (Duressa et al., 2015; Schrag et al., 2017; Matsumura et al., 2018). Expression of CRLF3 by locust hemocytes and brain cells may indicate multiple production sites of its endogenous ligand, since bloodbrain-barriers restrict the exchange of ions and soluble molecules (reviewed in DeSalvo et al., 2011). Potential production sites, that release the ligand into the circulation, are certain types of hemocytes, neurosecretory organs including the corpora allata as well as the corpora cardiaca, and the fat body. They contain and release also other cytokines involved in stress responses (Duressa et al., 2015; Matsumura et al., 2018).

As documented for many species from different orders, insects achieve extraordinary resistance to hypoxia by switching to anaerobic metabolic pathways and reduction of basal metabolic rates amongst other adaptations (reviewed by Hoback and Stanley, 2001). Hypoxia tolerance has also been reported for locusts (Arieli and Lehrer, 1988; Wegener and Moratzky, 1995; Greenlee and Harrison, 2004) and T. castaneum (Donahaye, 1990; Kharel et al., 2019), the two species in which CRLF3-mediated neuroprotection has been demonstrated. In comparison to mammalian neurons, where rather brief hypoxic episodes are sufficient to induce apoptosis, survival of locust and beetle neurons in vitro decreases only $20-30 \%$ even when challenged by prolonged ( $36 \mathrm{~h})$ and severe ( $<0.3 \%$ oxygen) hypoxia (this study; Miljus et al., 2014; Hahn et al., 2017). However, similar degrees of hypoxia tolerance have also been reported for specially adapted vertebrates (such as turtles and naked mole-rats; reviewed by Larson et al., 2014) and for some mammalian cell types in vitro (human SH-SY5Y neuroblastoma cells, Reich et al., 2008). Since DAPI nuclear staining, trypan blue accumulation and immunocytochemical detection of pro-apoptotic activated caspase-3 consistently identified dead or dying locust neurons (Gocht et al., 2009; Miljus et al., 2014; Heinrich et al., 2017), hypoxia-induced cell death is most likely not underestimated by our analysis of DAPI-labeled nuclear morphology. Whether and how CRLF3-induced adaptations may contribute to hypoxia tolerance in vivo will be a subject of our future studies.

The neuroprotective effect of rhEpo on locust neurons challenged by hypoxia, by the cellular toxin $\mathrm{H}-7$ or by serum deprivation has been characterized earlier. Its antiapoptotic mechanisms involve activation of JAK/STAT signaling, translation of anti-apoptotic factors and interference with caspase-activation but are independent of PI3K signaling (Miljus et al., 2014; Heinrich et al., 2017). At that time, the receptor mediating this effect was not known. It can be assumed, that these insights are transferable to neuroprotective CRLF3 signaling since the knock-down of CRLF3 in beetles and locusts brain cell cultures abolished Epo-mediated neuroprotection completely, suggesting that CRLF3 is the only neuroprotective Epo-receptor in these insect neurons. Furthermore, it is likely that even the neuroprotective but non-erythropoietic Epo variants (e.g., EV-3) and Epo like ligands activate CRLF3. Epo-induced endocytosis is reduced by pre-incubation to EV-3 indicating that EV-3 and Epo bind to the same receptor on locust neurons (Miljus et al., 2017).

Its sensitivity toward Epo treatment in insects and its high conservation throughout metazoans suggests CRLF3 as a potential mammalian neuroprotective Epo-receptor. The neuroprotective function of Epo has been well-investigated in vertebrates and Epo is even used in clinical trials as a treatment after ischemic stroke (Ehrenreich et al., 2009; Subiras et al., 2012; Habib et al., 2019; Simon et al., 2019). However, Epo treatment leads to various adverse side effects (e.g., thromboembolism, cardiovascular events) that mainly arise from its erythropoietic function in vertebrates (Jelkmann et al., 2008; Noguchi et al., 2008; Ehrenreich et al., 2009; Souvenir et al., 2015). Hence, developing drugs that specifically target CRLF3 might improve neuroprotective therapies.

\section{Outlook}

Current experiments focus on the identification of the endogenous ligand of CRLF3 and the characterization of mammalian CRLF3. Cytokines typically share low sequence and overall structural similarity which complicates analyses of their evolutionary origins (Beschin et al., 2001; Liongue and Ward, 2007). Instead of bioinformatic approaches based on sequence similarity, endogenous CRLF3 ligands may rather be identified by functional studies with fractionated tissue extracts from which potential ligands can be separated and molecularly identified (Watari et al., 2019). In addition, potential neuro- and 
cell protective functions of CRLF3 in Mammalia should be investigated also considering a putative involvement in the adaptive immune system.

\section{DATA AVAILABILITY STATEMENT}

Nucleotide sequences were submitted to GenBank with the submission numbers MN245516 and MN245517.

\section{AUTHOR CONTRIBUTIONS}

$\mathrm{NH}, \mathrm{LB}, \mathrm{NS}-\mathrm{D}, \mathrm{BM}, \mathrm{PN}$, and $\mathrm{RH}$ collected the experimental data. $\mathrm{NH}, \mathrm{LB}, \mathrm{BG}$, and $\mathrm{RH}$ conducted the data analysis and interpretation. $\mathrm{NH}$ and $\mathrm{SB}$ performed the phylogenetic analysis. $\mathrm{NH}, \mathrm{MG}$, and $\mathrm{RH}$ wrote the manuscript. $\mathrm{NH}$ and $\mathrm{RH}$ designed and supervised the study. All authors discussed the results and commented on the manuscript.

\section{FUNDING}

The project was funded by Deutsche Forschungsgemeinschaft (DFG) - Projektnummer 398214842. NH was partially supported

\section{REFERENCES}

Agrawal, A., Eastman, Q. M., and Schatz, D. G. (1998). Implications of transposition mediated by V(D)J-recombination proteins RAG1 and RAG2 for origins of antigen-specific immunity. Nature 394, 744-751. doi: 10.1038/29457

Altschul, S. F., Gish, W., Miller, W., Myers, E. W., and Lipman, D. J. (1990). Basic local alignment search tool. J. Mol. Biol. 215, 403-410. doi: 10.1006/jmbi.1990. 9999

Arieli, R., and Lehrer, C. (1988). Recording of locust breathing frequency by barometric method exemplified by hypoxic exposure. J. Insect Physiol. 34, 325-328. doi: 10.1016/0022-1910(88)90143-6

Atwal, J. K., Julie, P. G., Josh, S., Scott, S., Yan, W., Carla, S., et al. (2008). PirB is a functional receptor for myelin inhibitors of axonal regeneration. Science 322, 967-970. doi: 10.1126/science.1161151

Babonis, L. S., and Martindale, M. Q. (2016). Phylogenetic evidence for the modular evolution of metazoan signalling pathways. Philos. Trans. B 372:20150477. doi: $10.1098 /$ rstb.2015.0477

Bayne, C. J. (2003). Origins and evolutionary relationships between the innate and adaptive arms of immune systems. Integr. Comp. Biol. 43, 293-299. doi: 10.1093/icb/43.2.293

Ben Baruch-Morgenstern, N., Shik, D., Moshkovits, I., Itan, M., Karo-Atar, D., Bouffi, C., et al. (2014). Paired immunoglobulin-like receptor A is an intrinsic, self-limiting suppressor of IL-5-induced eosinophil development. Nat. Immunol. 15, 36-44. doi: 10.1038/ni.2757

Benjamini, Y., and Hochberg, Y. (1995). Controlling the False discovery rate: a practical and powerful approach to multiple testing. J. R. Stat. Soc. Ser. B 57, 289-300. doi: 10.1111/j.2517-6161.1995.tb02031.x

Berná, L., Alvarez-Valin, F., and D’Onofrio, G. (2009). How fast is the sessile Ciona? Comp. Funct. Genomics. 2006:875901. doi: 10.1155/2009/875901

Beschin, A., Bilej, M., Torreele, E., and De Baetselier, P. (2001). Cellular and molecular life sciences On the existence of cytokines in invertebrates. Cell. Mol. Life Sci. 58, 801-814. doi: 10.1007/pl00000901

Bonnas, C., Wüstefeld, L., Winkler, D., Kronstein-Wiedemann, R., Dere, E., Specht, K., et al. (2017). EV-3, an endogenous human erythropoietin isoform with distinct functional relevance. Sci. Rep. 7, 1-15. doi: 10.1038/s41598-01703167-0 by a grant from the Deutsche Forschungsgemeinschaft (DFG CRC 889, A1) to MG. SB was supported by a grant from the Deutsche Forschungsgemeinschaft (DFG 2930\3-1) to Sven Bradler.

\section{ACKNOWLEDGMENTS}

We thank Steffi Pauls for technical assistance, Sven Bradler for scientific advice and Debbra Y. Knorr for proofreading and language editing of the manuscript. Furthermore, we thank Gregor Bucher for introducing us to RNAi in Tribolium castaneum and Natascha Zhang for assistance with RACE PCR. We acknowledge the support by the Open Access Publication Funds of the Göttingen University. Finally, we owe our thanks to the reviewers for constructive comments which greatly improved the quality of the manuscript.

\section{SUPPLEMENTARY MATERIAL}

The Supplementary Material for this article can be found online at: https://www.frontiersin.org/articles/10.3389/fnmol. 2019.00251/full\#supplementary-material

Bosch, T. C. G., Klimovich, A., Domazet-Lošo, T., Gründer, S., Holstein, T. W., Jékely, G., et al. (2017). Back to the basics: cnidarians start to fire. Trends Neurosci. 40, 92-105. doi: 10.1016/j.tins.2016.11.005

Boulay, J. L., O'Shea, J. J., and Paul, W. E. (2003). Molecular phylogeny within type I cytokines and their cognate receptors. Immunity 19, 159-163. doi: 10.1016/s1074-7613(03)00211-5

Brines, M., and Cerami, A. (2005). Emerging biological roles for erythropoietin in the nervous system. Nat. Rev. Neurosci. 6, 484-494. doi: 10.1038/nrn1687

Brines, M., and Cerami, A. (2006). Discovering erythropoietin's extrahematopoietic functions: biology and clinical promise. Kidney Int. 70, 246-250. doi: 10.1038/sj.ki.5001546

Brines, M., Grasso, G., Fiordaliso, F., Sfacteria, A., Ghezzi, P., Fratelli, M., et al. (2004). Erythropoietin mediates tissue protection through an erythropoietin and common -subunit heteroreceptor. Proc. Natl. Acad. Sci. U.S.A. 101, 1490714912. doi: 10.1073/pnas.0406491101

Brines, M., Patel, N. S. A., Villa, P., Brines, C., Mennini, T., De Paola, M., et al. (2008). Nonerythropoietic, tissue-protective peptides derived from the tertiary structure of erythropoietin. Proc. Natl. Acad. Sci. U.S.A. 105, 10925-10930. doi: 10.1073/pnas.0805594105

Brown, M. R., Crim, J. W., Arata, R. C., Cai, H. N., Chun, C., and Shen, P. (1999). Identification of a Drosophila brain-gut peptide related to the neuropeptide Y family. Peptides 20, 1035-1042. doi: 10.1016/s0196-9781(99)00 097-2

Brunet, F. G., Volff, J. N., and Schartl, M. (2016). Whole genome duplications shaped the receptor tyrosine kinase repertoire of jawed vertebrates. Genome Biol. Evol. 8, 1600-1613. doi: 10.1093/gbe/evw103

Cirulli, E. T., Lasseigne, B. N., Petrovski, S., Sapp, P. C., Dion, P. A., Leblond, C. S., et al. (2015). Exome sequencing in amyotrophic lateral sclerosis identifies risk genes and pathways. Science 347, 1436-1441. doi: 10.1126/science.aaa 3650

Dang, C., Gottschling, M., Manning, K., O’Currain, E., Schneider, S., Sterry, W., et al. (2006). Identification of dysregulated genes in cutaneous squamous cell carcinoma. Oncol. Rep. 16, 513-519.

DeSalvo, M. K., Mayer, N., Mayer, F., and Bainton, R. J. (2011). Physiologic and anatomic characterization of the brain surface glia barrier of Drosophila. Glia 59, 1322-1340. doi: 10.1002/glia.21147 
Donahaye, E. (1990). Laboratory selection of resistance by the red flour beetle, Tribolium castaneum (Herbst), to an atmosphere of low oxygen concentration. Phytoparasitica 18, 189-202. doi: 10.1007/BF02980989

Donnadieu, E., Revy, P., and Trautmann, A. (2001). Imaging T-cell antigen recognition and comparing immunological and neuronal synapses. Immunology 103, 417-425. doi: 10.1046/j.1365-2567.2001.01268.x

Dunn, C. W., Giribet, G., Edgecombe, G. D., and Hejnol, A. (2014). Animal phylogeny and its evolutionary implications. Annu. Rev. Ecol. Evol. Syst. 45, 371-395. doi: 10.1146/annurev-ecolsys-120213-091627

Duressa, T. F., Boonen, K., Hayakawa, Y., and Huybrechts, R. (2015). Identification and functional characterization of a novel locust peptide belonging to the family of insect growth blocking peptides. Peptides 74, 23-32. doi: 10.1016/j.peptides. 2015.09.011

Dzik, J. M. (2010). The ancestry and cumulative evolution of immune reactions. Acta Biochim. Pol. 57, 443-466.

Ehrenreich, H., Weissenborn, K., Prange, H., Schneider, D., Weimar, C., Wartenberg, K., et al. (2009). Recombinant human erythropoietin in the treatment of acute ischemic stroke. Stroke 40, e647-e656. doi: 10.1161/ STROKEAHA.109.564872

Erbayraktar, S., Grasso, G., Sfacteria, A., Xie, Q., Coleman, T., Kreilgaard, M., et al. (2003). Asialoerythropoietin is a nonerythropoietic cytokine with broad neuroprotective activity in vivo. Proc. Natl. Acad. Sci. U.S.A. 100, 6741-6746. doi: $10.1073 /$ pnas. 1031753100

Gautam, M., Noakes, P. G., Moscoso, L., Rupp, F., Scheller, R. H., Merlie, J. P., et al. (1996). Defective neuromuscular synaptogenesis in agrin-deficient mutant mice. Cell 85, 525-535. doi: 10.1016/s0092-8674(00)81253-2

Ghezzi, P., and Conklin, D. (2013). Tissue-protective cytokines: structure and evolution. Methods Mol. Biol. 982, 43-58. doi: 10.1007/978-1-62703-308-4_3

Gocht, D., Wagner, S., and Heinrich, R. (2009). Recognition, presence, and survival of locust central nervous glia in situ and in vitro. Microsc. Res. Tech. 72, 385-397. doi: $10.1002 /$ jemt.20683

Greenlee, K. J., and Harrison, J. F. (2004). Development of respiratory function in the American locust Schistocerca americana. J. Exp. Biol. 207, 509-517. doi: $10.1242 /$ jeb.00766

Grimmelikhuijzen, C. J. P., Leviev, I., and Carstensen, K. (1996). Peptides in the nervous systems of cnidarians: structure, function, and biosynthesis. Int. Rev. Cytol. 167, 37-89. doi: 10.1016/s0074-7696(08)61345-5

Habib, P., Stamm, A. S., Zeyen, T., Noristani, R., Slowik, A., Beyer, C., et al. (2019). EPO regulates neuroprotective Transmembrane BAX Inhibitor-1 Motif-containing (TMBIM) family members GRINA and FAIM2 after cerebral ischemia-reperfusion injury. Exp. Neurol. 320:112978. doi: 10.1016/j.expneurol. 2019.112978

Habibi, L., Ebtekar, M., and Jameie, S. B. (2009). Immune and nervous systems share molecular and functional similarities: memory storage mechanism. Scand. J. Immunol. 69, 291-301. doi: 10.1111/j.1365-3083.2008.02215.x

Hahn, N., Knorr, D., Liebig, J., Wüstefeld, L., Peters, K., Büscher, M., et al. (2017). The human orphan cytokine receptor CRLF3 is a neuroprotective erythropoietin receptor in insects. Front. Mol. Neurosci. 10:223. doi: 10.3389/ fnmol.2017.00223

Hashimoto, Y., Muramatsu, K., Kunii, M., Yoshimura, S. I., Yamada, M., Sato, T., et al. (2012). Uncovering genes required for neuronal morphology by morphology-based gene trap screening with a revertible retrovirus vector. FASEB J. 26, 4662-4674. doi: 10.1096/fj.12-207530

Heinrich, R., Guenther, V., and Miljus, N. (2017). Erythropoietin-mediated neuroprotection in insects suggests a prevertebrate evolution of erythropoietinlike signaling. Vitam. Horm. 105, 181-196. doi: 10.1016/bs.vh.2017.0 2.004

Hoang, D. T., Chernomor, O., von Haeseler, A., Minh, B. Q., and Vinh, L. S. (2018). UFBoot2: improving the ultrafast bootstrap approximation. Mol. Biol. Evol. 35, 518-522. doi: 10.1093/molbev/msx281

Hoback, W. W., and Stanley, D. W. (2001). Insects in hypoxia. J. Insect Physiol. 47, 533-542. doi: 10.1016/s0022-1910(00)00153-0

Holdsworth, S. R., and Gan, P. Y. (2015). Cytokines: names and numbers you should care about. Clin. J. Am. Soc. Nephrol. 10, 2243-2254. doi: 10.2215/CJN. 07590714

Hothorn, T., Hornik, K., van de Wiel, M. A., and Zeileis, A. (2006). A lego system for conditional inference. Am. Stat. 60, 257-263. doi: 10.1198/ $000313006 \times 118430$
Hothorn, T., Hornik, K., van de Wiel, M. A., and Zeileis, A. (2008). Implementing a class of permutation tests: the coin package. J. Stat. Softw. 28, 1-23. doi: 10.18637/jss.v028.i08

Irie, N., Satoh, N., and Kuratani, S. (2018). The phylum Vertebrata: a case for zoological recognition. Zool. Lett. 4:32. doi: 10.1186/s40851-018-0114-y

Jékely, G., Paps, J., and Nielsen, C. (2015). The phylogenetic position of ctenophores and the origin(s) of nervous systems. Evodevo 6:1. doi: 10.1186/ 2041-9139-6-1

Jelkmann, W. (2011). Regulation of erythropoietin production. J. Physiol. 589, 1251-1258. doi: 10.1113/jphysiol.2010.195057

Jelkmann, W., Bohlius, J., Hallek, M., and Sytkowski, A. J. (2008). The erythropoietin receptor in normal and cancer tissues. Crit. Rev. Oncol. Hematol. 67, 39-61. doi: 10.1016/j.critrevonc.2008.03.006

Kapitonov, V. V., and Koonin, E. V. (2015). Evolution of the RAG1-RAG2 locus: both proteins came from the same transposon. Biol. Direct 10:20. doi: 10.1186/ s13062-015-0055-8

Kasahara, M. (2007). The 2R hypothesis: an update. Curr. Opin. Immunol. 19, 547-552. doi: 10.1016/j.coi.2007.07.009

Kerschensteiner, M., Meinl, E., and Hohlfeld, R. (2009). Neuro-immune crosstalk in CNS diseases. Neuroscience 158, 1122-1132. doi: 10.1016/j.neuroscience. 2008.09.009

Khan, A. A., Bose, C., Yam, L. S., Soloski, M. J., and Rupp, F. (2001). Physiological regulation of the immunological synapse by agrin. Science 292, 1681-1686. doi: 10.1126/science.1056594

Kharel, K., Mason, L. J., Murdock, L. L., and Baributsa, D. (2019). Efficacy of hypoxia against Tribolium castaneum (Coleoptera: Tenebrionidae) throughout ontogeny. J. Econ. Entomol. 112, 1463-1468. doi: 10.1093/jee/toz019

Kioussis, D., and Pachnis, V. (2009). Immune and nervous systems: more than just a superficial similarity? Immunity 31, 705-710. doi: 10.1016/j.immuni.2009.0 9.009

Larson, J., Drew, K. L., Folkow, L. P., Milton, S. L., and Park, T. J. (2014). No oxygen? No problem! Intrinsic brain tolerance to hypoxia in vertebrates. J. Exp. Biol. 217, 1024-1039. doi: 10.1242/jeb.085381

Laumer, C. E., Fernández, R., Lemer, S., Combosch, D., Kocot, K. M., Riesgo, A., et al. (2019). Revisiting metazoan phylogeny with genomic sampling of all phyla. Proc. R. Soc. B Biol. Sci. 286:20190831. doi: 10.1098/rspb.2019. 0831

Leist, M., Ghezzi, P., Grasso, G., Bianchi, R., Villa, P., Fratelli, M., et al. (2004). Derivatives of erythropoietin not erythropoietic. Science 305, 239-243.

Levite, M. (2008). Neurotransmitters activate T-cells and elicit crucial functions via neurotransmitter receptors. Curr. Opin. Pharmacol. 8, 460-471. doi: 10.1016/j. coph.2008.05.001

Liongue, C., Sertori, R., and Ward, A. C. (2016). Evolution of cytokine receptor signaling. J. Immunol. 197, 11-18. doi: 10.4049/jimmunol.1600372

Liongue, C., and Ward, A. C. (2007). Evolution of class I cytokine receptors. BMC Evol. Biol. 7:120. doi: 10.1186/1471-2148-7-120

Livak, K. J., and Schmittgen, T. D. (2001). Analysis of relative gene expression data using real-time quantitative PCR and the $2-\Delta \Delta C T$ method. Methods 25, 402-408. doi: 10.1006/meth.2001.1262

Luo, Y., Wang, X., Wang, X., Yu, D., Chen, B., and Kang, L. (2013). Differential responses of migratory locusts to systemic RNA interference via doublestranded RNA injection and feeding. Insect Mol. Biol. 22, 574-583. doi: 10.1111/ imb. 12046

Ma, Z., Guo, W., Guo, X., Wang, X., and Kang, L. (2011). Modulation of behavioral phase changes of the migratory locust by the catecholamine metabolic pathway. Proc. Natl. Acad. Sci. U.S.A. 108, 3882-3887. doi: 10.1073/pnas.1015098108

Mamta, B., and Rajam, M. V. (2017). RNAi technology: a new platform for crop pest control. Physiol. Mol. Biol. Plants 23, 487-501. doi: 10.1007/s12298-0170443-x

Mangiafico, S. (2019). rcompanion: Functions to Support Extension Education Program Evaluation. Package Version 2.1.7.

Matsumura, T., Nakano, F., Matsumoto, H., Uryu, O., and Hayakawa, Y. (2018). Identification of a cytokine combination that protects insects from stress. Insect Biochem. Mol. Biol. 97, 19-30. doi: 10.1016/j.ibmb.2018.04.002

Meola, D., Huang, Z., and Petitto, J. M. (2013). Selective neuronal and brain regional expession of IL-2 in IL2P 8-GFP transgenic mice: relation to sensorimotor gating. J. Alzheimers Dis. Park. 3:1000127. doi: 10.4172/21610460.1000127 
Miljus, N., Heibeck, S., Jarrar, M., Micke, M., Ostrowski, D., Ehrenreich, H., et al. (2014). Erythropoietin-mediated protection of insect brain neurons involves JAK and STAT but not PI3K transduction pathways. Neuroscience 258, 218-227. doi: 10.1016/j.neuroscience.2013.11.020

Miljus, N., Massih, B., Weis, M. A., Rison, J. V., Bonnas, C. B., Sillaber, I., et al. (2017). Neuroprotection and endocytosis: erythropoietin receptors in insect nervous systems. J. Neurochem. 141, 63-74. doi: 10.1111/jnc. 13967

Minh, B. Q., Nguyen, M. A. T., and Von Haeseler, A. (2013). Ultrafast approximation for phylogenetic bootstrap. Mol. Biol. Evol. 30, 1188-1195. doi: 10.1093/molbev/mst024

Misof, B., Liu, S., Meusemann, K., Peters, R. S., Donath, A., Mayer, C., et al. (2014). Phylogenomics resolves the timing and pattern of insect evolution. Science 346, 763-767. doi: 10.1126/science. 1257570

Moroz, L. L., and Kohn, A. B. (2016). Independent origins of neurons and synapses: insights from ctenophores. Philos. Trans. R. Soc. B Biol. Sci. 371:20150041. doi: 10.1098/rstb.2015.0041

Muraguchi, B. Y. A., Kehrl, J. H., Longo, D. A. N. L., Volkman, D. J., Smith, K. A., and Fauci, A. S. (1985). Interleukin 2 receptors on human B cells. Implications for the role of interleukin 2 in human B cell function. J. Exp. Med. 161, 181-197. doi: $10.1084 /$ jem.161.1.181

Nguyen, L. T., Schmidt, H. A., Von Haeseler, A., and Minh, B. Q. (2015). IQ-TREE: a fast and effective stochastic algorithm for estimating maximumlikelihood phylogenies. Mol. Biol. Evol. 32, 268-274. doi: 10.1093/molbev/ms u300

Niu, J., Taning, C. N. T., Christiaens, O., Smagghe, G., and Wang, J. J. (2018). "Rethink RNAi in Insect Pest Control: Challenges and Perspectives," in Advances in Insect Physiology, Vol. 55, ed. G. Smagghe, (Cambridge, MA: Elsevier Ltd.), 1-7. doi: 10.1016/bs.aiip.2018.07.003

Noguchi, C. T., Wang, L., Rogers, H. M., Teng, R., and Jia, Y. (2008). Survival and proliferative roles of erythropoietin beyond the erythroid lineage. Expert Rev. Mol. Med. 10, 1-27. doi: 10.1017/S1462399408000860

Oettinger, M. A., Schatz, D. G., Gorka, C., and Baltimore, D. (1990). RAG-I1 and RAG-2, adjacent genes that synergistically activate $\mathrm{V}(\mathrm{D}) \mathrm{J}$ recombination. Science 248, 1517-1523. doi: 10.1126/science.2360047

Ogunshola, O. O., and Bogdanova, A. Y. (2013). Tissue-protective cytokines. Methods Mol. Biol. 982, 13-41.

Ostrowski, D., Ehrenreich, H., and Heinrich, R. (2011). Erythropoietin promotes survival and regeneration of insect neurons in vivo and in vitro. Neuroscience 188, 95-108. doi: 10.1016/j.neuroscience.2011.05.018

Pradeep, S., Huang, J., Mora, E. M., Nick, A. M., Cho, M. S., Wu, S. Y., et al. (2016). Erythropoietin stimulates tumor growth via EphB4. Cancer Cell 28, 610-622. doi: 10.1016/j.ccell.2015.09.008

R Core Team, (2019). R: A Language and Environment for Statistical Computing. Vienna: R Foundation for Statistical Computing.

Ransohoff, R. M. (2009). Chemokines and chemokine receptors: standing at the crossroads of immunobiology and neurobiology. Immunity 31, 711-721. doi: 10.1016/j.immuni.2009.09.010

Rast, J. P., Anderson, M. K., Strong, S. J., Luer, C., Litman, R. T., and Litman, G. W. (1997). $\alpha, \beta, \gamma, \delta \mathrm{T}$ cell antigen receptor genes arose early in vertebrate phylogeny. Immunity 6, 1-11. doi: 10.1016/S1074-7613(00)80237-X

Reich, D. M., Hau, S., Stahl, T., Scholz, M., Naumann, W., Emmrich, F., et al. (2008). Neuronal hypoxia in vitro: investigation of therapeutic principles of HUCB-MNC and CD133+ stem cells. BMC Neurosci. 9:91. doi: 10.1186/14712202-9-91

Ren, D., Cai, Z., Song, J., Wu, Z., and Zhou, S. (2014). DsRNA uptake and persistence account for tissue-dependent susceptibility to RNA interference in the migratory locust, Locusta migratoria. Insect Mol. Biol. 23, 175-184. doi: 10.1111/imb. 12074

Rouleau, C., Cui, K., and Feldman, L. (2004). A functional erythropoietin receptor is necessary for the action of thrombopoietin on erythroid cells lacking c-mpl. Exp. Hematol. 32, 140-148. doi: 10.1016/j.exphem.2003.10.015

RStudio Team, (2018). RStudio: Integrated Development for R. Boston, MA: RStudio, Inc.

Sarrion-Perdigones, A., Vazquez-Vilar, M., Palaci, J., Castelijns, B., Forment, J., Ziarsolo, P., et al. (2013). GoldenBraid 2.0: a comprehensive DNA assembly framework for plant synthetic biology. Plant Physiol. 162, 1618-1631. doi: $10.1104 /$ pp.113.217661
Schatz, D. G. (2004). Antigen receptor genes and the evolution of a recombinase. Semin. Immunol. 16, 245-256. doi: 10.1016/j.smim.2004.08.004

Schindelin, J., Arganda-Carreras, I., Frise, E., Kaynig, V., Longair, M., Pietzsch, T., et al. (2012). Fiji: an open-source platform for biological-image analysis. Nat. Methods 9, 676-682. doi: 10.1038/nmeth.2019

Schrag, L. G., Cao, X., Herrera, A. I., Wang, Y., Jiang, H., and Prakash, O. (2017). Solution structure and expression profile of an insect cytokine: Manduca sexta stress response peptide-2. Protein Pept. Lett. 24, 3-11. doi: 10.2174/ 0929866524666161121142840

Simon, F., Floros, N., Ibing, W., Schelzig, H., and Knapsis, A. (2019). Neurotherapeutic potential of erythropoietin after ischemic injury of the central nervous system. Neural Regen. Res. 14, 1309-1312. doi: 10.4103/1673-5374.25 3507

Singh, I. K., Singh, S., Mogilicherla, K., Shukla, J. N., and Palli, S. R. (2017). Comparative analysis of double-stranded RNA degradation and processing in insects. Sci. Rep. 7:17059. doi: 10.1038/s41598-017-17134-2

Song, H., Fan, Y., Zhang, J., Cooper, A. M. W., Silver, K., Li, D., et al. (2019). Contributions of dsRNases to differential RNAi efficiencies between the injection and oral delivery of dsRNA in Locusta migratoria. Pest Manag. Sci. 75, 1707-1717. doi: 10.1002/ps.5291

Souvenir, R., Doycheva, D., Zhang, J., and Tang, J. (2015). Erythropoietin in stroke therapy: friend or foe. Curr. Med. Chem. 22, 1205-1213. doi: 10.2174/ 0929867322666150114152134

Subiras, N., Del Barco, D. G., and Coro-Antich, R. M. (2012). Erythropoietin: still on the neuroprotection road. Ther. Adv. Neurol. Disord. 5, 161-173. doi: $10.1177 / 1756285611434926$

Takezaki, N., and Nishihara, H. (2016). Resolving the phylogenetic position of coelacanth: the closest relative is not always the most appropriate outgroup. Genome Biol. Evol. 8, 1208-1221. doi: 10.1093/gbe/evw071

Um, M., Gross, A. W., and Lodish, H. F. (2007). A "classical" homodimeric erythropoietin receptor is essential for the antiapoptotic effects of erythropoietin on differentiated neuroblastoma SH-SY5Y and pheochromocytoma PC-12 cells. Cell. Signal. 19, 634-645. doi: 10.1016/j.cellsig.2006.08.014

Unger, E. F., Thompson, A. M., Blank, M. J., and Temple, R. (2010). Erythropoiesisstimulating agents - time for a reevaluation. N. Engl. J. Med. 362, 189-192. doi: 10.1056/nejmp0912328

Van Hiel, M. B., Van Wielendaele, P., Temmerman, L., Van Soest, S., Vuerinckx, K., Huybrechts, R., et al. (2009). Identification and validation of housekeeping genes in brains of the desert locust Schistocerca gregaria under different developmental conditions. BMC Mol. Biol. 10:56. doi: 10.1186/1471-2199-1056

Vogel, E., Santos, D., Mingels, L., Verdonckt, T. W., and Broeck, J. V. (2019). RNA interference in insects: protecting beneficials and controlling pests. Front. Physiol. 10:1912. doi: 10.3389/fphys.2018.01912

Wang, K., Peng, Y., Pu, J., Fu, W., Wang, J., and Han, Z. (2016). Variation in RNAi efficacy among insect species is attributable to dsRNA degradation in vivo. Insect Biochem. Mol. Biol. 77, 1-9. doi: 10.1016/j.ibmb.2016.07.007

Watari, H., Nakajima, H., Atsuumi, W., Nakamura, T., Nanya, T., Ise, Y., et al. (2019). A novel sponge-derived protein thrombocorticin is a new agonist for thrombopoietin receptor. Comp. Biochem. Physiol. Part-C Toxicol. Pharmacol. 221, 82-88. doi: 10.1016/j.cbpc.2019.04.003

Wegener, G., and Moratzky, T. (1995). Hypoxia and anoxia in insects: microcalorimetric studies on two species (Locusta migratoria and Manduca sexta) showing different degrees of anoxia tolerance. Thermochim. Acta 251, 209-218. doi: 10.1016/0040-6031(94)02009-d

Westfall, I. A. (1996). Ultrastructure of synapses in the first-evolved nervous systems. J. Neurocytol. 25, 735-746. doi: 10.1007/bf02284838

Wheway, J., Herzog, H., and Mackay, F. (2007). NPY and receptors in immune and inflammatory diseases. Curr. Top. Med. Chem. 7, 1743-1752. doi: 10.2174/ 156802607782341046

Wong, T. K. F., Jermiin, L. S., Minh, B. Q., Kalyaanamoorthy, S., and von Haeseler, A. (2017). ModelFinder: fast model selection for accurate phylogenetic estimates. Nat. Methods 14, 587-589. doi: 10.1038/nmeth. 4285

Wyder, S., Kriventseva, E. V., Schröder, R., Kadowaki, T., and Zdobnov, E. M. (2007). Quantification of ortholog losses in insects and vertebrates. Genome Biol. 8:R242. doi: 10.1186/gb-2007-8-11-r242 
Xie, J., Li, S., Zhang, W., and Xia, Y. (2019). RNAi-knockdown of the Locusta migratoria nuclear export factor protein results in insect mortality and alterations in gut microbiome. Pest Manag. Sci. 75, 1383-1390. doi: 10.1002/ ps.5258

Yang, F., Xu, Y.-P., Li, J., Duan, S.-S., Fu, Y.-J., Zhang, Y., et al. (2009). Cloning and characterization of a novel intracellular protein p48.2 that negatively regulates cell cycle progression. Int. J. Biochem. Cell Biol. 41, 2240-2250. doi: 10.1016/j.biocel.2009.04.022

Yoshida, R., Fukumizu, K., and Vogiatzis, C. (2019). Multilocus phylogenetic analysis with gene tree clustering. Ann. Oper. Res. 276, 293-313. doi: 10.1007/ s10479-017-2456-9

Zhang, J., Wang, Y., Chu, Y., Su, L., Gong, Y., Zhang, R., et al. (2006). Agrin is involved in lymphocytes activation that is mediated by $\alpha$-dystroglycan. FASEB J. 20, 50-58. doi: 10.1096/fj.04-3303com
Conflict of Interest: RH is a consultant for Epomedics GmbH, Göttingen, Germany.

The remaining authors declare that the research was conducted in the absence of any commercial or financial relationships that could be construed as a potential conflict of interest.

Copyright (c) 2019 Hahn, Büschgens, Schwedhelm-Domeyer, Bank, Geurten, Neugebauer, Massih, Göpfert and Heinrich. This is an open-access article distributed under the terms of the Creative Commons Attribution License (CC BY). The use, distribution or reproduction in other forums is permitted, provided the original author(s) and the copyright owner(s) are credited and that the original publication in this journal is cited, in accordance with accepted academic practice. No use, distribution or reproduction is permitted which does not comply with these terms. 


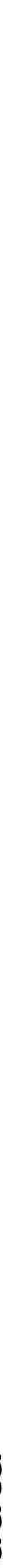




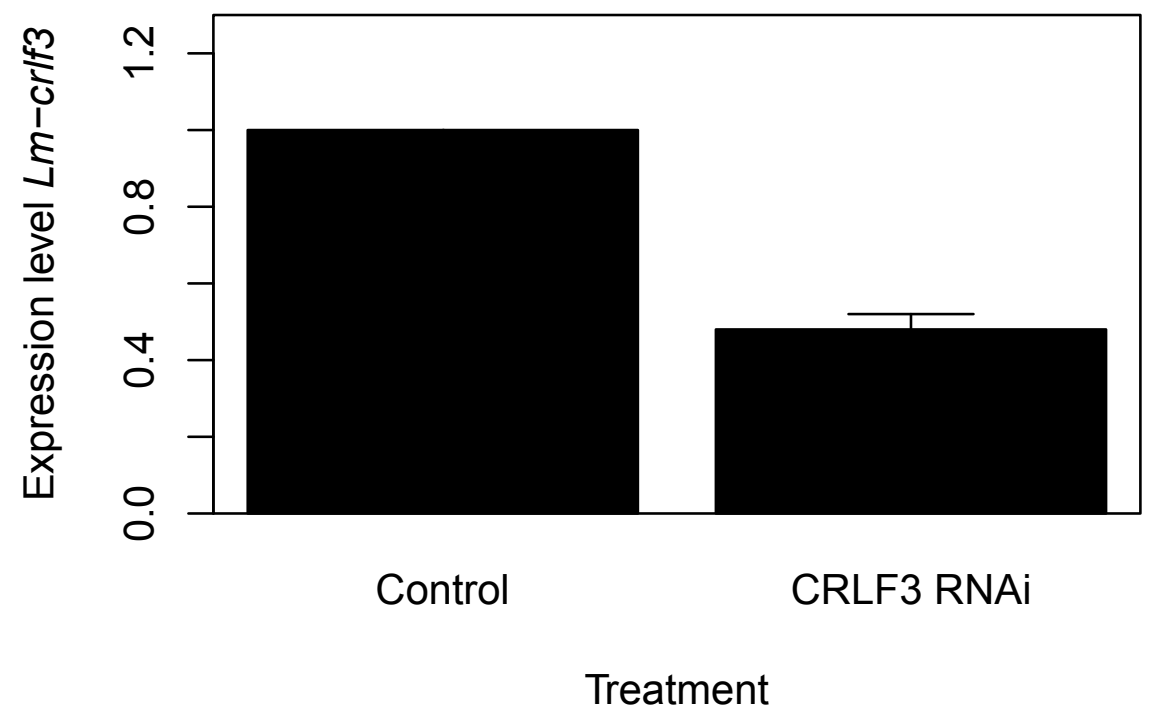

Supplementary Figure S2: Verification of Lm-crlf3 knock-down by qRTPCR after 5 days of soaking RNAi. Cells were incubated with injection buffer (control) or dsRNA targeting Lm-crlf3 fragment 1 . Mean $=0.48$, standard deviation $=0.04, \mathrm{~N}=2$. 


\begin{tabular}{|l|c|c|}
\hline \multicolumn{1}{|c|}{ Normoxia } & & \\
\hline days & $12 \mathrm{~h}$ & $36 \mathrm{~h}$ \\
\hline dsRNA $10 \mathrm{ng} / \mu \mathrm{l}$ & & \\
& $32 \mathrm{ng} / \mathrm{ml} \mathrm{rhEpo}$
\end{tabular} DAPI

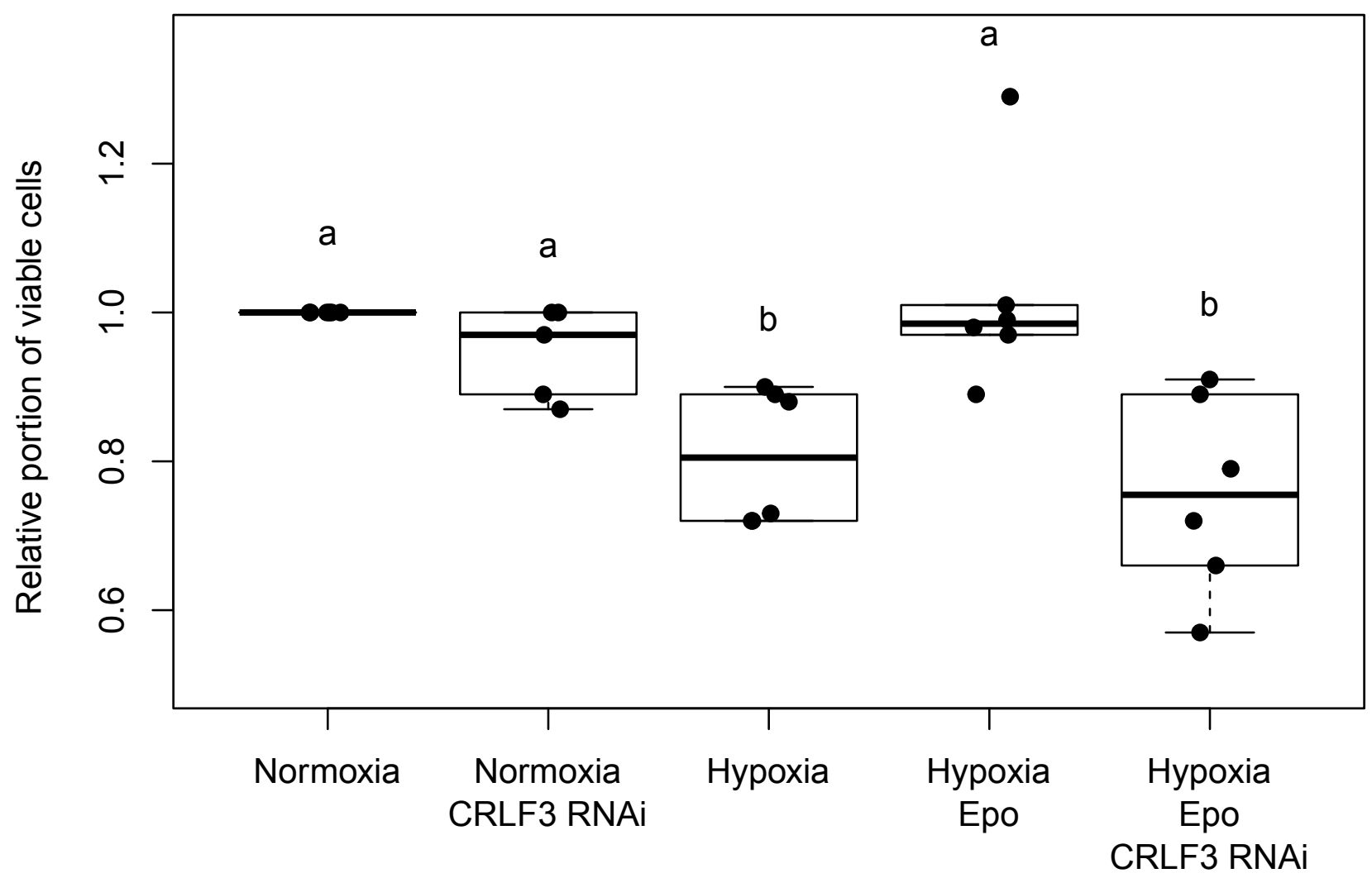

Supplementary Figure S3: Survival of L. migratoria primary brain neurons in normoxia, hypoxia and after knock-down of $L m$-CRLF3 expression with fragment 2. Cell survival was assessed by DAPI staining on day 7 to evaluate the impact of hypoxia $(36 \mathrm{~h})$, rhEpo $(32 \mathrm{ng} / \mathrm{ml})$ and RNAi-mediated knock-down of Lm-CRLF3 production (10 ng/ $\mu \mathrm{l}$ dsRNA targeting fragment 2$)$. Hypoxia significantly decreases cell viability but treatment with rhEpo prevents neurons from hypoxia-induced apoptosis. The neuroprotective effect of rhEpo is absent following knock-down of Lm-CRLF3 expression. Knocking down Lm-CRLF3 per se has no impact on cell viability in normoxia. $\mathrm{N}=6$ except normoxia $\mathrm{RNAi} N=5 ; 28,216$ cells counted. Statistics: Permutation test with Benjamini-Hochberg correction. Groups that do not share a letter $(a, b)$ are significantly different with at least $\mathrm{p}<0.05$. Boxplots are complemented by black dots that represent individual experiments. 


\section{Supplementary File S1}

\section{Full-length mRNA Lm-crlf3 (Accession Number MN245516)}

ACATGGGGGTAATCCTGATGTTTTCTGAGGGACATTTGAACTAGTAGGAAAATAAGCGGATTGTTTAAG TTTGCTCTAAGTATTCTTCTAAAGGAGGATATGAAAAGATTTATGAGCTCTGAAAAGTCTACTATGTGGTT GAAACGATATTTTTCCCGCTCCTGTAGTGTGCCGGAAATTAAGATTTTCTGAAGGCGTGGCAGATTTTCAT TAAATTTTTAAATATTCGTTTAATTTGCTTTCTTTGATTGAAATGGAACAGTCAAAGTCAAATAGTGTTTTT GTCGAAGCTGTCGATGCTGCTGAGGAATATGTGAAAAAATTGGAAGATCTACTGAAAACCCTTACTGAA GCAGAACATCAAGTGAAGCACAGTGCCAAGCAGGCAAGAGCAGATGTCAATTTTGCATTGGACACACTA GCAGCAACTTTCACTAATGCTTTAAAGCGAAGGCGAAGCATGCTCCTTGAAGAAGTGGATCGTTTGTGTA GTGAAGGACTGGAACCTCTAATTGAATGTAGAGATCTTATTACTACTCAAATGCAAGTGGCCCACAGCTA TGCTAATGAAGGGCGGCATTCTCTAAATGGCAGTAACAAATGTCCAGTTGCTGAAAGCTGGGATGACTA CACTCACTCATATGCAGAGTGTGGAGCTGCATTTCTTGGAAGGTTGCCAGCAGTCCCAAATTTGGAAGAT ATTCCTGGTGTGCAGTTTGAATGCTGTCTACATGCCATTGAAGCTGACTTGGTTCATGCTGTTGAGAGGG TTGGCAGTGTGTCACGACTTGGACCTGTCCAAATTTGTGCTGTTGAAGAGAAACCTGGAGCTCTGCTGGT CCATTGGCAGCAGGTAGAGACAGAGAAACCAGCAGAAATTGGCTCATTCAGGCTACAACGAGCATACG GAGATGCACGTGGCCGTCGTGAGTTAGAAGCCAATTTCCACGATGAGTATGTGGGACCTGAATGTCACC ACCTTATACGCAATCTGCGACCAAAGGAACCAGTCACTCTGCGAGTCTGTTGCCGTGAAGACAGTGAGCT TGCACCGTGGAGTACTTGGTCTGTGTTACATGTTGCTGCAACCTCACTACCTCCATTCTGCTGGGAGGGA ACAAATCAGAACTACACTATAACAAATGAGAAACAACTGGCCACAAAAAGCACCGCAGAAACGTCTGTC CTTTTTTCATGTGGGCCACAATTTGGACCAGGACATGCAGTGGAATTCACTGTTCTAGAATGTGGTGCAG GATGTAGTGACGAAGGTCTGGCTCTTGCCGATCACCATTTTAGTGATGAAAATCTTCTCCAGCCTGGGGT AATATTCGTTAATGCACAAGGAAGTGTGTTTGTGGATGGCAAGGGAAAGACTACCAAATTACCTCCCTTA GAAAAAGGCTCAAAACTATGTTTCACTTGTGAACATGTCCGCAGTAGTAAGGTCCGTATCCACATTGACA GTGGTAATAAGACTGTAGCGTATGACTGGAATGTTAGTAGTCCACTGCAAAAACTATTTTTTGCTATCTCT TTTGGACAAGTTGGCTGGAAGGTGTTGGTTGAATAAGAAAAATGGTAATTACAGATTACTTCACAAAAA AATTAAGGAATTTATATTTTGCTACAATTATACTTTTATACTGTAGCAGCCTTGAAGGTACCAMCAAGCA GTCTCATAATTTTTTGTTGTTCATCCCATCGATACTATACTACTTCCAATGTGTTCAGTTTTCAGTACGTGGA TATTTCATTCGTTGGCTGTAATTTTTAAATAGTGTCAGTTATCCAATTAGGTAACCTTCCAAAAAGCAGTTA TCCATTGGGATAATTTATTTTGTGTGTGTGTGTGTGTGTGTGTGTGTGTGTGTGTGTTTGTTTTTTAATTTT GTGTTTGAGTGTTATTATTTGAATYTGAATACCAATTTGTTTTTGTATCACTCTATTTTCATTTGGTGGTTTT TTTCCTGATGCTTAAACGTTTAGCAGAATTAGCATAATAGTTTTTTYTTGATGGTCAATGCTTTATTGYTAT GATAGAATWTAATATACCCCTAATTGCTTACATACCTGKGAAWATACATTATATWTCTTACCCTTTTGTT TGTTMCCCCTTTTTAWAATTATGTTAGKGKGATTAAGGTAAAACTTCMCATTTCYTAATCMCTAAATWTT TCCAAGTSCCTTGCTTTAASCMCTYCMCTGTGTTGTTGTTAATTTTTTTTMCCTAAATGKGKGAAGKGTTT GTMCAACCAATTCATGTAGKGTYTAAAGGACAAYTAGKGCATAATTTWTTGAMCATTYTATACTGCTTTY TTGACAMCATTGGKGTTCWTYTCYTYTGAACAAATTGTACATAGGKGAMCMCTTAAATTWTTTAMCAG ATGTTTTTTYTGRGAAATGTTATATATAMCAATTMCAATATATATATAAAATTWTTAAAAAATTGTTATG CATTTTGMCAAAAWAAAGAATTTTTAAAATAAAAAAAAAAAAAAAAAAAAAAAAAAAAAAAAAAGT

\section{CDS Lm-crlf3}

TTGCTTTCTTTGATTGAAATGGAACAGTCAAAGTCAAATAGTGTTTTTGTCGAAGCTGTCGATGCTGCTGA GGAATATGTGAAAAAATTGGAAGATCTACTGAAAACCCTTACTGAAGCAGAACATCAAGTGAAGCACAG TGCCAAGCAGGCAAGAGCAGATGTCAATTTTGCATTGGACACACTAGCAGCAACTTTCACTAATGCTTTA 
AAGCGAAGGCGAAGCATGCTCCTTGAAGAAGTGGATCGTTTGTGTAGTGAAGGACTGGAACCTCTAATT GAATGTAGAGATCTTATTACTACTCAAATGCAAGTGGCCCACAGCTATGCTAATGAAGGGCGGCATTCTC TAAATGGCAGTAACAAATGTCCAGTTGCTGAAAGCTGGGATGACTACACTCACTCATATGCAGAGTGTG GAGCTGCATTTCTTGGAAGGTTGCCAGCAGTCCCAAATTTGGAAGATATTCCTGGTGTGCAGTTTGAATG CTGTCTACATGCCATTGAAGCTGACTTGGTTCATGCTGTTGAGAGGGTTGGCAGTGTGTCACGACTTGGA CCTGTCCAAATTTGTGCTGTTGAAGAGAAACCTGGAGCTCTGCTGGTCCATTGGCAGCAGGTAGAGACA GAGAAACCAGCAGAAATTGGCTCATTCAGGCTACAACGAGCATACGGAGATGCACGTGGCCGTCGTGA GTTAGAAGCCAATTTCCACGATGAGTATGTGGGACCTGAATGTCACCACCTTATACGCAATCTGCGACCA AAGGAACCAGTCACTCTGCGAGTCTGTTGCCGTGAAGACAGTGAGCTTGCACCGTGGAGTACTTGGTCT GTGTTACATGTTGCTGCAACCTCACTACCTCCATTCTGCTGGGAGGGAACAAATCAGAACTACACTATAA CAAATGAGAAACAACTGGCCACAAAAAGCACCGCAGAAACGTCTGTCCTTTTTTCATGTGGGCCACAATT TGGACCAGGACATGCAGTGGAATTCACTGTTCTAGAATGTGGTGCAGGATGTAGTGACGAAGGTCTGGC TCTTGCCGATCACCATTTTAGTGATGAAAATCTTCTCCAGCCTGGGGTAATATTCGTTAATGCACAAGGAA GTGTGTTTGTGGATGGCAAGGGAAAGACTACCAAATTACCTCCCTTAGAAAAAGGCTCAAAACTATGTTT CACTTGTGAACATGTCCGCAGTAGTAAGGTCCGTATCCACATTGACAGTGGTAATAAGACTGTAGCGTAT GACTGGAATGTTAGTAGTCCACTGCAAAAACTATTTTTTGCTATCTCTTTTGGACAAGTTGGCTGGAAGGT GTTGGTTGAATAA

\section{RNAi Lm-crlf3 fragment 1}

TCTTGGAAGGTTGCCAGCAGTCCCAAATTTGGAAGATATTCCTGGTGTGCAGTTTGAATGCTGTCTACAT GCCATTGAAGCTGACTTGGTTCATGCTGTTGAGAGGGTTGGCAGTGTGTCACGACTTGGACCTGTCCAAA TTTGTGCTGTTGAAGAGAAACCTGGAGCTCTGCTGGTCCATTGGCAGCAGGTAGAGACAGAGAAACCAG CAGAAATTGGCTCATTCAGGCTACAACGAGCATACGGAGATGCACGTGGCCGTCGTGAGTTAGAAGCCA ATTTCCACGATGAGTATGTGGGACCTGAATGTCACCACCTTATACG

\section{RNAi $\mathbf{L m}$-crlf3 fragment 2}

GGAACCAGTCACTCTGCGAGTCTGTTGCCGTGAAGACAGTGAGCTTGCACCGTGGAGTACTTGGTCTGT GTTACATGTTGCTGCAACCTCACTACCTCCATTCTGCTGGGAGGGAACAAATCAGAACTACACTATAACA AATGAGAAACAACTGGCCACAAAAAGCACCGCAGAAACGTCTGTCCTTTTTTCATGTGGGCCACAATTTG GACCAGGACATGCAGTGGAATTCACTGTTCTAGAATGTGGTGCAGGATGTAGTGACGAAGGTCTGGCTC TTGCCGATCACCATTTTAGTGATGAAAATCTTCTCCAGCCTGGGGTAATATTCG

\section{Lm-CRLF3}

MEQSKSNSVFVEAVDAAEEYVKKLEDLLKTLTEAEHQVKHSAKQARADVNFALDTLAATFTNALKRRRSMLL EEVDRLCSEGLEPLIECRDLITTQMQVAHSYANEGRHSLNGSNKCPVAESWDDYTHSYAECGAAFLGRLPAV PNLEDIPGVQFECCLHAIEADLVHAVERVGSVSRLGPVQICAVEEKPGALLVHWQQVETEKPAEIGSFRLQRA YGDARGRRELEANFHDEYVGPECHHLIRNLRPKEPVTLRVCCREDSELAPWSTWSVLHVAATSLPPFCWEGT NQNYTITNEKQLATKSTAETSVLFSCGPQFGPGHAVEFTVLECGAGCSDEGLALADHHFSDENLLQPGVIFVN AQGSVFVDGKGKTTKLPPLEKGSKLCFTCEHVRSSKVRIHIDSGNKTVAYDWNVSSPLQKLFFAISFGQVGWK VLVE

\section{Lm-rpt3 (Accession Number MN245517)}

CDSTTGGGGATCGGTGCGTCAGATGATACAGATTCCGAAGATTTGTACACAAAGTATAAGAAACTTCAG AGGCAGCTAGAATTCTTAGCTGTGCAGGAAGAATACATTAAGGATGAGCAGCGCAATTTGAAAAAGGA ATACCTGCACGCTCAGGAGGAAGTTAAGCGGATACAGAGTGTCCCCCTGGTTATCGGGCAGTTCCTCGA AGCTGTTGACCAGAATACTGGAATAGTTGGCAGCACTACAGGTTCAAACTATTATGTCAGAATACTCTCA 
ACAATTGACCGGGAACTTCTGAAACCTTCTGCAAGTGTTGCCCTTCATAAACACAGCAATGCACTGGTTG ATGTTCTTCCACCTGAGGCTGATTCATCTATATCAATGCTTCAGGCAGATGAAAAGCCAGATGTGACATA CTCAGACATTGGAGGTATGGACATGCAGAAGCAAGAAATTCGTGAGGCTGTGGAGTTACCCTTGACACA CTTCGAACTGTACAAACAGATCGGTATAGACCCCCCACGAGGTGTGCTGATGTATGGGCCACCAGGCTG TGGAAAGACCATGCTGGCAAAGGCTGTCGCTCACCATACTACAGCGGCTTTCATCCGTGTTGTCGGCTCA GAGTTTGTGCAGAAATATTTAGGAGAAGGTCCAAGAATGGTGAGAGATGTTTTCAGACTTGCCAAGGAA AACTCTCCCGCCATCATATTCATTGATGAGATTGATGCCATAGCAACAAAGCGATTTGATGCCCAGACTG GTGCAGACAGAGAAGTGCAAAGAATATTGTTAGAATTGCTCAACCAAATGGATGGCTTCGATCAAACAA CAAATGTTAAGGTGATAATGGCAACGAATCGTGCAGACACTCTTGACCCTGCACTACTCAGGCCCGGCC GTCTTGATCGTAAGATCGAATTTCCTTTACCTGACCGGAGGCAAAAGCGGTTGATTTTCTCAACCATTACA AGCAAGATGAACCTGAGTGAAGAAGTGGACCTTGAGGATTATGTTGCTCGTCCAGATCGCATCTCTGGT GCAGACATCAATGCCATCTGTCAGGAGGCTGGAATGCATGCAGTACGTGAGAACCGCTACATAGTACTT ACAAAAGATTTCGAAAAGGGATACAAAAATAATATAAAGAAGGATGAATCAGAGCATGAATTCTATAAA TAA

\section{RNAi Lm-rpt3 fragment}

GATGAGCAGCGCAATTTGAAAAAGGAATACCTGCACGCTCAGGAGGAAGTTAAGCGGATACAGAGTGT CCCCCTGGTTATCGGGCAGTTCCTCGAAGCTGTTGACCAGAATACTGGAATAGTTGGCAGCACTACAGGT TCAAACTATTATGTCAGAATACTCTCAACAATTGACCGGGAACTTCTGAAACCTTCTGCAAGTGTTGCCCT TCATAAACACAGCAATGCACTGGTTGATGTTCTTCCACCTGAGGCTGATTCATCTATATCAATGCTTCAGG CAGATGAAAAGCCAGATGTG

\section{Lm-RPT3}

MGIGASDDTDSEDLYTKYKKLQRQLEFLAVQEEYIKDEQRNLKKEYLHAQEEVKRIQSVPLVIGQFLEAVDQNT GIVGSTTGSNYYVRILSTIDRELLKPSASVALHKHSNALVDVLPPEADSSISMLQADEKPDVTYSDIGGMDMQK QEIREAVELPLTHFELYKQIGIDPPRGVLMYGPPGCGKTMLAKAVAHHTTAAFIRVVGSEFVQKYLGEGPRMV RDVFRLAKENSPAIIFIDEIDAIATKRFDAQTGADREVQRILLELLNQMDGFDQTTNVKVIMATNRADTLDPAL LRPGRLDRKIEFPLPDRRQKRLIFSTITSKMNLSEEVDLEDYVARPDRISGADINAICQEAGMHAVRENRYIVLT KDFEKGYKNNIKKDESEHEFYK 


\section{Supplementary File S2}

\begin{tabular}{|c|c|}
\hline Accession & Organism \\
\hline XP_009072478.1 & Acanthisitta chloris \\
\hline XP_022064964.1 & Acanthochromis polyacanthus \\
\hline XP_014938917.1 & Acinonyx jubatus \\
\hline XP_019872756.1 & Aethina tumida \\
\hline XP_018321267.1 & Agrilus planipennis \\
\hline XP_011228679.1 & Ailuropoda melanoleuca \\
\hline XP_006271286.1 & Alligator mississippiensis \\
\hline XP_025052023.1 & Alligator sinensis \\
\hline KQK79452.1 & Amazona aestiva \\
\hline XP_023137832.1 & Amphiprion ocellaris \\
\hline XP_026209971.1 & Anabas testudineus \\
\hline XP_005012328.1 & Anas platyrhynchos \\
\hline XP_008112591.1 & Anolis carolinensis \\
\hline XP_018580096.1 & Anoplophora glabripennis \\
\hline XP_013034377.1 & Anser cygnoides domesticus \\
\hline KFZ46915.1 & Antrostomus carolinensis \\
\hline XP_012304699.1 & Aotus nancymaae \\
\hline KFP90043.1 & Apaloderma vittatum \\
\hline XP_005101005.1 & Aplysia californica \\
\hline XP_019329786.1 & Aptenodytes forsteri \\
\hline XP_013808512.1 & Apteryx australis mantelli \\
\hline XP_025923247.1 & Apteryx rowi \\
\hline XP_011585313.1 & Aquila chrysaetos canadensis \\
\hline XP_022519115.1 & Astyanax mexicanus \\
\hline XP_013855159.1 & Austrofundulus limnaeus \\
\hline XP_007190395.1 & Balaenoptera acutorostrata scammoni \\
\hline XP_010307084.1 & Balearica regulorum gibbericeps \\
\hline POI35415.1 & Bambusicola thoracicus \\
\hline XP_013065600.1 & Biomphalaria glabrata \\
\hline XP_010860000.1 & Bison bison bison \\
\hline PSN57173.1 & Blattella germanica \\
\hline XP_020781407.1 & Boleophthalmus pectinirostris \\
\hline XP_019837514.1 & Bos indicus \\
\hline XP_005897076.1 & Bos mutus \\
\hline NP_001179579.1 & Bos taurus \\
\hline XP_019616751.1 & Branchiostoma belcheri \\
\hline XP_002607148.1 & Branchiostoma floridae \\
\hline XP_006059457.1 & Bubalus bubalis \\
\hline XP_010141264.1 & Buceros rhinoceros silvestris \\
\hline XP_014807689.1 & Calidris pugnax \\
\hline OXB64848.1 & Callipepla squamata \\
\hline XP_002748249.1 & Callithrix jacchus \\
\hline XP_007886607.1 & Callorhinchus milii \\
\hline XP_025706828.1 & Callorhinus ursinus \\
\hline XP_008492870.1 & Calypte anna \\
\hline XP_010956647.1 & Camelus bactrianus \\
\hline
\end{tabular}




\begin{tabular}{|c|c|}
\hline EQB78362.1 & Camelus ferus \\
\hline XP_005624890.1 & Canis lupus familiaris \\
\hline ELT93286.1 & Capitella teleta \\
\hline XP_017919752.1 & Capra hircus \\
\hline XP_026064468.1 & Carassius auratus \\
\hline XP_009694409.1 & Cariama cristata \\
\hline XP_021566483.1 & Carlito syrichta \\
\hline XP_020030060.1 & Castor canadensis \\
\hline KFP53478.1 & Cathartes aura \\
\hline XP_003469539.1 & Cavia porcellus \\
\hline XP_017397505.1 & Cebus capucinus imitator \\
\hline XP_023235648.1 & Centruroides sculpturatus \\
\hline XP_004441962.1 & Ceratotherium simum simum \\
\hline XP_011923235.1 & Cercocebus atys \\
\hline OWK14423.1 & Cervus elaphus hippelaphus \\
\hline XP_010001080.1 & Chaetura pelagica \\
\hline XP_009878545.1 & Charadrius vociferus \\
\hline XP_007055198.1 & Chelonia mydas \\
\hline XP_013358923.1 & Chinchilla lanigera \\
\hline XP_010129032.1 & Chlamydotis macqueenii \\
\hline XP_008011601.1 & Chlorocebus sabaeus \\
\hline XP_005282885.1 & Chrysemys picta bellii \\
\hline XP_006832359.1 & Chrysochloris asiatica \\
\hline NP_001107600.1 & Ciona intestinalis \\
\hline XP_012681412.1 & Clupea harengus \\
\hline OXB77036.1 & Colinus virginianus \\
\hline XP_010202715.1 & Colius striatus \\
\hline XP_011793088.1 & Colobus angolensis palliatus \\
\hline PKK25624.1 & Columba livia \\
\hline XP_004684934.1 & Condylura cristata \\
\hline XP_008635809.1 & Corvus brachyrhynchos \\
\hline XP_010405723.1 & Corvus cornix cornix \\
\hline XP_015735107.1 & Coturnix japonica \\
\hline EKC31545.1 & Crassostrea gigas \\
\hline XP_022315438.1 & Crassostrea virginica \\
\hline ERE67541.1 & Cricetulus griseus \\
\hline XP_019397971.1 & Crocodylus porosus \\
\hline XP_023714279.1 & Cryptotermes secundus \\
\hline XP_009562302.1 & Cuculus canorus \\
\hline XP_024920273.1 & Cynoglossus semilaevis \\
\hline XP_015234911.1 & Cyprinodon variegatus \\
\hline XP_018931037.1 & Cyprinus carpio \\
\hline NP_001017817.2 & Danio rerio \\
\hline KZS07498.1 & Daphnia magna \\
\hline XP_004454934.1 & Dasypus novemcinctus \\
\hline XP_022451607.1 & Delphinapterus leucas \\
\hline XP_019772603.1 & Dendroctonus ponderosae \\
\hline XP_024420759.1 & Desmodus rotundus \\
\hline XP_012872027.1 & Dipodomys ordii \\
\hline XP_025954764.1 & Dromaius novaehollandiae \\
\hline
\end{tabular}




\begin{tabular}{|c|c|}
\hline XP_004707397.1 & Echinops telfairi \\
\hline XP_009634329.1 & Egretta garzetta \\
\hline XP_006890996.1 & Elephantulus edwardii \\
\hline XP_008146001.1 & Eptesicus fuscus \\
\hline XP_014717524.1 & Equus asinus \\
\hline XP_023508816.1 & Equus caballus \\
\hline XP_008519700.1 & Equus przewalskii \\
\hline XP_007530877.1 & Erinaceus europaeus \\
\hline XP_010904498.1 & Esox lucius \\
\hline XP_010154375.1 & Eurypyga helias \\
\hline XP_020899993.1 & Exaiptasia pallida \\
\hline XP_013160854.1 & Falco peregrinus \\
\hline XP_003996581.1 & Felis catus \\
\hline XP_016158315.1 & Ficedula albicollis \\
\hline XP_011299889.1 & Fopius arisanus \\
\hline XP_010640722.1 & Fukomys damarensis \\
\hline KFW06266.1 & Fulmarus glacialis \\
\hline XP_012710358.1 & Fundulus heteroclitus \\
\hline XP_008583735.1 & Galeopterus variegatus \\
\hline NP_001073187.2 & Gallus gallus \\
\hline PWA16083.1 & Gambusia affinis \\
\hline XP_009811178.1 & Gavia stellata \\
\hline XP_019370646.1 & Gavialis gangeticus \\
\hline XP_015280181.1 & Gekko japonicus \\
\hline XP_014164411.1 & Geospiza fortis \\
\hline isotig00932 & Gryllus bimaculatus \\
\hline KFQ03222.1 & Haliaeetus albicilla \\
\hline XP_010577044.1 & Haliaeetus leucocephalus \\
\hline XP_004870743.1 & Heterocephalus glaber \\
\hline XP_019745470.1 & Hippocampus comes \\
\hline XP_019502493.1 & Hipposideros armiger \\
\hline NP_057070.3 & Homo sapiens \\
\hline XP_002158017.2 & Hydra vulgaris \\
\hline XP_017347736.1 & Ictalurus punctatus \\
\hline XP_005327851.1 & Ictidomys tridecemlineatus \\
\hline XP_012805063.1 & Jaculus jaculus \\
\hline XP_017260803.2 & Kryptolebias marmoratus \\
\hline XP_020502913.1 & Labrus bergylta \\
\hline XP_010734755.1 & Larimichthys crocea \\
\hline XP_018540171.1 & Lates calcarifer \\
\hline XP_005993818.1 & Latimeria chalumnae \\
\hline XP_017667640.1 & Lepidothrix coronata \\
\hline XP_006635289.1 & Lepisosteus oculatus \\
\hline XP_023020995.1 & Leptinotarsa decemlineata \\
\hline XP_006739091.1 & Leptonychotes weddellii \\
\hline KFQ15088.1 & Leptosomus discolor \\
\hline PKU34418.1 & Limosa lapponica baueri \\
\hline XP_013780331.1 & Limulus polyphemus \\
\hline XP_007452445.1 & Lipotes vexillifer \\
\hline This publication & Locusta migratoria \\
\hline
\end{tabular}




\begin{tabular}{|c|c|}
\hline XP_021393443.1 & Lonchura striata domestica \\
\hline XP_009067299.1 & Lottia gigantea \\
\hline XP_023409143.1 & Loxodonta africana \\
\hline NP_001244548.1 & Macaca mulatta \\
\hline XP_011745721.1 & Macaca nemestrina \\
\hline XP_017928523.1 & Manacus vitellinus \\
\hline XP_011848495.1 & Mandrillus leucophaeus \\
\hline XP_017519933.1 & Manis javanica \\
\hline XP_015356650.1 & Marmota marmota marmota \\
\hline XP_026180724.1 & Mastacembelus armatus \\
\hline XP_004556457.1 & Maylandia zebra \\
\hline XP_010719868.1 & Meleagris gallopavo \\
\hline XP_005146241.1 & Melopsittacus undulatus \\
\hline XP_021486640.1 & Meriones unguiculatus \\
\hline KFQ25580.1 & Merops nubicus \\
\hline KFQ22702.1 & Mesitornis unicolor \\
\hline XP_005077041.1 & Mesocricetus auratus \\
\hline XP_012593827.1 & Microcebus murinus \\
\hline XP_005350307.1 & Microtus ochrogaster \\
\hline XP_016063084.1 & Miniopterus natalensis \\
\hline XP_021346829.1 & Mizuhopecten yessoensis \\
\hline XP_001368350.1 & Monodelphis domestica \\
\hline XP_020460510.1 & Monopterus albus \\
\hline XP_021032210.1 & Mus caroli \\
\hline AAD31759.1 & Mus musculus \\
\hline XP_021068474.1 & Mus pahari \\
\hline XP_004747109.1 & Mustela putorius furo \\
\hline EPQ14786.1 & Myotis brandtii \\
\hline XP_015419621.1 & Myotis davidii \\
\hline XP_014322915.1 & Myotis lucifugus \\
\hline XP_008819846.1 & Nannospalax galili \\
\hline XP_018430334.1 & Nanorana parkeri \\
\hline XP_001629022.1 & Nematostella vectensis \\
\hline XP_006801999.1 & Neolamprologus brichardi \\
\hline XP_021560265.1 & Neomonachus schauinslandi \\
\hline XP_024592629.1 & Neophocaena asiaeorientalis asiaeorientalis \\
\hline OBS69124.1 & Neotoma lepida \\
\hline KFQ53661.1 & Nestor notabilis \\
\hline XP_017771049.1 & Nicrophorus vespilloides \\
\hline XP_022207526.1 & Nilaparvata lugens \\
\hline XP_009465290.1 & Nipponia nippon \\
\hline XP_015797750.1 & Nothobranchius furzeri \\
\hline XP_025902638.1 & Nothoprocta perdicaria \\
\hline XP_010775375.1 & Notothenia coriiceps \\
\hline XP_021270940.1 & Numida meleagris \\
\hline XP_004593943.1 & Ochotona princeps \\
\hline XP_023575619.1 & Octodon degus \\
\hline XP_014770014.1 & Octopus bimaculoides \\
\hline XP_004399677.1 & Odobenus rosmarus divergens \\
\hline XP_020754047.1 & Odocoileus virginianus texanus \\
\hline
\end{tabular}




\begin{tabular}{|c|c|}
\hline XP_020324387.1 & Oncorhynchus kisutch \\
\hline XP_021481469.1 & Oncorhynchus mykiss \\
\hline XP_024286146.1 & Oncorhynchus tshawytscha \\
\hline XP_009936722.1 & Opisthocomus hoazin \\
\hline XP_020605374.1 & Orbicella faveolata \\
\hline XP_004267215.1 & Orcinus orca \\
\hline XP_005469141.1 & Oreochromis niloticus \\
\hline XP_001511274.1 & Ornithorhynchus anatinus \\
\hline XP_007935528.1 & Orycteropus afer afer \\
\hline KRT80600.1 & Oryctes borbonicus \\
\hline XP_017204621.1 & Oryctolagus cuniculus \\
\hline XP_023813029.1 & Oryzias latipes \\
\hline XP_024127665.1 & Oryzias melastigma \\
\hline XP_003797224.1 & Otolemur garnettii \\
\hline XP_014954004.1 & Ovis aries \\
\hline XP_011970064.1 & Ovis aries musimon \\
\hline XP_003812023.1 & Pan paniscus \\
\hline PNI47164.1 & Pan troglodytes \\
\hline XP_019279402.1 & Panthera pardus \\
\hline XP_007076253.1 & Panthera tigris altaica \\
\hline XP_005983197.1 & Pantholops hodgsonii \\
\hline XP_019950200.1 & Paralichthys olivaceus \\
\hline XP_023647799.1 & Paramormyrops kingsleyae \\
\hline XP_015501248.1 & Parus major \\
\hline OPJ88558.1 & Patagioenas fasciata monilis \\
\hline KFQ54107.1 & Pelecanus crispus \\
\hline XP_006125219.2 & Pelodiscus sinensis \\
\hline XP_006977564.1 & Peromyscus maniculatus bairdii \\
\hline KFQ75603.1 & Phaethon lepturus \\
\hline KFW93159.1 & Phalacrocorax carbo \\
\hline XP_020818920.1 & Phascolarctos cinereus \\
\hline KFQ87492.1 & Phoenicopterus ruber ruber \\
\hline XP_009909841.1 & Picoides pubescens \\
\hline XP_023038667.1 & Piliocolobus tephrosceles \\
\hline KFZ55261.1 & Podiceps cristatus \\
\hline XP_007565080.1 & Poecilia formosa \\
\hline XP_014838175.1 & Poecilia mexicana \\
\hline XP_008414767.1 & Poecilia reticulata \\
\hline XP_020639319.1 & Pogona vitticeps \\
\hline XP_025081884.1 & Pomacea canaliculata \\
\hline NP_001125166.1 & Pongo abelii \\
\hline XP_014664328.1 & Priapulus caudatus \\
\hline XP_012503685.1 & Propithecus coquereli \\
\hline XP_015676750.1 & Protobothrops mucrosquamatus \\
\hline XP_005526750.1 & Pseudopodoces humilis \\
\hline XP_010080323.1 & Pterocles gutturalis \\
\hline XP_006925041.1 & Pteropus alecto \\
\hline XP_025774026.1 & Puma concolor \\
\hline XP_005739982.1 & Pundamilia nyererei \\
\hline XP 017569244.1 & Pygocentrus nattereri \\
\hline
\end{tabular}




\begin{tabular}{|c|c|}
\hline XP_009322062.1 & Pygoscelis adeliae \\
\hline XP_007423555.1 & Python bivittatus \\
\hline PIO14754.1 & Rana catesbeiana \\
\hline NP_001162083.1 & Rattus norvegicus \\
\hline XP_020384420.1 & Rhincodon typus \\
\hline XP_019606266.1 & Rhinolophus sinicus \\
\hline XP_017746352.1 & Rhinopithecus bieti \\
\hline XP_010371287.1 & Rhinopithecus roxellana \\
\hline XP_016007986.1 & Rousettus aegyptiacus \\
\hline XP_003931522.1 & Saimiri boliviensis boliviensis \\
\hline NP_001167401.1 & Salmo salar \\
\hline XP_023359382.1 & Sarcophilus harrisii \\
\hline XP_018617526.1 & Scleropages formosus \\
\hline AWP19462.1 & Scophthalmus maximus \\
\hline XP_009092824.1 & Serinus canaria \\
\hline XP_022595132.1 & Seriola dumerili \\
\hline XP_023269631.1 & Seriola lalandi dorsalis \\
\hline XP_016297041.1 & Sinocyclocheilus anshuiensis \\
\hline XP_016148870.1 & Sinocyclocheilus grahami \\
\hline XP_016383026.1 & Sinocyclocheilus rhinocerous \\
\hline XP_012788203.1 & Sorex araneus \\
\hline XP_008304604.1 & Stegastes partitus \\
\hline XP_009677349.1 & Struthio camelus australis \\
\hline XP_014733689.1 & Sturnus vulgaris \\
\hline XP_022782357.1 & Stylophora pistillata \\
\hline NP_001231258.1 & Sus scrofa \\
\hline XP_002195017.1 & Taeniopygia guttata \\
\hline XP_003965013.1 & Takifugu rubripes \\
\hline XP_009991254.1 & Tauraco erythrolophus \\
\hline XP_024048299.1 & Terrapene mexicana triunguis \\
\hline AAR25665.1 & Tetraodon nigroviridis \\
\hline XP_013921879.1 & Thamnophis sirtalis \\
\hline XP_025217567.1 & Theropithecus gelada \\
\hline XP_010216717.1 & Tinamus guttatus \\
\hline XP_008190449.1 & Tribolium castaneum \\
\hline XP_004385452.1 & Trichechus manatus latirostris \\
\hline XP_014445594.1 & Tupaia chinensis \\
\hline XP_009970055.1 & Tyto alba \\
\hline XP_026244746.1 & Urocitellus parryii \\
\hline XP_008693592.1 & Ursus maritimus \\
\hline XP_006212079.2 & Vicugna pacos \\
\hline XP_025871908.1 & Vulpes vulpes \\
\hline NP_001080600.1 & Xenopus laevis \\
\hline XP_002943331.1 & Xenopus tropicalis \\
\hline XP_005803247.1 & Xiphophorus maculatus \\
\hline XP_014124947.1 & Zonotrichia albicollis \\
\hline XP_021931883.1 & Zootermopsis nevadensis \\
\hline
\end{tabular}




\section{Supplementary File S3}

\#NEXUS

begin trees;

tree tree_ $1=[\& R]$

(((Branchiostoma_belcheri:0.012325,Branchiostoma_floridae:0.017614):0.90175,((Ciona_intestinalis:1.2 81126,(Hydra_vulgaris:1.263472,(Exaiptasia_pallida:0.254021,(Nematostella_vectensis:0.2153,(Orbicella _faveolata:0.066114,Stylophora_pistillata:0.073016):0.282425):0.05872):0.369087):0.841556):0.1096,(P riapulus_caudatus:1.624847,((Capitella_teleta:0.907863,((Octopus_bimaculoides:0.712251,(Mizuhopect en_yessoensis:0.448395,(Crassostrea_gigas:0.065224,Crassostrea_virginica:0.059234):0.399309):0.0860 22):0.067423,(Lottia_gigantea:0.429117,(Pomacea_canaliculata:0.497819,(Biomphalaria_glabrata:0.174 205,Aplysia_californica:0.297839):0.330974):0.217572):0.081558):0.260215):0.192004,((Centruroides_sc ulpturatus:0.703397,Limulus_polyphemus:0.773795):0.444865,(Daphnia_magna:3.622483,((Fopius_aris anus:1.77393,(Nilaparvata_lugens:0.836626,(Locusta_migratoria:0.666096,(Gryllus_bimaculatus:0.30287 7,(Blattella_germanica:0.199136,(Zootermopsis_nevadensis:0.088772,Cryptotermes_secundus:0.123073 ):0.105089):0.119861):0.068588):0.083773):0.315619):0.225781,(Agrilus_planipennis:2.024432,(Nicroph orus_vespilloides:0.809282,(Oryctes_borbonicus:0.680904,((Dendroctonus_ponderosae:1.333375,Triboli um_castaneum:0.407003):0.110895,(Aethina_tumida:0.444539,(Leptinotarsa_decemlineata:0.355535,A noplophora_glabripennis:0.239411):0.168455):0.075996):0.195875):0.077035):0.206519):0.35103):0.21 462):0.165737):0.182164):0.065355):0.110206):0.147376):0.420103,((Rhincodon_typus:0.109472,Callor hinchus_milii:0.116643):0.091124,((Latimeria_chalumnae:0.111016,(Lepisosteus_oculatus:0.116819,((Sc leropages_formosus:0.048942,Paramormyrops_kingsleyae:0.062441):0.045352,((Clupea_harengus:0.07 2744,((Ictalurus_punctatus:0.118874,(Astyanax_mexicanus:0.022294,Pygocentrus_nattereri:0.029187):0 .022166):0.052638,(Danio_rerio:0.029244,(Sinocyclocheilus_rhinocerous:0.018516,((Carassius_auratus:0 .011403,Cyprinus_carpio:0.018447):0.002071,(Sinocyclocheilus_grahami:2.0E-

6,Sinocyclocheilus_anshuiensis:0.00674):0.004504):0.004916):0.010362):0.05438):0.022335):0.020085,(( Esox_lucius:0.045674,(Oncorhynchus_tshawytscha:0.202226,(Salmo_salar:0.003809,(Oncorhynchus_my kiss:2.0E-6,Oncorhynchus_kisutch:3.0E-

6):0.044675):0.003784):0.025983):0.046155,(Boleophthalmus_pectinirostris:0.146015,(Hippocampus_co mes:0.164321,((Takifugu_rubripes:0.04048,Tetraodon_nigroviridis:0.051786):0.056078,((Larimichthys_c rocea:0.011819,Labrus_bergylta:0.046002):0.005957,(Notothenia_coriiceps:0.086417,(((Seriola_lalandi_ dorsalis:2.0E-

6,Seriola_dumerili:0.002274):0.014092,((Monopterus_albus:0.045216,(Anabas_testudineus:0.042363,M astacembelus_armatus:0.039158):0.005114):0.004518,(Lates_calcarifer:0.010767,(Scophthalmus_maxi mus:0.061769,(Cynoglossus_semilaevis:0.407866,Paralichthys_olivaceus:0.035753):9.88E-

4):0.012651):0.003041):0.004663):0.007692,((Stegastes_partitus:0.006508,(Amphiprion_ocellaris:0.0072 17,Acanthochromis_polyacanthus:0.006553):0.009914):0.027157,((Nothobranchius_furzeri:0.064303,(A ustrofundulus_limnaeus:0.042888,Kryptolebias_marmoratus:0.041922):0.037314):0.006983,(((Oryzias_ melastigma:0.04168,Oryzias_latipes:0.049342):0.057216,(Neolamprologus_brichardi:0.002271,(Pundami lia_nyererei:2.0E-6,(Maylandia_zebra:0.002272,Oreochromis_niloticus:0.002271):2.0E-6):2.0E-

6):0.02886):0.014078,(Fundulus_heteroclitus:0.030712,(Cyprinodon_variegatus:0.021095,((Poecilia_for mosa:0.004626,Poecilia_mexicana:0.002307):0.002329,(Poecilia_reticulata:0.032181,(Xiphophorus_mac 
ulatus:0.007016,Gambusia_affinis:0.004678):0.002347):0.002235):0.022953):0.002805):0.022665):0.002 196):0.010903):0.017889):0.005173):0.00324):0.015033):0.005856):0.015233):0.060883):0.045891):0.03 6556):0.080159):0.155826):0.022579,(((Rana_catesbeiana:1.051232,Nanorana_parkeri:0.105402):0.075 603,(Xenopus_laevis:0.04921,Xenopus_tropicalis:0.067101):0.148951):0.110001,(((Gekko_japonicus:0.0 59287,((Anolis_carolinensis:0.202053,Pogona_vitticeps:0.071433):0.006445,(Python_bivittatus:0.015711 ,(Protobothrops_mucrosquamatus:0.024825,Thamnophis_sirtalis:0.032773):0.034477):0.061864):0.0191 67):0.0486,((Pelodiscus_sinensis:0.046733,(Chelonia_mydas:0.012502,(Chrysemys_picta_bellii:0.005691, Terrapene_mexicana_triunguis:0.063563):0.011571):0.011074):0.052509,(((Alligator_sinensis:0.090794, Alligator_mississippiensis:0.002836):0.004289,(Crocodylus_porosus:0.008234,Gavialis_gangeticus:0.026 15):0.01096):0.052136,(Acanthisitta_chloris:2.0E-6,(((Corvus_brachyrhynchos:2.0E-

6,Corvus_cornix_cornix:3.0E-6):0.003022,((Parus_major:0.002352,Pseudopodoces_humilis:2.0E-

6):0.004918,((Sturnus_vulgaris:0.059037,Ficedula_albicollis:0.002219):0.061663,((Lepidothrix_coronata: 2.0E-6,Manacus_vitellinus:0.102002):0.034087,(Taeniopygia_guttata:3.0E-

6,(Lonchura_striata_domestica:0.002232,(Zonotrichia_albicollis:2.0E-

6,(Serinus_canaria:0.076226,Geospiza_fortis:0.064365):0.067466):0.037126):0.004927):0.007494):0.004 819):0.004647):0.008143):0.010115,(((Limosa_lapponica_baueri:0.017919,Calidris_pugnax:0.006517):0. 010765,(Amazona_aestiva:0.003979,(Nestor_notabilis:0.01794,Melopsittacus_undulatus:0.099714):2.0E

6):0.023293):0.003372,((Patagioenas_fasciata_monilis:0.022383,((Coturnix_japonica:0.010972,Numida_ meleagris:0.018713):0.004038,(Meleagris_gallopavo:0.006557,(Gallus_gallus:0.011826,(Bambusicola_th oracicus:0.045424,(Colinus_virginianus:0.020823,Callipepla_squamata:2.0E-6):0.03514):0.036083):3.0E6):0.002274):0.021859):0.004066,((Antrostomus_carolinensis:0.023943,Nipponia_nippon:0.002996):0.0 02826,((Pygoscelis_adeliae:2.0E-6,Aptenodytes_forsteri:2.0E-

6):0.005932,((Calypte_anna:0.033423,(Haliaeetus_albicilla:2.0E-6,(Aquila_chrysaetos_canadensis:2.0E-

6,(Haliaeetus_leucocephalus:3.0E-6,Egretta_garzetta:0.005926):3.0E-6):3.0E-

6):0.021893):0.022221,(Podiceps_cristatus:0.021096,((Merops_nubicus:0.042599,(Anas_platyrhynchos:0 .001918,Anser_cygnoides_domesticus:0.010421):0.014031):0.013191,((Phoenicopterus_ruber_ruber:3.0 $\mathrm{E}-$

6,(((Charadrius_vociferus:0.008922,Opisthocomus_hoazin:0.008968):0.002933,(Colius_striatus:0.058057 ,(Picoides_pubescens:0.033135,Tyto_alba:0.006703):0.005319):2.0E-6):0.010966,(Cathartes_aura:2.0E-

6,((Eurypyga_helias:0.076989,Columba_livia:0.085542):0.020338,(Fulmarus_glacialis:3.0E-

6,(Chlamydotis_macqueenii:0.021779,Mesitornis_unicolor:0.022279):0.011055):0.00539):0.013729):0.0 13639):2.77E-

4):0.007953,((Cuculus_canorus:0.034941,((Nothoprocta_perdicaria:0.011423,Tinamus_guttatus:0.00719 7):0.008221,(Struthio_camelus_australis:0.026345,(Dromaius_novaehollandiae:0.008913,(Apteryx_rowi: 2.0E-6,Apteryx_australis_mantelli:2.0E-6):0.005978):0.006039):2.0E-

6):0.04127):0.021095,(((Pterocles_gutturalis:0.025796,Chaetura_pelagica:0.090372):0.026846,(Buceros_ rhinoceros_silvestris:0.055095,(Tauraco_erythrolophus:0.00895,Leptosomus_discolor:0.011861):0.0029 47):3.0E-

6):0.022507,(Pelecanus_crispus:0.007323,(Balearica_regulorum_gibbericeps:0.022092,((Phalacrocorax_c arbo:0.008919,(Phaethon_lepturus:0.014867,Gavia_stellata:0.021541):3.0E-

6):0.010407,(Cariama_cristata:0.049599,(Apaloderma_vittatum:0.021372,Falco_peregrinus:0.037817):0. 005137):0.018166):0.021232):2.26E-4):0.011972):0.017207):0.021657):0.015336):0.013256):3.0E- 
6):3.0E-

6):0.031237):0.044793):0.002458):0.007389):0.009998):0.034278):0.017051):0.014623):0.03395,((Ornit horhynchus_anatinus:0.092318,(Monodelphis_domestica:0.01968,(Phascolarctos_cinereus:0.032329,Sar cophilus_harrisii:0.010005):0.004689):0.016475):0.011127,(Trichechus_manatus_latirostris:0.004814,((O rycteropus_afer_afer:0.023514,(Chrysochloris_asiatica:0.032855,Elephantulus_edwardii:0.025591):0.00 5868):2.0E-

6,(Dasypus_novemcinctus:0.016462,((((Saimiri_boliviensis_boliviensis:0.00231,(Callithrix_jacchus:0.0046 26,Aotus_nancymaae:0.002309):2.0E-6):0.009325,((Homo_sapiens:3.0E-

6,(Pan_troglodytes:0.161172,Pan_paniscus:2.0E-6):2.0E-

6):0.002318,((Cercocebus_atys:0.002332,(Macaca_mulatta:2.0E-

6,(Chlorocebus_sabaeus:0.01019,(Mandrillus_leucophaeus:0.06121,Pongo_abelii:0.08343):0.008844):2. 0E-6):2.0E-6):0.00235,(Piliocolobus_tephrosceles:6.6E-

5,(Colobus_angolensis_palliatus:0.058436,(Rhinopithecus_roxellana:0.054467,(Theropithecus_gelada:0. 042476,(Rhinopithecus_bieti:0.028447,(Macaca_nemestrina:2.0E-

6,Cebus_capucinus_imitator:0.038244):2.0E-6):0.038384):0.019692):0.03554):0.042373):0.004634):2.0E6):0.002295):0.011721,(Galeopterus_variegatus:0.009295,((Microcebus_murinus:0.006993,(Otolemur_g arnettii:0.01173,Propithecus_coquereli:0.018969):2.0E-

6):0.009254,((Carlito_syrichta:0.034788,(Ochotona_princeps:0.056497,Oryctolagus_cuniculus:0.014349) :0.010815):0.003618,((Marmota_marmota_marmota:0.002295,(Urocitellus_parryii:0.145943,Ictidomys_ tridecemlineatus:0.002289):2.0E-

6):0.006952,((Castor_canadensis:0.013852,Dipodomys_ordii:0.02362):0.002513,((Fukomys_damarensis: 0.13093,(Heterocephalus_glaber:0.016987,(Chinchilla_lanigera:0.023157,(Cavia_porcellus:0.014734,Oct odon_degus:0.181631):0.00467):0.002679):0.001304):0.012462,(Nannospalax_galili:0.093691,(Jaculus_j aculus:0.030842,((Rattus_norvegicus:0.023719,(Mus_caroli:3.0E-

6,(AAD31759_AF120152_1_:0.00231,Mus_pahari:0.009209):0.002269):0.017235):0.005529,(Meriones_ unguiculatus:0.01851,((Mesocricetus_auratus:0.006851,Cricetulus_griseus:0.121145):0.004681,(Microtu s_ochrogaster:0.010893,(Peromyscus_maniculatus_bairdii:0.002281,Neotoma_lepida:0.039328):0.0023 16):0.001998):2.0E-

6):0.003032):0.016286):0.007002):0.002352):0.002565):0.0023):0.004624):0.002319):3.0E-

6):0.00229):0.002328,(((Equus_caballus:0.006923,Ceratotherium_simum_simum:0.004673):0.002322,(E ptesicus_fuscus:0.006963,(Pteropus_alecto:0.002304,Rousettus_aegyptiacus:0.011616):0.002331):2.0E6):0.002289,(((Lipotes_vexillifer:0.00229,Orcinus_orca:2.0E-

6):0.00933,(((Cervus_elaphus_hippelaphus:0.327418,Odocoileus_virginianus_texanus:3.0E-

6):0.00686,(Capra_hircus:3.0E-6,Ovis_aries_musimon:0.002287):0.002281):3.0E-

6,(Bubalus_bubalis:3.0E-6,(Bison_bison_bison:0.03545,(Bos_indicus:2.0E-6,Bos_taurus:2.0E-6):2.0E-

6):0.004553):0.002279):0.009324):0.004611,((Erinaceus_europaeus:0.018667,((Sus_scrofa:0.009205,Tup aia_chinensis:0.033709):0.007086,(Condylura_cristata:0.016339,(Hipposideros_armiger:0.00959,Rhinolo phus_sinicus:0.022961):0.002053):0.002203):0.002441):2.0E-6,((Mustela_putorius_furo:2.0E-

6,(Desmodus_rotundus:0.020007,(Felis_catus:2.0E-6,(Puma_concolor:0.002307,(Panthera_pardus:3.0E6,Panthera_tigris_altaica:0.013839):0.002304):3.0E-

6):0.002064):0.007317):0.002312,(Odobenus_rosmarus_divergens:2.0E-

6,(Neomonachus_schauinslandi:0.004629,((Callorhinus_ursinus:0.002316,Leptonychotes_weddellii:0.00 6945):2.0E- 
6,((Vulpes_vulpes:0.017144,Canis_lupus_familiaris:0.027142):0.01037,(((Miniopterus_natalensis:0.0559 54,Balaenoptera_acutorostrata_scammoni:0.023706):0.015316,(Ursus_maritimus:0.041786,(Manis_java nica:0.063135,((Sorex_araneus:0.211952,Bos_mutus:0.279182):0.192784,((Ovis_aries:0.016501,Panthol ops_hodgsonii:0.064817):0.023713,(Equus_asinus:0.022737,Echinops_telfairi:0.0844):0.004711):0.0216 84):0.025608):0.017012):0.02332):0.017161,(Acinonyx_jubatus:0.052102,((Myotis_brandtii:0.020516,(N eophocaena_asiaeorientalis_asiaeorientalis:0.002189,Delphinapterus_leucas:0.002592):0.029592):0.030 901,((Ailuropoda_melanoleuca:0.031971,Equus_przewalskii:0.036145):0.026733,(Myotis_davidii:0.0150 09,(Myotis_lucifugus:0.02029,(Loxodonta_africana:0.06099,(Vicugna_pacos:0.013956,(Camelus_ferus:0. 023109,Camelus_bactrianus:3.0E-

6):0.021151):0.107771):0.089379):0.005269):0.023013):0.019333):0.017988):0.029355):0.031561):0.046 573):0.002304):2.0E-6):2.0E-6):0.004627):0.002308):3.0E-

6):0.004642):0.002332):0.002307):0.002125):0.030504):0.083273):0.048511):0.05116):0.040566):0.4201 03);

end; 


\subsection{Manuscript 3: The human splice variant EV-3 and the Epo-mimetic peptide P16 mediate neuroprotection via the orphan CRLF3 receptor in locusts}

The preliminary manuscript "The human splice variant EV-3 and the Epo-mimetic peptide P16 mediate neuroprotection via the orphan CRLF3 receptor in locusts" is in preparation and will be submitted soon. Potential journals are the current research topic "Cutting-Edge Approaches for CNS Protection and Repair: Focus on Vascular and Degenerative Disorders" edited by Frontiers, Neural Regeneration Research or BMC molecular medicine.

Authors will be: Nina Hahn, Ruoyu Huang, Nicola Schwedhelm-Domeyer, Liane Wüstefeld, Olaf Jahn, Hannelore Ehrenreich and Ralf Heinrich.

The study reported in this manuscript demonstrates the neuroprotective effect of three synthesized Epo-mimetic peptides in a neuroprotection assay using locust primary brain cells. Control peptides that consist of the same amino acids in a different order, did not exhibit any neuroprotective effect. Finally, I showed that the locust ortholog Lm-CRLF3 seems to be crucial for the neuroprotective effect induced by the Epo-mimetic peptide P16 and the endogenous human splice variant EV-3. This final experiment currently consists of three biological replications and additional data are in the progress of being gathered for statistical evaluation. The experimenter is always blinded with respect to the identity of the experimental conditions which ensures the unbiased analysis of the last pending experiments. In conclusion, this study substantiated the hypothesis that human CRLF3 might represent a target for the development of safe, neuroprotective drugs.

My contribution to that manuscript was substantial. The study was designed together with RHe, $\mathrm{HE}$, LW and OJ. I supervised RHu during data collection and was involved in the project management. Furthermore, I did the data analysis as well as visualization and prepared the figures except Figure 5-2 (prepared by RHe). The manuscript was written together with RHe. 


\subsubsection{Abstract}

Erythropoietin (Epo) is a pleiotropic cytokine involved in erythropoiesis and neuroprotection. The Epo-induced signaling pathways of erythropoiesis are well known. On erythroid precursor cells, Epo binds to the homodimeric, classical Epo receptor $\left(\mathrm{EpoR}_{2}\right)$ stimulating signaling pathways that prevent apoptosis. In contrast, the neuroprotective signaling pathways remain largely elusive. Epo might bind to homo-or heterodimeric receptors with or without EpoR. Nevertheless, Epo was used for treatment of stroke in a clinical trial with a negative outcome. The noted severe adverse side effects might have arisen from Epo's ability to stimulate erythropoiesis hindering further clinical studies of Epo application after stroke. Hence, the development of alternative and safe neuroprotective drugs, which selectively stimulate neuroprotection, is required. We tested the Epo-mimetic peptides HBSP, P16 and EMP1 in a neuroprotection assay using primary brain cells from Locusta migratoria. All peptides showed a pronounced neuroprotective effect with P16 being as neuroprotective as Epo. In contrast, control peptides that consist of the identical amino acids in a different order did not show any protective effect. In addition, we tested whether the neuroprotective effects of P16 and the endogenous human splice variant EV-3 were mediated by the orphan cytokine receptor-like factor 3 (CRLF3). Knocking down CRLF3 by soaking RNA interference abolished the neuroprotective effect of P16 and EV-3 demonstrating its necessity. CRLF3 is highly conserved from Cnidaria to Mammalia and emerged together with the origin of the eumetazoan nervous system. Hence, it is likely that human CRLF3 might mediate the neuroprotective effect of P16 and EV-3 as well and should be taken into account for the development of safe, neuroprotective drugs. 


\subsubsection{Introduction}

Erythropoietin (Epo) is a helical glycoprotein named for its role as a hormonal stimulator of erythropoiesis (Erslev, 1953; Jelkmann, 1992). In this function, Epo binds to the homodimeric, „classical“ Epo receptor (EpoR $\left.\mathbf{R}_{\mathbf{2}}\right)$, a cytokine class-1 receptor, expressed on erythroid progenitor cells in the bone marrow. Upon binding, cytosolic parts of $\mathrm{EpoR}_{2}$ are phosphorylated by associated janus kinase 2 (JAK2) and activate intracellular transduction pathways involving signaling transducer and activator of transcription (STAT5), phosphoinositide-3-kinase (PI3/AKT) and mitogen-activated protein kinase (Ras/MAPK). Subsequently, these pathways induce the expression of anti-apoptotic factors that promote erythrocyte differentiation (Cokic et al., 2012; Schmidt et al., 2004; Wandzioch et al., 2004). In addition to its involvement in the hematopoietic system, Epo is also released as a paracrine signal in various tissues to promote cellular survival, to maintain or to re-establish tissue functions (Arcasoy, 2008; Brines and Cerami, 2005; Ghezzi and Brines, 2004). In mammalian nervous systems, Epo is crucial for proper neural development and suppresses neuroinflammatory processes including activation of microglia (Bond and Rex, 2014; Genc et al., 2004; Mitkovski et al., 2015; Yu et al., 2002). It furthermore acts neuroprotectively during ischemic insults (Sirén et al., 2001) as well as during glutamate excitotoxicity (Bernaudin et al., 1999; Morishita et al., 1996) and promotes axonal regeneration (Kretz et al., 2005; Zhang et al., 2009). Neurons, astrocytes, oligodendrocytes, microglia and endothelial cells in the brain express EpoR. Their interaction with Epo has been shown to be crucial for cell survival in the embryonic brain, however homodimeric Epo $R_{2}$ does not account for all Epo-mediated beneficial effects (Chen et al., 2007; Noguchi et al., 2007). Various Epo-like ligands have been described that do not stimulate erythropoiesis (and hence do not activate $E_{p o R_{2}}$ ) but induce protective and regenerative mechanisms in the nervous system. This clearly indicates the presence of alternative neuroprotective Epo-receptors. Potential candidates under investigation are the ephrin B4 receptor (EphB4), the cytokine receptor-like factor 3 (CRLF3) and a heteroreceptor consisting of EpoR and the beta common receptor CD131 (EpoR/ßcR) (reviewed by (Ostrowski and Heinrich, 2018)). Neurons, astrocytes, microglia and vascular cells co-express EpoR and $\beta c R$. Activation of this heteroreceptor mediates Epo-induced neuroprotection or regeneration in primary rat hippocampal neurons, mouse cortical neurons, mouse spinal cord, mouse hippocampus, and human neuroblastoma cells (Brines et al., 2004; Chamorro et al., 2013; Ding et al., 2017; Miller et al., 2015). Cell protection via EpoR/ $\beta c R$ has also been shown in other tissues besides the nervous system including co-transfected human embryonic kidney cells and macrophages (Lee et al., 2015; Miller et al., 2015). However, Epo-induced neuroprotection was also observed in cells not co-expressing EpoR and $\beta c R$ (Nadam et al., 2007; Sanchez et al., 2009; Um et al., 2007). EphB4 is another alternative Epo receptor that is expressed in mammalian neurons and neural stem cells (Ashton et al., 2012; Liu et al., 2017; Pradeep et al., 2015). Signaling of EphB4 and its ligand ephrin-B2 has been associated with 
neural stem cell proliferation, neural lineage induction and synaptic plasticity (Ashton et al., 2012; Henderson et al., 2001; Liu et al., 2017). Studies on various cell types demonstrated that both EphrinB2 and Epo directly activate EphB4 (Pradeep et al., 2015). This suggests that part of the Epomediated beneficial effects in the brain could be due to activation of EphB4.

In previous studies, we have demonstrated that recombinant human Epo and the human splice variant EV-3 protect insect brain neurons in vitro even though insects lack Epo orthologs (Gocht et al., 2009; Hahn et al., 2017; Miljus et al., 2014). Furthermore, EpoR, $\beta c R$ and EphrinB4 orthologs have also not been found in insects. Thus, we examined insect orthologs of CRLF3 as a potential neuroprotective receptor and demonstrated its necessity for Epo-induced neuroprotection in the beetle Tribolium castaneum and the locust Locusta migratoria (Hahn et al., 2017, 2019). CRLF3 is termed as an orphan receptor whose endogenous ligand is still enigmatic. It has been shown that CRLF3-activated signaling pathways include STAT3 activation but are independent of Ras/MAPK (Yang et al., 2009). In locusts, Epo-induced neuroprotection is also dependent on JAK/STAT signaling pathways (Miljus et al., 2014). CRLF3 orthologs are well conserved in Eumetazoa ranging from Cnidaria to Mammalia and emerged with the origin of the eumetazoan nervous system (Hahn et al., 2019). The involvement of insect CRLF3 orthologs in neuroprotection induced by human Epo and the high evolutionary conservation of CRLF3 proteins suggest that Epo might serve as an endogenous ligand for vertebrate CRLF3.

The activation of alternative, neuroprotective Epo receptors by Epo or Epo-like molecules is still elusive. However, this knowledge will be fundamental for the development of safe, neuroprotective drugs not interfering with erythropoietic pathways. To the present, many Epo variants and Epomimetics, which do not stimulate erythropoiesis but are neuroprotective in vertebrates, have been investigated. They include the natural human splice variant EV-3 (Bonnas et al., 2017), peptides mimicking parts of the full length Epo and circularized peptides without any sequence similarity to Epo (Figure 5-1) (sequences are summarized in Table 5-1). The peptide P16 consists of 16 continuous amino acids of Epo's helix A while the helix B surface peptide (HBSP) is related to 11 discontinuous amino acids of Epo's helix B surface (Bonnas, 2009; Brines et al., 2008). In contrast, the cyclic peptide EMP1 consisting of 20 amino acids is not related to the Epo sequence. It mediates neuroprotection but also induces erythropoiesis (Kawakami et al., 2001; Livnah et al., 1996; Wrighton et al., 1996). 


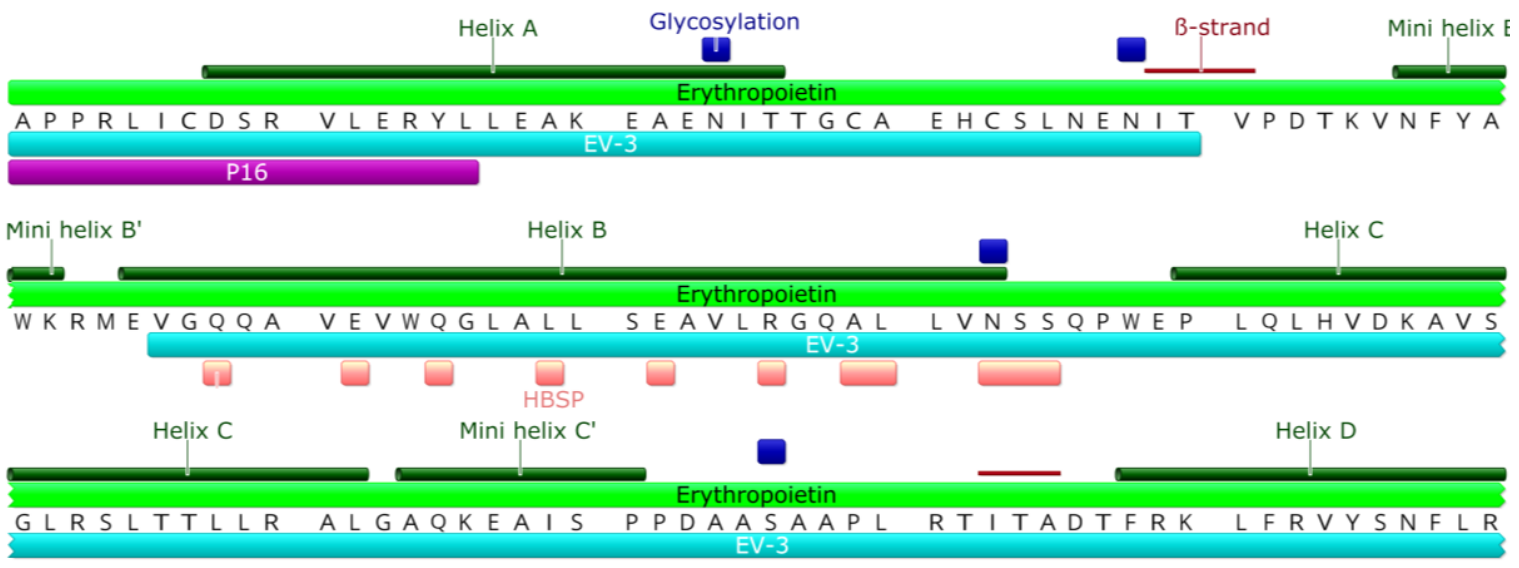

Helix D

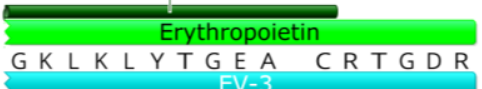

Figure 5-1: Graphic representation of the Epo sequence and corresponding Epo-like molecules. The Epo sequence is depicted in light green. Structural features of Epo are shown above its sequence while Epo-like molecules are shown below. Its four alpha-helices (A-D) are indicated by dark green bars and the $\beta$-strands in brown. Glycosylation sites are marked in blue. The Epo-mimetic peptide P16 is labeled in violet whereas HBSP is labeled in light red. The human splice variant EV-3 lacking exon 3 is represented in light blue.

In this study, we investigated the synthesized neuroprotective Epo-mimetic peptides P16, HBSP, EMP1 and the human splice variant EV-3 for neuroprotective effects on locust primary brain cells in vitro. In addition, we examined whether the neuroprotective effect of EV-3 and P16 is mediated by the locust ortholog Lm-CRLF3 employing soaking RNA interference for receptor knock-down. The results provide first insights into neuroprotective signaling pathways of P16 and EV-3, which are relevant to judge their potential for clinical use. 


\subsubsection{Material and methods}

\subsubsection{Production of Epo-mimetic peptides, Epo and EV-3}

The Epo-mimetic peptides P16 (Bonnas, 2009), HBSP (Brines et al., 2008) and a derivative of EMP1 (Livnah et al., 1996; Wrighton et al., 1996), as well as the respective negative control peptides, were synthesized in the Proteomics Group of the Max Planck Institute of Experimental Medicine and kindly provided by PD Dr. Olaf Jahn. The sequences are listed in Table 5-1. Recombinant human Epo (NeoRecormon, Roche, Welwyn Garden City, United Kingdom) was commercially available and EV-3 was provided by IBA GmbH (Göttingen, Germany) (Bonnas et al., 2017).

Table 5-1: Sequences of Epo-mimetic peptides and control peptides. Control peptides are indicated by "*". HBSP* and P16* contain the same amino acids as HBSP and P16 respectively, but in a different order. EMP1* is a linearized peptide with the same primary sequence as EMP1.

\begin{tabular}{llll}
\hline Peptide & Sequence & Concentration & Sample size \\
\hline \hline HBSP & QEQLERALNSS & $0.11 \mathrm{ng} / \mathrm{ml}$ & 11 \\
\hline HBSP* & QSAQELRESLN & $0.11 \mathrm{ng} / \mathrm{ml}$ & 11 \\
\hline P16 & APPRLICDSRVLERYL & $6.65 \mathrm{ng} / \mathrm{ml}$ & 8 \\
\hline P16* & LYPASPIVLDLRRRCE & $6.65 \mathrm{ng} / \mathrm{ml}$ & 8 \\
\hline EMP1 & $\begin{array}{l}\text { GGTYSCHFGPLTWVCKPQGG } \\
\text { (circularized) }\end{array}$ & $6.65 \mathrm{ng} / \mathrm{ml}$ & 12 \\
\hline EMP1* & $\begin{array}{l}\text { GGTYSCHFGPLTWVCKPQGG } \\
\text { (linearized) }\end{array}$ & $6.65 \mathrm{ng} / \mathrm{ml}$ & 10 \\
\hline
\end{tabular}

\subsubsection{Primary cultures of locust brain cells}

Primary cultures were established from both sexes of fourth-instar nymphs of Locusta migratoria (Hintze, Berlin, Germany). Animals were kept in groups at $24{ }^{\circ} \mathrm{C}$ temperature, $55 \%$ humidity, 12/12 hours light/dark cycle. They were fed with wheat seedings and lettuce leafs ad libitum. Locust brains without optic lobes (typically 1.5 brains per established culture) were dissected and pooled in sterile growth medium consisting of Leibovitz`s L-15 medium (Gibco, Life Technologies, Karlsruhe, Germany) supplemented with $1 \%$ penicillin/streptomycin (Sigma-Aldrich, Munich, Germany) and $1 \%$ amphotericin B (Gibco). Pooling of brains ensures that cells in the same experiment but in different treatment groups are comparable. After rinsing with fresh growth medium, extracted brains were chemically dissociated by $2 \mathrm{mg} / \mathrm{ml}$ collagenase/dispase (Sigma-Aldrich) in growth medium for $30 \mathrm{~min}$ at $27^{\circ} \mathrm{C}$. Following several washing steps with Hanks` Balanced Salt Solution (Gibco), brain tissue was triturated through the tip of a $100 \mu$ pipette to dissociate the cells and to create a homogenous cell suspension. The resulting cell suspension was centrifuged at $3000 \mathrm{~g}$ for 2 min (QuickSpin 700, SüdLaborbedarf, Gauting, Germany) and the cell pellet resuspended in growth medium. Finally, cell suspension was equally allocated onto concanavalin-coated glass coverslips ( $\varnothing 10 \mathrm{~mm}$ ) (Concanavalin A, Sigma-Aldrich) in sterile culture dishes ( $\varnothing 35 \mathrm{~mm}$, Corning Inc., Sigma-Aldrich). After allowing 
attachment of the cells to the coverslip for $2 \mathrm{~h}$, the culture dishes were filled with growth medium that contained $5 \%$ fetal calf serum (FCSG, PAA laboratories, Cölbe, Germany). The cell cultures were maintained at $27^{\circ} \mathrm{C}$ in a humidified normal atmosphere (Incubator, Heraeus, Hanau, Germany). On day 2 and day 4, the medium was replaced by fresh serum-free growth medium. Cell cultures were maintained for five days before experiments started.

\subsubsection{Neuronal survival in hypoxia}

$12 \mathrm{~h}$ prior to hypoxia, cultures of primary locust brain neurons were exposed to serum-free medium without or with Epo-like molecules. The concentrations of Epo-mimetics are summarized in Table 5-1. Epo was applied at a final concentration of $33.6 \mathrm{ng} / \mathrm{ml}$ and EV-3 at a final concentration of $4.2 \mathrm{ng} / \mu \mathrm{l}$ (Miljus et al., 2017). For hypoxia treatment, cells were placed in an airproof chamber (MIC101, Billups-Rothenberg Inc., Del Mar, CA, USA) equipped with an oxygen sensor (Greisinger GOX 100, Conrad Electronics, Hirschau, Germany). Oxygen was replaced by floating the chamber with nitrogen. Hypoxia $\left(\mathrm{O}_{2}\right.$ level $\left.\leq 0.5 \%\right)$ was applied to the cell cultures for $36 \mathrm{~h}$ at room temperature. Control cultures at normoxia were placed next to the hypoxia chamber for the identical time at atmospheric conditions.

\subsubsection{Assessment of neuronal survival}

After hypoxia exposure, cell cultures were maintained for $12 \mathrm{~h}$ in normal atmosphere before fixation with paraformaldehyde (4\%, $30 \mathrm{~min}$ at room temperature). After several washes with $\mathrm{PBS}$ (pH 6.9), the cells were incubated with 4',6-diamidino-2-phenylindole, dihydrochloride (DAPI, Sigma Aldrich) dissolved in PBS at a final concentration of $100 \mu \mathrm{g} / \mathrm{ml}$ for $30 \mathrm{~min}$ in the dark. Finally, excess dye was removed by several washes with PBS before mounting on microscopic slides in 1,4diazobicyclo[2.2.2] octane (DABCO, Roth, Karlsruhe, Germany). Analysis of DAPI fluorescence was performed using an epifluorescence microscope (Zeiss Axioskop; 40x objective) equipped with a Spot

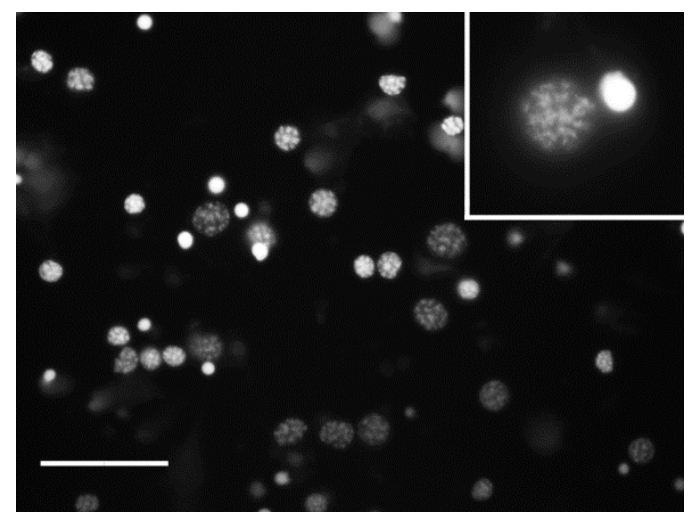

Figure 5-2: DAPI nuclear staining for live/dead cell evaluation. Nuclei of intact cells have larger diameters and display patchy fluorescent patterns that reflect the chromatin structure of the DNA. Nuclei of dead or dying cells are condensed and display intense uniform fluorescence. Inset in upper right corner shows the nuclei of one live (left) and one dead (right) cell. Scale bar: $40 \mu \mathrm{m}$. 
CCD camera (Invisitron, Puchheim, Germany). From each culture, two continuous rows of nonoverlapping photographs (usually around 80-100 per culture) were taken to the right and the left of the center extending over the entire coverslip. Analysis of cell survival (at the time of fixation) was based on DAPI labeled chromatin structure (Figure 5-2) as previously reported for locusts and other insect species (Chao and Nagoshi, 1999; Gocht et al., 2009; Hahn et al., 2017). Nuclei of live and dead neurons were counted and the portion of living cells was calculated for each culture. The person who counted live and dead cells was blinded with respect to the identity of the cultures. Percentages of surviving cells from all treatment groups were normalized to the normoxic control culture of the same experiment (set to 1 ).

\subsubsection{Statistical analysis}

Data analysis and statistics were performed with $R$ (version 3.6.0) using R Studio (version 1.2.1335) (R_Core_Team, 2019; RStudio_Team, 2018). Statistics were calculated with the pairwise permutation test included within the packages "rcompanion" and "coin" (Hothorn et al., 2006, 2008; Mangiafico, 2019). The false discovery rate was controlled using the Benjamini-Hochberg procedure (Benjamini and Hochberg, 1995). Boxplots depict the median, the upper as well as lower quartile, whiskers representing 1.5 times the interquartile range and outliers. Black dots represent the data of individual experiments. 


\subsubsection{Results}

\subsubsection{Neuroprotective effect of Epo-mimetic peptides}

Each experiment directly compared the survival of locust brain neurons under normoxic control conditions with several hypoxia-exposed cultures that were additionally incubated with Epo-mimetic peptides or control peptides with altered structure. To foster equal conditions between experimental groups, all cultures of one experiment were derived from pooled cells of the same animals. Exposure to hypoxia ( $36 \mathrm{~h}$ ) decreased the survival of cultured locust primary brain cells (Figure 5-3). Epo treatment partially reduced hypoxia-induced cell death leading to significantly more $(p=0.0037)$ surviving cells compared to the hypoxia-only condition. Similar to Epo, all three Epo-mimetic peptides significantly increased survival in hypoxia exposed cell cultures (HBSP: $p=0.0040$, EMP1: $p=0.0062) . P 16$ showed the strongest neuroprotective effect $(p=0.0021)$ that fully compensated hypoxia-induced cell loss. In contrast, none of the control peptides (HBSP*, P16* and EMP1*) had a beneficial effect on cell survival in hypoxia-exposed cultures.

A

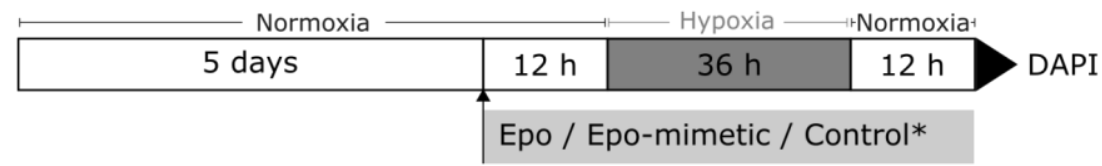

B

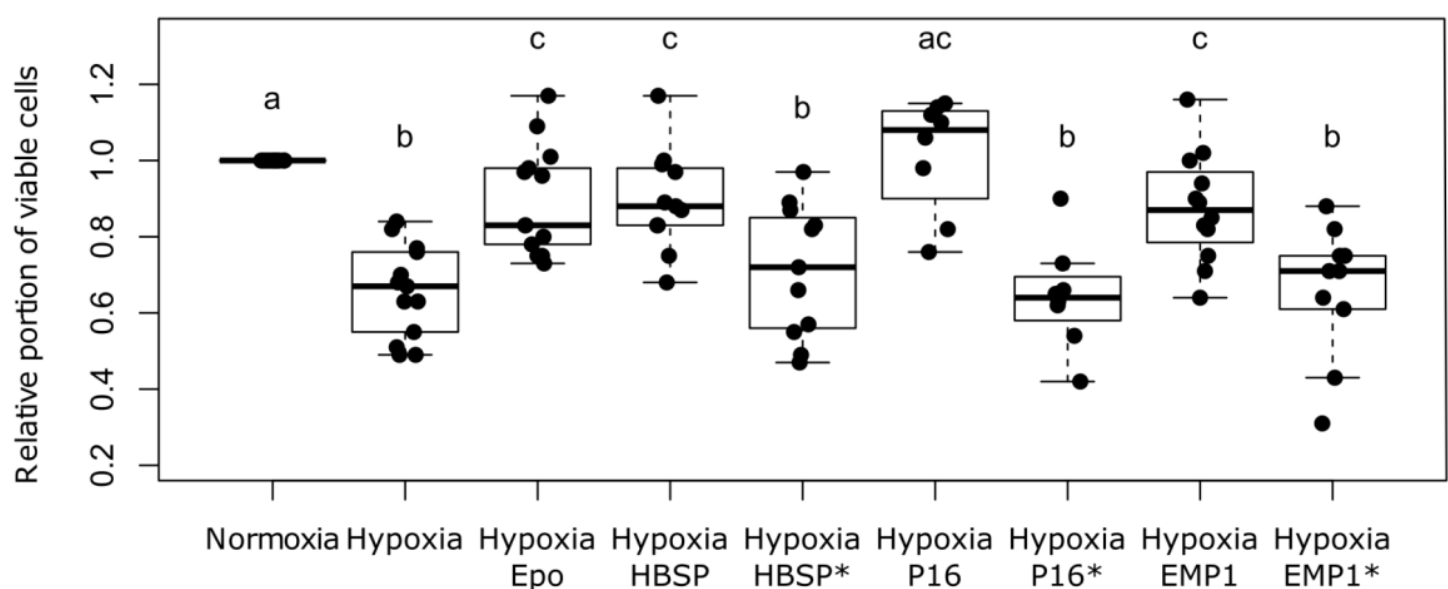

Figure 5-3: Impact of Epo and Epo-mimetic peptides on locust neuronal survival in hypoxia. (A) Experimental procedure. (B) Hypoxia reduces cellular survival compared to normoxic control in vitro. Hypoxia-induced cell death is significantly reduced by Epo, HBSP, P16 and EMP1. Only P16-treated cultures reach normoxic survival rates. "Inactive" control peptides HBSP*, P16* and EMP1* (same concentrations as respective active peptides) elicit no protective effects. Boxplots are overlaid with data of individual experiments (black dots). Overall, 139,988 cells were evaluated. Statistics: Permutation test with BenjaminiHochberg correction. Groups that do not share a letter $(a, b, c)$ are significantly different with at least $\mathrm{p}<0.05$. 


\subsubsection{Neuroprotective effect of EV-3 and P16 after Lm-CRLF3 knock-down}

Locust primary neurons were soaked for 5 days in $10 \mathrm{ng} / \mu \mathrm{l}$ dsRNA to knock-down $L m$-CRLF3 prior to EV-3 or P16 treatment and hypoxia exposure. Cell survival was reduced by $36 \mathrm{~h}$ hypoxia (mean $=0.84)$ compared to normoxic controls (Figure 5-4). This hypoxia-induced cell death was prevented by pre-treatment with EV-3 (mean $=1.06)$ and P16 (mean $=0.99)$. However, their neuroprotective effects were abolished when Lm-CRLF3 was knocked down (EV-3 mean $=0.82$, P16 mean $\mathbf{0 . 7 1}$ ). These preliminary results rely on three biological replicates comprising 24,341 evaluated cells and are currently completed to enable statistical analysis.

A

\begin{tabular}{|l|c|c|c|}
\hline \multicolumn{1}{|c|}{ Normoxia } & $12 \mathrm{~h}$ & $36 \mathrm{~h}$ & $12 \mathrm{~h}$ \\
\hline dsRNA $10 \mathrm{ng} / \mu \mathrm{l}$ & & \\
\hline & $\mathrm{EV}-3 / \mathrm{P} 16$
\end{tabular} DAPI

B

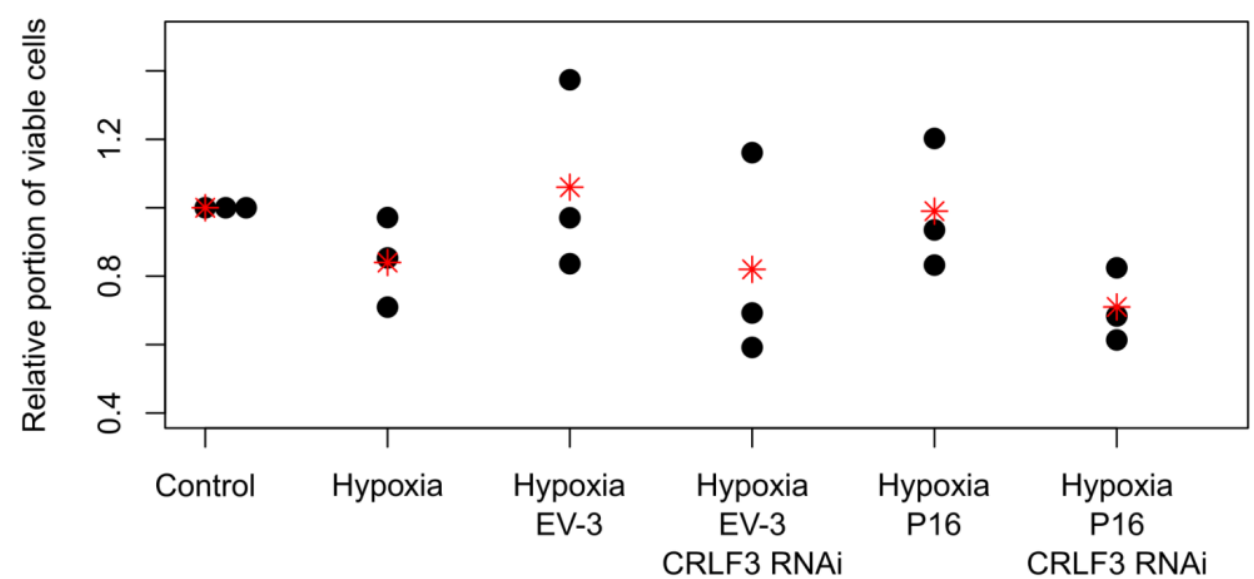

Figure 5-4: Effects of EV-3 and P16 on neuronal survival after Lm-CRLF3 knock-down. (A) Experimental procedure. (B) Hypoxia reduces cellular survival. Pre-treatment with EV-3 and P16 reduces hypoxia-induced cell death. However, this effect is abolished when Lm-CRLF3 is knocked down. Black dots represent data of individual experiments. Means are indicated by a red asterisks. $N=3,24,341$ evaluated cells. 


\subsubsection{Discussion}

To the present, various Epo derivatives have been investigated for neuroprotective and erythropoietic effects in order to find improved drugs to treat neurodegenerative and other diseases (summarized in Table 5-2). Main modification targets have been lysine residues (e.g. carbamylation), charge (e.g. reducing sialylation) or protein/peptide half-life (desialylation). Both lysine residues and

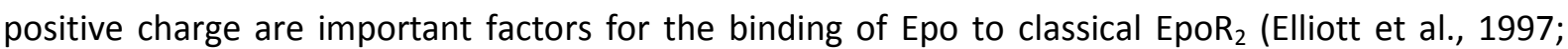
Syed et al., 1998). Furthermore, Epo-like molecules were synthesized that correspond to particular helices of Epo. Most of them are selectively neuroprotective but not erythropoietic (Table 5-2). This selective activation of receptors partly relies on a lower binding affinity to $\mathrm{EpoR}_{2}$. It has been shown that Epo exhibits a lower affinity to tissue-protective Epo-receptors than to EpoR $\mathrm{R}_{2}$ (Brines and Cerami, 2005). Interesting, rather brief ligand availability is sufficient to induce neuroprotection (Brines, 2010; Brines and Cerami, 2005; Morishita et al., 1996). In contrast, a prolonged Epo/EpoR 2 interaction is necessary for stimulation of erythropoiesis (Erbayraktar et al., 2003; Morishita et al., 1996). Although many neuroprotective and other cell protective effects of Epo-like molecules have been demonstrated, their signaling remains rather elusive. AsialoEpo still binds $\mathrm{EpoR}_{2}$ but its half-life is very short and by this is insufficient to induce erythropoietic effects in vivo. Nevertheless, it is sufficient to activate the neuroprotective Epo receptor (Erbayraktar et al., 2003). For cEpo, binding to $E p o R_{2}$ has been excluded. cEpo and HBSP have been shown to activate $E p \circ R / \beta c R$ in vertebrates and are regarded as selective agonists for this heteroreceptor (Chamorro et al., 2013; Leist et al., 2004; Yang et al., 2013). None of the above mentioned Epo-like molecules have been tested in clinical trials since pre-clinical studies still struggle with too short half-lives and appropriate peptide administration. Peptides like HBSP, P16 and EMP1 are advantageous due to their small size which may increase absorption and crossing of the blood brain barrier making them promising candidates for clinical use (Arranz-Gibert et al., 2019). P16 (16 amino acids) and HBSP (11 amino acids) are the shortest peptides tested so far (compare Table 5-2).

Our results show that the synthesized and slightly modified peptides P16, HBSP and EMP1 exert a robust neuroprotective effect on locust primary brain cells in vitro. P16, representing a part of Epo's helix $A$, elicits even higher protective properties compared to Epo (Figure 5-3). In order to exclude non-specific effects, we treated cells from the same pool of brains with control peptides P16*, HBSP* and EMP1*. P16* and HBSP* were scrambled but consisted of identical amino acids as their counterparts. EMP1* was linearized whereas EMP1 is a circularized peptide. In contrast to the other peptides, EMP1 is neuroprotective and erythropoietic in vertebrates which might be due to its circularized form (Kawakami et al., 2001; Livnah et al., 1996; Wrighton et al., 1996). 
Table 5-2: Summary of previously investigated Epo-like molecules and their functions.

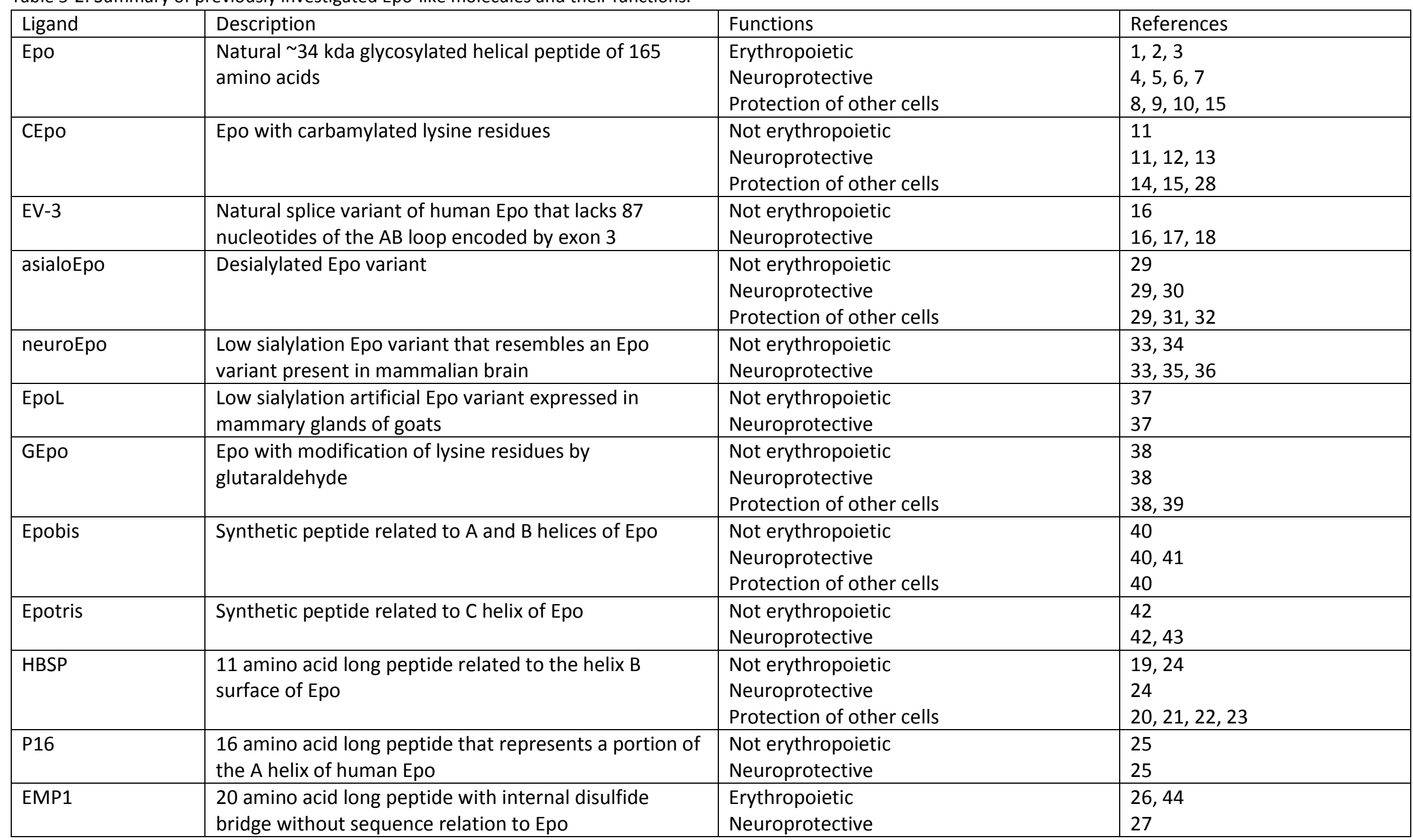


1 (Erslev, 1953); 2 (Jelkmann, 1992); 3 (Constantinescu et al., 1999); 4 (Sirén et al., 2001); 5 (Dame et al., 2001); 6 (Morishita et al., 1996); 7 (Ostrowski et al., 2011); 8 (Zou et al., 2016); 9 (Calvillo et al., 2003); 10 (Szenajch et al., 2010); 11 (Leist et al., 2004); 12 (Chamorro et al., 2013 ); 13 (Ding et al., 2017 ); 14 (Tögel et al., 2016); 15 (Erbayraktar et al., 2009); 16 (Bonnas et al., 2017); 17 (Miljus et al., 2017); 18 (Hahn et al., 2017); 19 (Ahmet et al., 2011 ); 20 (Yang et al., 2014 ); 21 (Ahmet et al., 2013); 22 (Zhang et al., 2017); 23 (Yang et al., 2013); 24 (Brines et al., 2008); 25 (Bonnas, 2009 ); 26 (Livnah et al., 1996$) ; 27$ (Kawakami et al., 2001);28 (Moon et al., 2006);29 (Erbayraktar et al., 2003);30 (Wang et al., 2004);31 (Kittur et al., 2019);32 (Mennini et al., 2006);33 (Rodríguez Cruz et al., 2010);34 (Lagarto et al., 2011);35 (Fernando et al., 2018);36 (Pedroso et al., 2018);37 (Castillo et al., 2019);38 (Chattong et al., 2013 );39 (Sooklert et al., 2016);40 (Dmytriyeva et al., 2016);41 (Pankratova et al., 2012);42 (Pankratova et al., 2010);43 (Zellinger et al., 2011);44 (Wrighton et al., 1996). 
Circularization of peptides commonly increases their half-life which has also been demonstrated for HBSP and a circularized variant termed cyclized helix B peptide (CHBP) (Yang et al., 2014). CHBP is stable in human plasma for more than $300 \mathrm{~min}$ in contrast to linear HBSP (half-life 9-10 min) in vitro. In a hepatocyte model system, the half-life of CHBP was 2.5 times longer than that of HBSP. Furthermore, the in vivo half-life of CHBP is remarkably prolonged in comparison to HBSP in rats (Yang et al., 2014). As mentioned before, longer half-life of Epo-like molecules increase the chance of activating $\mathrm{EpoR}_{2}$. Unfortunately, it has not been tested whether CHBSP is only neuroprotective or also erythropoietic. None of the control peptides in this study affected cell viability underlining that the neuroprotective effect of their counterparts was specific. It was particularly important to test circularized EMP1 and linearized EMP1* in our in vitro assay. In vivo EMP1* could still be neuroprotective but not erythropoietic due to its expected shorter half-life. Our in vitro data demonstrates that the neuroprotective effect of EMP1 is rather independent of the half-life. In contrast, the neuroprotective effect is likely based on peptide conformation since the linearized EMP1* was not neuroprotective. Whether the linearize form is still erythropoietic cannot be assessed in locust cells due to the lack of $\mathrm{EpoR}_{2}$ and should be tested in appropriate vertebrate cells (e.g. UT-7). Our study provides evidence that all tested peptides share structural similarities inducing neuroprotection. This knowledge offers the possibility to find the motif necessary for stimulation of neuroprotection. Primary sequence alone is not conclusive since EMP1 is unrelated to Epo and HBSP is composed of non-consecutive amino acids of Epo's helix B. It is likely, that the peptides share a 3D conformation building a common binding motif. The overall structure might be further adjusted by particularly orientated amino acids or the distribution of charged amino acids. To this end, bioinformatic approaches that predict molecular structures will help to find their commonalities. In addition, HBSP, which consists of only 11 amino acids, could be further shortened to determine the smallest number of required amino acids.

Given that the long-term objective is to develop neuroprotective therapies, it is pivotal to characterize the signaling pathway mediating neuroprotective effects in order to assess potential adverse side effects. We have chosen P16 and EV-3 for further analysis since P16 and EV-3 are the most promising for clinical use. P16 showed the most protective effect in locusts being even more protective than Epo. It cannot be excluded, that further optimizing the concentration of the other Epo-mimetic peptides might enhance their neuroprotective effect as well. EV-3 is a human splice variant that selectively induces neuroprotection and is endogenously present in fetal brain but not in adult kidney (Bonnas et al., 2017). The robust neuroprotective effect of EV-3 on locust primary brain cells has previously been demonstrated (Miljus et al., 2017). EMP1 is not only neuroprotective but also erythropoietic thereby reducing its suitability for clinical use. Hence, we tested whether P16 and EV-3 induce neuroprotection of locust neurons via activation of Lm-CRLF3. Orthologs of other 
neuroprotective Epo receptors including EpoR, EphB4 and the heterodimeric EpoR/ $\beta c R$ receptor are generally missing in insects. Thus, contribution of these receptors can be excluded in insect cells in contrast to vertebrate cells. Previous studies with Epo on locust and beetle neurons suggested that the neuroprotective effect was exclusively mediated by CRLF3, with no additional receptor being involved (Hahn et al., 2017, 2019). We knocked down Lm-CRLF3 via soaking RNAi and found that the neuroprotective effect of EV-3 and P16 was abolished (Figure 5-4). As a consequence, Lm-CRLF3 is crucial for EV-3 and P16 induced neuroprotection as it is also for Epo-mediated neuroprotection.

Future studies should examine whether EV-3 and P16 directly bind to Lm-CRLF3 or indirectly induces receptor activation. It is likely that CRLF3 is a membrane bound receptor forming homodimers or heterodimers similar to the other class 1 cytokine receptors members (Liongue and Ward, 2007). To the present, we have demonstrated that CRLF3 is a crucial component of Epo-induced neuroprotection in insects. However, no binding assays with Epo or Epo-mimetic peptides have been performed yet. CLRF3 might be present at the cell surface as a homodimer directly binding Epo-like molecules to activate STAT signaling pathways. However, CRLF3 might also form heterodimers with an unknown receptor which binds to Epo and then indirectly activates CRLF3. Furthermore, it cannot be excluded that CRLF3 lacks a transmembrane domain and forms a heterodimer with another transmembrane receptor to propagate activation to intracellular signaling pathways (Liongue et al., 2016).

These results show that $L m$-CRLF3 is not only involved in Epo-induced neuroprotection but is also activated by neuroprotective but non-erythropoietic Epo-like variants in insects (Hahn et al., 2019). Since human recombinant Epo, the human endogenous splice EV-3 and P16 induce neuroprotection in locust primary brain cells via Lm-CRLF3, it is highly probable that human Hs-CRLF3 mediates neuroprotective functions of Epo-like molecules as well. This is further supported by the extraordinary high conservation of CRLF3 proteins in Eumetazoa and its emergence together with the origin of the eumetazoan nervous system (Hahn et al., 2019). 


\subsection{Tc-crlf3 expression in Tribolium castaneum}

This subchapter focuses on the analysis of Tc-crlf3 expression in various developmental stages of Tribolium castaneum by quantitative real-time PCR (qRT-PCR) and in situ hybridization (ISH). It represents the basis for a future manuscript that will be complemented by data investigating different Tribolium tissues and TC-CRLF3 protein abundance. These studies were conducted by several people that will be listed as potential authors below. I contributed to the design of the study and supervised the bachelor student Henning Jelten who substantially supported me in data collection. Furthermore, I designed, cloned and prepared the probes for ISH staining, performed the larval brain ISH stainings and analyzed as well as visualized the data. Finally, I wrote the manuscript.

Authors will be Nina Hahn, Henning Jelten, Felix Kaufholz, Vera S. Hunnekuhl, Marita Büscher, Gregor Bucher and Ralf Heinrich. Potential authors will be Jonas G. Reh and Jannis Hainke whose Bachelor theses presumably will be related to that topic.

$\mathrm{NH}$ and $\mathrm{HJ}$ collected data. NH, FK and VSH supervised the study and were involved in troubleshooting. $\mathrm{NH}, \mathrm{FK}, \mathrm{VSH}, \mathrm{MB}, \mathrm{GB}$ and $\mathrm{RH}$ designed the study. $\mathrm{NH}$ and $\mathrm{HJ}$ analyzed data. $\mathrm{NH}$ visualized data and wrote the manuscript.

We thank Daniel Jackson for advice regarding in situ probe design and Georg Oberhofer for providing reference primers. 


\subsubsection{Introduction}

The cytokine receptor-like factor 3 (CRLF3) is termed an orphan cytokine receptor because its endogenous ligand remains to be identified. It is well conserved throughout the animal kingdom and emerged with the origin of the eumetazoan nervous system (Hahn et al., 2019). CRLF3 is a member of the class I group 1 cytokine receptors which also includes the classical erythropoietin receptor $(E p o R)$, the thrombopoetin receptor, the prolactin receptor and the growth hormone receptor. All receptors are transmembrane receptors characterized by the extracellular WSXWS motif and a fibronectin type III (FN III) domain. Group 1 receptors typically form homodimers to bind four-helix bundle cytokines (Boulay et al., 2003; Liongue and Ward, 2007). If CRLF3 is activated by its unknown endogenous ligand, it induces signal transducers and activators of transcription 3 (STAT3). CRLF3 induces G0/G1 cell cycle arrest and prevents entry into the $S$ phase (Yang et al., 2009). Its overexpression leads to an upregulation of cyclin D1 and D3, both being involved into cell cycle progression (Yang et al., 2009; Zhang et al., 2014a). CRLF3 is expressed in various human tissues such as brain, liver, spleen or pancreas. Moreover, CRLF3 expression has been demonstrated to be upregulated in tumors (Yang et al., 2009). Besides cell cycle regulation, CRLF3 is required for neuronal morphology and is associated with amyotrophic lateral sclerosis (Cirulli et al., 2015; Hashimoto et al., 2012).

In previous studies, we have demonstrated that insect orthologs of CRLF3 are crucial for erythropoietin-induced neuroprotection in primary brain cells of the beetle Tribolium castaneum and the locust Locusta migratoria (Hahn et al., 2017, 2019). These cells are sensitive to human erythropoietin (Epo) which induces an anti-apoptotic effect particularly when cells are challenged by hypoxia. However, if CRLF3 expression is down regulated, Epo-mediated neuroprotection is abolished, indicating that CRLF3 is critically involved in the neuroprotective signaling pathway. The fact that human Epo induces neuroprotection in insects, even though no endogenous Epo or EpoR is expressed, suggests that an Epo-like signaling system might have been present in a common urbilaterian ancestor (Hahn et al., 2019; Ostrowski et al., 2011). Given that CRLF3 is well conserved and that human Epo protects Tribolium neurons from apoptosis, the beetle is a suitable model system to further investigate the orphan cytokine receptor CRLF3. It is likely that insights into Tribolium CRLF3 signaling and regulation are transferable to vertebrates.

Investigating CRLF3 provides new insight into Epo-mediated neuroprotection. In vertebrates, Epo stimulates both cell protection and erythropoiesis. This dual function of Epo leads to adverse side effects that are related to its erythropoietic function when applied as a neuroprotective drug. A phase II/III clinical trial using Epo to treat acute ischemic stroke revealed that Epo administration increased the risk of thromboembolism (Ehrenreich et al., 2009). Thus, safe alternative drugs need to 
be developed that, specifically activate neuroprotective receptors but not the classical EpoR, which stimulates erythropoiesis. EV-3 is a human splice variant that selectively stimulates neuroprotection but not erythropoiesis thereby indicating that neuroprotection and erythropoiesis are mediated by different receptors (Bonnas et al., 2017). Several alternative Epo receptors including the $\beta$-common and Ephrin B4 receptor have been discussed, but Epo-mediated neuroprotection remains partially elusive (Brines et al., 2004; Pradeep et al., 2015; Um et al., 2007). Hence, we aim to characterize the neuroprotective Tribolium Epo receptor Tc-CRLF3, whose human ortholog is assumed to be a potential neuroprotective Epo receptor as well.

Our previous experiments show that beetle $T c-c r l f 3$ must be expressed in brain neurons of Tribolium pupae (Hahn et al., 2017). However, nothing is known about its expression pattern in the brain and its regulation upon hypoxia exposure. Hence, we qualitatively investigated Tc-crlf3 expression in the embryo and larval brain by ISH staining. Furthermore, we quantitatively examined Tc-crlf3 expression in different developmental stages and following hypoxia exposure. Tribolium castaneum is a suitable model organism since its genome is well annotated and, in contrast to Drosophila melanogaster, it expresses an ortholog of CRLF3 (Hahn et al., 2017, 2019; Richards et al., 2008). In addition, there are many genetic tools available and well established including ISH and parental RNAi (pRNAi) (Bucher et al., 2002; Schinko et al., 2009; Shippy et al., 2009). pRNAi induces a knock-down that is transferred from the injected females to the offspring and thus provides an excellent negative control to determine the specificity of RNA probes for ISH (Bucher et al., 2002). 


\subsubsection{Material and methods}

\subsubsection{Animals}

Tribolium castaneum beetles of the wild-type strain San Bernadino were reared in plastic boxes with a mesh area in the lid for ventilation at $32{ }^{\circ} \mathrm{C}$ and $40 \%$ humidity. They were raised on full grain flour supplemented with $5 \%$ dry yeast and transferred to type 405 flour with yeast for egg laying. Eggs, larvae and pupae were separated by differently sized mesh sieves (Brown et al., 2009). $5^{\text {th }}$ instar larvae were identified by their size and age. In addition, beetles of the enhancer trap line G10011 from the GEKU screen were utilized (Trauner et al., 2009). They express a specific egfp pattern in the head and hindgut and were used to distinguish between pRNAi treated and non-treated embryos within the same vial after ISH. Since this study was conducted exclusively with insects, it does not require a special permission. All experiments comply with the German laws for animal welfare ("Deutsches Tierschutzgesetz").

\subsubsection{Oligonucleotides}

Table 5-3: Summary of primers for qRT-PCR analysis and cloning of Tribolium genes.

\begin{tabular}{|c|c|c|}
\hline Primer & Nucleotide sequence & Reference \\
\hline Tc-crlf3_ISH F & 5' GACACGCCTTCGAACCGTCA 3' & This study \\
\hline Tc-crlf3_ISH R & 5' CCAACGGCAAATTGTGGTCCATC 3' & This study \\
\hline qTc-crlf3 F & 5' GCGATCACAGCTATTTTTGCTCTTG 3' & This study \\
\hline qTc-crlf3 R & 5' ACACGTATCAATACTGACTCGTAC 3' & This study \\
\hline Atub 3F1 & 5' CGCCAATAACTACGCCAGAG 3' & Dr. Georg Oberhofer \\
\hline Atub 3R1 & 5' CGAACGAGTGGAAAATCAAGAA 3' & Dr. Georg Oberhofer \\
\hline GAPDH $3 F 1$ & 5' CGTTTCCGTTGTGGATTTGAC 3' & Dr. Georg Oberhofer \\
\hline GAPDH $3 R 1$ & 5’ AACGACCTCTTCCTCCGTGTA 3' & Dr. Georg Oberhofer \\
\hline RPS 3.3F1 & 5' AGGGTGTGCTGGGAATTAAAG 3' & Dr. Georg Oberhofer \\
\hline RPS 3.3R1 & 5’ GGGTAGGCAGGCAAAATCTC 3' & Dr. Georg Oberhofer \\
\hline M13F & 5' GTAAAACGACGGCCAGT 3' & Standard primer \\
\hline M13R-T7 & 5' taatacgactcactataggCAGGAAACAGCTATGAC 3' & Standard primer \\
\hline
\end{tabular}

Primers for reference genes were kindly provided by Dr. Georg Oberhofer. 


\subsubsection{In situ hybridization}

ISH staining of embryos was performed as described previously with slight modifications (Schinko et al., 2009). Embryos were fixed with $2 \mathrm{ml} \mathrm{PEMS} \mathrm{(0.1} \mathrm{M} \mathrm{Pipes,} 2 \mathrm{mM} \mathrm{MgSO}_{4}, 1 \mathrm{mM}$ EDTA, pH 6.9.), $6 \mathrm{ml}$ heptane and $250 \mu \mathrm{l} 37 \%$ formaldehyde for $30 \mathrm{~min}$ on an orbital shaker. To rehydrate the embryos carefully, they were consecutively washed in $75 \%, 50 \%$ and $25 \%$ methanol/PBT prior post-fixation in $4 \%$ formaldehyde/PBT for $15 \mathrm{~min}$. Proteinase $\mathrm{K}$ treatment was omitted. After hybridization, washing steps were performed using MABT $(100 \mathrm{mM}$ maleic acid, $150 \mathrm{mM} \mathrm{NaCl} ; 0.1 \%$ Triton-X, pH 7.4) and MABT supplemented with Roche blocking reagent (\#11096176001) was used for blocking steps $(1 \mathrm{~h})$. The probes were labeled with digoxigenin (DIG) and detected by NBT/BCIP color reaction (\#11681451001, Roche). For the detection of egfp in G10011 embryos the eyfp (enhanced yellow fluorescent protein) probe (kindly provided by the Bucher Lab, Goettingen, Germany) was used. The sequence targeting Tc-crlf3 was cloned into the vector pCRII (TA Cloning ${ }^{\circledR}$ Kit Dual Promoter with pCR ${ }^{\mathrm{TM}}$ II vector, Thermo Fisher Scientific, Germany) as described earlier (Hahn 2017, 2019) with the primers Tc-crlf3_ISH forward and reverse. RNA probes were synthesized using the DIG RNA labeling mix (\#11 277073 910, Roche, Germany) and the Sp6 (\#10 810274 001, Roche, Germany) or T7 RNA polymerase (\#10 881767 001, Roche, Germany), respectively. Staining of embryos was analyzed using a ZEISS Imager Z2 microscope and Zen software (Blue Edition) (Carl Zeiss Microscopy, Jena, Germany)

Whole mount larval brains were dissected and stained as described in (Hunnekuhl et al., 2020) using DIG-labelling and the tyramide signal amplification (TSA). The staining was analyzed with the aid of a Leica SP8 confocal microscope (Wetzlar, Germany), and the Las X software (version 3.5.2.18963). Images were processed (brightness, contrast, rotation, color composite) in Fiji and combined into a figure using the ScientiFig plug-in (Aigouy and Mirouse, 2013; Schindelin et al., 2012).

\subsubsection{Parental RNAi}

Parental RNAi was performed as described elsewhere injecting 350 pupae (Bucher et al., 2002). dsRNA that targets the Tc-crlf3 fragment 1 was synthesized as stated in (Hahn et al., 2017). Controls

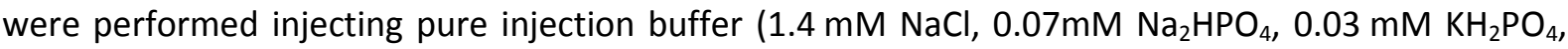
$4 \mathrm{mM} \mathrm{KCl}$ ). The efficacy of knock-down in the offspring was verified by qRT-PCR. In addition, the offspring were examined for variations in cuticle morphology and survival. Specimens were cleared using Hoyer's mounting medium and analyzed with a ZEISS Axioplan 2 Microscope (Wohlfrom et al., 2006). Furthermore, the number of empty egg shells was counted. These eggs died prior to cuticle development and an elevated number of empty egg shells would indicate severe developmental defects. 


\subsubsection{RNA extraction, cDNA synthesis and quantitative real-time PCR}

For each RNA extraction, 5 larvae ( $5^{\text {th }}$ instar), pupae or adults were first washed in water. Embryos (50 each) were dechorionized prior to RNA extraction by alternating washing steps in water and $50 \%$ bleach (diluted in water) starting and ending in water. Brains for RNA extraction were freshly dissected in sterile PBS and collected in $800 \mu$ l lysis buffer. RNA was extracted using the ZR Tissue \& Insect RNA MicroPrep ${ }^{\mathrm{TM}}$ Kit (\#R2030, Zymo Research). Tissues were homogenized using a pestle, centrifuged $(12,000 \mathrm{~g}, 1 \mathrm{~min}, \mathrm{RT})$ and the supernatant was loaded onto the Zymo-Spin IIIC Column. The following steps were performed according to the user manual including the on-column DNAse I treatment.

Reverse transcription of $1 \mu \mathrm{g}$ total RNA into cDNA was performed using the QuantiTect Reverse Transcription Kit (\#205311, Qiagen, Germany). For qRT-PCR, 10 ng cDNA were combined with $5 \mu$ of iTaq $^{\text {TM }}$ Universal SYBR ${ }^{\circledR}$ Green Supermix (\#1725121, Bio-Rad, Germany) and $0.1 \mu \mathrm{M}$ primers in a final reaction volume of $10 \mu \mathrm{l}$ in 96-well plates (\#HSS9665, Bio-Rad, Germany) covered with a seal (\#MSB1001, Bio-Rad, Germany). The reference genes were Tc-gapdh, Tc-rps3.3 and Tc-atub. Primers were tested for efficiency and stability and three technical replicates were included.

Amplification was conducted by a $\mathrm{MyiQ}^{\mathrm{TM}}$ Single-ColorReal-Time PCR Detection System (\#170-9740, Bio-Rad, Germany) applying the following program: $95^{\circ} \mathrm{C}$ for $3 \mathrm{~min}$ followed by 40 cycles of $95^{\circ} \mathrm{C}$ for $10 \mathrm{~s}, 60^{\circ} \mathrm{C}$ for $30 \mathrm{~s}$ and $72{ }^{\circ} \mathrm{C}$ for $30 \mathrm{~s}$, and a final step at $95^{\circ} \mathrm{C}$ for $1 \mathrm{~min}$. A melting curve analysis was performed starting at $55^{\circ} \mathrm{C}$ for $1 \mathrm{~min}$ and increasing the temperature in $81 \mathrm{cycles}$ for $0.5^{\circ} \mathrm{C}$ every $10 \mathrm{~s}$ up to $95^{\circ} \mathrm{C}$. Data was analyzed by the comparative $C_{T}$ method (Livak and Schmittgen, 2001). $C_{T}$ values of reference genes were combined calculating the geometric mean.

\subsubsection{Hypoxia treatment}

Animals were exposed to hypoxia $\left(\mathrm{O}_{2}\right.$ level $\left.<0.2 \%\right)$ for $36 \mathrm{~h}$ in a hypoxic chamber $(\# 27310$, STEMCELL ${ }^{\text {TM }}$, Germany) flooded with nitrogen. Controls were simultaneously exposed to normoxia next to the hypoxia chamber at ambient room temperature conditions. 


\subsubsection{Results}

\subsubsection{Parental RNAi of $T c$-crlf3}

The efficiency of pRNAi was measured in $0-24 \mathrm{~h}$ old embryos 7 days and 28 days after injection of the mother (Figure 5-5). After 7 days, Tc-crlf3 expression in embryos was reduced to $6 \%$ compared to mock injected mothers. Hence, these embryos displayed a robust knock-down and are well suited as negative controls for in situ stainings in order to judge the staining specificity. However, embryonic Tc-crlf3 expression increased 1.64-fold in comparison to offspring of mock injected animals after 28 days.

In order to assess whether knock-down of CRLF3 induced lethal, developmental or morphological effects, cuticles as well as viability of embryos and larvae were evaluated. The empty egg shell ratio of pRNAi females did not differ from the controls ( $27 \%$ vs. $32 \%$ ). Furthermore, the cuticles of 25 pRNAi larvae and 15 control larvae were analyzed. No abnormalities were observed.

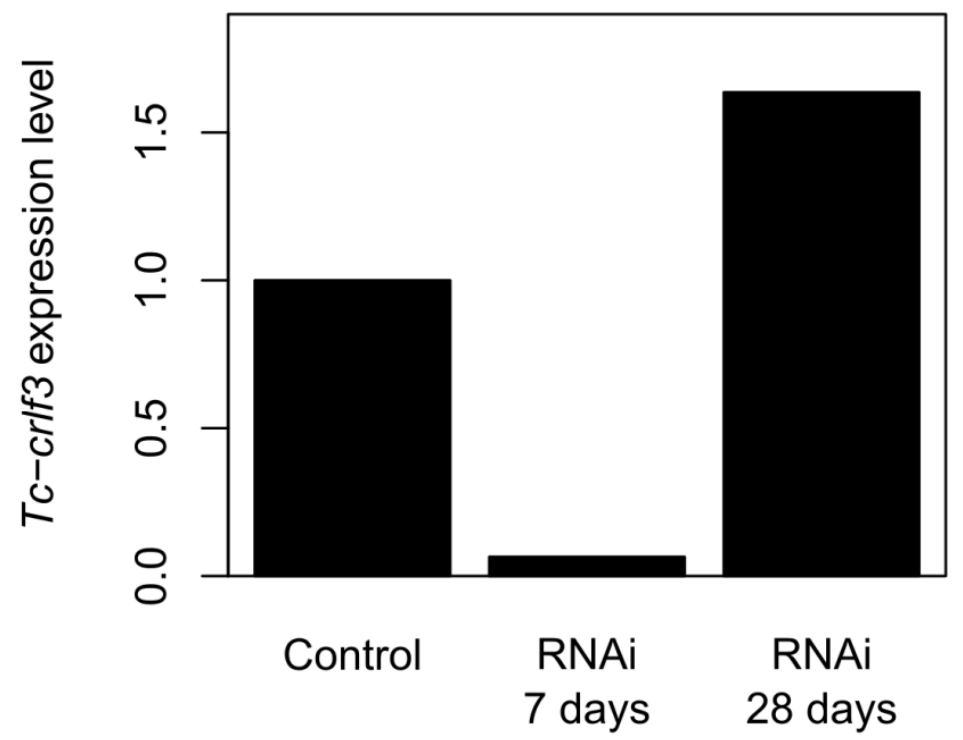

Figure 5-5: Evaluation of Tc-crlf3 expression after parental RNAi injecting pupae. Embryos after 7 days are affected by a $94 \%$ knock-down whereas embryos after 28 days express $64 \%$ more $T c$-crlf3. $\mathrm{N}=1$.

\subsubsection{In situ hybridization stainings in embryos and the larval brain}

Day 8 pRNAi embryos and G10011 embryos were fixed and stained together in the identical vial. pRNAi embryos suffered from a Tc-crlf3 knock-down whilst and G10011 embryos showed wild-type $T c$-crlf3 expression. Samples could be differentiated by additional egfp expression in G10011 animals. egfp expression was detected by eyfp probes. Figure 5-6 shows NBT/BCIP color reaction stainings of 24 - $48 \mathrm{~h}$ embryos. In addition, Supplementary Figure 5-1 shows close ups of the head region. Images $A$ and B in both figures depict the staining pattern of G10011 and pRNAi embryos stained with a 
mixture of eyfp and Tc-crlf3 probes. Images C-E show the stainings with single probes, respectively. The staining elicited by the eyfp probe prominently marks regions in the head and hindgut $(A+C)$ and is only present in G10011 embryos but not in pRNAi embryos (B). Tc-crlf3 staining is weaker and evenly located throughout the body $(A+D)$. However, this staining is not present in pRNAi embryos treated in the identical way $(B+E)$.

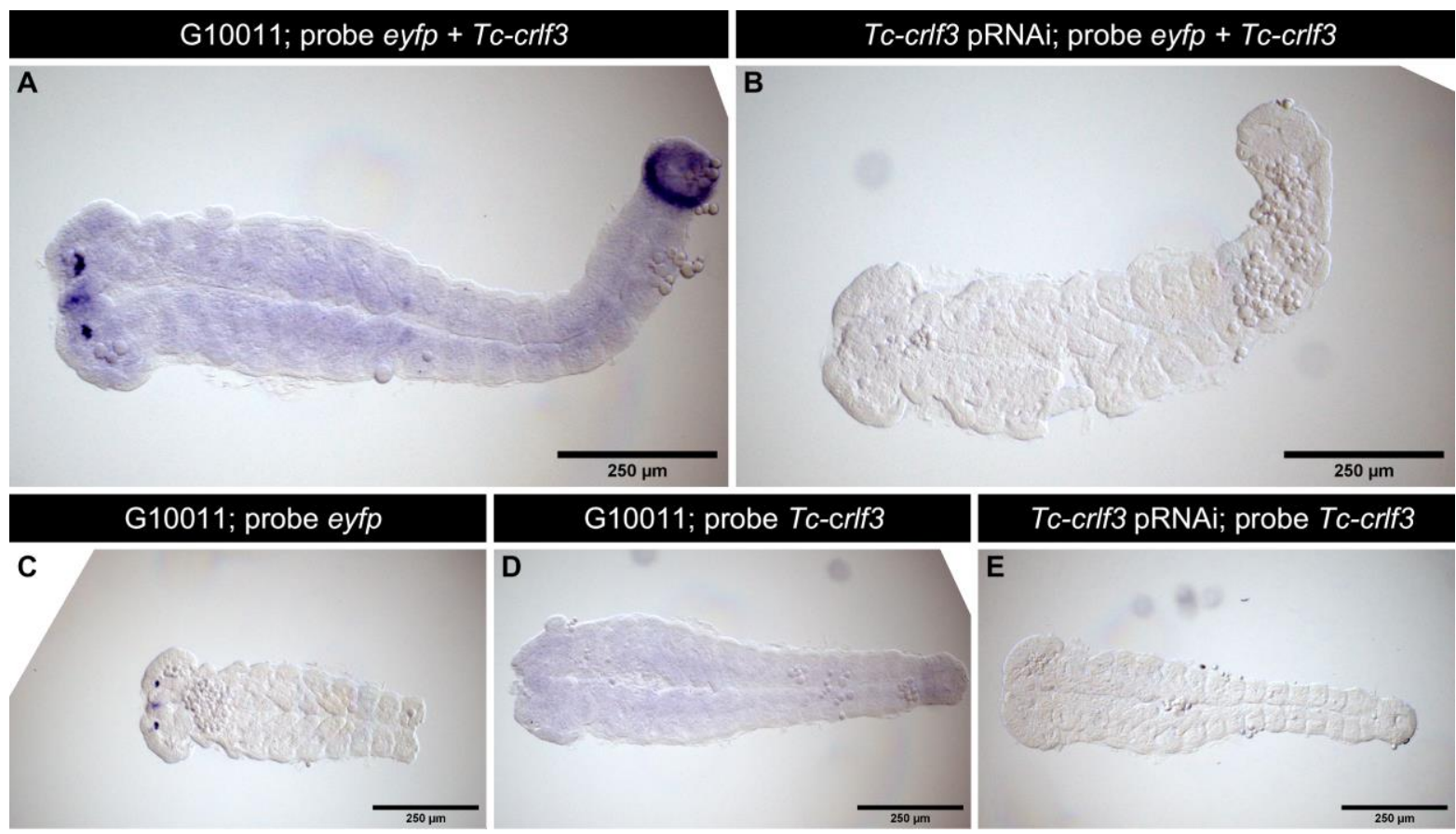

Figure 5-6: 24 - $48 \mathrm{~h}$ old whole-body embryos stained by a NBT/BCIP color reaction of the Tc-crlf3 and eyfp (detecting egfp) probes for $19 \mathrm{~h}$ at $4^{\circ} \mathrm{C}$. The white balance of each image is identical. (A) G10011 embryo stained with a mixture of both Tc-crlf3 and eyfp probes, (B) pRNAi embryo stained with a mixture of both Tccrlf3 and eyfp probes, (C) G10011 embryo stained with the eyfp probe, (D) G10011 embryo stained with the $T c$-crlf3 probe, (E) pRNAi embryo stained with the Tc-crlf3 probe.

As the specificity of Tc-crlf3 probe has been proven in Tribolium embryos, it was used to stain larval brains ( $5^{\text {th }}$ instar). The larval Tc-crlf3 staining with $\alpha$-Synpasin and DAPI counterstaining is shown in Figure 5-7 (A -D overview, $A^{\prime}-D^{\prime}$ close up). Tc-crlf3 staining (red) neither overlaps with DAPI (blue) nor with $\alpha$-Synpasin (green) staining. The staining is strong in the outer rind of the larval brain with weak staining between neuropil compartments. 

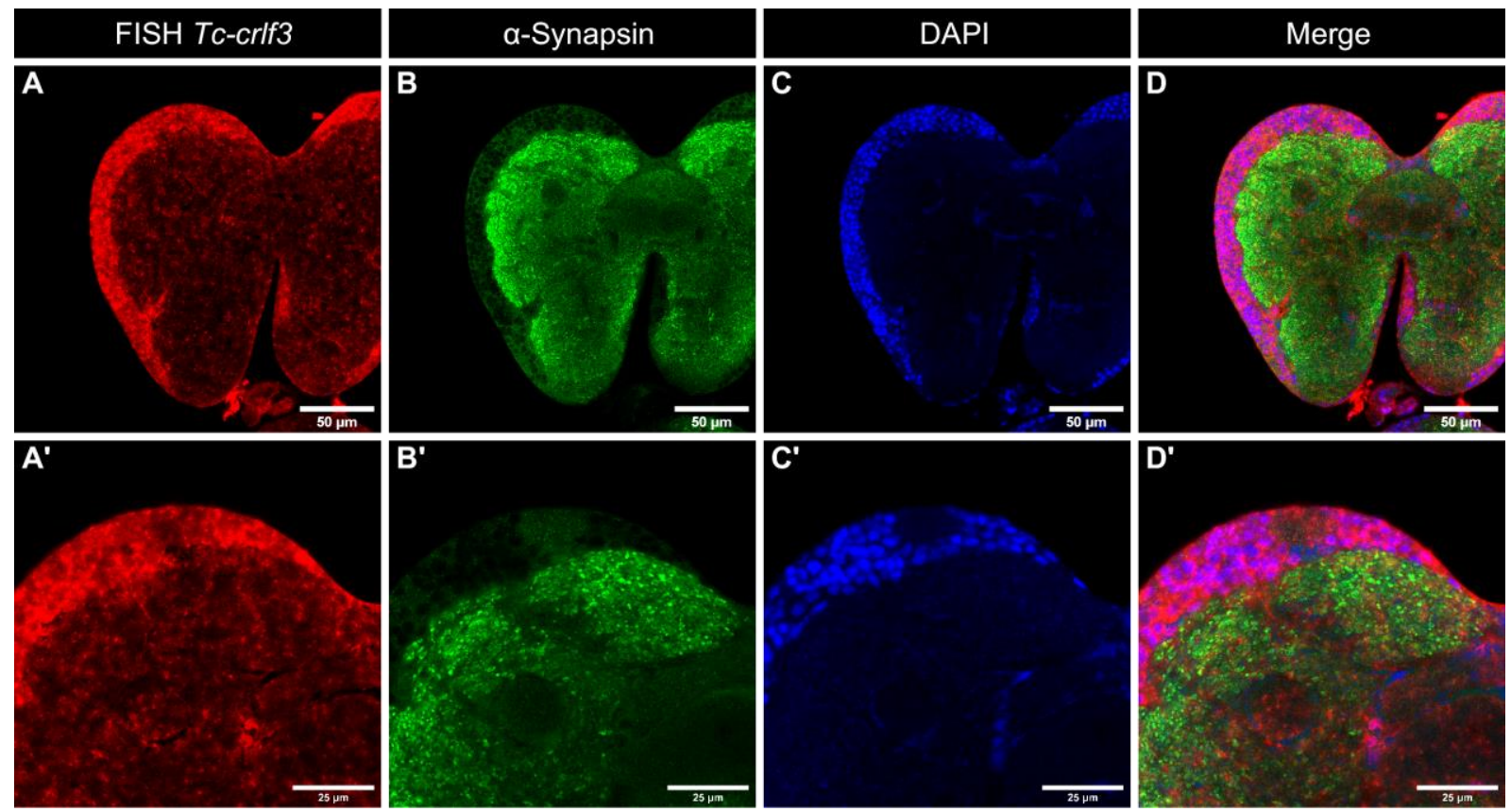

Figure 5-7: Fluorescent in situ staining (FISH) in Tribolium whole mount larval brains. The bottom row shows close ups from upper row images. (A, $\left.\mathbf{A}^{\prime}\right)$ Tc-crlf3 (red) is predominantly expressed in the outer rind, (B, $\left.\mathbf{B}^{\prime}\right)$ Synapsin (green) counterstaining visualizes the anatomy of the brain by particularly staining neuropils, (C, $\mathbf{C}^{\prime}$ ) DAPI (blue) labels nuclei, (D,D') Merge of the red, green and blue channels.

\subsubsection{Stage-dependent Tc-crlf3 expression}

In order to determine whether Tc-crlf3 expression changes during development, it was investigated in embryos (0 - 24 h, $24-48$ h, $48-72$ h), larvae, pupae and adults (Figure 5-8). The expression was normalized to the expression of $T c-c r l f 3$ in adults. In embryos and pupae, $T c-c r l f 3$ expression is higher

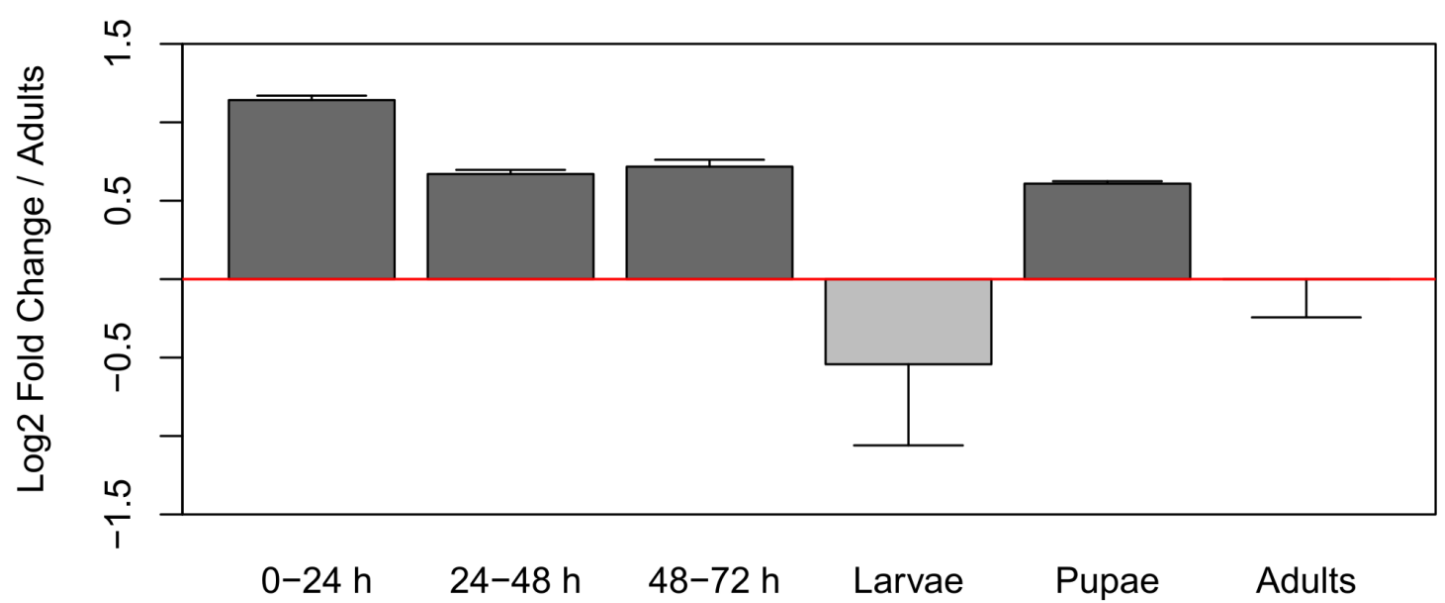

Developmental stage

Figure 5-8: Expression levels of Tc-crlf3 in different developmental stages. Expression normalized to the level in adults (set to 0 ). Dark grey bars represent higher expression than in adults, light grey bar represents lower expression than in adults. Bars show the mean and standard deviation. $\mathrm{N}=3$. 
than in adults being the highest in $0-24 \mathrm{~h}$ old embryos $\left(2^{\wedge} 1.14 \pm 0.03\right.$ fold $)$. In contrast, the expression in larvae is lower than in adults $\left(2^{\wedge}(-0.54) \pm 0.52\right.$ fold $)$. Furthermore, the expression level in larvae is more variable than at other developmental stages.

\subsubsection{Effect of $36 \mathrm{~h}$ hypoxia on $\mathrm{Tc}$-crlf3 expression}

Moreover, the effect of hypoxia on Tc-crlf3 expression was investigated at different developmental stages (Figure 5-9). In the youngest embryos (18 - $24 \mathrm{~h}$ ), hypoxia increased Tc-crlf3 expression $\left(2^{\wedge} 2.97 \pm 0.21\right.$ fold $)$ compared to control embryos reared at normoxia. In adults, hypoxia might upregulate Tc-crlf3 expression; however, variations are rather large including samples without considerable impact on expression level. In older embryos (48 - $72 \mathrm{~h}$ ), larvae and pupae, Tc-crlf3 expression seemed to decrease $\left(2^{\wedge}(-0.45) \pm 0.35\right.$ fold, $2^{\wedge}(-0.29) \pm 0.42$ fold, $2^{\wedge}(-0.41) \pm 0.27$ fold, respectively). Though, variations are noteworthy as well. In general, variations after hypoxia exposure are remarkably higher in each developmental stage (Figure 5-9) compared to animals kept at usual culture conditions (Figure 5-8).

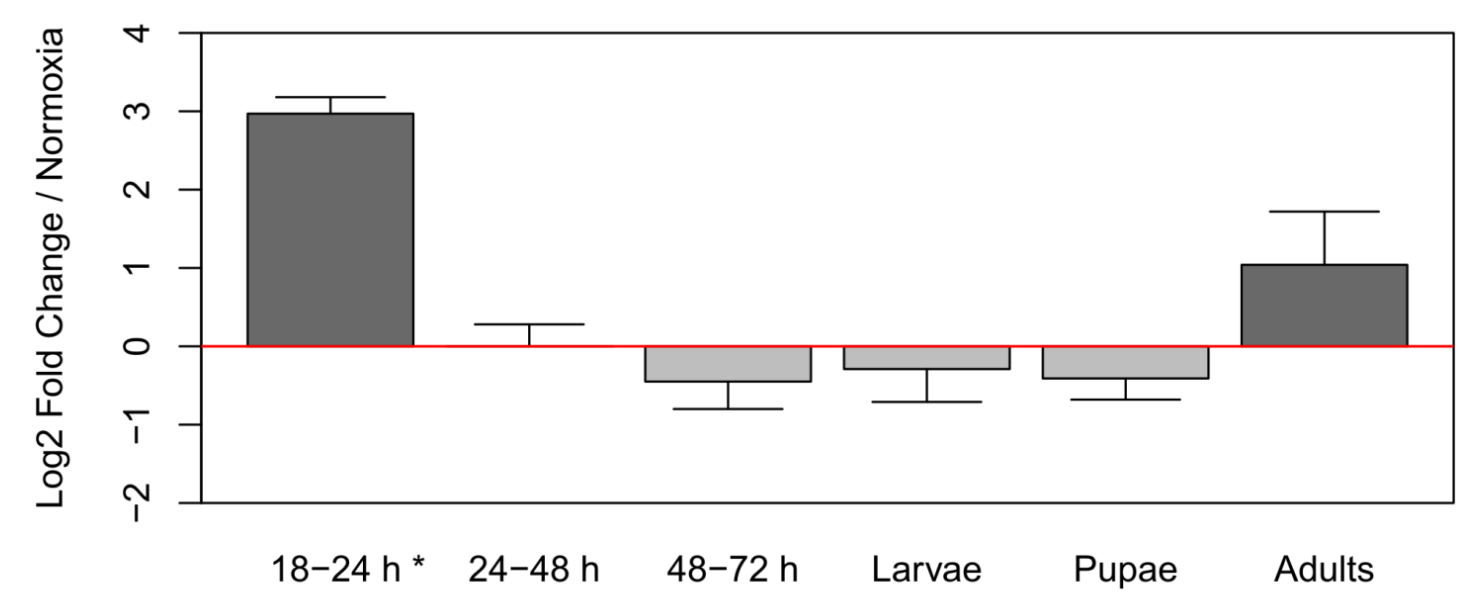

Developmental stage

Figure 5-9: Tc-crlf3 expression levels after $36 \mathrm{~h}$ hypoxia exposure in different developmental stages. Expression is normalized to the expression in control animals of the respective developmental stage kept at normoxia. Dark grey bars represent upregulation, light grey bars represent downregulation of expression. Bars show the mean and standard deviation. $\mathrm{N}=3, * \mathrm{~N}=2$.

To investigate whether expression levels are more stable when tissues are evaluated separately (tissues might react opposing to hypoxic stress), brains of larvae, pupae and adults were dissected after $36 \mathrm{~h}$ hypoxia exposure and subjected to qRT-PCR analysis (Figure 5-10). However, this approach did not eliminate variations. A tendency toward Tc-crlf3 upregulation can be noted. However, standard deviations are rather large and exceed mean values. 


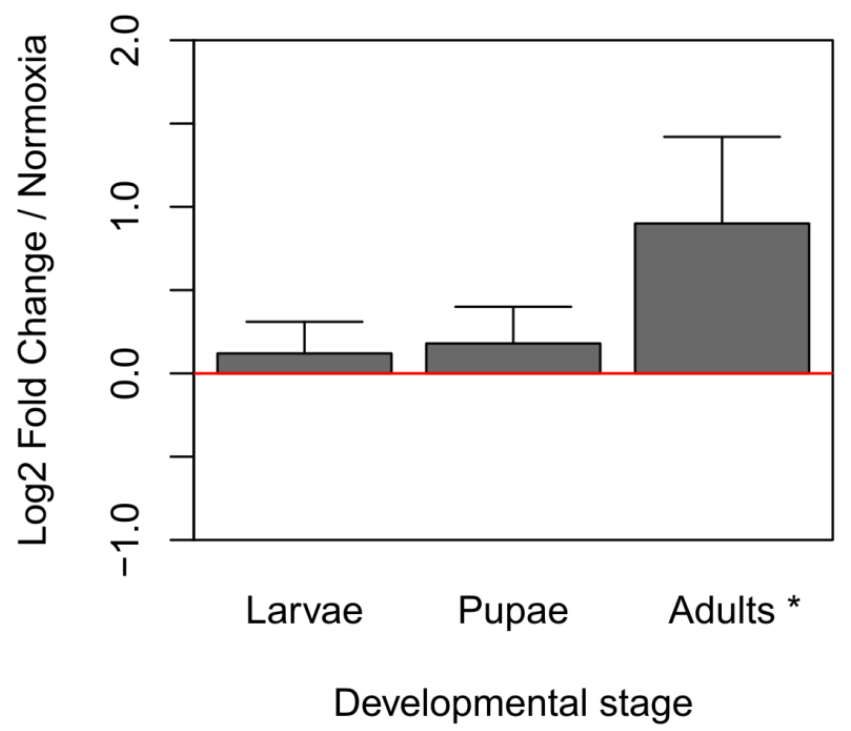

Figure 5-10: Tc-crlf3 expression levels in the brain after $36 \mathrm{~h}$ exposure to hypoxia. Expression is normalized to the expression levels in control animals of the same developmental stage kept at normoxia. Dark grey bars represent upregulation, light grey bars represent downregulation of expression. Bars show the mean and standard deviation. $\mathrm{N}=3, * \mathrm{~N}=2$. 


\subsubsection{Discussion}

Tc-crlf3 was knocked down by pRNAi resulting in a transcript reduction by $94 \%$ in $0-24 \mathrm{~h}$ old offspring embryos laid after 7 days. This strong knock-down did not affect embryo viability or larval cuticle morphology indicating that TC-CRLF3 is not crucial for embryonic or larval development in general. These results are in line with previously reported data by the iBeetle Screen (Tc number TC000209, (Dönitz et al., 2015)). The authors injected both larvae (10 animals) and pupae (10 animals) with no severe phenotypes recognizable after knock-down. It was shown that pupal injection led to $20 \%$ mortality within 11 days. Furthermore, $10 \%$ did not fulfill eclosion and another $10 \%$ succumbed as adults. After larval injection, none died within 11 days but mortality reached $10 \%$ after 22 days. For animals tested in this study, the knock-down induced reduction of Tc-crlf3 expression faded after 4 weeks. In contrast, Tc-crlf3 expression was upregulated by $64 \%$ in comparison to mock treated animals. This effect might be due to unspecific off-target effects or feedback loops (Raser and O'Shea, 2005; Scacheri et al., 2004; Singh, 2011). I assume that this is not a result of off-target effects which would have presumably led to overexpression after 7 days as well. Furthermore, we tested two non-overlapping dsRNA fragments targeting Tc-crlf3 in previous studies for soaking RNAi in cell culture (compare Manuscript 1). Our study demonstrated similar phenotypes using both fragments, arguing against off-target effects. Most likely, the observed overexpression is a result of feedback loops that control the transcriptional level retrogradely (Raser and O'Shea, 2005; Singh, 2011; Teuscher et al., 2017). To clarify this issue, it would be beneficial to furthermore evaluate the protein level. This was not possible in the past because of the lack of specific antibodies. However, future experiments will focus on TC-CRLF3 protein levels using a custom-made antibody against Tc-CRLF3 (compare subchapter 5.6.1). It might be of interest to weekly evaluate the transcription level of the female and their offspring in parallel.

Embryos of the transgenic line G10011 were used together with pRNAi embryos for in situ hybridization. G10011 embryos are identifiable by a restricted eyfp staining signal in the head and the hindgut (Figure 5-6 A). That staining was specific and did not exhibit any background staining after $19 \mathrm{~h}$. Hence, they are best suited as controls to distinguish wild-type Tc-crlf3 expressing from pRNAi embryos. Hybridization with the Tc-crlf3 probe elicits a weak and evenly distributed staining that could be misinterpreted as background staining. Though, its specificity has been shown by the addition of pRNAi embryos suffering from a strong Tc-crlf3 knock down (Figure 5-6 and Supplementary Figure 5-1, B + E) which do not exhibit any staining. Since both knock-down and wildtype Tc-crlf3 expressing embryos were fixed and stained together in the identical vial, failure of chemicals or hybridization in general can be excluded. This leads to the conclusion that Tc-crlf3 is expressed ubiquitously including neural cells in $24-48 \mathrm{~h}$ old embryos. 
In Tribolium larval brains, strong Tc-crlf3 expression is restricted to the outer rind that comprises the somata of sensory neurons. The expression pattern does not overlap with nuclear DAPI staining nor with neuropil staining by $\alpha$-Synapsin. This pattern suggests that Tc-crlf3 transcripts predominantly accumulate in the cytosol of neural cell bodies. Comparison with stainings of other studies suggests that Tc-crlf3 is expressed in neurons but not in glia (He et al., 2019; Koniszewski et al., 2016; Posnien et al., 2011). However, expression in cortex glia cannot be excluded (Hartenstein, 2011). Expression in glia could be evaluated by using transgenic reporter lines or immunohistochemistry with an $\alpha$-repo antibody (Koniszewski et al., 2016).

The quantitative evaluation showed that $T c$-crlf3 is expressed in all developmental stages to a variable extend. Similar results demonstrating developmental stage-dependent expression levels have been shown for human tissues. In many tissues, $\mathrm{Hs}$-crlf3 expression was higher in a 6 month-old embryo compared to tissues from adult individuals (Yang et al., 2009). In Tribolium, the embryonic stages and pupae express the highest amount of $T c$-crlf3 whereas larvae show the lowest abundance. Both embryos and pupae are immobile and are often deeply buried within the flour where humidity and oxygen supply varies to the surface. While Tc-crlf3 expression is stable in all three embryonic stages ( $0-24 \mathrm{~h}, 24-48 \mathrm{~h}, 48-72 \mathrm{~h})$ and pupae, it is highly variable in larvae and adults. Both are feeding stages and capable of choosing their micro environment. Hence, their need to adapt is lower than in embryos and pupae because they do not have to remain in unfavorable conditions. This might lead to a reduction of the Tc-CRLF3 receptor under unstressed conditions which might be necessary for coping with unfavorable environments such as hypoxia (Hahn et al., 2017). Furthermore, larvae and adults share the lowest degree of reorganization and development. A concomitant downregulation of genes is very frequent in larvae and adults (273 of 593) while pupae and larvae share only 1 downregulated gene (Perkin and Oppert, 2019). Larger variations of transcript levels in larvae and adults could also result from technical, rather than biological basis. Embryos are separated in $24 \mathrm{~h}$ intervals and the age of pupae is easily distinguishable. In contrast, larvae can only be roughly separated by the size of a sieve and younger and older adults are not distinguishable. It cannot be excluded that the mixture of differently aged individuals might have an impact on the Tc-crlf3 expression levels. For instance, it has been shown that the metabolic rate of adult Leptinotarsa decemlineata beetles remarkably varies independently from the body mass within the first 11 days after eclosion (Piiroinen et al., 2010). This presumably has an impact on oxygen demand and might affect the expression of Tc-CRLF3 which is seems to be necessary for coping with hypoxia (Hahn et al., 2017).

In order to investigate the effect of hypoxia on Tc-crlf3 expression, animals of each developmental stage were exposed to $36 \mathrm{~h}$ hypoxia. Some embryos and pupae were not subjected to subsequent 
RNA extraction but were placed back into flour and a climate chamber to monitor their development after hypoxia exposure. They developed to adults demonstrating that they survived hypoxia without persistent damage. Larvae and adults could be assessed directly as they are agile. The youngest embryos investigated in this assay were $18-24 \mathrm{~h}$ old at the time of RNA extraction. They were placed into hypoxia at an age of $0-12 \mathrm{~h}$ and would be $36-48 \mathrm{~h}$ old afterwards. However, the hypoxia chamber is at ambient room temperature which slows down the development by a factor of approximately half (Bucher, 2009). In this stage, Tc-crlf3 expression is upregulated by hypoxia and the response is the most pronounced amongst all stages analyzed. In addition, hypoxia might increase $T c$-crlf3 expression in adults however variations are high and prevent a firm conclusion. Expression levels are also highly variable in the remaining stages which might either be not affected by hypoxia or decrease $T c$-crlf3 expression. It is striking, that variations in the expression levels after hypoxia treatment are much bigger than in the initial experiments investigating stage-related $T c-c r l f 3$ expression. Hence, Tc-crlf3 expression might respond very sensitively and dynamically to stressors and is more stably expressed in steady-state conditions.

For decades, the hypoxia resistance of insects has been of interest in the context of pest control. Hermetic grain storage is used to minimize contamination and damage by pest insects including Tribolium. Hence, their resistance to hypoxia, anoxia and hypercapnia has been examined in many ways (Donahaye, 1990; Kharel et al., 2019; Navarro and Donahaye, 1990; Tunç and Navarro, 1983; Wang et al., 2018). Embryos are the most hypoxia-sensitive immature stage whereas pupae are the least sensitive. Exposure to $8 \% \mathrm{O}_{2}$ hypoxia for 5 days kills $47 \%$ of embryos while pupae and adults are not affected. If the exposure is prolonged to 10 days, $77 \%$ of embryos, $7 \%$ of pupae and no adults are killed (Kharel et al., 2019). It is assumed that pupae are the least hypoxia-sensitive immature stage since their metabolism is remarkably reduced during pupation. Their metabolic rate follows an U-shaped curve reaching its minimum after the first third of pupation (Kaiser et al., 2010; Odell, 1998; Park and Buck, 1960). In the blowfly, this corresponds to their most hypoxia resistant point in pupal development (Park and Buck, 1960). Larvae cope well with hypoxia, however it increases the number of molts without affecting the final body mass (Greenberg and Ar, 1996; Loudon, 1988). Adults are also well adapted to hypoxia and even survive a permanent oxygen level of $0.5 \%$. However, the mortality of adults 10 days after hypoxia exposure is significantly higher than immediately thereafter (Kharel et al., 2019). In 1990, Donahaye selected a strain resistant to low oxygen concentration (0.5\%) for 40 generations (Donahaye, 1990). It has not been elucidated in detail which adaptations occurred during the selection. One possible adaptation is the increase in tracheal tubes. In the context of this study, it would be of great interest to test $T c$-crlf3 expression in those beetles that adapted to low oxygen concentrations. 
In addition to post-hypoxia Tc-crlf3 expression in whole body samples, Tc-crlf3 expression levels were determined separately in brain tissue to focus on the central nervous system. Furthermore, this approach attempted to reduce the high variations of $T c$-crlf3 expression in biological replicates that may arise from opposing regulation in different tissues. Unfortunately, this approach did not reduce variations which hamper a clear interpretation of the data. There might be a tendency towards $T c$ crlf3 upregulation after $36 \mathrm{~h}$ hypoxia. One technical aspect that should be mentioned is the dissection of brains after hypoxia exposure. Dissections were carried out in normoxic conditions for approximately $1 \mathrm{~h}$ and might have affected the Tc-crlf3 expression level, even though animals were stored on ice to minimize artefacts.

To sum up, Tc-crlf3 expression levels in unchallenged individuals can be determined with respect to the developmental stages. However, this is more difficult in hypoxia challenged animals although the two most stable reference genes were included into the qRT-PCR analysis to increase the accuracy of relative quantitation. For future experiments, I recommend to analyze the corresponding protein levels. This has not been done in these experiments since no specific antibody was available. Therefore, a Tc-CRLF3 specific antibody has been designed (see subchapter 5.6.1) and is currently being tested. Our first results indicate that this antibody binds specifically to TC-CRLF3 so that it will be available for western blot analysis soon. In addition, I suggest examining the Tc-crlf3 expression level of the normoxic control animals in comparison to animals kept under physiological rearing conditions. The transfer of the animals from their rearing boxes in a climate chamber into the experimental set-up might induce stress due to environmental changes. Potential parameters that might have an effect could be the room temperature, the humidity, the limitation of space or starvation. It cannot be excluded that segregating the animals $36 \mathrm{~h}$ from their usual rearing conditions has an impact on the Tc-crlf3 expression level as such. 


\subsubsection{Supplementary material}

\subsubsection{Tc-crlf3 expression in the head of Tribolium embryos}

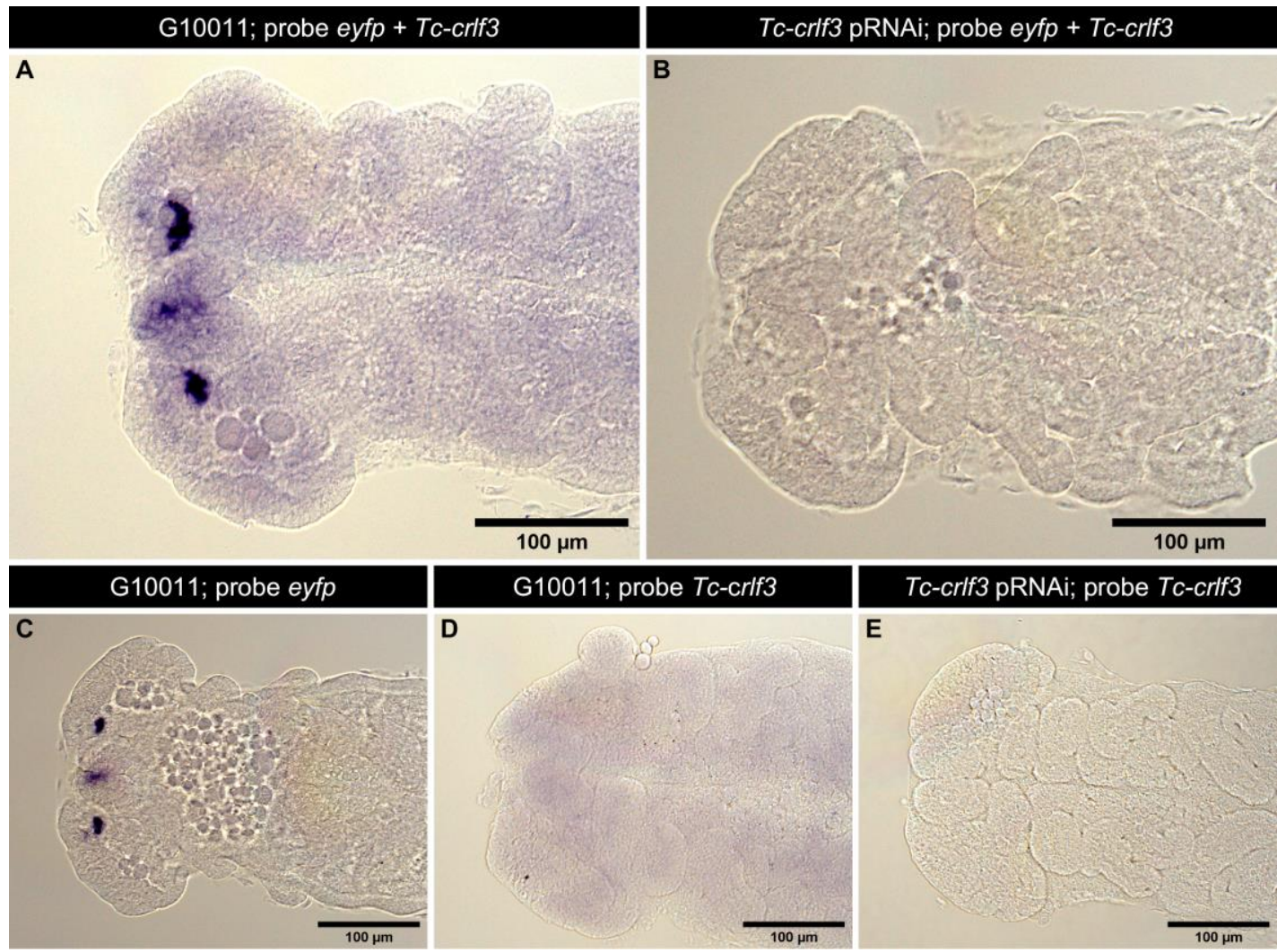

Supplementary Figure 5-1: Close ups images of $24-48 \mathrm{~h}$ old embryos stained by a NBT/BCIP color reaction of the Tc-crlf3 and eyfp (detecting egfp) probes for $19 \mathrm{~h}$ at $4^{\circ}$.C The white balance of each image is identical. (A) G10011 embryo stained with a mixture of both Tc-crlf3 and eyfp probes, (B) pRNAi embryo stained with a mixture of both Tc-crlf3 and eyfp probes, (C) G10011 embryo stained with the eyfp probe, (D) G10011 embryo stained with the Tccrlf3 probe, (E) pRNAi embryo stained with the $T c$-crlf3 probe. 


\section{$5.5 H s$-crlf3 expression in human cell lines}

\subsubsection{Introduction}

We have shown that Tribolium and locust primary brain cells are protected by human Epo and that this neuroprotective effect is mediated by the insect orthologs Tc-CRLF3 and Lm-CRLF3. Given that CRLF3 is well conserved, human CRLF3 (Hs-CRLF3) might be crucial for neuroprotection as well. This suggests CRLF3 as a neuroprotective Epo receptor that might be relevant for neuroprotective therapies treating neurodegenerative diseases. Hence, I started to investigate $\mathrm{Hs}$-CRLF3 expression in human cell lines including HEK293 (from kidney) and SH-SY5Y (neuroblastoma) cells. SH-SY5Y cells can be differentiated into dopaminergic neurons applying retinoic acid (RA) (Encinas et al., 2000; Korecka et al., 2013; Lopes et al., 2010). I aimed to investigate the Hs-crlf3 expression in different cell lines as well as its regulation upon exposure to challenging conditions and Epo treatment. SH-SY5Y cells offer the possibility to investigate Epo-mediated neuroprotection while HEK293 cells are suitable to investigate more general mechanisms of cell protection. This work was partially conducted in collaboration with Rafael Rinaldi Ferreira and Dr. Jens Gruber (German Primate Center, Goettingen, Germany). 


\subsubsection{Material and methods}

\subsubsection{Oligonucleotides}

Primers were designed exon-exon spanning to exclude amplification of genomic DNA.

Table 5-4: Summary of primers that were used for qRT-PCR analysis of human cell lines.

\begin{tabular}{|l|l|l|}
\hline Primer & Nucleotide sequence & Reference \\
\hline qHs-crlf3_Fw & 5' AAACCTGGAGGCATCATTGT 3' & Rafael Rinaldi, NM_015986 \\
\hline qHs-crlf3_Rv & 5' GCGCAGACTCTGAACTGGTA 3' & Rafael Rinaldi, NM_015986 \\
\hline qHs_Fibrillarin_Fw & 5' TGGACCAGATCCACATCAAA 3' & (Koh et al., 2011) \\
\hline qHs_Fibrillarin_Rv & 5' GACTAGACCATCCGGACCAA 3' & (Koh et al., 2011) \\
\hline qHs_GAPDH_Fw & 5' GTCAGCCGCATCTTCTTTTG 3' & (Hendig et al., 2008) \\
\hline qHs_GAPDH_Rv & 5' GCGCCCAATACGACCAAATC 3' & (Hendig et al., 2008) \\
\hline qHs_ß2M_Fw & 5' TGTGCTCGCGCTACTCTCTCT 3' & (Hendig et al., 2008) \\
\hline qHs_ß2M_Rv & 5' CGGATGGATGAAACCCAGACA 3' & (Hendig et al., 2008) \\
\hline
\end{tabular}

Hypoxia and cell differentiation often strongly affect the expression of reference genes. Hence, reference genes for experiments subjecting non-differentiated and differentiated SH-SY5Y cells to hypoxia were intensively tested for stability prior to efficiency tests. I combined the results of Delta CT, BestKeeper, Normfinder and Genorm (RefFinder Tool http://150.216.56.64/referencegene. php?type=reference) to determine the most stable reference genes. $\mathrm{Hs}$-Fibrillarin and $\mathrm{Hs}$-GAPDH were the most stable genes under hypoxia exposure and RA-induced differentiation whereas

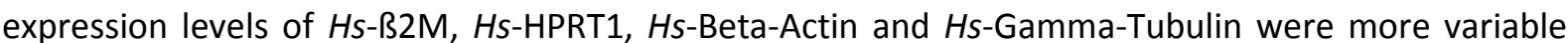
under these conditions. In HEK293 cells, Hs-ß2m was stable upon camptothecin and etoposide treatment.

\subsubsection{Cell culture}

Human embryonal kidney (HEK293) cells are cultured as described in subchapter 5.6.1.1. HeLa, SKBR3, MCF7, SW480, Jurkat and SH-SY5Y cell were cultured by Rafael Rinaldi in the Gruber Lab at $37{ }^{\circ} \mathrm{C}, 5 \% \mathrm{CO}_{2}$ in a humidified atmosphere. The respective culture media are listed in Table 5-5.

SH-SY5Y cells were split twice a week and plated into T-75 flasks or 24-well plates. They were differentiated using complete culture medium supplemented with $10 \mu \mathrm{M}$ all trans-retinoid acid 
(\#R2625, Sigma Aldrich, Germany) in DMSO for $72 \mathrm{~h}$. Their differentiation was checked by microscopy analysis for changes in morphology.

Table 5-5: Culture media for human cell lines.

\begin{tabular}{|l|l|l|l|}
\hline Cell Line & Medium & Serum & Antibiotics \\
\hline HeLa & RPMI & $10 \%$ FBS & $1 \%$ Penicillin-Streptomycin \\
\hline SKBR3 & DMEM & $10 \%$ FBS & $1 \%$ Penicillin-Streptomycin \\
\hline MCF7 & DMEM & $10 \%$ FBS & $1 \%$ Penicillin-Streptomycin \\
\hline SW480 & RPMI & $10 \%$ FBS & $1 \%$ Penicillin-Streptomycin \\
\hline Jurkat & RPMI & $10 \%$ FBS & $1 \%$ Penicillin-Streptomycin \\
\hline SH-SY5Y & DMEM F-12 & $10 \%$ FBS & $1 \%$ Penicillin-Streptomycin \\
\hline
\end{tabular}

\subsubsection{Hypoxia and pharmacological treatment}

SH-SY5Y cells were incubated for $24 \mathrm{~h}$ in hypoxia $\left(\mathrm{O}_{2}\right.$ level $\left.\leq 1 \%\right)$ at $37^{\circ} \mathrm{C}$ in $\mathrm{L} 15$ medium (Leibovitz's L15 Medium, \#11415049, Thermo Fisher Scientific, Germany) without FBS. L15 medium replaced DMEM because DMEM requires $\mathrm{CO}_{2}$ supply which was not available under hypoxic conditions. Normoxic control cells were incubated in parallel with DMEM F-12 without FBS as described above. HEK cells were plated 1:10 before each treatment. Furthermore, cells were pharmacologically stressed by addition of either camptothecin or etoposide. Camptothecin (10 $\mu \mathrm{M}$, stock solution $1 \mathrm{mM}$ in DMSO, \#C9911, Sigma Aldrich, Germany) was applied on day 4 for $4 \mathrm{~h}, 7 \mathrm{~h}$ or $24 \mathrm{~h}$. Etoposide (20 $\mathrm{\mu M}$, stock solution 5 mM in DMSO, \#E1383, Sigma Aldrich, Germany) was applied on day 3 for $30 \mathrm{~min}$ or $4 \mathrm{~h}$. Epo ( $84 \mathrm{ng} / \mu \mathrm{l}$, NeoRecormon, Roche, Switzerland) was applied $18 \mathrm{~h}$ prior to etoposide treatment.

\subsubsection{RNA extraction}

SH-SY5Y cells were homogenized with TRIzol Reagent (\#15596026, Thermo Fisher Scientific, Germany). Total RNA was extracted according to the user manual with chloroform, precipitated with isopropanol, washed with ethanol and resuspended in $20-30 \mu \mathrm{l} \mathrm{H}_{2} 0$. Subsequently, total RNA (1 $\left.\mu \mathrm{g}\right)$ was transcribed into cDNA using the SensiFAST cDNA Synthesis Kit (\#BIO-65053, Bioline Reagents Ltd, United Kingdom).

Total RNA from HEK293 cells was purified using the Monarch ${ }^{\circledR}$ Total RNA Miniprep Kit (\#T2010S, New England BioLabs ${ }^{\circledR} \mathrm{GmbH}$, Germany) according to the user manual including the recommended oncolumn DNAse I treatment and finally eluted in $30 \mu \mathrm{dddH_{2 }} O$. cDNA was synthesized from $1 \mu \mathrm{g}$ total 
RNA using the QuantiTect Reverse Transcription Kit (\#205311, Qiagen, Germany) according to the user manual.

\subsubsection{Statistics}

Expression levels of biological replicates are shown as the mean with the standard deviation. Statistical analysis was not performed due to the small number of biological replicates. 


\subsubsection{Results}

\subsubsection{Hs-crlf3 expression in human cell lines}

We have examined seven human cell lines for endogenous Hs-crlf3 expression (Figure 5-11). In comparison to expression in HEK293 cells (set to 1), HeLa cells (0.09, human cervix adenocarcinoma) express the lowest amount of Hs-crlf3 followed by SKBR3 $(0.29$, human breast cancer), SW480 (0.46, human colon cancer), MCF7 (0.53, human breast cancer) and Jurkat (0.55, human T-lymphocyte) cells. HEK293 (1, human embryonic kidney) and SH-SY5Y (1.53, human neuroblastoma) cells express the highest amounts of Hs-crlf3. This evaluation was only performed once in order to screen for interesting cell lines. HEK293 and SH-SY5Y cells were chosen for further analysis.

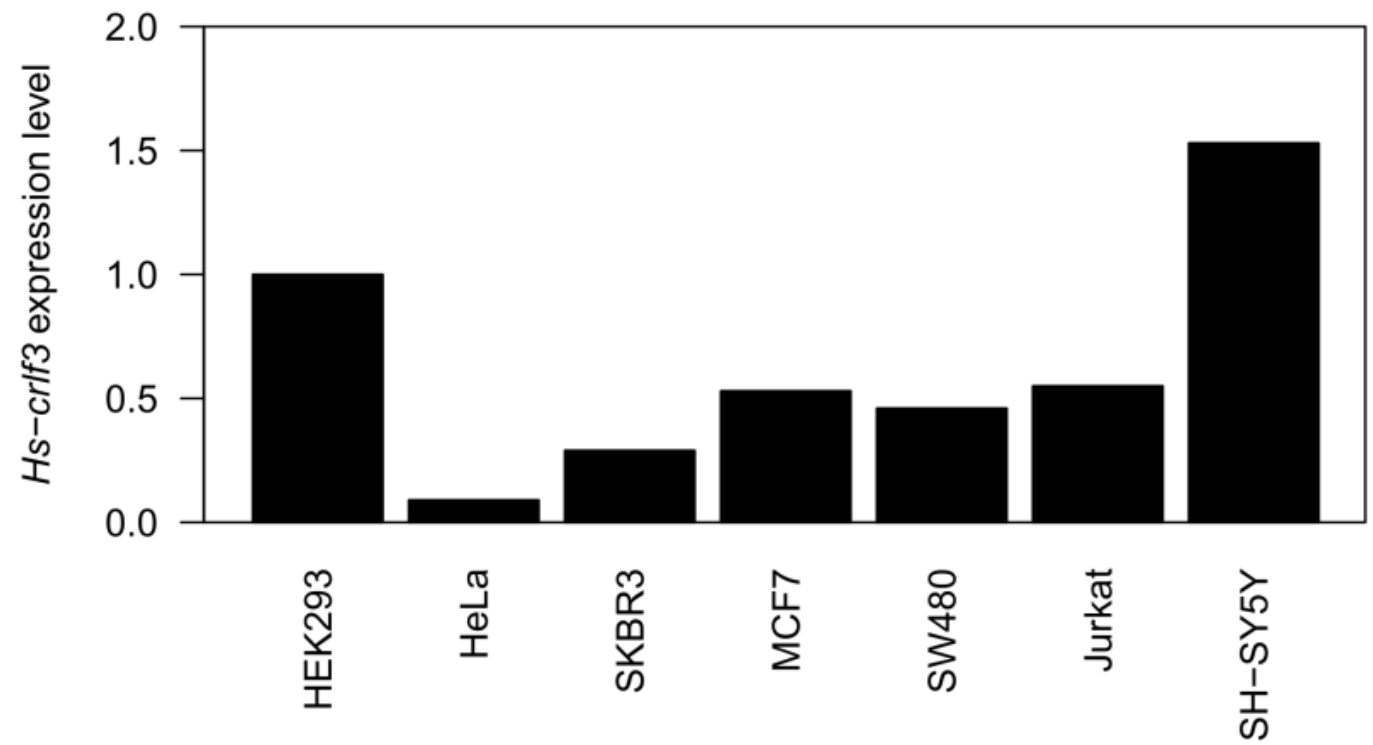

Cell line

Figure 5-11: Hs-crlf3 expression in various human cell lines. Evaluation of HEK293 (human embryonic kidney), HeLa (human cervix adenocarcinoma), SKBR3 (human breast cancer), MCF7 (human breast cancer), SW480 (human colon cancer), Jurkat (human T-lymphocyte) and SH-SY5Y cells (human neuroblastoma). Reference gene $H s-\beta 2 M . N=1$.

\subsubsection{Hs-crlf3 expression in SH-SY5Y cells}

In order to investigate the regulation of $\mathrm{Hs}$-crlf3 expression in a human neuron-like model system, SH-SY5Y and differentiated SH-SY5Y cells were exposed to 24 h hypoxia. Hypoxia reduces $H s$-crlf3 in non-differentiated (0.49 \pm 0.03 vs. 1$)$ and differentiated SH-SY5Y cells ( $0.7 \pm 0.1$ vs. $1.65 \pm 0.2)$ compared to normoxic control groups (Figure 5-12). In both normoxia and hypoxia differentiated SHSY5Y cells express higher amounts of Hs-crlf3 than non-differentiated SH-SY5Y cells. 


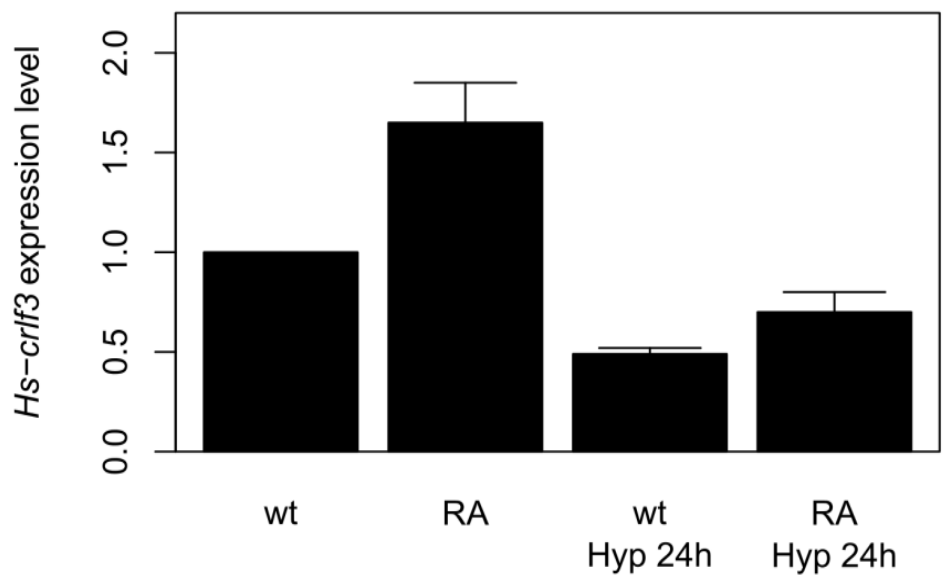

Figure 5-12: Hs-crlf3 expression in differentiated (RA) and non-differentiated (wt) neuroblastoma SH-SY5Y cells upon $24 \mathrm{~h}$ hypoxia exposure. Hypoxia decreases $\mathrm{Hs}$-crlf3 expression while differentiation into neurons increases Hs-crlf3 expression. RA retinoic acid, wt wild-type. Reference genes Hs-Fibrillarin and Hs-GAPDH. Bars show the mean \pm standard deviation, $\mathrm{N}=4$.

\subsubsection{Hs-crlf3 expression in HEK293 cells in response to camptothecin, etoposide and Epo}

In addition, HEK293 cells were examined for their response to Epo and chemical stress. Hs-crlf3 expression was reduced by treatment with $10 \mu \mathrm{M}$ camptothecin for $4 \mathrm{~h}(0.64 \pm 0.08)$, for $7 \mathrm{~h}(0.28 \pm$ $0.0)$ and for $24 \mathrm{~h}(0.3 \pm 0.05)$ (Figure 5-13). Similar experiments were performed using etoposide $(20 \mu \mathrm{M})$ to induce apoptosis (Figure 5-14). After treatment for $30 \mathrm{~min}(0.85 \pm 0.17)$ and $4 \mathrm{~h}(0.87 \pm$ 0.2) Hs-crlf3 expression tends to be slightly reduced as well. If HEK293 cells were pre-treated $18 \mathrm{~h}$ prior to $30 \mathrm{~min}$ etoposide exposure with $84 \mathrm{ng} / \mu \mathrm{l} \mathrm{Epo}$, this tended to increase $\mathrm{Hs}$-crlf3 expression $(1.08 \pm 0.3)$. Epo treatment for $18.5 \mathrm{~h}(18 \mathrm{~h}$ pre-treatment $+30 \mathrm{~min})$ alone showed a similar expression level $(1.07 \pm 0.2)$. However, if cells were treated for $22 \mathrm{~h}$ (18 h pre-treatment $+4 \mathrm{~h}$ ) with Epo $(1.12 \pm 0.1)$, an increase in Hs-crlf3 upregulation got more apparent. In contrast, $18 \mathrm{~h}$ pretreatment with $4 \mathrm{~h}(0.82 \pm 0.18)$ of etoposide exposure led to a downregulation of $\mathrm{Hs}$-crlf3 expression. Thus, pre-treatment with Epo before $30 \mathrm{~min}$ exposure to $20 \mu \mathrm{M}$ etoposide tends to upregulate $H s$-crlf3 expression while it downregulates the expression after $4 \mathrm{~h}$ etoposide exposure. 


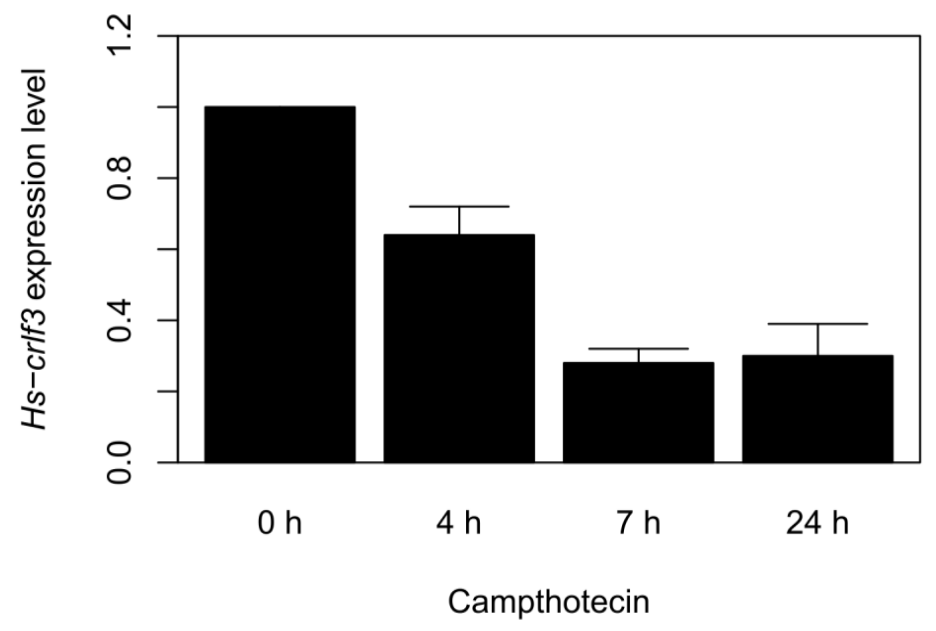

Figure 5-13: Hs-crlf3 expression in HEK293 cells treated with camptothecin. Cells were treated with $10 \mu \mathrm{M}$ camptothecin for $4 \mathrm{~h}, 7 \mathrm{~h}$ and $24 \mathrm{~h}$. Reference gene $H s-\beta 2 M$. Bars show the mean \pm standard deviation. $\mathrm{N}=3$.

$30 \mathrm{~min}$

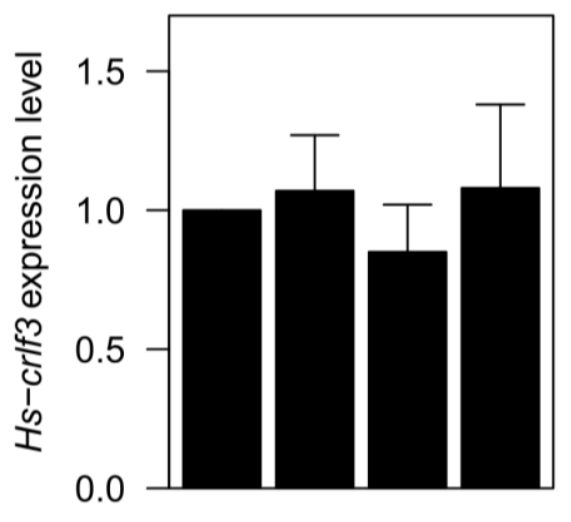

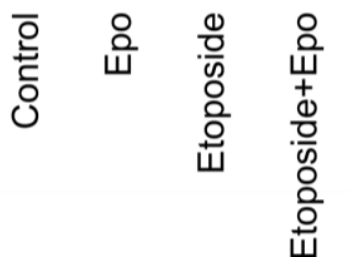

$4 \mathrm{~h}$

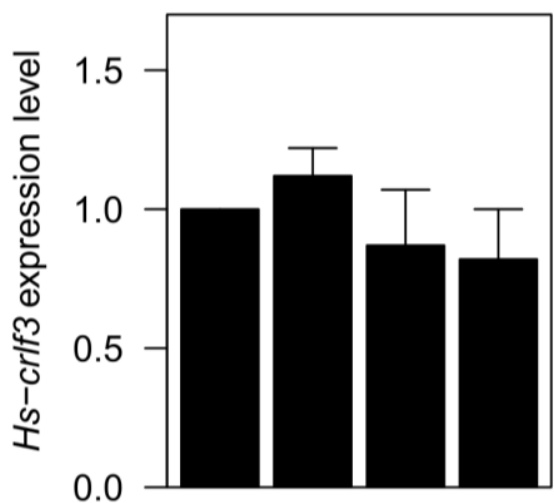

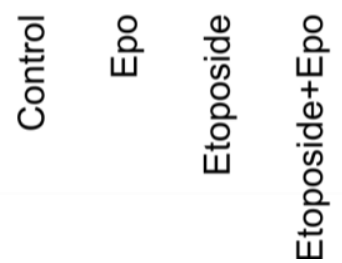

Figure 5-14: Hs-crlf3 expression in HEK293 cells treated with Epo and etoposide. Epo (84 ng/ $\mathrm{ll}$ ) was applied $18 \mathrm{~h}$ prior to etoposide $(20 \mu \mathrm{M})$ treatment. Expression was examined $30 \mathrm{~min}$ and $4 \mathrm{~h}$ after etoposide treatment. Reference gene Hs- $\beta 2 M$. Bars show the mean \pm standard deviation. $\mathrm{N}=3$. 


\subsubsection{Discussion}

Hs-crlf3 is expressed in various human cell lines, however highest expression was detected in HEK293 and SH-SY5Y cells. Its expression has been detected before in human cell lines, human tissue (e.g. brain, liver, spleen, and pancreas) and freshly dissected tumor tissue (Yang et al., 2009). Here, I demonstrate that Hs-crlf3 expression is high in neuroblastoma derived SH-SY5Y cells and is further increased by their differentiation into neurons. The treatment of SH-SY5Y with RA results in dopaminergic neurons that are a common model system for Parkinson's disease studies (Lopes et al., 2010). RA is crucial for the developing nervous system regulating neural patterning and neuronal differentiation but also for axon regeneration in adults (Maden, 2007). The markedly high expression in differentiated, neuron-like SH-SY5Y cells might indicate an important role of Hs-CRLF3 in neurons.

HEK293 and SH-SY5Y cells downregulate Hs-crlf3 expression upon apoptotic stimuli (Figure 5-13 and Figure 5-14). SH-SY5Y cells were stressed with hypoxia whereas HEK293 cell were chemically stressed with camptothecin and etoposide. Camptothecin and etoposide induce apoptosis by causing DNA double-strand breaks (Muslimovic et al., 2009; Yamauchi et al., 2011). Apoptosis induction with camptothecin down regulated Hs-crlf3 in dependence of the exposure time. A similar trend was detected with etoposide but it was not as pronounced (Figure 5-14). The concentration might be too low or the exposure time to short. Both concentration and exposure time have previously been selected on the basis of morphological changes upon etoposide treatment (results are shown in subchapter 5.6.1.3)

A downregulation of $\mathrm{Hs}$-crlf3 transcripts might seem conflicting at first sight but the following aspects should be taken into account. Protein levels can be controlled at various levels. For instance, transcription activity can be repressed or enhanced and the stability of mRNAs can be altered. Furthermore, transcripts can be stored in stress granules to protect them against exosomal degradation (Anderson and Kedersha, 2006; Guarino et al., 2019; Thomas et al., 2011). In addition, translation can be modulated by differential usage of ribosomes (Lee et al., 2011; Liu et al., 2016). The protein level itself can by altered by modifying the stability and degradation of proteins (Lackner et al., 2012). Taking all these factors into account, it is obvious that the mRNA level alone does not determine the protein abundance.

The average correlation between mRNA and protein levels is about $40 \%$ (De Sousa Abreu et al., 2009; Gygi et al., 1999; Schwanhäusser et al., 2011). The Pearson's correlation coefficient is best in yeast $(0.34-0.87)$, worse in bacteria $(0.2-0.47)$ and the worst in multicellular organisms $(0.09-$ 0.46) including human tissues (reviewed in (De Sousa Abreu et al., 2009)). In yeast, it has been shown that particular protein levels can vary up to 20 -fold while the corresponding mRNA level remains 
stable. Vice versa, mRNA levels can vary up to 30 -fold without affecting the protein level (Gygi et al., 1999). Best correlations were achieved in cells and tissues under steady-state conditions. Stress, changes in growth rate, starvation or other perturbed phases dramatically decrease the correlation between mRNA and protein level due to higher variations of post-transcriptional processes (Liu et al., 2016). For instance, mRNA turn-over slows down during stress. If bacteria are stressed by glucose deprivation, the median mRNA half-live almost doubles (Esquerré et al., 2014). Moreover, it has been shown that stress-induced growth arrest leads to a pronounced decrease of bulk mRNA but only slightly reduces the protein abundance (Marguerat et al., 2012). Additionally, mRNA-to-protein correlation is stronger in upregulated genes than in down-regulated genes which exhibit only a poor correlation (Lee et al., 2011). The biological reason might be that regulatory proteins are necessarily encoded by short-lived transcripts and that proteins with long half-lives far exceed the presence of their respective mRNA transcripts. In general, protein half-lives are longer than mRNA half-lives (Meyer et al., 2004). In mammals, the transcript half-lives range from $15 \mathrm{~min}$ to more than $24 \mathrm{~h}$ (Shyu et al., 1989). Schwanhäusser and colleagues reported a median half-live of $9 \mathrm{~h}$ for fibroblast transcripts and a median protein half-life of $46 \mathrm{~h}$ in mice (Schwanhäusser et al., 2011). However, certain proteins were retained for more than $200 \mathrm{~h}$.

The half-life and turnover rate of CRLF3 are unknown. However, it might be similar to those of other group 1 class I cytokine receptor members (EpoR, thrombopoietin receptor (TpoR), prolactin receptor (PrIR) and growth hormone receptor (GhR)) (Liongue and Ward, 2007). The half- life of EpoR in murine cells has been reported to be $45-60$ min which is similar to the half-life of GhR (30-45 min) (Baxter, 1985; Gorin and Goodman, 1985; Neumann et al., 1993; Yoshimura et al., 1990). Nevertheless, GhR residues were still detectable by western blot staining after 160 min (Van Kerkhof et al., 2000). This shows that degradation does not proceed linearly and that knowledge about the turnover rates would be even more informative. Reports of the PrlR half-life differ from $40-50$ min up to 80 min (Baxter, 1985; Genty et al., 1994). Stimulation by the ligand tremendously reduced the half-life at the cell surface to $8 \mathrm{~min}$ (Genty et al., 1994). For TpoR, a half-life of $5 \mathrm{~h}$ and a replacement of mature receptors at the cell surface approximately every $6 \mathrm{~h}$ are reported (Pecquet et al., 2012; Saur et al., 2010). The stability of TpoR is exceptionally long also in comparison to other cytokine receptors such as the common gamma chain $\gamma_{c}$ (Royer et al., 2005).

For all of the group 1 class I cytokine receptors, it has been shown that only a minor portion of mature protein is present at the cell surface and that the majority is stored in internal pools (Becker et al., 2010; Dahlen et al., 2003; Genty et al., 1994; Leung et al., 1997). Intracellular pools allow a prompt replacement of ligand-activated receptors, which are internalized from the cell surface, without the need for de novo synthesis. This mechanism represents the first level of regulation to 
maintain or enhance cellular sensitivity for a cytokine ligand and only afterwards transcription and translation would be required to refill the storage pool of cytokine receptors. Thus, it might be of importance to additionally investigate the expression levels during shorter intervals as well as longer intervals of hypoxia. It cannot be excluded that during hypoxia $\leq 36 \mathrm{~h}$ CRLF3 receptors at the cell surface are replaced by pre-existing ones stored in internal pools since the average half-life of human proteins is $46 \mathrm{~h}$ (ranging to $200 \mathrm{~h}$ and more) (Schwanhäusser et al., 2011). In addition, it will be useful to investigate crlf3 expression levels in post-hypoxic intervals determining whether transcription bursts afterwards in order to refill these internal pools as soon as energy is available again. It has been demonstrated that translation can be reduced by $50 \%$ during hypoxia but recovers to normal levels within $1 \mathrm{~h}$ of reoxygenation (Lang et al., 2002). In general, hypoxia leads to a reduction of translation and transcription due to the lack of ATP availability, since both processes require energy (Cavadas et al., 2017; Liu and Simon, 2004). Therefore, hypoxia-exposed cells may compensate reduced production of protein by reduction of protein degradation, which also involves energyconsuming processes (Lackner et al., 2012).

Strikingly, Epo had a tendency to upregulate $H s$-crlf3 expression in combination with 30 min etoposide treatment but decreases $\mathrm{Hs}$-crlf3 expression after $4 \mathrm{~h}$. This might be due to desensitization in order to protect the cell from overstimulation. After $4 \mathrm{~h}$, there might be a higher amount of the endogenous Hs-CRLF3 ligand as a response to chemical stress by etoposide. This might, in combination with the artificial Epo stimulation, result in a high amount of potential ligands. One mechanism of desensitization is the down-regulation of transcription and has been demonstrated for various receptors (Bouvier et al., 1989; Koerte et al., 2018; Pype et al., 1998; Rousell et al., 1995; Saucier and Albert, 1997).

To further investigate the presence of Hs-CRLF3 in response to stressful stimuli and Epo treatment in detail, evaluation of the protein level will be helpful. However, the knowledge about the dynamics at the transcriptional level provided herein is inevitable to comprehensively understand the full process of Hs-CRLF3 regulation. 


\subsection{Tools}

Besides the publication of two peer-reviewed journal articles, the preparation of a third manuscript and the profound evaluation of insect as well as human crlf3 expression, I additionally designed and established further tools that will be substantial for future experiments characterizing CRLF3 orthologs. I established a robustly working protocol for TUNEL staining of HEK293 cells to determine the cell viability by flow cytometry. This cell viability assay is essential for future studies investigating the cell protective effect of Epo and the involvement of Hs-CRLF3 in human cells. Furthermore, I designed a custom-made antibody against Tc-CRLF3 to enable the detection of proteins which will additionally complement the herein presented results of the Tc-crlf3 expression level. Its specificity has already been proven. Moreover, I implemented a new model system to study and characterize the cell protective function of Tc-CRLF3 in more detail by expressing an eGFP-tagged Tribolium CRLF3 receptor in Drosophila S2 cells. The following subchapter describes these tools. 


\subsubsection{Cell viability assay with HEK293 cells}

I demonstrated that CRLF3 orthologs in the beetle Tribolium and the locust are crucial for Epoinduced neuroprotection. In order to support the development of safe neuroprotective therapies, it is indispensable to investigate CRLF3 as a cell protective Epo-receptor in human cells. On the one hand, I investigated CRLF3 expression in human cell lines (see subchapter 5.5). On the other hand, I started to establish cell viability assessment methods in our lab using human embryonal kidney (HEK293) cells. HEK293 cells are a common in vitro model system, easy to handle and should not express the classical Epo receptor EpoR (Heir et al., 2016; Ott et al., 2015). Even though it is not possible to investigate neuroprotective effects in HEK293 cells, they still offer the possibility to investigate common cell protective signaling pathways. It has been shown that the kidney is sensitive to Epo treatment inducing tissue protection in vivo (Moore and Bellomo, 2011; Ozkurt et al., 2018). Here, I provide the basis for future experiments investigating the cell protective effect of Epo in HEK293 cells. I established TUNEL (Terminal deoxynucleotidyl transferase dUTP nick end labeling) assay as well as cell cycle analysis in order to evaluate cell viability after etoposide treatment for apoptosis induction.

\subsubsection{HEK293 cell maintenance}

HEK293 cells were cultured in DMEM (\#41965-039, Thermo Fisher Scientific, Germany) and 10 \% FBS superior (not heat inactivated, \#S 0615, Biochrom, Germany) at $37{ }^{\circ} \mathrm{C}$ in a humidified atmosphere containing $5 \% \mathrm{CO}_{2}$. For maintenance, HEK293 cells were split up to 1:10 twice a weak. Therefore, cells in a confluent flask were rinsed twice with PBS and incubated with $1 \mathrm{ml} 0.25 \%$ Trypsin-EDTA (\# 25200056, Thermo Fisher Scientific, Germany) for $1-2$ min at $37^{\circ} \mathrm{C}$. When the cells started to detach, trypsin activity was stopped by addition of $4 \mathrm{ml}$ culture medium. Cells were washed, dissociated and collected in a $15 \mathrm{ml}$ conical vial. They were spun down (500 g, $5 \mathrm{~min}, \mathrm{RT})$ and the supernatant was discarded. The cell pellet was resuspended in $1 \mathrm{ml}$ culture medium and seeded into a new T25 flask. The cells were cultured up to passage P50. Frozen stocks were prepared and thawed as described for Drosophila S2 cells (see subchapter 5.6 .3 ) but with a different freezing medium (10 \% sterile DMSO and $10 \%$ FBS in DMEM).

\subsubsection{TUNEL staining in HEK293 cells}

Viability of HEK293 cells was determined by DNA fragmentation analysis utilizing the TUNEL Assay Kit - FITC (\#ab66108, Abcam, United Kingdom). HEK293 cells in a confluent T25 flask were mechanically detached with a pasteur pipette and strained through a $40 \mu \mathrm{m}$ cell strainer into a $15 \mathrm{ml}$ conical vial. Cells were pelleted at $300 \mathrm{~g}$ for $5 \mathrm{~min}$ at $4{ }^{\circ} \mathrm{C}$ and the supernatant was carefully removed with a pasteur pipette. The pellet was resuspended in $500 \mu \mathrm{lPBS}(\mathrm{pH}$ 7.4) before dropwise adding $5 \mathrm{ml}$ of $1 \%$ PFA and vortexing. During fixation on ice for $15 \mathrm{~min}$, the vials were swirled from time to time to 
prevent cell aggregation. Afterwards, cells were washed two times in PBS ( $300 \mathrm{~g}$ for $5 \mathrm{~min}$ at $4{ }^{\circ} \mathrm{C}$ ) before the cell pellet was resuspended in $500 \mu$ PBS. To permeabilize the cells, they were added to $5 \mathrm{ml}$ of ice-cold $70 \%$ ethanol while shaking and stored at $-20^{\circ} \mathrm{C}$ for at least $30 \mathrm{~min}$. After permeabilization, all centrifugation steps were performed at $600 \mathrm{~g}$ for 5 min at $4{ }^{\circ} \mathrm{C}$.

For each sample, $2 \mathrm{ml}$ cell suspension were pelleted in a FACS tube (\#352052, Falcon ${ }^{\circledR}$, Corning Life Science, United States of America) and washed twice with PBS. The DNA Labeling solution was prepared and applied at $37^{\circ} \mathrm{C}$ in the dark for 60 min according to the user manual. During the incubation, the cells were swirled every $15 \mathrm{~min}$ to ensure even staining. Subsequently, cells were washed twice with rinse buffer, resuspended in $500 \mu$ propidium iodide/RNAse A solution and incubated from $30 \mathrm{~min}$ to $3 \mathrm{~h}$ in the dark at RT. Unstained cells were kept in PBS (pH 7.4) supplemented with $2 \mathrm{mM}$ EDTA and stored at $4{ }^{\circ} \mathrm{C}$ until measurements. Samples were analyzed with a BD LSR II flow cytometer and the BD FACSDiva software (version 6.1.3) using the $488 \mathrm{~nm}$ laser and the AF488 (detecting FITC) and PerCP (detecting PI) filters. FITC- labeling represents apoptotic cells whereas PI staining was used as counterstaining and for cell cycle analysis.

\subsubsection{Induction of apoptosis using etoposide}

In order to induce apoptosis in HEK293 cells, cells were treated with etoposide (stock $5 \mathrm{mM}$ dissolved in DMSO). Cells were passaged 1:10 two days before treatment and different concentrations of etoposide were tested ranging from $10 \mu \mathrm{M}$ to $30 \mu \mathrm{M}$. Figure 5-15 shows the morphological assessment of cell death for incubation periods ranging from $24 \mathrm{~h}$ to $48 \mathrm{~h}$. After $24 \mathrm{~h}$, cells seem to be stressed when exposed to $\geq 20 \mu \mathrm{M}$ etoposide. In contrast, after $48 \mathrm{~h}$ even $10 \mu \mathrm{M}$ etoposide seem to stress the cells. In the following experiments, $20 \mu \mathrm{M}$ etoposide treatments for at least $24 \mathrm{~h}$ were chosen for cell viability assessment by flow cytometry and for analysis of stress-dependent CRLF3 expression in HEK293 cells. 


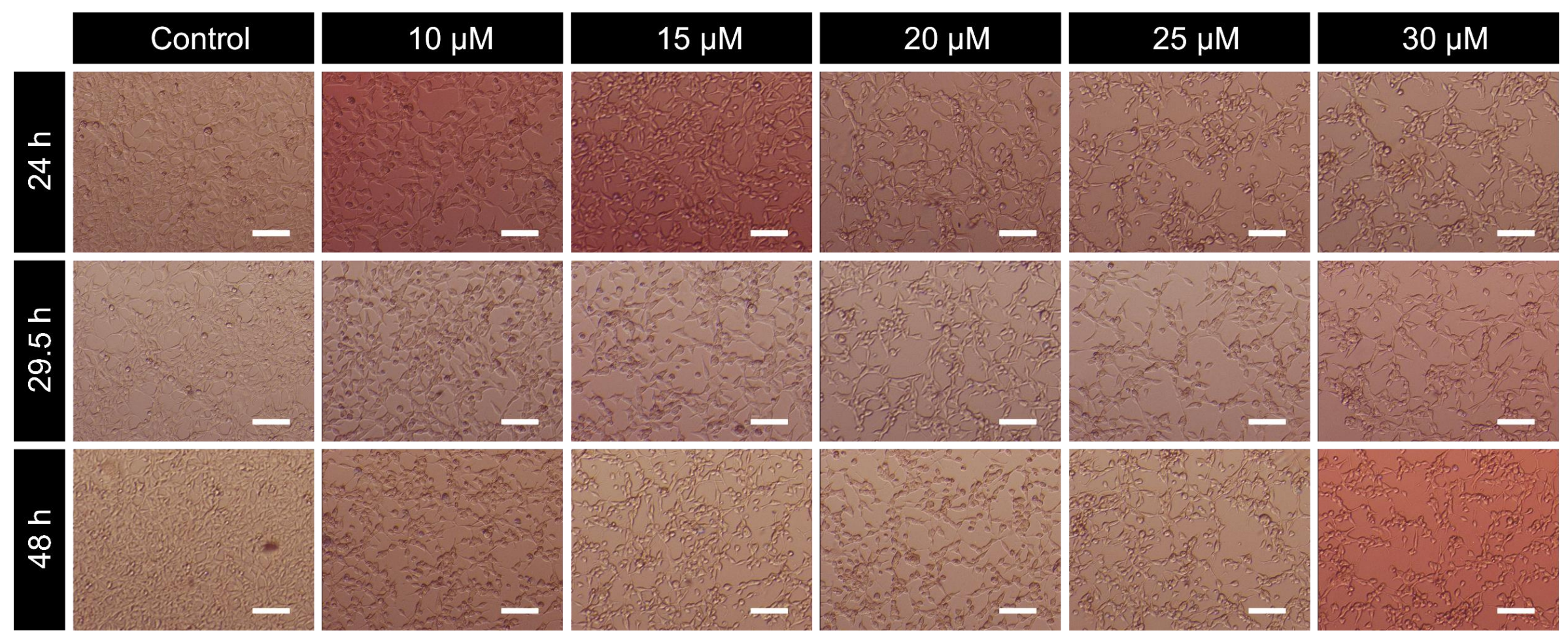

Figure 5-15: Morphological changes of HEK293 cells after induction of apoptosis using different concentrations of etoposide (10 $\mu \mathrm{M}$ to $30 \mu \mathrm{M})$. Stressed cells stop proliferating, detach and appear round instead of stretched. Scale bar $100 \mu \mathrm{M}$. 


\subsubsection{Cell viability assessment after etoposide and Epo treatment}

The cell viability assay aimed to investigate whether Epo protects HEK293 from etoposide-induced apoptosis. This experiment is the basis for future studies examining the involvement of human CRLF3 in Epo-induced cell protection. Hence, HEK293 cells were pre-treated with $84 \mathrm{ng} / \mathrm{ml}$ rhEpo ( $\hat{=}$ $10 \mathrm{U} / \mathrm{ml}) 18 \mathrm{~h}$ before they were stressed with $20 \mu \mathrm{M}$ etoposide. In order to assess cell viability, the cells were subjected to TUNEL staining and analyzed with a BD LSRII flow cytometer using the $488 \mathrm{~nm}$ laser, the AF488 filter and the PerCP filter.

The flow cytometric analysis of the TUNEL assay with additional propidium iodide counterstaining is shown in Figure 5-16 A-B. Etoposide treatment (final concentration $20 \mu \mathrm{M}$ ) for $26 \mathrm{~h}$ increases the population of TUNEL positive stained apoptotic cells to $11.2 \%$ (non-treated control $2.63 \%$, not shown). In contrast, only $6.4 \%$ HEK293 cells are TUNEL positive when additionally pre-treated with $84 \mathrm{ng} / \mathrm{ml}$ Epo. This indicates that Epo protects HEK293 cells from etoposide-induced apoptosis. Moreover, the cell viability of these samples was also evaluated on the basis of propidium iodide staining (detecting DNA) and cell cycle analysis. Figure 5-16 C shows the gating of single cells which is essential for cell cycle analysis. Only single cells were used for cell cycle analysis shown in Figure 5-16 D. The histogram of the control sample (grey) distinctly depicts the cell cycle consisting of the G1, synthesis (S) and G2 phase. Treatment with etoposide (red and light red) increased a population with less DNA than in the G1 phase. This peak is called Sub-G1 (indicated by the arrow) and corresponds to apoptotic cells whose DNA has already been cleaved (Darzynkiewicz et al., 1997). The population of cells in the Sub-G1 peak should correlate with the number of apoptotic cells determined by TUNEL assay (Figure 5-16 A, B). However, the minor difference (6.4 \% vs. $11.2 \%$ ) between apoptotic cells in additionally Epo-treated and exclusively etoposide-treated cells is only weakly distinguishable. Nevertheless, cell cycle analysis reveals that etoposide and Epo treatment does not only affect apoptosis but also the cell cycle. Etoposide treatment increases the population of cells in S phase and to some extent in G1 phase. In contrast, it remarkably reduces the population of cells in the G2 phase. This shift into S phases and later G2 phase is common since etoposide is a topoisomerase II inhibitor that interferes with chromosome segregation during mitosis (Chen et al., 1984; Clifford et al., 2003; Cortés et al., 2003; Selvarajah et al., 2015). It further underlines the proper application of the cell cycle assay. 
A

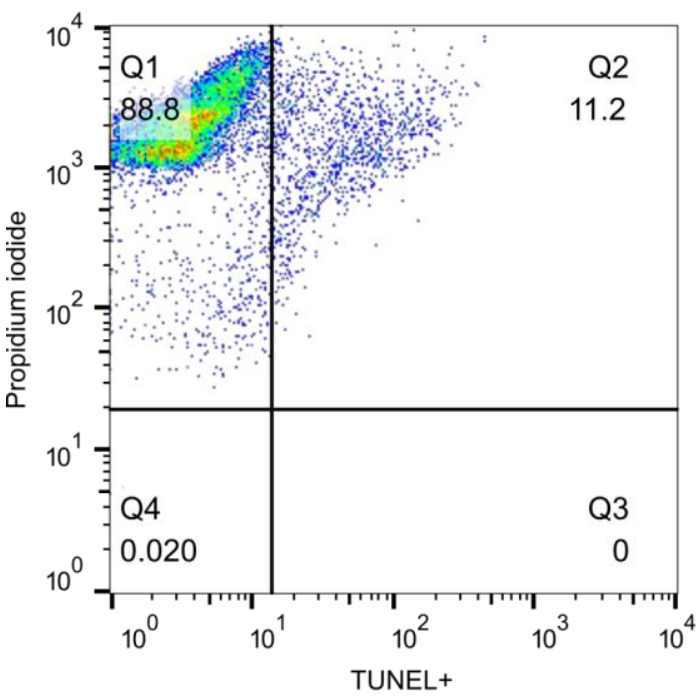

C

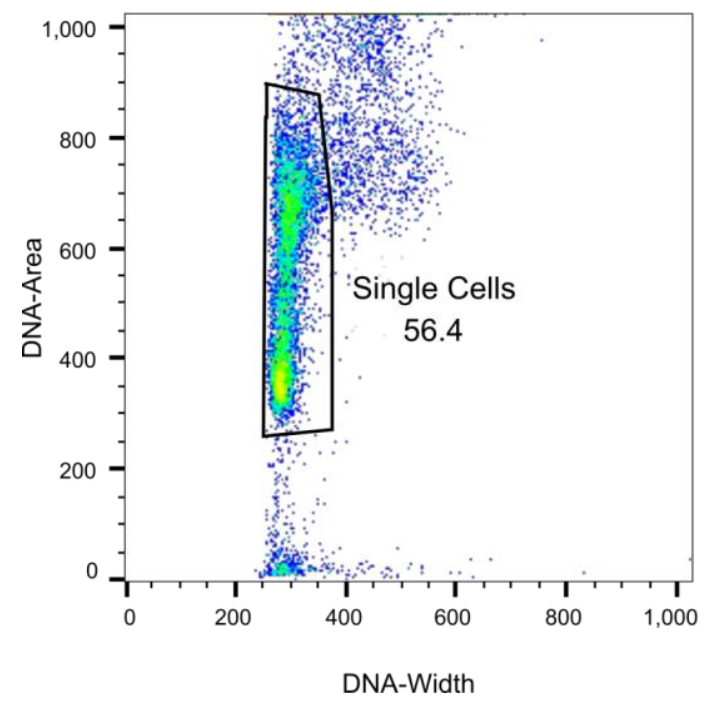

B

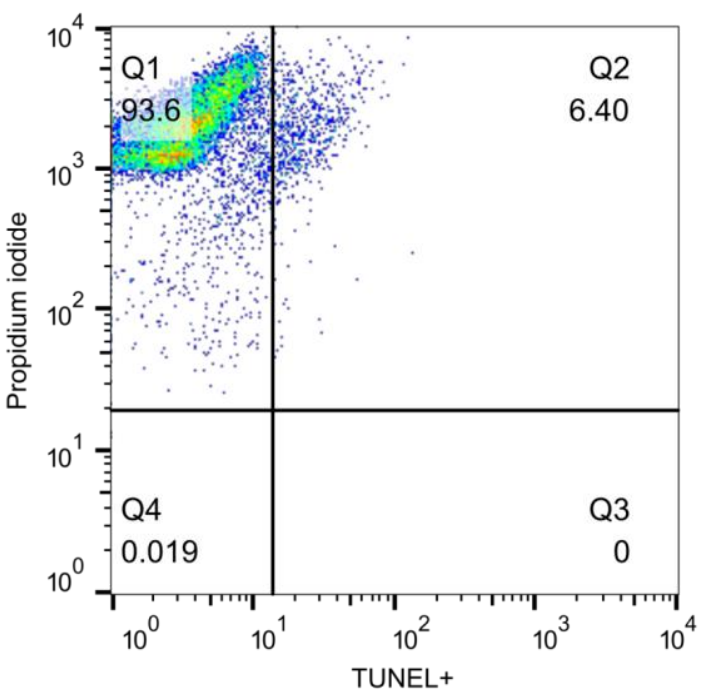

D

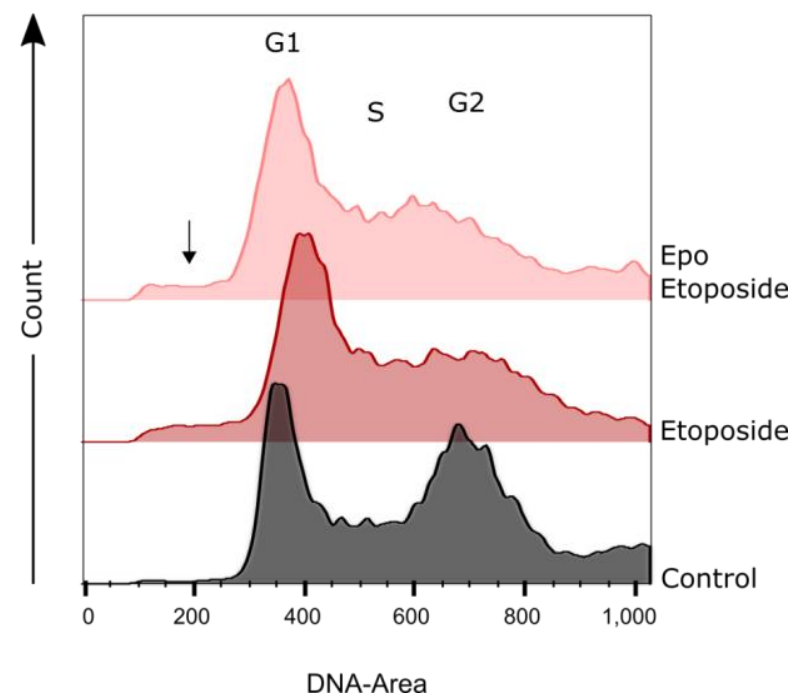

Figure 5-16: Cell viability assessment of HEK293 cells using TUNEL assay and cell cycle analysis. (A, B) Flow cytometric analysis of TUNEL staining with propidium iodide counterstaining. Cells in $\mathbf{A}$ were treated with $20 \mu \mathrm{M}$ etoposide for $26 \mathrm{~h}$ while cells in B are treated in the same way with an additional pre-treatment of $84 \mathrm{ng} / \mathrm{ml}$ Epo for $18 \mathrm{~h}$. Dead cells are depicted in Q2. The pre-treatment with Epo protects against apoptosis (11.2\% vs. $6.4 \%$ ). (C) Exemplary gating of single cells for cell cycle analysis (D) Cell cycle analysis analyzing the propidium iodide fluorescence of the samples shown in $\mathbf{A}$ and $\mathbf{B}$. The G1, S and G2 phases are indicated. Apoptotic cells accumulate in the Sub-G1 population (arrow). Histograms were overlaid with identical scaling. $\mathrm{N}=1$.

These results do not rely on multiple biological replicates but indicate first insights into Epo-induced cell protection in HEK293 cells and demonstrate the feasibility of TUNEL assay and cell cycle analysis. They provide the basis for optimization of etoposide and Epo treatments that will increase the sensitivity of the assay. So far, the apoptosis-inducing impact of etoposide treatment seems too low and has to be increased. If this is optimized, the most effective Epo concentration should be determined in order to detect more pronounced differences in cell viability between etoposide- and etoposide/Epo-treated cells. The herein used concentrations were tested on the basis of previously 
reported studies but seem to need adjustments in future experiments (Eddabra et al., 2012; Hahm et al., 2012; Miller et al., 2015; Sooklert et al., 2016). Furthermore, I suggest to include additional experiments using the neuroprotective splice variant Ev-3 instead of Epo. This would exclude any potential interfering effects resulting from potential EpoR activation although EpoR should not be expressed in HEK293 cells (Heir et al., 2016; Ott et al., 2015). 


\subsubsection{Antibody against Tc-CRLF3}

Although CRLF3 is a sparsely studied cytokine receptor, several commercial antibodies are available. We tested two of them (\#HPA007596, RRID: AB_1847241, Atlas Antibodies, Sweden, and \#sc-398388, Santa Cruz, Germany) in tissues from Tribolium castaneum and Locusta migratoria in immunohistochemical stainings and western blots. They elicited a reproducible staining, however western blot bands were not detected at the excepted size (110 kD instead of $49 \mathrm{kD})$. In addition, there was no immunoreactivity to Drosophila S2 cells heterologously expressing Tc-CRLF3. Hence, their specificity to Lm-CRLF3 was verified by mass spectrometry. Protein lysates of locust hemolymph and brain tissue were separated by sodium dodecyl sulfate polyacrylamide gel electrophoresis (SDSPAGE) into 23 equidistant bands, treated by in-gel digestion, and bands in the range of $110 \mathrm{kD}$ were analyzed by LC/MS/MS for their protein content. However, mass spectrometry did not detect $\mathrm{Lm}$ CRLF3. Hence, we concluded that the commercially available antibodies bind non-specifically to Locusta and Tribolium proteins but do not detect CRLF3 in these species. We thank the Core Facility Proteomics of the University Medical Center Goettingen (Dr. Christof Lenz, Thierry Wasselin) for LC/MS/MS analysis and expert advice.

\subsubsection{Design of a custom-made antibody}

Since we aim to investigate the expression patterns of CRLF3 in the insect brain and to determine the kinetics of soaking RNAi induced knock-down, I designed and ordered a custom-made antibody targeting Tc-CRLF3. The antibody was produced by Proteintech (United Kingdom) applying a 102-daylong immunization protocol including four booster immunizations. The antigen for immunizations spans amino acids 1-300 of Tc-CRLF3 (Figure 5-17).

This region was chosen since the terminus is presumably located extracellularly due to the comprised cytokine receptor motif. Furthermore, the Janus kinase (JAK) docking site, which is positioned on the opposite side of the potential transmembrane region, is assumed to be intracellular. The basis for the antigen production was the vector pUbiP_Tc-crlf3-eGFP. Proteintech performed further processing steps including cloning, protein expression, immunization of two rabbits and affinity purification.

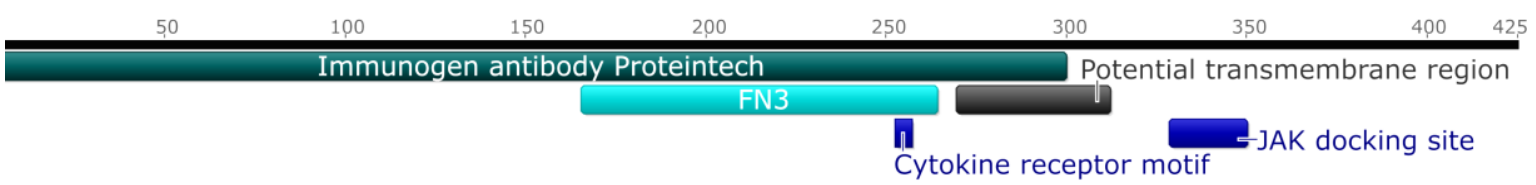

Figure 5-17: Design of the immunogenic peptide of the custom-made antibody targeting Tc-CRLF3 (425 aa). The first 300 aa were used for immunizations of two rabbits. It is likely that this region is located extracellular. FN3 = Fibronectin type-III, JAK = Janus kinase. 


\subsubsection{Validation of the custom-made antibody}

The purified antibodies are currently being tested with the aid of western blots in our lab. The validation procedure comprises the evaluation of different lysates as positive and negative controls and of the pre-immune serum. Results are shown in Figure 5-18. A negative control is a lysate of Drosophila S2 cells. Positive controls are lysates of S2 cells heterologously expressing the fusion protein Tc-CRLF3-eGFP, Tribolium larvae and Tribolium pupae. Western blots were performed on nitrocellulose membranes $(0.45 \mu \mathrm{M})$ and blocked with $4 \%$ milk in PBSTween $0.1 \%$ for 30 min at RT. The primary antibody (rabbit No. 2) was diluted in blocking buffer 1:5000 and incubated over night at $4{ }^{\circ} \mathrm{C}$. The secondary antibody was applied 1:10000 diluted in blocking buffer for $30 \mathrm{~min}$ at RT. The antibody detects no bands in untransfected Drosophila S2 cell serving as negative control (Figure 5-18, Lane 1). It faintly detects proteins with a size of approximately $50 \mathrm{kD}$ in Tribolium pupae and larvae lysates (Lane 3-5). This corresponds to the expected size of Tc-CRLF3 (49 kD). In addition, a prominent band was detected in lysate from S2 cells heterologously expressing Tc-CRLf3-eGFP (Lane 2, $76 \mathrm{kD}$ ). It is the same band that is also detected by the $\alpha$-GFP antibody (not shown). This supports the assumption, that the custom-made antibody originating from rabbit No. 2 specifically detects Tc-CRLF3. For final validation, biological replications will be performed and the pre-immune serum tested.

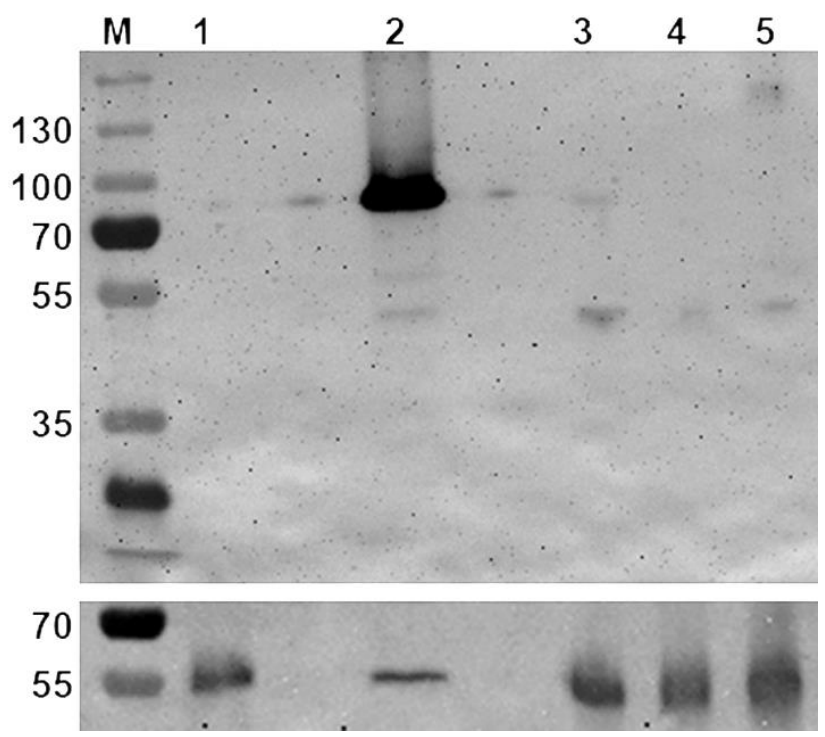

Figure 5-18: Western blot testing the specificity of the custom-made antibody against Tc-CRLF3 from rabbit No. 2. $20 \mu \mathrm{g}$ of protein lysates were loaded. (1) Drosophila S2 cells; (2) Drosophila S2 cell expressing TcCRLF3-eGFP; (3) Tribolium pupae; (4) Tribolium larvae; (5) Tribolium larvae RNAi; (M) Marker in kD. Top: The antibody strongly detects Tc-CRLF3-eGFP in S2 cells (76 kD) and weakly Tc-CRLF3 (49 kD) in Tribolium. Bottom: Loading control alpha-tubulin. Western blot performed by Jannis Hainke. 
If the antibody is validated, it will be used to determine the turn-over rate of Tc-CRLF3 and the success of soaking RNAi-mediated Tc-CRLF3 knock-down. So far, the success of soaking RNAi has been judged on the basis of mRNA levels using qRT-PCR. However, the complementation of data regarding the protein level will strengthen our results and will gain new insights into the expression of CRLF3. Moreover, the antibody will be tested for use in immunohistochemistry. This will offer knowledge about the localization of $T c$-CRLF3, for instance, in different regions of the brain or various tissues outside the nervous system. 


\subsubsection{Drosophila S2 cells heterologously expressing Tc-CRLF3}

Drosophila melanogaster lacks endogenous CRLF3 expression and its cells are not sensitive to recombinant human Epo. Thus, they offer a perfect model system to heterologously express the beetle receptor Tc-CRLF3 and to establish a versatile screening assay for potential ligands and receptor-ligand-interactions. Firstly, I aimed to investigate whether Drosophila S2 cells expressing TcCRLF3 gain Epo sensitivity. To this end, I have established various approaches in our lab that will provide the basis for future experiment using that assay and are summarized in this subchapter. In contrast to the before mentioned assays with Tribolium and locust primary brain cells, this assay using S2 cells has the advantage of a rapid, objective and also reliable determination of cell viability by flow cytometry. Flow cytometry analysis allows automated viability assessment in contrast to manual live/dead cell analysis utilized for Tribolium and Locusta neuronal cultures. Furthermore, the flow cytometer can assess 10.000 cells for its viability within $2-3$ min.

\subsubsection{Culturing of Drosophila Schneider cells}

Drosophila macrophage-like Schneider (S2) cells (\#R69007, Thermo Fisher Scientific, Germany) were cultured in Schneider's Medium (\#21720-024, Thermo Fisher Scientific, Germany) and 10\% FBS Superior (not heat inactivated, \#S 0615, Biochrom, Germany) at $28^{\circ} \mathrm{C}$ in a humidified atmosphere. For general maintenance, they were passaged every 3 - 4 days by mechanical dissociation, centrifugation ( $300 \mathrm{~g}, 5 \mathrm{~min}, \mathrm{RT}$ ) and split 1:5 into new T25 flasks. Cells were used for experiments up to passage P40. Frozen stocks were prepared from healthy cultures with a density of approximately $1 * 10^{7}$ cells $/ \mathrm{ml}$. The cells were harvested, washed in PBS and resuspended in pre-cooled freezing medium ( $45 \%$ conditioned culture medium, $45 \%$ fresh culture medium and $10 \% \mathrm{FBS}$ ). $1 \mathrm{ml}$ of that cell suspension each was aliquoted into cryovials, wrapped in a paper towel, placed into a styrofoam ${ }^{\mathrm{TM}}$ box and stored at $-80^{\circ} \mathrm{C}$. After several days, the cryovials were transferred to a freezer box for long term storage. Thawing of cells was performed quickly in a warm water bath in order to reduce cell damage. When the cells were almost thawed, the cell suspension was immediately diluted in $4 \mathrm{ml}$ of room temperature culture medium (at RT) and spun down ( $300 \mathrm{~g}, 5 \mathrm{~min}, \mathrm{RT}$ ). Finally, the cell pellet was resuspended in fresh culture medium and seeded into a T25 flask.

\subsubsection{Cloning of expression vectors}

In order to heterologously express the beetle receptor Tc-crlf3 in Drosophila S2 cells, they were transfected with the pUbiP_Tc-crlf3-eGFP or pUbiP_Tc-crlf3-eGFP_Puro plasmids (Supplementary Figure 5-4 and Supplementary Figure 5-5). The plasmids were constructed by restriction cloning using cDNA from Tribolium pupae and larvae. The backbone vector pUbiP-c-eGFP (6545 bp, Supplementary Figure 5-2) was kindly provided by Alf Herzig (Molecular Developmental Biology, Prof. Herbert Jaeckle, Max Planck Institute for Biophysical Chemistry, Goettingen, Germany). Its ubiquitin promotor 
drives expression in all tissues and eGFP is a suitable marker to evaluate transfection efficiency and to detect the fusion protein Tc-CRLF3-eGFP. Tc-crlf3 sequences (1272 bp) were cloned into pUbiP using the restriction sites EcoRV and BspOI with the below listed PCR. The reading frame was preserved in order to attach the eGFP tag to TC-CRLF3 (forward primer 5 ' tatgatatct ATGGGTGATAGGAACGAAGTGGTG 3', reverse primer $5^{\prime}$ aatgctagcTTCAACCATTATTTTGGTAG CAGGCGATT 3').

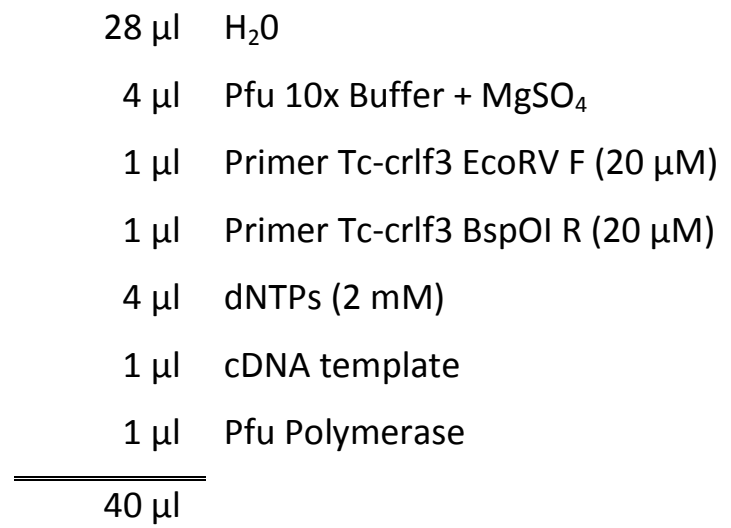

\begin{tabular}{l|l} 
PCR program: & \\
$98{ }^{\circ} \mathrm{C}$ & $3 \mathrm{~min}$ \\
$98{ }^{\circ} \mathrm{C}$ & $30 \mathrm{~s}^{*}$ \\
$55^{\circ} \mathrm{C}$ & $30 \mathrm{~s}^{*}$ \\
$72{ }^{\circ} \mathrm{C}$ & $3 \mathrm{~min} *$ \\
$72{ }^{\circ} \mathrm{C}$ & $8 \mathrm{~min}$ \\
$8^{\circ} \mathrm{C}$ & hold \\
& $* 30$ cycles
\end{tabular}

As initial experiments showed that transfected Drosophila S2 cells have to be selected, a puromycine resistance cassette was additionally inserted using HindIII restriction sites. It was restricted from pCoPuro together with the copia promotor (1215 bp). pCoPuro was a gift from Francis Castellino (Addgene plasmid \# 17533, RRID:Addgene_17533) (Iwaki et al., 2003). For control experiments, a vector pUbiP-c-eGFP_Puro was cloned with the same approach (Supplementary Figure 5-3). The Tccrlf3 sequences of larvae and pupae comprise 5 single nucleotide polymorphisms (SNPs) in comparison to the official sequence au3.g2971.t1 (Tc000209). The SNPs are $231 \mathrm{G}>A$ (K77K), $317 \mathrm{~A}>\mathrm{C}$ Y106S), 366 A $>$ T (S122), 775 T>C (S259P) and 788 G>A (R263K). Since the sequences of both larvae and pupae exhibit the identical SNPs, errors resulting from PCR amplification can be excluded.

\subsubsection{Transfection of $S 2$ cells}

One day before transfection, $\mathrm{S} 2$ cells were seeded in T25 flasks. Optimal transfection efficiency was obtained with seeding of $1 * 10^{6}$ cells (Figure 5-19). The transfection was performed with the Effectene transfection kit (\#301425, Qiagen, Germany) according to the user manual with plasmids purified with the aid of the NucleoBond ${ }^{\circledR}$ Xtra Midi Kit (MACHEREY-NAGEL, Germany). However, the transfection reagent was not removed after a few hours because it did not harm S2 cells during the tested time period of 4 days. First eGFP fluorescence was detected on day 1 but strong fluorescence was present from day 2 onwards. Optimal transfection efficiency regarding the DNA/Effectene ratio and the amount of DNA was determined by western blot analysis. Since no reliable antibody 
detecting Tc-CRLF3 was available, an $\alpha$-GFP antibody was used to detect the fusion protein Tc-CRLF3eGFP.

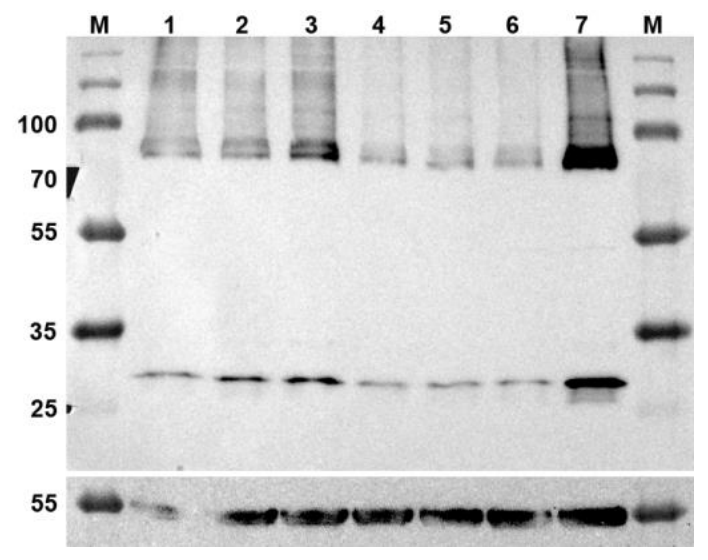

Figure 5-19: Western blot analysis to evaluate the transfection efficiency in Drosophila S2 cells. . Protein lysate in each lane is $75 \mu \mathrm{g}$. (1) $1 \mu \mathrm{g}$ DNA, DNA/Effectene 1:25, 5*10 cells; (2) $1 \mu \mathrm{g}$ DNA, DNA/Effectene 1:50, $5 * 10^{5}$ cells; (3) $2 \mu \mathrm{g}$ DNA, DNA/Effectene $1: 25,5 * 10^{5}$ cells; (4) $2 \mu \mathrm{g}$ DNA, DNA/Effectene 1:50, $5 * 10^{5}$ cells; (5) $1 \mu \mathrm{g}$ DNA, DNA/Effectene 1:25, $1 * 10^{6}$ cells; (6) $1 \mu \mathrm{g}$ DNA, DNA/Effectene 1:50, $1 * 10^{6}$ cells; (7) $2 \mu \mathrm{g}$ DNA, DNA/Effectene 1:25, $1 * 10^{6}$ cells. (M) Marker in $\mathrm{kD}$. Top: The fusion protein Tc-CRLF3-eGFP (76 kD) is detected by an $\alpha$-GFP antibody. Due to a deficient proteinase inhibitor, protein lysates started to degrade resulting in dissociation of eGFP $(27 \mathrm{kD})$. Best transfection efficiency is achieved by $2 \mu \mathrm{g}$ DNA, DNA/Effectene ratio 1:25 and a seeding of $1 * 10^{6}$ cells (lane 7). Bottom: Loading control alpha-tubulin.

TC-CRLF3 has a predicted molecular mass of $49 \mathrm{kD}$ and molecular mass of eGFP is $27 \mathrm{kD}$, thus, the fusion protein is expected at $76 \mathrm{kD}$. In brief, proteins were separated with $10 \%$ SDS PAGE and blotted onto nitrocellulose membranes. They were blocked with $4 \%$ milk solved in PBSTween $0.1 \%$ $(1 \mathrm{~h}, \mathrm{RT})$, incubated with the primary antibody $\alpha$-GFP from rabbit (1:1000 in blocking buffer, over night, $4{ }^{\circ} \mathrm{C}$; A-6455, Invitrogen, Thermo Fisher Scientific, Germany) and the secondary $\alpha$-rabbitperoxidase (1:5000 in blocking buffer, $30 \mathrm{~min}, \mathrm{RT}$; \#A9169, Sigma-Aldrich, Germany). Proteins were detected by chemiluminescence using the SuperSignal ${ }^{\text {TM }}$ West Pico PLUS Chemiluminescent Substrate (\#34580, Thermo Fisher Scientific, Germany). The best transfection efficiency was achieved with a with seeding of $1 * 10^{6}$ cells and a DNA/Effectene ratio of 1:25 using $2 \mu$ g DNA (Figure 5-19).

In order to establish a stable cell line exclusively consisting of Drosophila S2 cells expressing the beetle receptor Tc-CRLF3, the transfected cells were selected. Selection was achieved by addition of $10 \mu \mathrm{g} / \mathrm{ml}$ puromycine (stock $10 \mathrm{mg} / \mathrm{ml}$ ) directly after splitting and high dilution of cells. A low density of cells is favorable since puromycine is a translational inhibitor being most effective in proliferating cells (Yarmolinsky and De La Haba, 1959). After 4 passages, the percentage of Tc-CRLF3-eGFP expressing cells was increased from $54 \%$ to $70 \%$. From these passages, stocks were frozen at $-80^{\circ} \mathrm{C}$ for future experiments. In parallel, the selected cells were subjected to cell sorting (Cell-Sorting facility, Prof. Gerald Wulf and Sabrina Becker, University Medical Center Goettingen, Germany) in order to achieve a stable and pure cell line. Unfortunately, these attempts were not successful. 
Hence, I continued with puromycine selection. Cells transfected with the control plasmid pUbiP_eGFP_Puro were treated in parallel with identical approaches.

\subsubsection{Cell viability staining with SYTOX}

For discrimination of dead and live cells, S2 cells were stained with SYTOX'M Red dead cell stain (\#S34859, Thermo Fisher Scientific, Germany), which exclusively penetrates compromised plasma membranes and binds to nucleic acids. In contrast to PI, SYTOX Red is excited by light of $633 \mathrm{~nm}$ wavelength thereby minimizing cross-talk with other fluorescent dyes excited at $488 \mathrm{~nm}$. For flow cytometry, cells in a confluent T25 flask were harvested, washed three times in PBS (pH 7.4) and resuspended in $500 \mu \mathrm{l}$ annexin binding buffer ( $10 \mathrm{mM} \mathrm{HEPES}, 140 \mathrm{mM} \mathrm{NaCl}, 2.5 \mathrm{mM} \mathrm{CaCl} \cdot 2 \mathrm{H}_{2} \mathrm{O}, \mathrm{pH}$ 7.4). $100 \mu$ l of cell suspension were used for each sample and transferred into a FACS tube (\#352052, Falcon ${ }^{\circledR}$, Corning Life Science, United States of America). Cells were spun down (300 g, 5min, RT), resuspended in $300 \mu \mathrm{l}$ SYTOX Red stain dilution 1:1000 in annexin binding buffer and incubated for 30 min at RT in the dark before flow cytometric measurements. SYTOX Red was excited with the $633 \mathrm{~nm}$ laser and detected with the APC filter. Analysis was performed using the BD LSR II flow cytometer and the BD FACSDiva software (version 6.3.1).

\subsubsection{Cell cycle analysis in $S 2$ cells}

Cell cycle analysis was used as a complementary method to determine the viability of S2 cells. The cell cycle was determined with a flow cytometer measuring the intensity of propidium iodide (PI) labeled DNA. Cells were harvested from a confluent T25 flask, washed twice with PBS (pH 7.4) and resuspended in $500 \mu$ l PBS. For each sample, $100 \mu$ l cell suspension were added dropwise to $5 \mathrm{ml}$ icecold $70 \%$ ethanol while vortexing. For fixation, samples were incubated on ice for $30 \mathrm{~min}$ and the cells were slightly swirled from time to time to prevent cell aggregation. After two washing steps with PBS (850 - $1000 \mathrm{~g}, 5 \mathrm{~min}, \mathrm{RT})$ ), cells were thoroughly resuspended in $50 \mu \mathrm{l}$ of RNAse A solution $(100 \mu \mathrm{g} / \mathrm{ml})$. Subsequently, $400 \mu \mathrm{l}$ of PI $(50 \mu \mathrm{g} / \mathrm{ml})$ per $1 * 10^{6}$ cells were added and incubated for $30-$ $60 \mathrm{~min}$ at $37{ }^{\circ} \mathrm{C}$. Finally, cells were analyzed with a flow cytometer using the $488 \mathrm{~nm}$ laser and the PerCP filter.

\subsubsection{Tc-CRLF3-eGFP expression in S2 cells}

Heterologous expression of the eGFP-tagged beetle receptor Tc-CRLF3 was evaluated by mass spectrometry and microscopy. For mass spectrometry, Tc-CRLF3-eGFP was purified using the GFP Selector (\#N0310, NanoTag Biotechnologies, Germany) (protocol shown in supplementary material 5.6.3.7.1). These agarose beads are crosslinked to high-affinity single-domain antibodies and ensure a high affinity as well as capacity. The purification was validated by a coomassie blue staining. The

purified proteins were separated by gel electrophoresis and visualized by colloidal coomassie staining. Protein bands of interest were excised manually and subjected to in-gel digestion followed 
by mass spectrometric protein identification as described (Ott et al., 2015). Sample preparation, data acquisition by nanoLC-MS, and data analysis were performed in the laboratory of PD Dr. Olaf Jahn (Proteomics Group, Max Planck Institute of Experimental Medicine, Goettingen, Germany). The presence of Tc-CRLF3-eGFP was confirmed with a coverage of $62.95 \%$. Furthermore, the distribution of detected peptides verified the expression of the entire fusion protein consisting of TC-CRLF3 and the eGFP tag (Figure 5-20).

Whether CRLF3 is a membrane bound receptor is still under discussion (Boulay et al., 2003; Yang et al., 2009). The expression pattern of Tc-CRLF3-eGFP was analyzed by means of counterstaining with CellMask $^{\mathrm{TM}}$ Deep Red plasma membrane stain (\#C10046, Thermo Fisher Scientific, Germany). Cells were seeded on concanavalin-coated (Concanavalin A, Sigma-Aldrich) coverslips and allowed to attach for $90 \mathrm{~min}$. Then, cell culture medium was replaced with 1:1000 CellMask Deep Red staining solution diluted in PBS (pH 7.4) and incubated for 15 min at RT in the dark. Fixation was conducted using $4 \%$ paraformaldehyde (PFA) for 20 min at RT. After 3 washing steps in PBS, cells were mounted in DABCO and stored until the next day at $4{ }^{\circ} \mathrm{C}$. Images were acquired with the Leica TCS SP2 confocal microscope.

\begin{tabular}{|c|c|c|c|c|}
\hline 1-MGDRNEVVET & $\underline{\text { LKAAKSYLNH }}$ & LENLNKQLEN & $\underline{\text { AENQICETSS }}$ & ETERNIEVTF- 50 \\
\hline 51-SNLLTNLTEI & $\underline{\text { LLNRKDILIK }}$ & KVQKTRKQSL & $\underline{\text { APLDASRSDI }}$ & ESNIEKTKKL-100 \\
\hline 101 -IKEGESLLSG & SRENLAEFSQ & $\underline{K S S F L G S L P A}$ & IPELKEVPYI & SFHCEPNLES-150 \\
\hline 151-ELLDICSQFG & EVSRIAPVQI & SQTVEKPGAI & LVEWQSVDDE & RCVDIQEFRL-200 \\
\hline 201-QRAFGDVISE & KHLEINFSDC & YVGAETQFLV & KDLRPNQLYS & FRVCCKFEGS-250 \\
\hline $251-S D W S P W S L P Q$ & VTKTSIKPFS & WASNRDFVFA & DDNKIATPVK & DAPSLLLSDG-300 \\
\hline 301 -PQFAVGYSVE & $\underline{\text { FSYLEIDNKC }}$ & STIALIAVKE & AAGIKEIRLG & DHSYFALDST-350 \\
\hline 351-GEIYVDGVKK & ATKLPEAAKG & FKICFMCELI & QDDKVRVSID & TCNKQVTYDW-400 \\
\hline 401 -QISKTDKLYF & GALLKSPATK & IMVEASRDPP & VATMVSKGEE & LFTGVVPILV-450 \\
\hline 451 -ELDGDVNGHK & $\underline{\text { ESVSGEGEGD }}$ & ATYGKLTLKF & ICTTGKLPVP & WPTLVTTLTY-500 \\
\hline $501-$ GVQCESRYPD & HMKQHDFFKS & AMPEGYVQER & TIFEKDDGNY & $\underline{\text { KTRAEVKEEG }}-550$ \\
\hline 551-DTLVNRIELK & GIDEKEDGNI & LGHKLEYNYN & SHNVYIMADK & QKNGIKVNEK-600 \\
\hline 601-IRHNIEDGSV & QLADHYQQNT & PIGDGPVLLP & DNHYLSTQSA & LSKDPNEKRD-650 \\
\hline 651-HMVLLEFVTA & AGITLGMDEL & $Y K-672$ & & \\
\hline
\end{tabular}

Figure 5-20: Mass spectrometric analysis of the fusion protein Tc-CRLF3-eGFP heterologously expressed in Drosophila S2 cells. Underlined amino acids represent the detected peptides. Black letters represent TC-CRLF3, green letters eGFP. Coverage $62.95 \%$. 
Figure 5-21 shows the heterologous expression pattern of Tc-CRLF3-eGFP and eGFP in S2 cells. The green fluorescence patterns of Tc-CRLF3-eGFP expressing cells and eGFP expressing control S2 cells clearly differ. Sole eGFP in control cells seems to accumulate in the nucleus, while the fusion protein TC-CRLF3-eGFP is evenly distributed with some accumulations and does not exhibit any fluorescence within the nucleus. Accumulation of eGFP as such in the nucleus is common and might be due to its small size (Seibel et al., 2007). However, detailed knowledge why eGFP accumulates in the nucleus is still missing. Tc-CRLF3-eGFP and CellMask seem to partially overlap but it is not conclusive. Future experiments might additionally focus on Drosophila BG2-c2 cells, which exhibit a more complex morphology and might facilitate the evaluation of an overlap of eGFP and CellMask. Furthermore, cotransfections with a fluorescently tagged membrane protein might help to finally clarify whether CRLF3 is membrane bound receptor (Han et al., 2011; Lee and Luo, 1999).
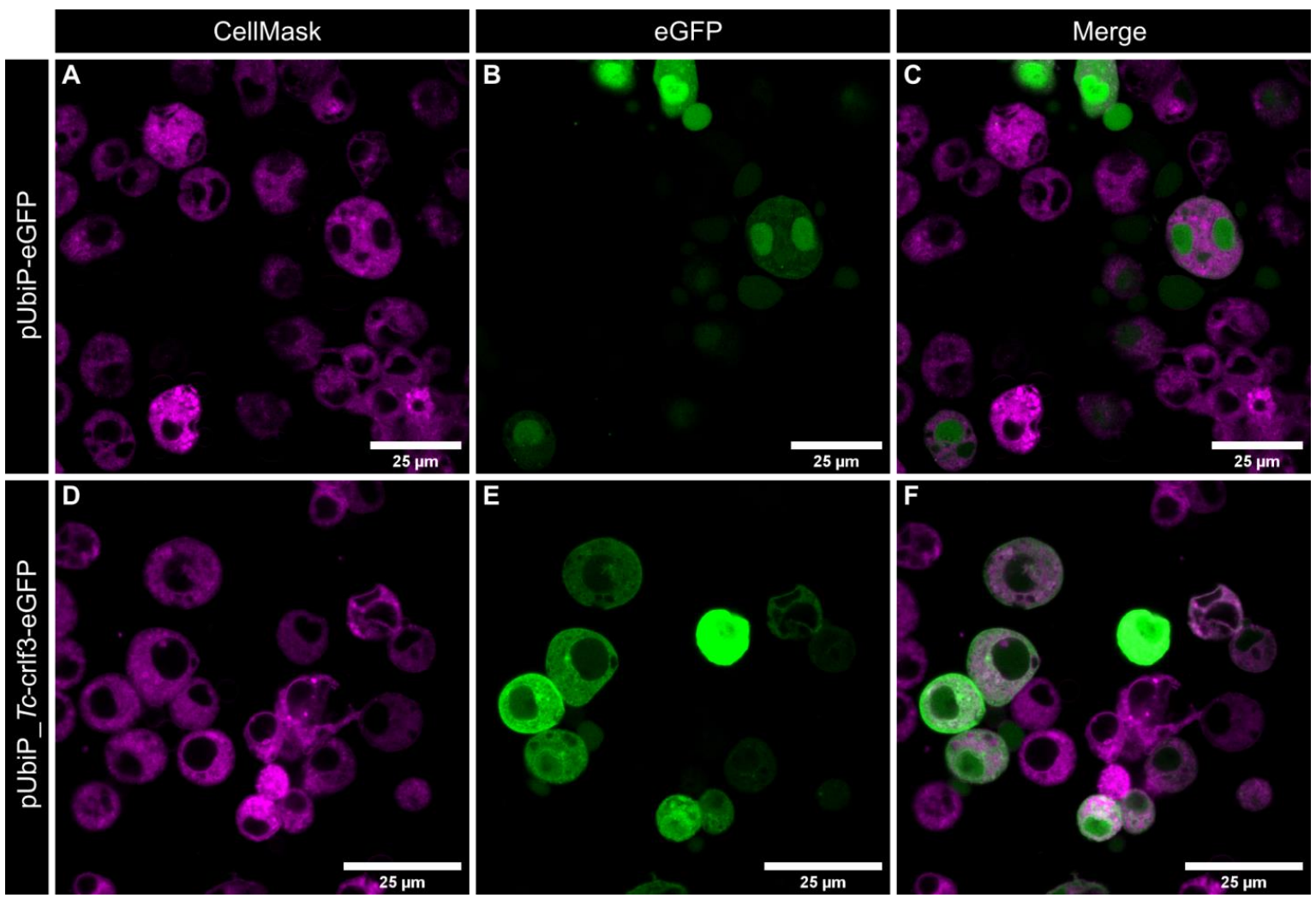

Figure 5-21: Localization of eGFP in transfected Drosophila S2 cells. They were transfected with either pUbiP-eGFP (control, top) or pUbiP_Tc-crlf3-eGFP coding for the eGFP-tagged beetle receptor Tc-CRLF3 (bottom). Cells were counterstained with CellMask Deep Red plasma membrane stain in order to evaluate the localization of the fusion protein Tc-CRLF3-eGFP. While eGFP accumulates in the nucleus, no Tc-CRLF3eGFP proteins are detected therein. Fluorescence signals of Tc-CRLF3-eGFP and CellMask partially overlap. 
A

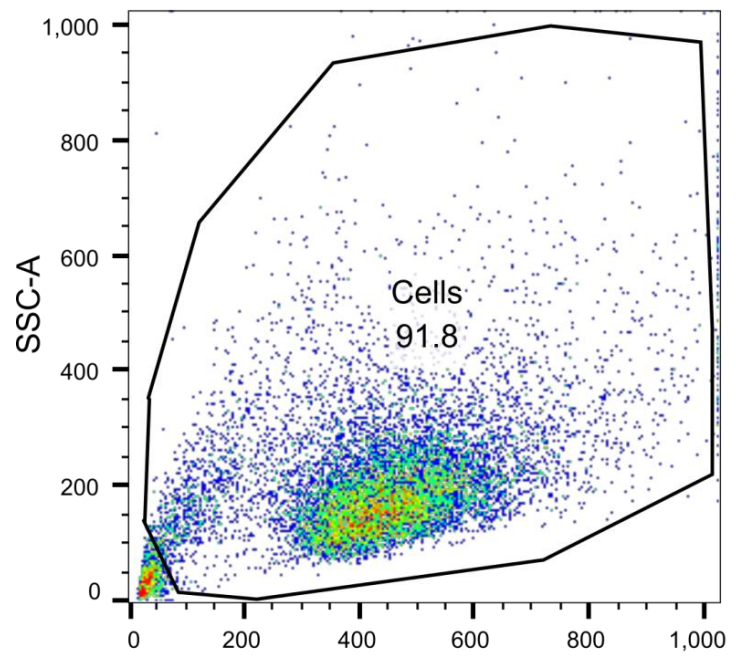

C

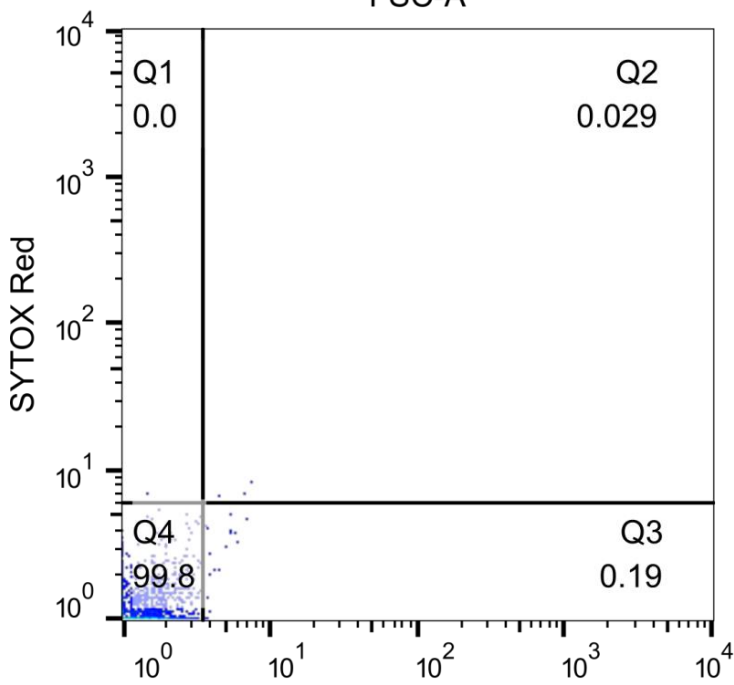

E

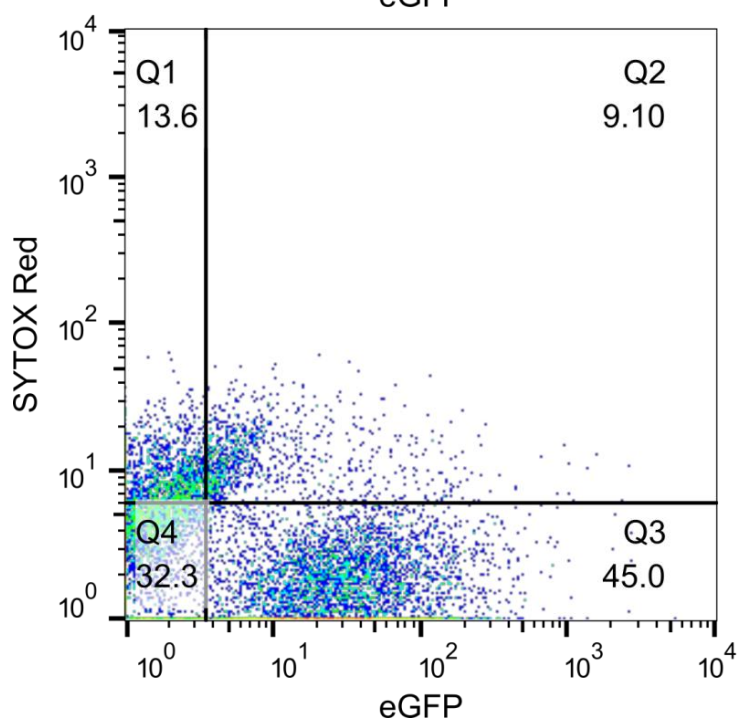

B

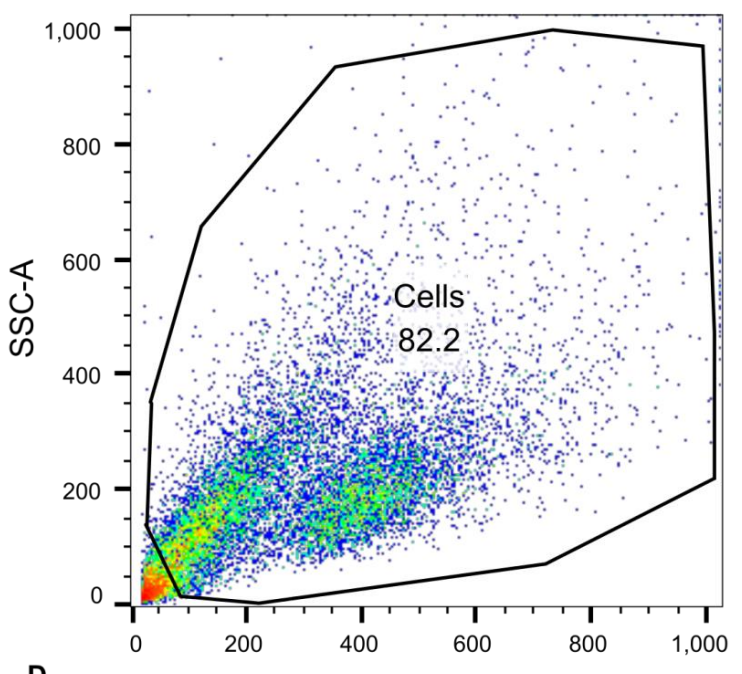

D
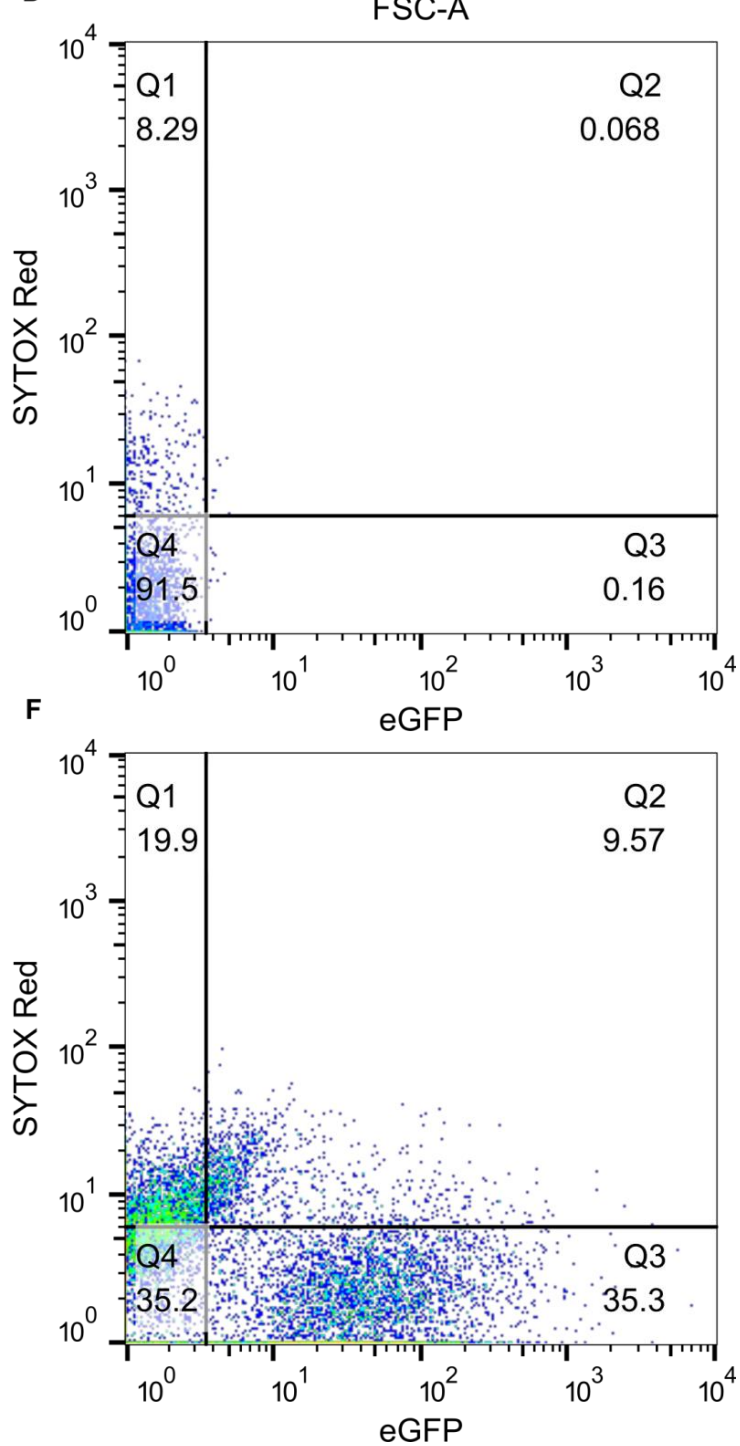

Figure 5-22: Flow cytometric evaluation of Drosophila S2 cells heterologously expressing Tc-CRLF3-eGFP and cell viability. (A) Wild-type non-stained S2 cells. (B) S2 cells heterologously expressing Tc-CRLF3-eGFP. (C) Non-stained wild-type control cells to set eGFP and SYTOX Red gates. (D) SYTOX Red stained wild-type cells under normoxic conditions. (E) SYTOX Red stained TC-CRLF3-eGFP expressing cells under normoxic conditions. (F) SYTOX Red stained Tc-CRLF3-eGFP expressing cells under hypoxic conditions (42 h). 
Drosophila S2 cells expressing TC-CRLF3-eGFP were analyzed using flow cytometry regarding their cell viability and the portion of eGFP positive cells. The gating strategy is depicted in Figure 5-22 A and C with wild-type, non-stained samples. After transfection with pUbiP-Tc-CRLF3-eGFP, $54.1 \%$ of the cells are eGFP positive expressing the beetle receptor Tc-CRLF3 (Q2 + Q3, Figure 5-22 E). In comparison to wild-type S2 cells, they possess an increased population of smaller cells (Figure 5-22 A and B). This population likely represents dead cells. Figure 5-22 C-F shows the evaluation of cell viability by SYTOX Red staining. In wild-type cultures under normoxic conditions $8.37 \%$ of cells were dead (Q1 + Q2, Figure 5-22 D). In contrast, $22.5 \%$ of the cells heterologously expressing Tc-CRLF3eGFP (Q1+Q2 Figure 5-22 E) were dead. If these cells were exposed to $42 \mathrm{~h}$ hypoxia $\left(\mathrm{O}_{2}\right.$ level below $0.4 \%)$, this slightly increased the portion of dead cells to $29.47 \%$. In addition, this resulted in a decrease of eGFP positive cells from $54.1 \%$ to $44.87 \%$ indicating that cells lost their eGFP fluorescence upon dying. This assumption was reinforced by a study on various eGFP-expressing

A

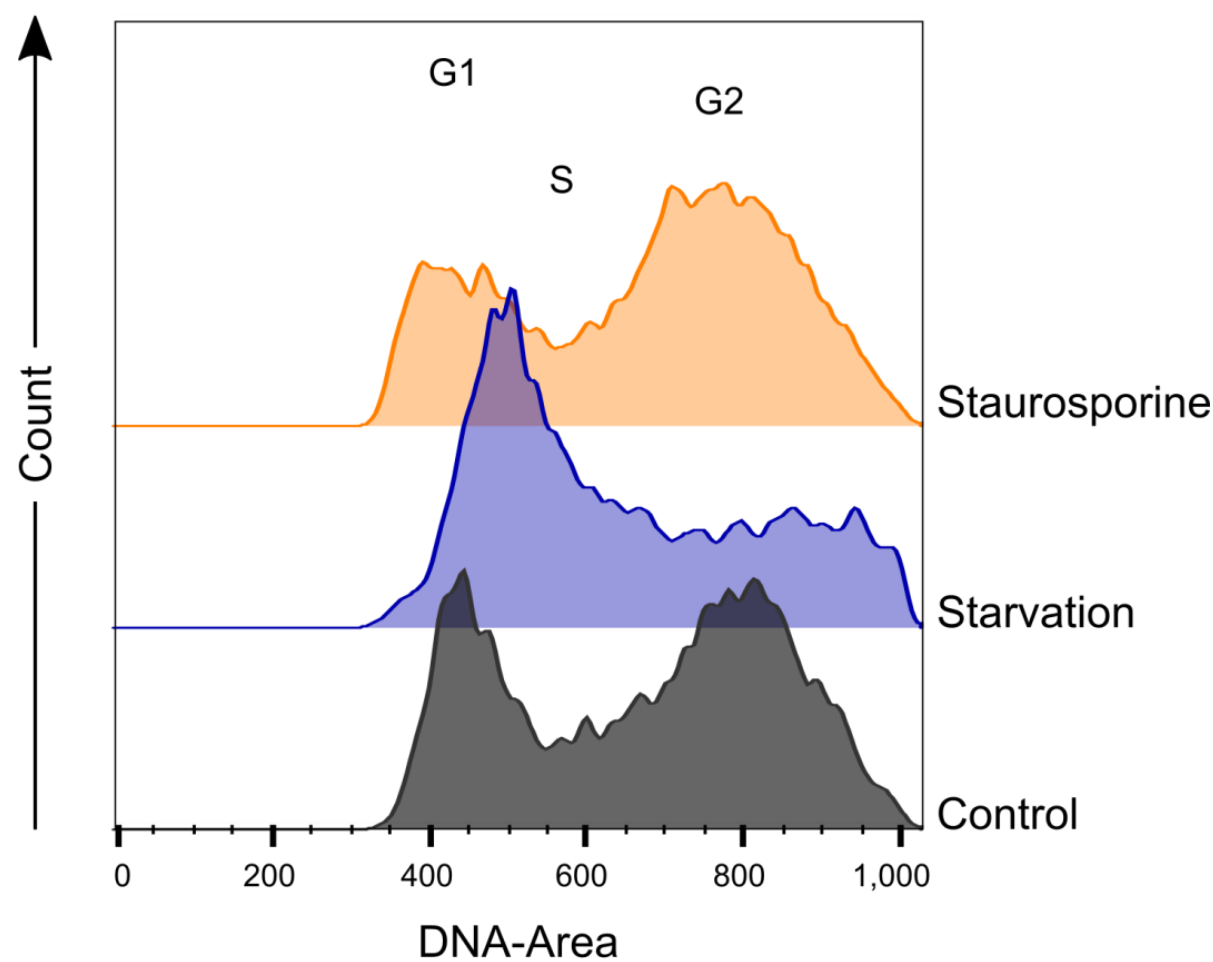

B

\begin{tabular}{lcccc}
\hline & Sub-G1 & G1 & S & G2 \\
\hline Control & $1.82 \%$ & $29.9 \%$ & $14.4 \%$ & $40.0 \%$ \\
\hline Starvation & $1.39 \%$ & $43.7 \%$ & $19.1 \%$ & $20.0 \%$ \\
\hline Staurosporine & $4.35 \%$ & $23.3 \%$ & $15.3 \%$ & $39.9 \%$ \\
\hline
\end{tabular}

Figure 5-23: Cell cycle analysis of staurosporine- and starvation-challenged S2 cells. (A) DNA staining with propidium iodide allowed identification of cell cycle G1, S and G2 phases. Starvation ( $24 \mathrm{~h}$ ) induces cell cycle arrest in $\mathrm{G} 1$ phase. In contrast, staurosporine $(1 \mu \mathrm{M}, 5 \mathrm{~h})$ mildly induced apoptosis, decreased the portion of cells in the G1 phase but slightly increased the amount of cells in $\mathrm{S}$ phase. Histograms with identical scaling were overlaid. (B) Statistics corresponding to the histograms shown in $\mathbf{A} . \mathbf{N}=1$. 
mammalian cell lines showing that eGFP fluorescence but not eGFP protein content decreases after stimulation of apoptosis (Steff et al., 2001). Thus, after hypoxia treatment wild-type and transfected cells are not distinguishable by eGFP fluorescence anymore and wild-type cells cannot serve as an internal control for transfected cells in the same culture. As a consequence, the transfected cells have to be selected by puromycine.

Cell viability assessment using SYTOX Red has been complemented by additional cell cycle analysis using PI (Figure 5-23). Wild-type control cultures exhibited a typical cell cycle consisting of G1, S and G2 phases (29.9\%, $14.4 \%$ and $40.0 \%$ respectively). $24 \mathrm{~h}$ starvation (depletion of FBS) followed by a $24 \mathrm{~h}$ recovery period led to an accumulation of cells in G1 phase (43.7\%) reducing the portion of cells in G2 phase (20.0\%). If cells were treated with the apoptosis inducing agent staurosporine (final concentration $1 \mu \mathrm{M}$ ) for $5 \mathrm{~h}$, a slight increase of apoptotic cells (Sub-G1, $4.35 \%$ ) and slight decrease in G1 phase cells (23.3\%) was observed. Both results match previous studies demonstrating the valid establishment of this method in our lab with our cells. On starvation by FBS depletion, cells stop cell cycle progression due to stress and lack of growth factors. Staurosporine, a topoisomerase I inhibitor, arrests the cell cycle in the $\mathrm{S}$ phase at an early stage (up to $12 \mathrm{~h}$ ) that is later transitioned to an arrest in $\mathrm{G} 2$ phase accompanied by mild induction of apoptosis ( 24 h) (Eng et al., 1988; Jayasooriya et al., 2018).

In conclusion, I herewith provide the basis for future experiments in Drosophila S2 cells that will further investigate the involvement of Tc-CRLF3 in Epo-induced cell protection. For the evaluation of cell viability, the live and dead cell discriminating SYTOX Red stain and cell cycle analysis was established and is now available. Furthermore, these results show that eGFP fluorescence decreases in the course of apoptosis requiring a selection of cells that express Tc-CRLF3-eGFP. Potential methods are cell sorting, the selection with puromycine, cell separation into 96-well plates or a combination of those. Afterwards, selected cells can be tested for a potential gain of Epo sensitivity. If cells expressing Tc-CRLF3-eGFP gain Epo sensitivity, it further substantiates our hypothesis that CRLF3 is a receptor mediating Epo's neuroprotective effect. If not, it does not necessarily disprove our hypothesis. It cannot be ensured that the beetle receptor is functionally integrated into the STAT/JAK signaling pathways in Drosophila S2 cells (presence validated by BLAST search), although we have proven its proper expression by mass spectrometry. If S2 cells heterologously expressing $T c-$ CRLF3-eGFP respond to Epo, they offer an excellent screening assay for interaction studies with various ligands and receptors as well as for the examination of downstream signaling pathways. Amongst others, Tc-CRLF3 receptors with mutations in the WSXWS cytokine motif or JAK docking site might be expressed and screened for signaling functionality. Finally, I suggest expanding these 
experiments to Drosophila neuronal BG2-c2 cells since neuronal cells might react differently in comparison to macrophage-like $\mathrm{S} 2$ cells. 


\subsubsection{Supplementary material}

\subsection{Protocol for $T c$-CRLF3-eGFP purification}

This protocol is adapted from the GFP Selector NanoTag Biotechnologies user manual:

1. Harvest cells and wash twice with PBS.

2. Resuspend cells in $500 \mu \mathrm{l}$ lysis buffer $(150 \mu \mathrm{M} \mathrm{NaCl}, 50 \mathrm{mM}$ TrisHCl, $5 \mathrm{mM} \mathrm{MgCl}, \mathrm{pH}$ 7.5, $1 \%$ Triton-X-100, 1 x Protease inhibitor).

3. Sonicate for $30 \mathrm{~s}$.

4. Freeze at $-20^{\circ} \mathrm{C}$.

5. Thaw and clarify the lysate by centrifugation for $10 \mathrm{~min}, 13.000 \mathrm{~g}$ and $4{ }^{\circ} \mathrm{C}$.

6. Take sample for further analysis (Input fraction).

7. Equilibrate GFP Selector Resin.

a. Resuspend GFP Selector Resin.

b. Transfer $20 \mu \mathrm{l}$ slurry into a clean $1.5 \mathrm{ml}$ reaction tube.

c. Add 1 ml lysis buffer.

d. Centrifuge for $1 \mathrm{~min}$ at $1000 \mathrm{xg}$ and carefully remove supernatant.

e. Repeat steps c-d once.

8. Add clarified lysate from step 2 to equilibrated GFP Selector Resin obtained in step 3.

9. Incubate over night at $4{ }^{\circ} \mathrm{C}$ with head-over-tail rotation.

10. Sediment beads by centrifugation for $1 \mathrm{~min}$ at $1000 \times \mathrm{g}$ and $4{ }^{\circ} \mathrm{C}$.

11. Take sample from supernatant for further analysis (Non-bound fraction).

12. Washing.

a. Carefully remove supernatant.

b. Check if GFP bound to the beads.

c. Resuspend beads in $1 \mathrm{ml}$ lysis buffer. 

d. Centrifuge for $1 \mathrm{~min}$ at $1000 \times \mathrm{g}$.
e. Remove supernatant.

13. Transfer.
a. Remove bottom plug from Mini Spin Column. Place column in $2 \mathrm{ml}$ reaction tube.
b. Resuspend beads in $200 \mu$ l lysis buffer, transfer suspension to Mini Spin column.
c. Wash out beads sticking to tube with $200 \mu$ lysis buffer and transfer to column.
d. Centrifuge column for $1 \mathrm{~min}$ at $1000 \times \mathrm{g}$, discard flow-through.

14. Wash twice with $400 \mu \mathrm{l}$ Washing buffer, centrifuge for $1 \mathrm{~min}$ at $1000 \mathrm{xg}$.

15. Wash once with $400 \mu \mathrm{l} \mathrm{TBS}$, centrifuge for $1 \mathrm{~min}$ at $3000 \times \mathrm{g}$.

16. Attach bottom plug and place Mini Spin Column in a clean $1.5 \mathrm{ml}$ reaction tube.

17. Resuspend GFP Selector resin in $50 \mu \mathrm{l} 2 \mathrm{x}$ Laemmli sample buffer.

18. Heat Mini Spin Column to $95^{\circ} \mathrm{C}$ for $10 \mathrm{~min}$.

19. Remove bottom plug and centrifuge for $1 \mathrm{~min}$ at $3000 \mathrm{xg}$.

20. Analyze the eluate by SDS-PAGE. 


\subsection{Vector map pUbiP-c-eGFP}

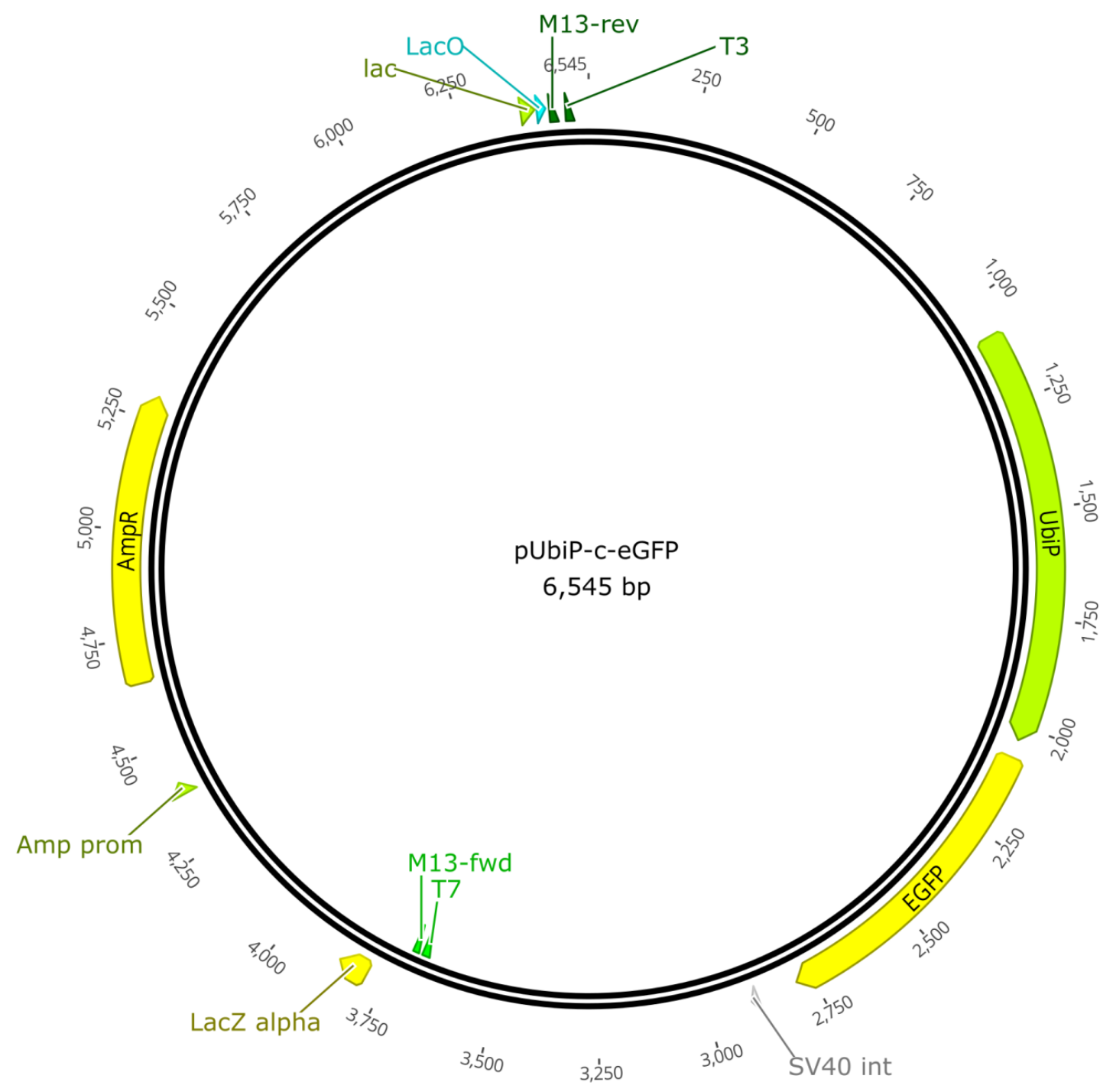

Supplementary Figure 5-2: Vector map of pUbiP-c-eGFP. 


\subsection{Vector map pUbiP-c-eGFP_Puro}

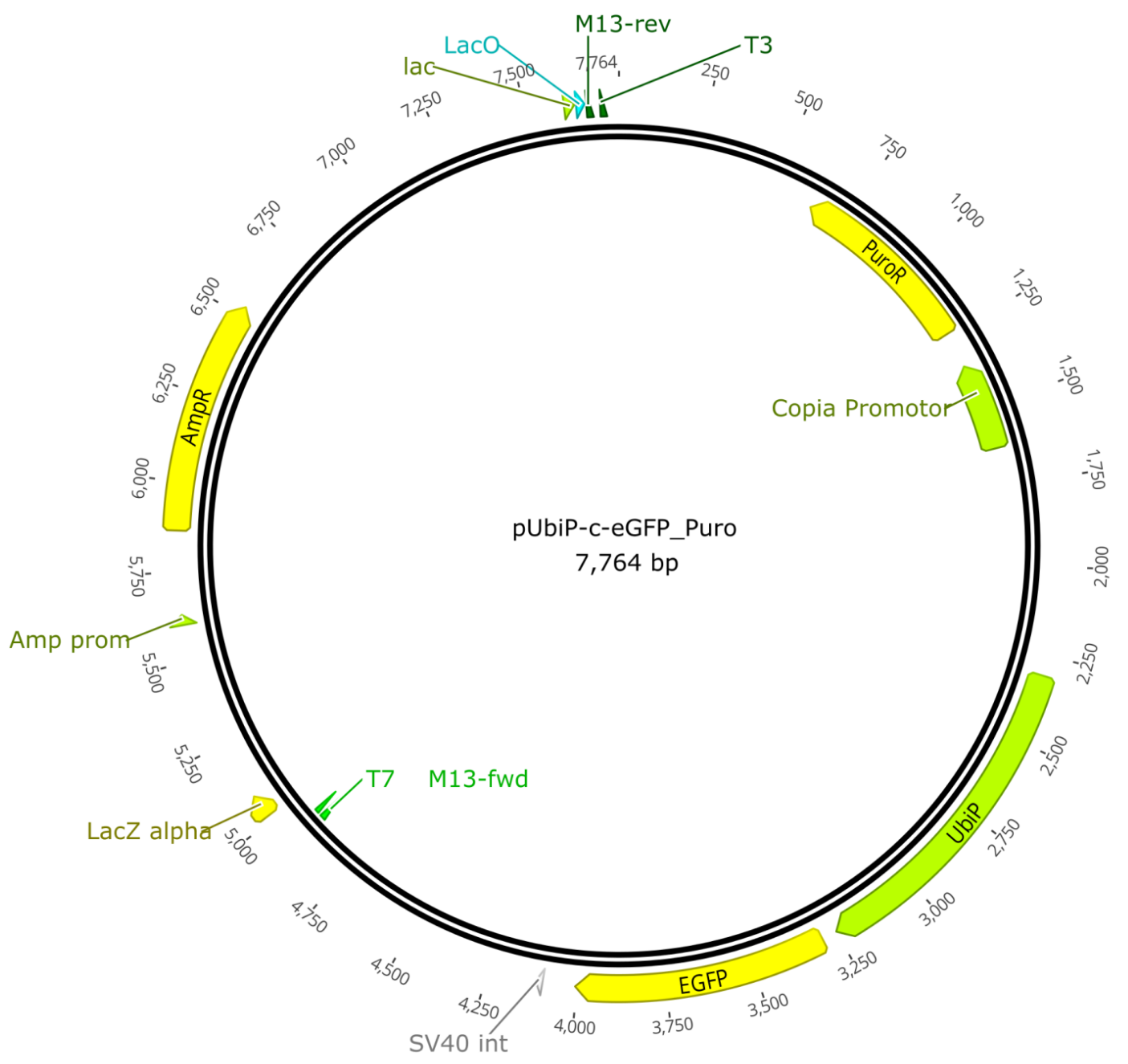

Supplementary Figure 5-3: Vector map of pUbiP-c-eGFP_Puro. 


\subsection{Vector map pUbiP_Tc-crlf3-eGFP}

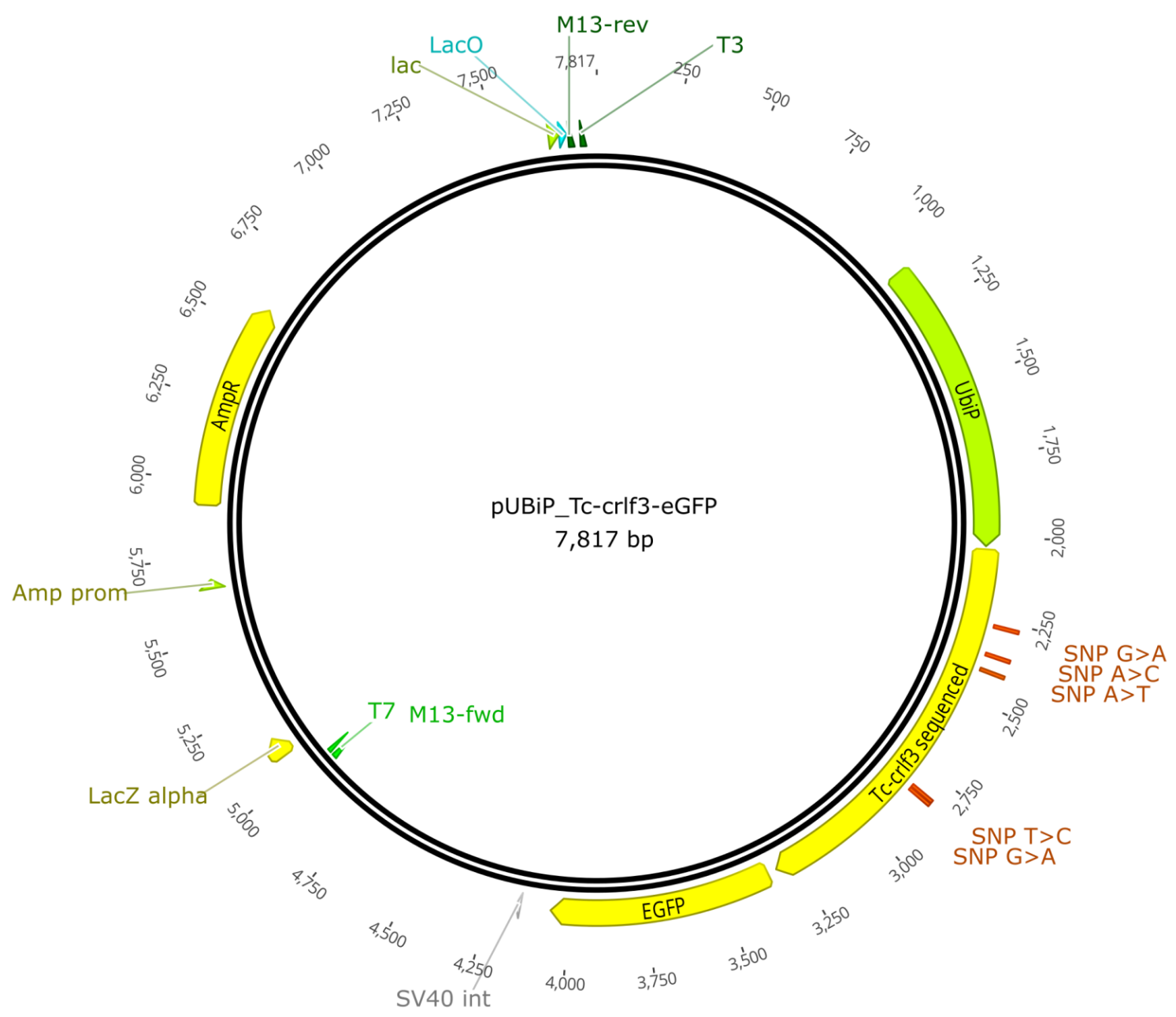

Supplementary Figure 5-4: Vector map of pUbiP_Tc-crlf3-eGFP 


\subsection{Vector map pUbiP_Tc-crlf3-eGFP_Puro}

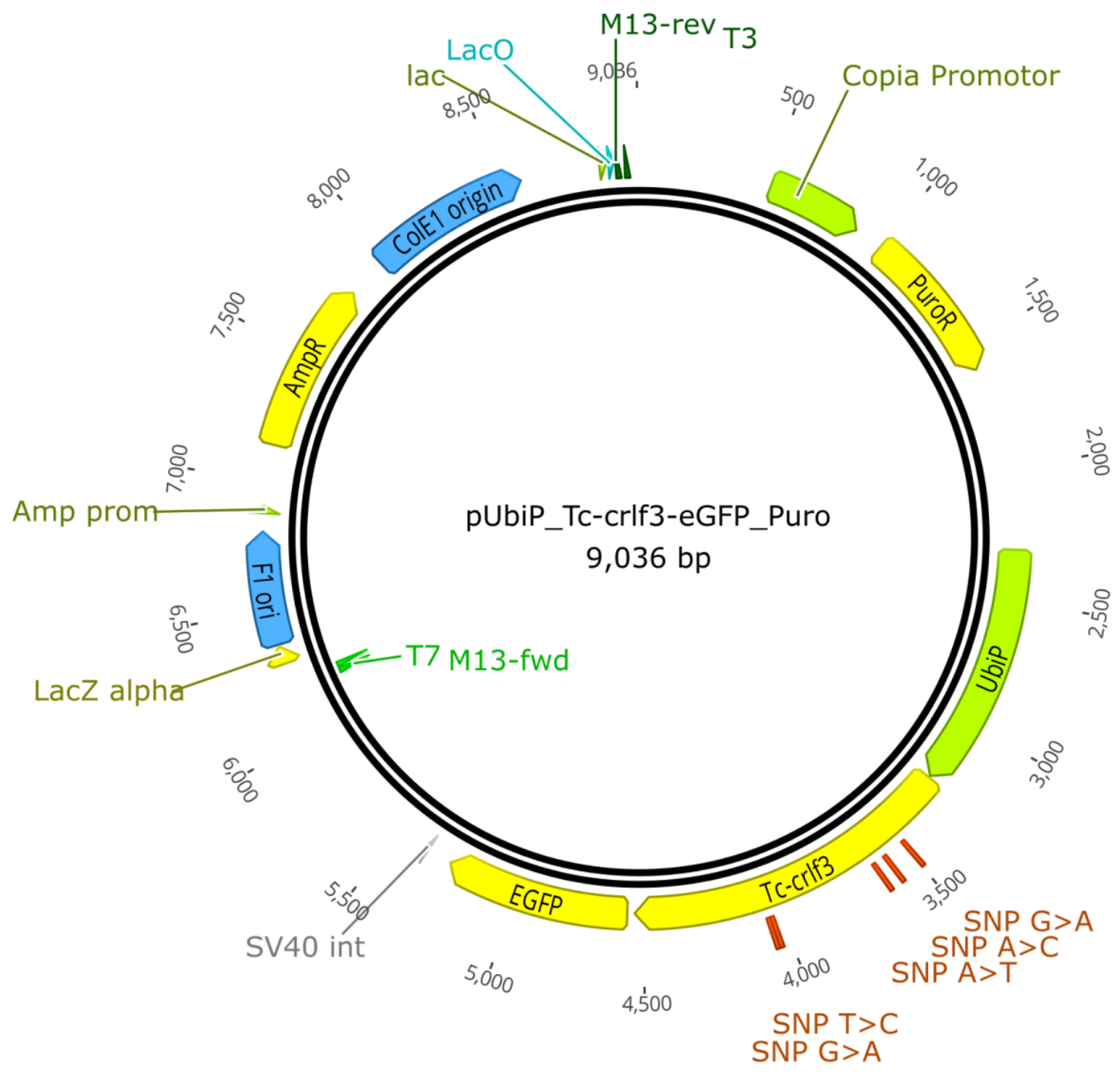

Supplementary Figure 5-5: Vector map of pUbiP_Tc-crlf3-eGFP_Puro 


\section{DiSCUSSION AND OUTLOOK}

There have been only a handful of studies investigating CRLF3, thus, making it a neglected receptor. My work introduced CRLF3 orthologs as neuroprotective Epo-activated receptors. I hereby started to characterize CRLF3 orthologs and demonstrated their importance for future studies. This thesis investigated CRLF3 from a functional and evolutionary perspective. Here, I show the importance of insect CRLF3 in neuroprotection induced by human Epo or Epo-like molecules (Manuscript 1-3) presenting another neuroprotective Epo receptor amongst the $E p o R_{2}$, the $E p o R / \beta c R$ heterodimeric receptor and EphB4 receptor (Brines et al., 2004; Chen et al., 2007; Ostrowski and Heinrich, 2018; Pradeep et al., 2015). Moreover, I demonstrated that locust $L m$-CRLF3 is not only activated by human Epo but also by selective neuroprotective Epo variants and Epo-mimetic peptides (Manuscript 3). My work goes towards identification of signaling motifs that are necessary to activate CRLF3. For instance, HBSP, which consists of 11 amino acids, still induces a neuroprotective effect. This finding proposes a greater similarity of the ligand binding regions between locust and mammalian neuroprotective Epo receptors than between the mammalian neuroprotective and erythropoietic Epo receptors. It further underlines the likelihood that an Epo-like cell protective signaling evolved prior to the vertebrate lineage. In addition, I conducted an in-depth phylogenetic analysis that revealed the simultaneous emergence of CRLF3 with the eumetazoan nervous system (Manuscript 2). The analysis additionally highlights the very high conservation of CRLF3 since the single gene tree of CRLF3 sequences remarkably resembles the eumetazoan tree of life concluded from various conventional markers. Furthermore, the exceptionally high conservation of CRLF3 in vertebrates indicates a fundamental role of CRLF3 for survival. From a more technical point of view, I established Tribolium primary brain cell cultures as a new in vitro model system to study neuronal functions and neuropharmacological characteristics (Manuscript 1). I further improved this model system for loss of function studies by introducing soaking RNAi as a convenient method to induce a gene specific knock-down (Manuscript 1). Additionally, I showed that this method is not only applicable in Tribolium but also in locust primary brain neurons (Manuscript 2) and might be even suitable for other locust cell types (hemocytes are currently tested) or cells from other insect species. Finally, I started to investigate crlf3 expression in different Tribolium tissues and human cell lines (subchapter 5.4 and 5.5).

Since I started the work on CRLF3 from scratch, there remain open questions and many potential follow-up studies. At first, I will mention some direct follow-up studies for which I have already provided the basis by establishing the tools described in subchapter 5.6. Then, I will exemplary describe some perspective follow-up studies that address broader open questions. 


\subsection{Direct follow-up studies}

First of all, future studies should extend my work on crlf3 expression with protein level data for a comprehensive understanding of CRLF3 expression and regulation. To this end, I established a custom-made antibody targeting Tc-CRLF3 (subchapter 5.6.2) whose specificity has been proven by western blot analysis. This antibody could be used to determine persisting protein levels after soaking RNAi-induced knock-down in vitro and the localization of Tc-CRLF3 in whole mount Tribolium brains at different developmental stages. For the evaluation of human Hs-CRLF3 protein levels several CRLF3 antibodies are commercially available and should be tested.

Another point for direct continuation would be characterizing the model system of Drosophila S2 cells heterologously expressing the Tribolium receptor Tc-CRLF3. To test the functionality of the $T c$ CRLF3 receptor, cells should be exposed to hypoxia after being treated with various concentrations of Epo similar to the experiments performed with Tribolium (Manuscript 1) and locust (Manuscript 2) primary brain cells. However, the cell viability of the $\mathrm{S} 2$ cells can be assessed using a flow cytometer which is much faster than cell viability assessment in primary brain cell cultures. I have already established two methods to test S2 cell viability. The first method is based on the cell viability stain SYTOX that exclusively penetrates compromised membranes and stains DNA (subchapter 5.6.3.4). These results can be further validated by additional cell cycle analysis to evaluate the apoptotic SubG1 population (subchapter 5.6.3.5). In locust and Tribolium, I have shown that Epo-induced neuroprotection is dependent on CRLF3 expression by knocking down this receptor. For S2 cells, I have prepared a plasmid solely expressing the eGFP-tag as a control instead. Since S2 cells heterologously expressing $T c$-CRLF3 are more customizable and more convenient than primary brain cells, they build the foundation for further studies. This model system will allow the rapid screening for potential Tc-CRLF3 ligands or the testing of new Epo variants (e.g. Epo derivatives or different glycosylation patterns) as a proof of principle. While S2 cells are suited for the examination of Epoinduced general cell protection, Drosophila neuronal BG2-c2 cells will be eligible for the investigation of Epo's neuroprotective effect.

In order to further test the relevance of Hs-CRLF3 for clinical therapies, its involvement in Epoinduced cell and neuroprotection should be examined in human cells. HEK293 cells are a common model system and are suitable for the investigation of cell protection while the neuroblastoma cell line SH-SY5Y will be available to study neuroprotection. Similar to the insect studies, human cells should be first tested for Epo's cell protective effect under stressful conditions (hypoxia or etoposide). Then, Hs-CRLF3 should be knocked down to evaluate its involvement in Epo-stimulated signaling pathways. The results might be complemented by additional overexpression of Hs-CRLF3 which is technically not feasible in primary insect cells. In human cells, soaking RNAi will presumably 
not be applicable. Instead, knock-down in human cells could be achieved by viral transduction using lentiviruses. This approach ensures the integration of short hairpin RNA (shRNA) into the host genome which results in a cell line stably expressing shRNA inducing a target specific knock-down. Since lentiviruses are assigned to biosafety level 2 , it is not possible to conduct these types of experiments in our research laboratory. Hence, we collaborate with the Gruber Lab (PD Dr. Jens Gruber, German Primate Center, Goettingen, Germany). Nevertheless, I have already established the following initial steps and optimized necessary tools concerning this project. I have tested CRLF3 expression in HEK293 cells (subchapter 5.5.3.1) and non-differentiated as well as differentiated SHSY5Y cells (subchapter 5.5.3.2). Furthermore, I performed Epo and etoposide treatments of HEK293 cells in order to characterize their endogenous Epo sensitivity. To evaluate the cell viability, I established the TUNEL assay and cell cycle analysis in our lab (subchapter 5.6.1). Finally, I cloned the pLKO.1_shRNA_CLRF3 vector carrying the shRNA sequence for viral transduction (Moffat et al., 2006). 


\subsection{Perspective follow-up studies}

\subsubsection{Investigating the downstream signaling pathways of Tc-CRLF3 using RNA sequencing}

In this thesis, I have shown that the insect orthologs Tc-CRLF3 and Lm-CRLF3 are crucial components of Epo-induced neuroprotection. However, exact regulation of the neuroprotective signaling pathways remains enigmatic. It is essential to understand these downstream signaling pathways in order to assess the risks and side effects of potential therapeutic strategies using Epo or Epo variants stimulating the CRLF3 receptor. Pursuing this goal, it will be important to investigate the signaling pathways that play a role in Epo-induced neuroprotection and are regulated by CRLF3 activation. To this end, comparative RNA sequencing (RNA seq) would be an unbiased and sensitive approach uncovering the signaling pathways regulated by CRLF3 in primary insect brain cells. Differentially expressed genes (DEGs) could be determined by comparing the transcriptome upon hypoxia exposure with additional Epo treatment of control neurons and identically treated neurons that suffer from a CRLF3 knock-down. The additional Epo-treatment would ensure the stimulation of CRLF3 dependent neuroprotective signaling pathways. Both Locusta and Tribolium would be appropriate model systems for this study since they both are amenable for soaking RNAi and express CRLF3 orthologs, which are involved in Epo-induced neuroprotection. However, Tribolium is preferable with regard to bioinformatic resources and availability of established experimental protocols.

To the present, CRLF3 signaling pathways have only been investigated in HEK293T cells (Yang et al., 2009). Overexpression of $H s$-CRLF3 induced cell cycle arrest in G0/G1 phase by preventing the entry into the S phase. Knock-down of Hs-CRLF3 led to a reduction of the cell population in the GO/G1 phase but to an increased portion of cells in the $S$ phase. In order to investigate this effect in more detail, the authors of that study used western blot analysis to evaluate components of common signaling pathways. They demonstrated that CRLF3 significantly upregulates cyclin D1 and cyclin D3 while exhibiting slight upregulation of p27 (Yang et al., 2009). Cyclin D1 mediates resistance to apoptosis in various cell types including neurons (Kranenburg et al., 1996; Roué et al., 2008). Cyclin D3 coordinates the cell cycle as well and is upregulated in Parkinson diseased lymphoblasts (Alquézar et al., 2015; Sankaran et al., 2012). In addition, upregulation of p27 causes cell cycle arrest at G0/G1 phase (Kathrein et al., 2005; Liu et al., 2008). The upregulation of Cyclin D1 by CRLF3 is mediated by the STAT3 pathway and is independent of ERK which indicates an independence of the RAS/MAPK pathway (Yang et al., 2009). However, Yang et al. suggest further experiments to examine these interdependencies since these results contradict other studies in which cyclin D1 usually acts on the early to middle G1 phase (Fu et al., 2004). The study I am proposing aims to close this 
scientific gap elucidating the specific neuroprotective signaling pathways downstream of CRLF3 activation using comparative RNA seq. In addition, the experiments suggested would consider the question of CRLF3 being abundantly expressed in various tissues while causing apparent cell cycle arrest.

Appropriate controls are critical for comparative RNA seq analysis. Since RNA seq is expensive, it is very important to include only necessary control groups. Table 6-1 lists all potential experimental groups that would serve as a control for the applied parameters "Normoxia", "Hypoxia", "Epotreatment" and "RNAi treatment". The parameter "RNAi treatment" would be controlled by the application of dsRNA targeting the transcript of dsRed, a protein that is not endogenously expressed in Tribolium, and hence should have no effect on gene expression downstream to Tc-CRLF3. In an initial experiment, I would evaluate the impact of these parameters on the expression of Tc-crlf3 and Tc-stat3 (Tribolium stat3 orthologue TC013218, (Dönitz et al., 2015)) by qRT-PCR. The expression of Tc-stat3 is of interest since STAT3 is activated downstream to CRLF3 (Yang et al., 2009). These results would determine the final experimental conditions that would be included in the RNA seq analysis. If Epo-treatment as such, hypoxia and the application of dsRNA itself do not alter Tc-crlf3 and Tc-stat3 expression, the five experimental conditions highlighted red in Table 6-1 would be sufficient. The main experimental condition would be "Hypoxia + Epo".

Table 6-1: Draft of experimental conditions for RNA seq including all potential control groups. Red highlighted treatment groups might present the final experimental conditions. wt wild-type.

\begin{tabular}{|llll|}
\hline Normoxia & Hypoxia & Hypoxia + Epo & Normoxia + Epo \\
\hline wt & wt & wt & wt \\
\hline RNAi Tc-crlf3 & RNAi Tc-crlf3 & RNAi Tc-crlf3 & RNAi Tc-crlf3 \\
\hline RNAi dsRed & RNAi dsRed & RNAi dsRed & RNAi dsRed \\
\hline
\end{tabular}

For RNA seq analysis, up-to-date transcriptome data would be required that is ensured by the dedicated and active Tribolium community which frequently generates updates of genome and transcriptome resources. Mapping of the raw reads against the transcriptome could be performed using the Bowtie 2 tool (Langmead and Salzberg, 2012). Subsequently, the reads could be counted with the aid of SAM tools. Differential expression calling could be performed with DESeq2 and edgeR packages and top candidates of DEGs could be selected by log2 fold change (Love et al., 2014; McCarthy et al., 2012; Robinson et al., 2009). The expression of these candidates should be assessed by qRT-PCR in order to validate the RNA seq results. Furthermore, the candidates could be compared to RNAi phenotypes described in the iBeetle base (Dönitz et al., 2015). This database already summarized many gene functions in Tribolium. 
In order to evaluate the functional relevance of the identified DEGs, they could be assigned to gene ontology (GO) terms. Respective tools are available for Tribolium including Blast2GO (Götz et al., 2008) and WEGO (Ye et al., 2006). GO term enrichment in biological processes would be of major interest. Moreover, DEGs should be subjected to KEGG (Kyoto Encyclopedia of Genes and Genomes) (Kanehisa and Goto, 2000) pathway mapping which is part of the KEGG PATHWAY data base. This tool would be of main interest because it focuses on molecular interaction networks. Presumably, this would substantially help finding candidates of the neuroprotective signaling pathways downstream to Tc-CLRF3 activation by Epo. Final evaluation of GO term and KEGG enrichment would lead to selection of candidates in Tc-CRLF3 regulated neuroprotective signaling pathways. These candidates could then be evaluated in detail in future studies using soaking RNAi in Tribolium primary brain cells and genome editing in human neuronal cell lines (Kramer et al., 2018; Torres-Ruiz et al., 2016).

\subsubsection{Determination of Tc-CRLF3 signaling and binding motifs}

As I established the artificial expression of Tc-CRLF3 in Drosophila cells, it offers the advantage to particularly modify the receptor in order to identify binding and signaling motifs. For instance, the prototypic cytokine binding motif WSXWS of class I cytokine receptor could be omitted. If this eliminates the sensitivity to Epo, the WSXWS motif is crucial for the Epo-induced Tc-CRLF3 signaling. Subsequently, point mutations could be introduced by site-directed mutagenesis to investigate each amino acid separately. Furthermore, larger portions of TC-CRLF3 could be omitted to narrow down sequence segments that are important for Tc-CRLF3 signaling. Potential targets could be a JAK docking site, the potential transmembrane domain or the ligand binding site. Class I group 1 cytokine receptors as CRLF3 predominantly form homodimers but some heterodimers have also been described (Brines et al., 2004; Langenheim and Chen, 2009; Liongue and Ward, 2007). It is not known whether and how CRLF3 forms homodimers. This could also be examined by stepwise modifying the receptor narrowing down the potential interaction site for homodimerization. Additionally, potential dimerization partners of CRLF3 could be co-expressed with Tc-CRLF3.

Co-immunoprecipitation to identify potential neuroprotective Epo receptors was mostly not successful due to the low cell surface expression of these receptors (Becker et al., 2010). In line with this, co-immunoprecipitation focusing on potential dimerization partners also failed. The proposed approach circumvents this issue because the heterologous expression of Tc-CRLF3 yields high receptor abundance due to the use of the ubiquitin promotor (compare subchapter 5.6.3.6). On the one hand, this system offers the possibility of co-immunoprecipitation studies investigating whether Epo directly binds to TC-CRLF3 or activates it indirectly. On the other hand, it enables co- 
immunoprecipitation to screen for the endogenous ligand of CRLF3 through incubation with various homogenates (compare subchapter 6.2.3).

\subsubsection{Potential approaches to find the endogenous ligand of CRLF3}

Another important follow-up study is the identification of the endogenous ligand of CRLF3. In insects, the endogenous ligand has to be different from Epo since insects do not express Epo (Ostrowski et al., 2011). In vertebrates, CRLF3 might be stimulated by endogenous Epo. I hypothesize that there is at least one other ligand than Epo in vertebrates and that there are orthologues of this ligand in insects as well. This assumption is based on the high phylogenetic conservation and the prebilaterian emergence of CRLF3 (compare Manuscript 2). Hence, identifying the endogenous ligand of Tc-CRLF3 or Lm-CRLF3 might be important for the understanding of vertebrate CRLF3 signaling as well.

Many approaches for ligand identification are possible. As I have shown before (Manuscript 3), various Epo-mimetic peptides are neuroprotective by stimulating $L m$-CRLF3. Their size ranges from 11 to 20 amino acids showing that as few as 11 amino acids are sufficient to induce neuroprotection. One possibility is to consecutively shorten that sequence to find the minimal necessary number of amino acids. Amino acids should be omitted arbitrarily starting from both ends of the peptide in order to test various combinations of peptides. This will mainly provide insights if the binding motif depends on the primary amino acid sequence. Another bioinformatics approach is to find the sequence or spatial structure that all of the tested neuroprotective peptides have in common. To this end, it would be necessary to predict the spatial geometry of all peptides to find commonalities in their three-dimensional structure.

It might be that these synthetic Epo mimetics are neuroprotective but do not directly bind to $\mathrm{Lm}$ CRLF3. Then, unbiased experimental approaches are required to screen for unknown ligands. As mentioned in manuscript 2 , the endogenous ligand is most likely present in the hemolymph. Locust hemolymph should be tested for neuro- and general cell protective effects. If the hemolymph acts in a protective manner, the ligand could be narrowed down by size exclusion. Apart from that the ligand could be crosslinked to Tc-CRLF3 heterologously expressed in S2 cells for coimmunoprecipitation. A specific antibody is presented in this study (subchapter 5.6.2). In addition, the results could be validated with an anti-eGFP antibody since Tc-CRLF3 is tagged with eGFP (subchapter 5.6.3.6). Following to co-immunoprecipitation, the complexes could be analyzed by mass spectrometry. In addition to hemolymph, brain lysates from locusts or mice could be used to separate and identify the endogenous CRLF3 ligand. It might be advantageous to use lysates that are derived from hypoxia-challenged tissues given that this might enhance the amount of the 
endogenous, neuroprotective ligand. It has been shown in a previous study that locusts express an Epo-like molecule (approximately $38 \mathrm{kD}$ ) in the central nervous system that is detected by an antiEpo antibody. Interestingly, this antibody detects further Epo-like molecules at a similar size in other invertebrate central nervous systems but not in Drosophila (Ostrowski et al., 2011). This is in line with the lack of a CRLF3 ortholog and Epo sensitivity in Drosophila. Hence it will be worth to investigate this Epo-like molecule in Locusta by SDS PAGE and mass spectrometry as well. 


\section{ACKNOWLEDGMENTS}

Firstly, I would like to thank Prof. Ralf Heinrich and Prof. Gregor Bucher for starting this project together and thereby offering me to join this fascinating topic. Thanks as well for being in my thesis committee and for your advice in all its facets.

Furthermore, I would like to thank all members of my examination board and Prof. Martin Göpfert for his generous financial support.

Many thanks to Margret Winkler, Stephanie Pauls, Silvia Gubert, Tatjana von Openkowski and Gudrun Matthes for keeping the lab and the office running.

Special thanks to Nicola Schwedhelm-Domeyer who taught me a lot of molecular stuff and many tricks to do lab work in the most efficient way. Thanks for sharing your helpful experience with me and for all the willingness to discuss and optimize project plans. A technician like you is priceless! In addition, I would like to acknowledge Hanna Pies who continued Nicolas work during maternity leave. You learned very fast, did a great job as well and supported me a lot.

I would like to emphasize Debbie Knorr who supported me substatially. It started with optimizing cell culture lab organization, was continued with helpful scientific (and non-scientific) discussions or chocolate supply and ended up with tons of proofreading. Thank you very much, DYK!

I would like to thank Dr. Natascha Zhang for being some kind of a mentor, sharing your impressive molecular biological knowledge with me and providing advice even after you left the lab.

Thanks to Dr. Krissy Corthals and Dr. Robert Kossen for sharing PhD life and providing feedback. I am looking forward to meeting you again, maybe at a neuroscience conference.

I would like to acknowledge the support by various Bachelor's and Master's students emphasizing Johannes, Luca, Sarah, Pia and Henning.

I acknowledge all members of the Göpfert lab who provided plenty of advice, diversified my daily routine, told anecdotes and brought cake. It was a great time with you.

I want to thank my collaboration partners who provided a diverse research environment. Thanks to Rafael Rinaldi and Dr. Jens Gruber for assisting me with studies regarding human CRLF3. Thanks to Prof. Hannelore Ehrenreich, Dr. Olaf Jahn and Dr. Liane Wüstefeld for support and trust within the Epo-mimetic peptide study. 
Thank you, Dr. Sven Bradler, for teaching me good scientific practice, for sharing behind the scene impressions in science and for giving Sarah the opportunity to join CRLF3 phylogeny studies. Sarah Bank-Aubin, it was a pleasure to work with you on CRLF3 phylogeny. Thanks for your advice and introducing me into the fascinating world of phylogeny!

I would like to express my gratitude to Elke Küster and Claudia Hinners from the Bucher Lab for the warm welcome and support although I was from a "foreign" lab.

I would like to acknowledge Dr. Marita Büscher. We started with a controversy and ended up in a friendly and interesting collaboration. If I needed profound advice regarding qPCR, brain morphology or molecular biology, you shared your experience with me.

I would like to thank my former master degree course fellows for supporting each other even during PhD studies.

To all the others I forgot here - I am sorry. I hope that I told you "Thank you" while we were working together.

Thanks to my friends who are not working in science and often had to accept that I was very busy but nevertheless accompanied me. Sometimes, you were the guys keeping me sane.

I greatly acknowledge the support of my family. Thank you for always backing me up, for teaching me to be curious and telling me that comprehension is more important than good grades. I took your advices seriously and will continue.

Last but not least, I would like to thank Steffen for challenging me with tons of questions from an engineering point of view. Thanks for not only being my fantastic husband but also my best buddy, a dedicated supporter and the person who kept me motivated. I greatly acknowledge that you always stepped in when others failed and patiently prepared me for various challenges. 


\section{BibLIOGRAPHY}

Ahmet, I., Tae, H. J., Brines, M., Cerami, A., Lakatta, E. G., and Talan, M. I. (2013). Chronic administration of small nonerythropoietic peptide sequence of erythropoietin effectively ameliorates the progression of postmyocardial infarction-dilated cardiomyopathys. $J$. Pharmacol. Exp. Ther. 345, 446-456.

Ahmet, I., Tae, H. J., Juhaszova, M., Riordon, D. R., Boheler, K. R., Sollott, S. J., et al. (2011). A small nonerythropoietic helix B surface peptide based upon erythropoietin structure is cardioprotective against ischemic myocardial damage. Mol. Med. 17, 194-200. doi:10.2119/molmed.2010.00235.

Aigouy, B., and Mirouse, V. (2013). ScientiFig: A tool to build publication-ready scientific figures. Nat. Methods 10, 1048. doi:10.1038/nmeth.2692.

Alquézar, C., Barrio, E., Esteras, N., De La Encarnaciõn, A., Bartolomé, F., Molina, J. A., et al. (2015). Targeting cyclin D3/CDK6 activity for treatment of Parkinson's disease. J. Neurochem. 133, 886897.

Anderson, P., and Kedersha, N. (2006). RNA granules. J. Cell Biol. 172, 803-808.

Arcasoy, M. O. (2008). The non-haematopoietic biological effects of erythropoietin. Br. J. Haematol. $141,14-31$.

Arcasoy, M. O. (2010). Non-erythroid effects of erythropoietin. Haematologica 95, 1803-1805.

Arranz-Gibert, P., Guixer, B., Prades, R., Ciudad, S., Giralt, E., and Teixidó, M. (2019). A MALDI-TOFbased Method for Studying the Transport of BBB Shuttles-Enhancing Sensitivity and Versatility of Cell-Based In Vitro Transport Models. Sci. Rep. 9. doi:10.1038/s41598-019-40973-0.

Ashton, R. S., Conway, A., Pangarkar, C., Bergen, J., Lim, K. II, Shah, P., et al. (2012). Astrocytes regulate adult hippocampal neurogenesis through ephrin-B signaling. Nat. Neurosci. 15, 13991406.

Baxter, R. C. (1985). Measurement of growth hormone and prolactin receptor turnover in rat liver. Endocrinology 117, 650-655.

Becker, V., Schilling, M., Bachmann, J., Baumann, U., Raue, A., Maiwald, T., et al. (2010). Covering a broad dynamic range: Information processing at the erythropoietin receptor. Science 328 , 1404-1408. 
Benjamini, Y., and Hochberg, Y. (1995). Controlling the False Discovery Rate - a Practical and Powerful Approach to Multiple Testing. Journal of the Royal Statistical Society Series B-Methodological 1995.pdf. J. R. Stat. Soc. Ser. B 57, 289-300.

Bernaudin, M., Marti, H. H., Roussel, S., Divoux, D., Nouvelot, A., MacKenzie, E. T., et al. (1999). A potential role for erythropoietin in focal permanent cerebral ischemia in mice. J. Cereb. Blood Flow Metab. 19, 643-651.

Bond, W. S., and Rex, T. S. (2014). Evidence that erythropoietin modulates neuroinflammation through differential action on neurons, astrocytes, and microglia. Front. Immunol. 5. doi:10.3389/fimmu.2014.00523.

Bonnas, C. B. (2009). Identification of erythropoietin isoforms and evaluation of their biological importance. Diss. Charité Berlin, 150.

Bonnas, C., Wüstefeld, L., Winkler, D., Kronstein-Wiedemann, R., Dere, E., Specht, K., et al. (2017). $\mathrm{EV}-3$, an endogenous human erythropoietin isoform with distinct functional relevance. Sci. Rep. 7. doi:10.1038/s41598-017-03167-0.

Boulay, J. L., O'Shea, J. J., and Paul, W. E. (2003). Molecular phylogeny within type I cytokines and their cognate receptors. Immunity 19, 159-163.

Bouvier, M., Collins, S., O’Dowd, B. F., Campbell, P. T., De Blasi, A., Kobilka, B. K., et al. (1989). Two distinct pathways for cAMP-mediated down-regulation of the $\beta 2$-adrenergic receptor. Phosphorylation of the receptor and regulation of its mRNA level. J. Biol. Chem. 264, 1678616792.

Brines, M. (2010). The therapeutic potential of erythropoiesis-stimulating agents for tissue protection: A tale of two receptors. Blood Purif. 29, 86-92.

Brines, M., and Cerami, A. (2005). Emerging biological roles for erythropoietin in the nervous system. Nat. Rev. Neurosci. 6, 484. doi: 10.1038/nrn1687. doi:10.1038/nrn1687.

Brines, M., Grasso, G., Fiordaliso, F., Sfacteria, A., Ghezzi, P., Fratelli, M., et al. (2004). Erythropoietin mediates tissue protection through an erythropoietin and common -subunit heteroreceptor. Proc. Natl. Acad. Sci. 101, 14907-14912.

Brines, M., Patel, N. S. A., Villa, P., Brines, C., Mennini, T., De Paola, M., et al. (2008). Nonerythropoietic, tissue-protective peptides derived from the tertiary structure of erythropoietin. Proc. Natl. Acad. Sci. 105, 10925-10930. 
Brown, S. J., Shippy, T. D., Miller, S., Bolognesi, R., Beeman, R. W., Lorenzen, M. D., et al. (2009). The red flour beetle, Tribolium castaneum (Coleoptera): A model for studies of development and pest biology. Cold Spring Harb. Protoc. 4. doi:10.1101/pdb.emo126.

Bucher, G. (2009). The Beetle Book. 34. Available at: http://wwwuser.gwdg.de/ gbucher1/triboliumcastaneum-beetle-book1.pdf.

Bucher, G., Scholten, J., and Klingler, M. (2002). Parental RNAi in tribolium (coleoptera). Curr. Biol. 12, 85-86.

Calvillo, L., Latini, R., Kajstura, J., Leri, A., Anversa, P., Ghezzi, P., et al. (2003). Recombinant human erythropoietin protects the myocardium from ischemia-reperfusion injury and promotes beneficial remodeling. Proc. Natl. Acad. Sci. U. S. A. 100, 4802-4806.

Castillo, C., Fernández-Mendívil, C., Buendia, I., Saavedra, P., Meza, C., Parra, N. C., et al. (2019). Neuroprotective effects of EpoL against oxidative stress induced by soluble oligomers of $A \beta$ peptide. Redox Biol. 24, 101187. doi:10.1016/j.redox.2019.101187.

Cavadas, M. A. S., Cheong, A., and Taylor, C. T. (2017). The regulation of transcriptional repression in hypoxia. Exp. Cell Res. 356, 173-181.

Chamorro, M. E., Wenker, S. D., Vota, D. M., Vittori, D. C., and Nesse, A. B. (2013). Signaling pathways of cell proliferation are involved in the differential effect of erythropoietin and its carbamylated derivative. Biochim. Biophys. Acta - Mol. Cell Res. 1833, 1960-1968.

Chao, S. H., and Nagoshi, R. N. (1999). Induction of apoptosis in the germline and follicle layer of Drosophila egg chambers. Mech. Dev. 88, 159-172.

Chateauvieux, S., Grigorakaki, C., Morceau, F., Dicato, M., and Diederich, M. (2011). Erythropoietin, erythropoiesis and beyond. Biochem. Pharmacol. 82, 1291-1303.

Chattong, S., Tanamai, J., Kiatsomchai, P., Nakatsu, M., Sereemaspun, A., Pimpha, N., et al. (2013). Non-erythropoietic EPO protects kidney in I/R. Br. J. Pharmacol. 168, 189-199.

Chen, G. L., Yang, L., Rowe, T. C., Halligan, B. D., Tewey, K. M., and Liu, L. F. (1984). Nonintercalative antitumor drugs interfere with the breakage-reunion reaction of mammalian DNA topoisomerase II. J. Biol. Chem. 259, 13560-13566.

Chen, Z. Y., Asavaritikrai, P., Prchal, J. T., and Noguchi, C. T. (2007). Endogenous erythropoietin signaling is required for normal neural progenitor cell proliferation. J. Biol. Chem. 282, 2587525883. 
Cirulli, E. T., Lasseigne, B. N., Petrovski, S., Sapp, P. C., Dion, P. A., Leblond, C. S., et al. (2015). Exome sequencing in amyotrophic lateral sclerosis identifies risk genes and pathways. Science 347, $1436-1441$.

Clifford, B., Beljin, M., Stark, G. R., and Taylor, W. R. (2003). G2 arrest in response to topoisomerase II inhibitors: The role of p53. Cancer Res. 63, 4074-4081.

Cokic, V. P., Bhattacharya, B., Beleslin-Cokic, B. B., Noguchi, C. T., Puri, R. K., and Schechter, A. N. (2012). JAK-STAT and AKT pathway-coupled genes in erythroid progenitor cells through ontogeny. J. Transl. Med. 10. doi:10.1186/1479-5876-10-116.

Constantinescu, S. N., Ghaffari, S., and Lodish, H. F. (1999). The erythropoietin receptor: Structure, activation and intracellular signal transduction. Trends Endocrinol. Metab. 10, 18-23.

Cortés, F., Pastor, N., Mateos, S., and Domínguez, I. (2003). Roles of DNA topoisomerases in chromosome segregation and mitosis. Mutat. Res. - Rev. Mutat. Res. 543, 59-66.

Dahlen, D. D., Broudy, V. C., and Drachman, J. G. (2003). Internalization of the thrombopoietin receptor is regulated by 2 cytoplasmic motifs. Blood 102, 102-108.

Dame, C., Juul, S. E., and Christensen, R. D. (2001). The biology of erythropoietin in the central nervous system and its neurotrophic and neuroprotective potential. Biol. Neonate 79, 228-235.

Darzynkiewicz, Z., Juan, G., Li, X., Gorczyca, W., Murakami, T., and Traganos, F. (1997). Cytometry in cell necrobiology: Analysis of apoptosis and accidental cell death (necrosis). Cytometry 27, 1-20. doi:10.1002/(SICI)1097-0320(19970101)27:1<1::AID-CYTO2>3.0.CO;2-L.

De Sousa Abreu, R., Penalva, L. O., Marcotte, E. M., and Vogel, C. (2009). Global signatures of protein and mRNA expression levels. Mol. Biosyst. 5, 1512-1526.

Ding, J., Wang, J., Li, Q. Y., Yu, J. Z., Ma, C. G., Wang, X., et al. (2017). Neuroprotection and CD131/GDNF/AKT Pathway of Carbamylated Erythropoietin in Hypoxic Neurons. Mol. Neurobiol. 54, 5051-5060.

Dmytriyeva, O., Pankratova, S., Korshunova, I., and Walmod, P. S. (2016). Epobis is a Nonerythropoietic and Neuroprotective Agonist of the Erythropoietin Receptor with AntiInflammatory and Memory Enhancing Effects. Mediators Inflamm. 2016. doi:10.1155/2016/1346390.

Donahaye, E. (1990). Laboratory selection of resistance by the red flour beetle, Tribolium castaneum (Herbst), to an atmosphere of low oxygen concentration. Phytoparasitica 18, 189-202. 
Dönitz, J., Schmitt-Engel, C., Grossmann, D., Gerischer, L., Tech, M., Schoppmeier, M., et al. (2015). iBeetle-Base: A database for RNAi phenotypes in the red flour beetle Tribolium castaneum. Nucleic Acids Res. 43, D720-D725.

Eddabra, L., Wenner, T., El Btaouri, H., Baranek, T., Madoulet, C., Cornillet-Lefebvre, P., et al. (2012). Arginine 482 to glycine mutation in ABCG2/BCRP increases etoposide transport and resistance to the drug in HEK-293 cells. Oncol. Rep. 27, 232-237.

Ehrenreich, H., Hasselblatt, M., Dembowski, C., Cepek, L., Lewczuk, P., Stiefel, M., et al. (2002). Erythropoietin Therapy for Acute Stroke Is Both Safe and Beneficial. Mol. Med. 8, 495-505.

Ehrenreich, H., Weissenborn, K., Prange, H., Schneider, D., Weimar, C., Wartenberg, K., et al. (2009). Recombinant human erythropoietin in the treatment of acute ischemic stroke. Stroke 40. doi:10.1161/STROKEAHA.109.564872.

Elliott, S., Lorenzini, T., Chang, D., Barzilay, J., and Delorme, E. (1997). Mapping of the active site of recombinant human erythropoietin. Blood 89, 493-502.

Encinas, M., Iglesias, M., Liu, Y., Wang, H., Muhaisen, A., Cena, V., et al. (2000). Sequential Treatment of SH-SY5Y Cells with Retinoic Acid and Brain-Derived Neurotrophic Factor Gives Rise to Fully Differentiated, Neurotrophic Factor-Dependent, Human Neuron-Like Cells. J. Neurochem. 75, 991-1003.

Eng, W. K., Faucette, L., Johnson, R. K., and Sternglanz, R. (1988). Evidence that DNA topoisomerase I is necessary for the cytotoxic effects of camptothecin. Mol. Pharmacol. 34, 755-760.

Erbayraktar, S., Grasso, G., Sfacteria, A., Xie, Q. Q. -w., Coleman, T., Kreilgaard, M., et al. (2003). Asialoerythropoietin is a nonerythropoietic cytokine with broad neuroprotective activity in vivo. Proc. Natl. Acad. Sci. 100, 6741-6746.

Erbayraktar, Z., Erbayraktar, S., Yilmaz, O., Cerami, A., Coleman, T., and Brines, M. (2009). Nonerythropoietic tissue protective compounds are highly effective facilitators of wound healing. Mol. Med. 15, 235-241.

Erslev, A. (1953). Humoral Regulation of Red Cell Production. Blood 8, 349-357.

Esquerré, T., Laguerre, S., Turlan, C., Carpousis, A. J., Girbal, L., and Cocaign-Bousquet, M. (2014). Dual role of transcription and transcript stability in the regulation of gene expression in Escherichia coli cells cultured on glucose at different growth rates. Nucleic Acids Res. 42, 24602472. 
Fernando, G., Yamila, R., Cesar, G. J., and Ramón, R. (2018). Neuroprotective effects of neuroEPO using an in vitro model of stroke. Behav. Sci. (Basel). 8. doi:10.3390/bs8020026.

Fu, M., Wang, C., Li, Z., Sakamaki, T., and Pestell, R. G. (2004). Minireview: Cyclin D1: Normal and abnormal functions. Endocrinology 145, 5439-5447.

Genc, S., Koroglu, T. F., and Genc, K. (2004). Erythropoietin and the nervous system. Brain Res. 1000, $19-31$.

Genty, N., Paly, J., Edery, M., Kelly, P. A., Djiane, J., and Salesse, R. (1994). Endocytosis and degradation of prolactin and its receptor in Chinese hamster ovary cells stably transfected with prolactin receptor cDNA. Mol. Cell. Endocrinol. 99, 221-228.

Ghezzi, P., and Brines, M. (2004). Erythropoietin as an antiapoptotic, tissue-protective cytokine. Cell Death Differ. 11, 37-44.

Gocht, D., Wagner, S., and Heinrich, R. (2009). Recognition, Presence, and Survival of Locust Central Nervous Glia In Situ and In Vitro. Microsc. Res. Tech. 72, 385-397.

Gorin, E., and Goodman, H. M. (1985). Turnover of growth hormone receptors in rat adipocytes. Endocrinology 116, 1796-1805.

Götz, S., García-Gómez, J. M., Terol, J., Williams, T. D., Nagaraj, S. H., Nueda, M. J., et al. (2008). Highthroughput functional annotation and data mining with the Blast2GO suite. Nucleic Acids Res. 36, 3420-3435.

Greenberg, S., and Ar, A. (1996). Effects of chronic hypoxia, normoxia and hyperoxia on larval development in the beetle Tenebrio molitor. J. Insect Physiol. 42, 991-996.

Guarino, A. M., Mauro, G. Di, Ruggiero, G., Geyer, N., Delicato, A., Foulkes, N. S., et al. (2019). YB-1 recruitment to stress granules in zebrafish cells reveals a differential adaptive response to stress. Sci. Rep. 9. doi:10.1038/s41598-019-45468-6.

Gygi, S. P., Rochon, Y., Franza, B. R., and Aebersold, R. (1999). Correlation between Protein and mRNA Abundance in Yeast. Mol. Cell. Biol. 19, 1720-1730.

Hacke, W., Donnan, G., Fieschi, C., Kaste, M., von Kummer, R., Broderick, J., et al. (2004). Association of outcome with early stroke treatment: Pooled analysis of ATLANTIS, ECASS, and NINDS rt-PA stroke trials.

Hahm, S. H., Chung, J. H., Agustina, L., Han, S. H., Yoon, I. S., Park, J. H., et al. (2012). Human MutY 
homolog induces apoptosis in etoposide-treated HEK293 cells. Oncol. Lett. 4, 1203-1208.

Hahn, N., Büschgens, L., Schwedhelm-domeyer, N., Bank, S., Geurten, B. R. H., Neugebauer, P., et al. (2019). The Orphan Cytokine Receptor CRLF3 Emerged With the Origin of the Nervous System and Is a Neuroprotective Erythropoietin Receptor in Locusts. Front. Mol. Neurosci. 12. doi:10.3389/fnmol.2019.00251.

Hahn, N., Knorr, D., Liebig, J., Wüstefeld, L., Peters, K., Büscher, M., et al. (2017). The human orphan cytokine receptor CRLF3 is a neuroprotective erythropoietin receptor in insects. Front. Mol. Neurosci. 10. doi:10.3389/fnmol.2017.00223.

Han, C., Jan, L. Y., and Jan, Y. N. (2011). Enhancer-driven membrane markers for analysis of nonautonomous mechanisms reveal neuron-glia interactions in Drosophila. Proc. Natl. Acad. Sci. U. S. A. 108, 9673-9678.

Hartenstein, V. (2011). Morphological diversity and development of glia in Drosophila. Glia 59, 12371252.

Hashimoto, Y., Muramatsu, K., Kunii, M., Yoshimura, S. I., Yamada, M., Sato, T., et al. (2012). Uncovering genes required for neuronal morphology by morphology-based gene trap screening with a revertible retrovirus vector. FASEB J. 26, 4662-4674.

He, B., Buescher, M., Farnworth, M. S., Strobl, F., Stelzer, E., Dieter, N., et al. (2019). An ancestral apical brain region contributes to the central complex under the control of foxQ2 in the beetle Tribolium. Elife 8. doi:10.7554/eLife.49065.

Heinrich, R., Günther, V., and Miljus, N. (2017). Erythropoietin-Mediated Neuroprotection in Insects Suggests a Prevertebrate Evolution of Erythropoietin-Like Signaling. Vitam. Horm. 105, 181196.

Heir, P., Srikumar, T., Bikopoulos, G., Bunda, S., Poon, B. P., Lee, J. E., et al. (2016). Oxygen-dependent regulation of erythropoietin receptor turnover and signaling. J. Biol. Chem. 291, 7357-7372.

Heiss, W. -D, and Rosner, G. (1983). Functional recovery of cortical neurons as related to degree and duration of ischemia. Ann. Neurol. 14, 294-301.

Henderson, J. T., Georgiou, J., Jia, Z., Robertson, J., Elowe, S., Roder, J. C., et al. (2001). The receptor tyrosine kinase EphB2 regulates NMDA-dependent synaptic function. Neuron 32, 1041-1056.

Hendig, D., Langmann, T., Kocken, S., Zarbock, R., Szliska, C., Schmitz, G., et al. (2008). Gene expression profiling of $A B C$ transporters in dermal fibroblasts of pseudoxanthoma elasticum 
patients identifies new candidates involved in PXE pathogenesis. Lab. Investig. 88, 1303-1315.

Hothorn, T., Hornik, K., Wiel, M. A. van de, and Zeileis, A. (2006). A Lego System for Conditional Inference. The American Statistician. Am. Stat. 60, 257-263.

Hothorn, T., Hornik, K., Wiel, M. A. van de, and Zeileis, A. (2008). Implementing a Class of Permutation Tests: The coin Package. J. Stat. Softw. 28. doi:10.18637/jss.v028.i08.

Hunnekuhl, V. S., Siemanowski, J., M.S., F., He, B., and Bucher, G. (2020). "Immunohistochemistry and Fluorescent Whole Mount RNA In Situ Hybridization in Larval and Adult Brains of Tribolium," in Brain Development, ed. S. Sprecher (New York, NY: Humana).

Iwaki, T., Figuera, M., Ploplis, V. A., and Castellino, F. J. (2003). Rapid selection of Drosophila S2 cells with the puromycin resistance gene. Biotechniques 35, 482-486.

Jayasooriya, R. G. P. T., Dilshara, M. G., Molagoda, I. M. N., Park, C., Park, S. R., Lee, S., et al. (2018). Camptothecin induces $\mathrm{G} 2 / \mathrm{M}$ phase arrest through the ATM-Chk2- Cdc25C axis as a result of autophagy-induced cytoprotection: Implications of reactive oxygen species. Oncotarget 9, 21744-21757.

Jelkmann, W. (1992). Erythropoietin: structure, control of production, and function. Physiol. Rev. 72, 449-489.

Kadota, T., Shingo, T., Yasuhara, T., Tajiri, N., Kondo, A., Morimoto, T., et al. (2009). Continuous intraventricular infusion of erythropoietin exerts neuroprotective/rescue effects upon Parkinson's disease model of rats with enhanced neurogenesis. Brain Res. 1254, 120-127.

Kaiser, A., Hartzendorf, S., Wobschall, A., and Hetz, S. K. (2010). Modulation of cyclic CO2 release in response to endogenous changes of metabolism during pupal development of Zophobas rugipes (Coleoptera: Tenebrionidae). J. Insect Physiol. 56, 502-512.

Kanehisa, M., and Goto, S. (2000). KEGG: Kyoto encyclopedia of genes and genomes. Nucleic Acids Res. 28, 27-30.

Kathrein, K. L., Lorenz, R., Innes, A. M., Griffiths, E., and Winandy, S. (2005). Ikaros Induces Quiescence and T-Cell Differentiation in a Leukemia Cell Line. Mol. Cell. Biol. 25, 1645-1654.

Kawakami, M., Sekiguchi, M., Sato, K., Kozaki, S., and Takahashi, M. (2001). Erythropoietin Receptormediated Inhibition of Exocytotic Glutamate Release Confers Neuroprotection during Chemical Ischemia. J. Biol. Chem. 276, 39469-39475. 
Kharel, K., Mason, L. J., Murdock, L. L., and Baributsa, D. (2019). Efficacy of Hypoxia Against Tribolium castaneum (Coleoptera: Tenebrionidae) Throughout Ontogeny. J. Econ. Entomol. 112, 14631468.

Kittur, F. S., Lin, Y., Arthur, E., Hung, C. Y., Li, P. A., Sane, D. C., et al. (2019). Recombinant asialoerythropoetin protects HL-1 cardiomyocytes from injury via suppression of Mst1 activation. Biochem. Biophys. Reports 17, 157-168.

Klingmüller, U., Bergelson, S., Hsiao, J. G., and Lodish, H. F. (1996). Multiple tyrosine residues in the cytosolic domain of the erythropoietin receptor promote activation of STAT5. Proc. Natl. Acad. Sci. U. S. A. 93, 8324-8328.

Koerte, S., Keesey, I. W., Khallaf, M. A., Cortés Llorca, L., Grosse-Wilde, E., Hansson, B. S., et al. (2018). Evaluation of the DREAM Technique for a High-Throughput Deorphanization of Chemosensory Receptors in Drosophila. Front. Mol. Neurosci. 11. doi:10.3389/fnmol.2018.00366.

Koh, C. M., Gurel, B., Sutcliffe, S., Aryee, M. J., Schultz, D., Iwata, T., et al. (2011). Alterations in nucleolar structure and gene expression programs in prostatic neoplasia are driven by the MYC oncogene. Am. J. Pathol. 178, 1824-1834.

Koniszewski, N. D. B., Kollmann, M., Bigham, M., Farnworth, M., He, B., Büscher, M., et al. (2016). The insect central complex as model for heterochronic brain development-background, concepts, and tools. Dev. Genes Evol. 226, 209-219.

Korecka, J. A., van Kesteren, R. E., Blaas, E., Spitzer, S. O., Kamstra, J. H., Smit, A. B., et al. (2013). Phenotypic Characterization of Retinoic Acid Differentiated SH-SY5Y Cells by Transcriptional Profiling. PLoS One 8, e63862. doi:10.1371/journal.pone.0063862.

Kramer, N. J., Haney, M. S., Morgens, D. W., Jovičić, A., Couthouis, J., Li, A., et al. (2018). CRISPR-Cas9 screens in human cells and primary neurons identify modifiers of C9ORF72 dipeptide-repeatprotein toxicity. Nat. Genet. 50, 603-612.

Kranenburg, O., van der Eb, A. J., and Zantema, A. (1996). Cyclin D1 is an essential mediator of apoptotic neuronal cell death. EMBO J. 15, 46-54.

Kretz, A., Happold, C. J., Marticke, J. K., and Isenmann, S. (2005). Erythropoietin promotes regeneration of adult CNS neurons via Jak2/Stat3 and PI3K/AKT pathway activation. Mol. Cell. Neurosci. 29, 569-579. 
Lackner, D. H., Schmidt, M. W., Wu, S., Wolf, D. A., and Bähler, J. (2012). Regulation of transcriptome, translation, and proteome in response to environmental stress in fission yeast. Genome Biol. 13, R25. doi:10.1186/gb-2012-13-4-r25.

Lagarto, A., Bueno, V., Guerra, I., Valdés, O., Couret, M., López, R., et al. (2011). Absence of hematological side effects in acute and subacute nasal dosing of erythropoietin with a low content of sialic acid. Exp. Toxicol. Pathol. 63, 563-567.

Lang, K. J. D., Kappel, A., and Goodall, G. J. (2002). Hypoxia-inducible Factor-1alpha mRNA Contains an Internal Ribosome Entry Site That Allows Efficient Translation during Normoxia and Hypoxia. Mol. Biol. Cell 13, 1792-1801.

Langenheim, J. F., and Chen, W. Y. (2009). Development of a novel ligand that activates JAK2/STAT5 signaling through a heterodimer of prolactin receptor and growth hormone receptor. J. Recept. Signal Transduct. 29, 107-112.

Langmead, B., and Salzberg, S. L. (2012). Fast gapped-read alignment with Bowtie 2. Nat. Methods 9, 357-359.

Lee, M. V., Topper, S. E., Hubler, S. L., Hose, J., Wenger, C. D., Coon, J. J., et al. (2011). A dynamic model of proteome changes reveals new roles for transcript alteration in yeast. Mol. Syst. Biol. 7. doi:10.1038/msb.2011.48.

Lee, S. T., Chu, K., Park, J. E., Jung, K. H., Jeon, D., Lim, J. Y., et al. (2012). Erythropoietin improves memory function with reducing endothelial dysfunction and amyloid-beta burden in Alzheimer's disease models. J. Neurochem. 120, 115-124.

Lee, T., and Luo, L. (1999). Mosaic Analysis with a Repressible Neurotechnique Cell Marker for Studies of Gene Function in Neuronal Morphogenesis. Neuron 22, 451-461.

Lee, T. S., Lu, K. Y., Yu, Y. Bin, Lee, H. Te, and Tsai, F. C. (2015). $\beta$ Common Receptor Mediates Erythropoietin-Conferred Protection on OxLDL-Induced Lipid Accumulation and Inflammation in Macrophages. Mediators Inflamm. 439759. doi:10.1155/2015/439759.

Leist, M., Ghezzi, P., Grasso, G., Bianchi, R., Villa, P., Fratelli, M., et al. (2004). Derivatives of Erythropoietin That Are Tissue Protective But Not Erythropoietic. Science 305, 239-243.

Leung, K. C., Waters, M. J., Markus, I., Baumbach, W. R., and Ho, K. K. Y. (1997). Insulin and insulinlike growth factor-I acutely inhibit surface translocation of growth hormone receptors in osteoblasts: A novel mechanism of growth hormone receptor regulation. Proc. Natl. Acad. Sci. 


\section{U. S. A. $94,11381-11386$.}

Liongue, C., Taznin, T., and Ward, A. C. (2016). Signaling via the CytoR/JAK/STAT/SOCS pathway: Emergence during evolution. Mol. Immunol. 71, 166-175.

Liongue, C., and Ward, A. C. (2007). Evolution of Class I cytokine receptors. BMC Evol. Biol. 7. doi:10.1186/1471-2148-7-120.

Liu, L., and Simon, M. C. (2004). Regulation of transcription and translation by hypoxia. Cancer Biol. Ther. 3, 492-497.

Liu, T., Zeng, X., Sun, F., Hou, H., Guan, Y., Guo, D., et al. (2017). EphB4 Regulates Self-Renewal, Proliferation and Neuronal Differentiation of Human Embryonic Neural Stem Cells in Vitro. Cell. Physiol. Biochem. 41, 819-834.

Liu, Y., Beyer, A., and Aebersold, R. (2016). On the Dependency of Cellular Protein Levels on mRNA Abundance. Cell 165, 535-550.

Liu, Y., Perdreau, S. A., Chatterjee, P., Wang, L., Kuan, S.-F., and Duensing, A. (2008). Imatinib Mesylate Induces Quiescence in Gastrointestinal Stromal Tumor Cells through the CDH1-SKP2p27Kip1 Signaling Axis. Cancer Res. 68, 9015-9023.

Livak, K. J., and Schmittgen, T. D. (2001). Analysis of relative gene expression data using real-time quantitative PCR and the 2- $\Delta \Delta C T$ method. Methods 25, 402-408.

Livnah, O., Stura, E. A., Johnson, D. L., Middleton, S. A., Mulcahy, L. S., Wrighton, N. C., et al. (1996). Functional mimicry of a protein hormone by a peptide agonist: The EPO receptor complex at 2.8 Å. Science $273,464-471$.

Lopes, F. M., Schröder, R., Júnior, M. L. C. da F., Zanotto-Filho, A., Müller, C. B., Pires, A. S., et al. (2010). Comparison between proliferative and neuron-like SH-SY5Y cells as an in vitro model for Parkinson disease studies. Brain Res. 1337, 85-94.

Loudon, C. (1988). Development of Tenebrio molitor in low oxygen levels. J. Insect Physiol. 34, 97103.

Love, M. I., Huber, W., and Anders, S. (2014). Moderated estimation of fold change and dispersion for RNA-seq data with DESeq2. Genome Biol. 15. doi:10.1186/s13059-014-0550-8.

Maden, M. (2007). Retinoic acid in the development, regeneration and maintenance of the nervous system. Nat. Rev. Neurosci. 8, 755-765. 
Mangiafico, S. (2019). rcompanion: Functions to Support Extension Education Program Evaluation. Available at: https://cran.r-project.org/package=rcompanion\%0A\%0A.

Marguerat, S., Schmidt, A., Codlin, S., Chen, W., Aebersold, R., and Bähler, J. (2012). Quantitative analysis of fission yeast transcriptomes and proteomes in proliferating and quiescent cells. Cell $151,671-683$.

Marler, J. R., Tilley, B. C., Lu, M., Brott, T. G., Lyden, P. C., Grotta, J. C., et al. (2000). Early stroke treatment associated with better outcome: The NINDS rt-PA Stroke Study. Neurology 55, 16491655.

Matthews, D. J., Topping, R. S., Cass, R. T., and Giebel, L. B. (1996). A sequential dimerization mechanism for erythropoietin receptor activation. Proc. Natl. Acad. Sci. U. S. A. 93, 9471-6.

McCarthy, D. J., Chen, Y., and Smyth, G. K. (2012). Differential expression analysis of multifactor RNASeq experiments with respect to biological variation. Nucleic Acids Res. 40, 4288-4297.

Mennini, T., De Paola, M., Bigini, P., Mastrotto, C., Fumagalli, E., Barbera, S., et al. (2006). Nonhematopoietic erythropoietin derivatives prevent motoneuron degeneration in vitro and in vivo. Mol. Med. 12, 153-160. doi:10.2119/2006-00045.Mennini.

Meyer, S., Temme, C., and Wahle, E. (2004). Messenger RNA turnover in eukaryotes: Pathways and enzymes. Crit. Rev. Biochem. Mol. Biol. 39, 197-216.

Miljus, N., Heibeck, S., Jarrar, M., Micke, M., Ostrowski, D., Ehrenreich, H., et al. (2014). Erythropoietin-mediated protection of insect brain neurons involves JAK and STAT but not PI3K transduction pathways. Neuroscience 258, 218-227.

Miljus, N., Massih, B., Weis, M. A., Rison, J. V., Bonnas, C. B., Sillaber, I., et al. (2017). Neuroprotection and endocytosis: erythropoietin receptors in insect nervous systems. J. Neurochem. 141, 63-74.

Miller, J. L., Church, T. J., Leonoudakis, D., Lariosa-Willingham, K., Frigon, N. L., Tettenborn, C. S., et al. (2015). Discovery and Characterization of Nonpeptidyl Agonists of the Tissue-Protective Erythropoietin Receptor. Mol. Pharmacol. 88, 357-367.

Mitkovski, M., Dahm, L., Heinrich, R., Monnheimer, M., Gerhart, S., Stegmüller, J., et al. (2015). Erythropoietin dampens injury-induced microglial motility. J. Cereb. Blood Flow Metab. 35, $1233-1236$.

Moffat, J., Grueneberg, D. A., Yang, X., Kim, S. Y., Kloepfer, A. M., Hinkle, G., et al. (2006). A Lentiviral RNAi Library for Human and Mouse Genes Applied to an Arrayed Viral High-Content Screen. Cell 
$124,1283-1298$.

Moon, C., Krawczyk, M., Paik, D., Coleman, T., Brines, M., Juhaszova, M., et al. (2006). Erythropoietin, modified to not stimulate red blood cell production, retains its cardioprotective properties. $J$. Pharmacol. Exp. Ther. 316, 999-1005.

Moore, E., and Bellomo, R. (2011). Erythropoietin ( EPO ) in acute kidney injury. Ann. Intensive Care 1. doi:10.1186/2110-5820-1-3.

Morishita, E., Masuda, S., Nagao, M., Yasuda, Y., and Sasaki, R. (1996). Erythropoetin receptor is expressed in rat hippocampal and cerebral cortical neurons, and erythropoietin prevents in vitro glutamate-induced neuronal death. Neuroscience 76, 105-116.

Murray, C. J. L., and Lopez, A. D. (1997). Mortality by cause for eight regions of the world: Global Burden of Disease Study. Lancet 349, 1269-1276.

Muslimovic, A., Nyström, S., Gao, Y., and Hammarsten, O. (2009). A next-generation approach to the characterization of a non-model plant transcriptome. PLoS One 4, e5859. doi:10.1371/ journal.pone.0005859.

Nadam, J., Navarro, F., Sanchez, P., Moulin, C., Georges, B., Laglaine, A., et al. (2007). Neuroprotective effects of erythropoietin in the rat hippocampus after pilocarpine-induced status epilepticus. Neurobiol. Dis. 25, 412-426.

Navarro, S., and Donahaye, E. (1990). Generation and application of modified atmospheres and fumigants for the control of storage insects. Fumigation Control. Atmos. Storage Grain Proc. an Int. Conf. Proceeding, 152-163.

Neumann, D., Wikstrom, L., Watowich, S. S., and Lodish, H. F. (1993). Intermediates in degradation of the erythropoietin receptor accumulate and are degraded in lysosomes. J. Biol. Chem. 268, 13639-13649.

Noguchi, C. T., Asavaritikrai, P., Teng, R., and Jia, Y. (2007). Role of erythropoietin in the brain. Crit. Rev. Oncol. Hematol. 64, 159-171.

Odell, J. P. (1998). Energetics of metamorphosis in two holometabolous insect species: Manduca sexta (Lepidoptera: Sphingidae) and Tenebrio molitor (Coleoptera: Tenebrionidae). J. Exp. Zool. 280, 344-353.

Ogunshola, O. O., and Bogdanova, A. Y. (2013). Tissue-Protective Cytokines. Methods Mol. Biol. 982, $13-41$. 
Ostrowski, D., Ehrenreich, H., and Heinrich, R. (2011). Erythropoietin promotes survival and regeneration of insect neurons in vivo and in vitro. Neuroscience 188, 95-108.

Ostrowski, D., and Heinrich, R. (2018). Alternative Erythropoietin Receptors in the Nervous System. J. Clin. Med. 7. doi:10.3390/jcm7020024.

Ott, C., Martens, H., Hassouna, I., Oliveira, B., Erck, C., Zafeiriou, M. P., et al. (2015). Widespread expression of erythropoietin receptor in brain and its induction by injury. Mol. Med. 21, 803815.

Ozkurt, M., Uzuner, K., Erkasap, N., Kus, G., Ozyurt, R., Uysal, O., et al. (2018). Erythropoietin Protects the Kidney by Regulating the Effect of TNF- $\alpha$ in L-NAME-Induced Hypertensive Rats. Kidney Blood Press. Res. 43, 807-819.

Pankratova, S., Gu, B., Kiryushko, D., Korshunova, I., Køhler, L. B., Rathje, M., et al. (2012). A new agonist of the erythropoietin receptor, Epobis, induces neurite outgrowth and promotes neuronal survival. J. Neurochem. 121, 915-923.

Pankratova, S., Kiryushko, D., Sonn, K., Soroka, V., Køhler, L. B., Rathje, M., et al. (2010). Neuroprotective properties of a novel, non-haematopoietic agonist of the erythropoietin receptor. Brain 133, 2281-2294.

Park, H. D., and Buck, J. (1960). The relation of oxygen consumption to ambient oxygen concentration during metamorphosis of the blowfly, Phormia regina. J. Insect Physiol. 4, 220-228.

Pecquet, C., Diaconu, C. C., Staerk, J., Girardot, M., Marty, C., Royer, Y., et al. (2012). Thrombopoietin receptor down-modulation by JAK2 V617F: Restoration of receptor levels by inhibitors of pathologic JAK2 signaling and of proteasomes. Blood 119, 4625-4635.

Pedroso, I., Garcia, M., Casabona, E., Morales, L., Bringas, M. L., Pérez, L., et al. (2018). Protective activity of erythropoyetine in the cognition of patients with Parkinson's disease. Behav. Sci. (Basel). 8. doi:10.3390/bs8050051.

Perkin, L. C., and Oppert, B. (2019). Gene expression in Tribolium castaneum life stages: Identifying a species-specific target for pest control applications. PeerJ 7, e6946. doi:10.7717/peerj.6946.

Piiroinen, S., Lindström, L., and Lyytinen, A. (2010). Resting metabolic rate can vary with age independently from body mass changes in the Colorado potato beetle, Leptinotarsa decemlineata. J. Insect Physiol. 56, 277-282.

Posnien, N., Koniszewski, N. D. B., Hein, H. J., and Bucher, G. (2011). Candidate gene screen in the red 
flour beetle tribolium reveals six3 as ancient regulator of anterior median head and central complex development. PLoS Genet. 7. doi:10.1371/journal.pgen.1002416.

Powers, W. J., Rabinstein, A. A., Ackerson, T., Adeoye, O. M., Bambakidis, N. C., Becker, K., et al. (2018). 2018 Guidelines for the Early Management of Patients With Acute Ischemic Stroke. doi:10.1161/STR.0000000000000158.

Pradeep, S., Huang, J., Mora, E. M., Nick, A. M., Cho, M. S., Wu, S. Y., et al. (2015). Erythropoietin Stimulates Tumor Growth via EphB4. Cancer Cell 28, 610-622.

Pype, J. L., Mak, J. C. W., Dupont, L. J., Verleden, G. M., and Barnes, P. J. (1998). Desensitization of the histamine H1-receptor and transcriptional down-regulation of histamine H1-receptor gene expression in bovine tracheal smooth muscle. Br. J. Pharmacol. 125, 1477-1484.

R_Core_Team (2019). R: A Language and Environment for Statistical Computing. in (R Foundation for Statistical Computing, Vienna, Austria, https://www.r-project.org/). Available at: https://www.r-project.org/.

Raser, J. M., and O'Shea, E. (2005). Noise in Gene Expression: Origins, Consequences, and Control. Science 309, 2010-2014.

Richards, S., Gibbs, R. A., Weinstock, G. M., Brown, S. J., Denell, R., Beeman, R. W., et al. (2008). The genome of the model beetle and pest Tribolium castaneum. Nature 452, 949-955.

Robinson, M. D., McCarthy, D. J., and Smyth, G. K. (2009). edgeR: A Bioconductor package for differential expression analysis of digital gene expression data. Bioinformatics 26, 139-140.

Rodríguez Cruz, Y., Mengana Támos, Y., Muñoz Cernuda, A., Subirós Martines, N., González-Quevedo, A., Sosa Testé, I., et al. (2010). Treatment with nasal neuro-EPO improves the neurological, cognitive, and histological state in a gerbil model of focal ischemia. ScientificWorldJournal. 10, $2288-2300$.

Roué, G., Pichereau, V., Lincet, H., Colomer, D., and Sola, B. (2008). Cyclin D1 mediates resistance to apoptosis through upregulation of molecular chaperones and consequent redistribution of cell death regulators. Oncogene $27,4909-4920$.

Rousell, J., Haddad, E.-B., Mak, J. C. W., and Barnes, P. J. (1995). Transcriptional down-regulation of m2 muscarinic receptor gene expression in human embryonic lung (HEL 299) cells by protein kinase C*. J. Biol. Chem. 270, 7213-7218.

Royer, Y., Staerk, J., Costuleanu, M., Courtoy, P. J., and Constantinescu, S. N. (2005). Janus kinases 
affect thrombopoietin receptor cell surface localization and stability. J. Biol. Chem. 280, 2725127261.

RStudio_Team (2018). RStudio: Integrated Development for R. in (RStudio, Inc., Boston, MA). Available at: http://www.rstudio.com/.

Sanchez, P. E., Fares, R. P., Risso, J.-J., Bonnet, C., Bouvard, S., Le-Cavorsin, M., et al. (2009). Optimal neuroprotection by erythropoietin requires elevated expression of its receptor in neurons. Proc. Natl. Acad. Sci. 106, 9848-9853.

Sankaran, V. G., Ludwig, L. S., Sicinska, E., Xu, J., Bauer, D. E., Eng, J. C., et al. (2012). Cyclin D3 coordinates the cell cycle during differentiation to regulate erythrocyte size and number. Genes Dev. 26, 2075-2087.

Saucier, C., and Albert, P. R. (1997). Identification of an Endogenous 5-Hydroxytryptamine2A Receptor in NIH-3T3 Cells: Agonist-Induced Down-Regulation Involves Decreases in Receptor RNA and Number. J. Neurochem. 68, 1998-2011.

Saur, S. J., Sangkhae, V., Geddis, A. E., Kaushansky, K., and Hitchcock, I. S. (2010). Ubiquitination and degradation of the thrombopoietin receptor c-Mpl. Blood 115, 1254-1263.

Scacheri, P. C., Rozenblatt-Rosen, O., Caplen, N. J., Wolfsberg, T. G., Umayam, L., Lee, J. C., et al. (2004). Short interfering RNAs can induce unexpected and divergent changes in the levels of untargeted proteins in mammalian cells. Proc. Natl. Acad. Sci. U. S. A. 101, 1892-1897.

Schindelin, J., Arganda-Carreras, I., Frise, E., Kaynig, V., Longair, M., Pietzsch, T., et al. (2012). Fiji: An open-source platform for biological-image analysis. Nat. Methods 9, 676-682.

Schinko, J., Posnien, N., Kittelmann, S., Koniszewski, N., and Bucher, G. (2009). Single and double whole-mount in situ hybridization in red flour beetle (Tribolium) embryos. Cold Spring Harb. Protoc. 4. doi:10.1101/pdb.prot5258.

Schmidt, E. K., Fichelson, S., and Feller, S. M. (2004). PI3 kinase is important for Ras, MEK and Erk activation of Epo-stimulated human erythroid progenitors. BMC Biol. 2. doi:10.1186/1741-70072-7.

Schwanhäusser, B., Busse, D., Li, N., Dittmar, G., Schuchhardt, J., Wolf, J., et al. (2011). Global quantification of mammalian gene expression control. Nature 473, 337-342.

Seibel, N. M., Eljouni, J., Nalaskowski, M. M., and Hampe, W. (2007). Nuclear localization of enhanced green fluorescent protein homomultimers. Anal. Biochem. 368, 95-99. 
Selvarajah, J., Elia, A., Carroll, V. A., and Moumen, A. (2015). DNA damage-induced S and G2/M cell cycle arrest requires mTORC2-dependent regulation of Chk1. Oncotarget 6, 427-440.

Shippy, T. D., Coleman, C. M., Tomoyasu, Y., and Brown, S. J. (2009). Concurrent In situ hybridization and antibody staining in red flour beetle (Tribolium) embryos. Cold Spring Harb. Protoc. 4. doi:10.1101/pdb.prot5257.

Shyu, A. B., Greenberg, M. E., and Belasco, J. G. (1989). The c-fos transcript is targeted for rapid decay by two distinct mRNA degradation pathways. Genes Dev. 3, 60-72.

Singh, A. (2011). Negative feedback through mRNA provides the best control of gene-expression noise. IEEE Trans. Nanobioscience 10, 194-200.

Sirén, A. L., Fratelli, M., Brines, M., Goemans, C., Casagrande, S., Lewczuk, P., et al. (2001). Erythropoietin prevents neuronal apoptosis after cerebral ischemia and metabolic stress. Proc. Natl. Acad. Sci. U. S. A. 98, 4044-4049.

Sooklert, K., Chattong, S., Manotham, K., Boonwong, C., Klaharn, I. Y., Jindatip, D., et al. (2016). Cytoprotective effect of glutaraldehyde erythropoietin on HEK293 kidney cells after silver nanoparticle exposure. Int. J. Nanomedicine 11, 597-605.

Souvenir, R., Doycheva, D., Zhang, J., and Tang, J. (2015). Erythropoietin in Stroke Therapy: Friend or Foe. Curr. Med. Chem. 22, 1205-1213.

Steff, A.-M., Fortin, M., Arguin, C., and Hugo, P. (2001). Detection of a Decrease in Green Fluorescent Protein Fluorescence for the Monitoring of Cell Death: An Assay Amenable to High-Throughput Screening Technologies. Cytometry 45, 237-243.

Syed, R. S., Reid, S. W., Li, C., Cheetham, J. C., Aoki, K. H., Liu, B., et al. (1998). Efficiency of signalling through cytokine receptors depends critically on receptor orientation. Nature 395, 511-516.

Szenajch, J., Wcislo, G., Jeong, J. Y., Szczylik, C., and Feldman, L. (2010). The role of erythropoietin and its receptor in growth, survival and therapeutic response of human tumor cells. From clinic to bench - a critical review. Biochim. Biophys. Acta - Rev. Cancer 1806, 82-95.

Teuscher, M., Ströhlein, N., Birkenbach, M., Schultheis, D., and Schoppmeier, M. (2017). TC003132 is essential for the follicle stem cell lineage in telotrophic Tribolium oogenesis. Front. Zool. 14. doi:10.1186/s12983-017-0212-2.

Thomas, M. G., Loschi, M., Desbats, M. A., and Boccaccio, G. L. (2011). RNA granules: the good, the bad and the ugly. Cell. Signal. 23, 324-334. 
Tögel, F. E., Ahlstrom, J. D., Yang, Y., Hu, Z., Zhang, P., and Westenfelder, C. (2016). Carbamylated erythropoietin outperforms erythropoietin in the treatment of AKI-on-CKD and Other AKI models. J. Am. Soc. Nephrol. 27, 3394-3404.

Torres-Ruiz, R., Benítez-Burraco, A., Martínez-Lage, M., Rodríguez-Perales, S., and García-Bellido, P. (2016). Functional genetic characterization by CRISPR-Cas9 of two enhancers of FOXP2 in a child with speech and language impairment. bioRxiv 15 July. doi:10.1101/064196.

Trauner, J., Schinko, J., Lorenzen, M. D., Shippy, T. D., Wimmer, E. A., Beeman, R. W., et al. (2009). Large-scale insertional mutagenesis of a coleopteran stored grain pest, the red flour beetle Tribolium castaneum, identifies embryonic lethal mutations and enhancer traps. BMC Biol. 7. doi:10.1186/1741-7007-7-73.

Tunç, I., and Navarro, S. (1983). Sensitivity of Tribolium castaneum eggs to modified atmosphere. Entomol. Exp. Appl. 34, 221-226.

Um, M., Gross, A. W., and Lodish, H. F. (2007). A "classical" homodimeric erythropoietin receptor is essential for the antiapoptotic effects of erythropoietin on differentiated neuroblastoma $\mathrm{SH}$ SY5Y and pheochromocytoma PC-12 cells. Cell. Signal. 19, 634-645.

Van Kerkhof, P., Govers, R., Alves Dos Santos, C. M., and Strous, G. J. (2000). Endocytosis and degradation of the growth hormone receptor are proteasome-dependent. J. Biol. Chem. 275, 1575-1580.

Wandzioch, E., Edling, C. E., Palmer, R. H., Carlsson, L., and Hallberg, B. (2004). Activation of the MAP kinase pathway by c-Kit is $\mathrm{PI}-3$ kinase dependent in hematopoietic progenitor/stem cell lines. Blood 104, 51-57.

Wang, L., Cui, S., Liu, Z., Ping, Y., Qiu, J., and Geng, X. (2018). Inhibition of mitochondrial respiration under hypoxia and increased antioxidant activity after reoxygenation of Tribolium castaneum. PLoS One 13, e0199056. doi:10.1371/journal.pone.0199056.

Wang, X., Zhu, C., Wang, X., Gerwien, J. G., Schrattenholz, A., Sandberg, M., et al. (2004). The nonerythropoietic asialoerythropoietin protects against neonatal hypoxia-ischemia as potently as erythropoietin. J. Neurochem. 91, 900-910.

WHO (2006). Neurological disorders: public health challenges. WHO Library Cataloguing-inPublication Data.

Wohlfrom, H., Schinko, J. B., Klingler, M., and Bucher, G. (2006). Maintenance of segment and 
appendage primordia by the Tribolium gene knödel. Mech. Dev. 123, 430-439.

Wrighton, N. C., Farrell, F. X., Chang, R., Kashyap, A. K., Barbone, F. P., Mulcahy, L. S., et al. (1996). Small peptides as potent mimetics of the protein hormone erythropoietin. Science $273,458-$ 463.

Wyder, S., Kriventseva, E. V., Schröder, R., Kadowaki, T., and Zdobnov, E. M. (2007). Quantification of ortholog losses in insects and vertebrates. Genome Biol. 8. doi:10.1186/gb-2007-8-11-r242.

Yamauchi, T., Yoshida, A., and Ueda, T. (2011). Camptothecin induces DNA strand breaks and is cytotoxic in stimulated normal lymphocytes. Oncol. Rep. 25, 347-352.

Yang, C., Xu, Z., Zhao, Z., Li, L., Zhao, T., Peng, D., et al. (2014). A novel proteolysis-resistant cyclic helix B peptide ameliorates kidney ischemia reperfusion injury. Biochim. Biophys. Acta - Mol. Basis Dis. 1842, 2306-2317.

Yang, C., Zhao, T., Lin, M., Zhao, Z., Hu, L., Jia, Y., et al. (2013). Helix B surface peptide administered after insult of ischemia reperfusion improved renal function, structure and apoptosis through beta common receptor/erythropoietin receptor and PI3K/Akt pathway in a murine model. Exp. Biol. Med. 238, 111-119.

Yang, F., Xu, Y.-P., Li, J., Duan, S.-S., Fu, Y.-J., Zhang, Y., et al. (2009). Cloning and characterization of a novel intracellular protein p48.2 that negatively regulates cell cycle progression. Int. J. Biochem. Cell Biol. 41, 2240-2250.

Yarmolinsky, M. B., and De La Haba, G. L. (1959). Inhibition By Puromycin of Amino Acid Incorporation Into Protein. Proc. Natl. Acad. Sci. 45, 1721-1729.

Ye, J., Fang, L., Zheng, H., Zhang, Y., Chen, J., Zhang, Z., et al. (2006). WEGO: A web tool for plotting GO annotations. Nucleic Acids Res. 34, 293-297.

Yoshimura, A., Longmore, G., and Lodish, H. F. (1990). Point mutation in the exoplasmic domain of the erythropoietin receptor resulting in hormone-independent activation and tumorigenicity. Nature 348, 647-49.

Yu, X., Shacka, J. J., Eells, J. B., Suarez-Quian, C., Przygodzki, R. M., Beleslin-Cokic, B., et al. (2002). Erythropoietin receptor signalling is required for normal brain development. Development 129, 505-516.

Zellinger, C., Seeger, N., Hadamitzky, M., Fischborn, S., Russmann, V., Wendt, H., et al. (2011). Impact of the erythropoietin-derived peptide mimetic Epotris on the histopathological consequences of 
status epilepticus. Epilepsy Res. 96, 241-249.

Zhang, H., Fang, X., Huang, D., Luo, Q., Zheng, M., Wang, K., et al. (2017). Erythropoietin signaling increases neurogenesis and oligodendrogenesis of endogenous neural stem cells following spinal cord injury both in vivo and in vitro. Mol. Med. Rep., 264-272.

Zhang, Q., Sakamoto, K., and Wagner, K.-U. (2014a). D-type Cyclins are important downstream effectors of cytokine signaling that regulate the proliferation of normal and neoplastic mammary epithelial cells. Mol. Cell. Endocrinol. 382. doi:10.1016/j.mce.2013.03.016.

Zhang, W., Sun, B., Yu, Z., An, J., Liu, Q., and Ren, T. (2009). High dose erythropoietin promotes functional recovery of rats following facial nerve crush. J. Clin. Neurosci. 16, 554-556.

Zhang, Y., Wang, L., Dey, S., Alnaeeli, M., Suresh, S., Rogers, H., et al. (2014b). Erythropoietin action in stress response, tissue maintenance and metabolism. Int. J. Mol. Sci. 15, 10296-10333.

Zou, Y. R., Zhang, J., Wang, J., Peng, L., Li, G. S., and Wang, L. (2016). Erythropoietin receptor activation protects the kidney from ischemia/reperfusion-induced apoptosis by activating ERK/p53 signal pathway. Transplant. Proc. 48, 217-221. 